Florida International University FIU Digital Commons

FIU Electronic Theses and Dissertations

University Graduate School

$12-4-1995$

\title{
Study on passive and/or active vibration control for flexible structures
}

Qinghua Chen

Florida International University

DOI: $10.25148 /$ etd.FI14060174

Follow this and additional works at: https://digitalcommons.fiu.edu/etd

Part of the Mechanical Engineering Commons

\section{Recommended Citation}

Chen, Qinghua, "Study on passive and/or active vibration control for flexible structures" (1995). FIU Electronic Theses and Dissertations. 2142.

https://digitalcommons.fiu.edu/etd/2142

This work is brought to you for free and open access by the University Graduate School at FIU Digital Commons. It has been accepted for inclusion in FIU Electronic Theses and Dissertations by an authorized administrator of FIU Digital Commons. For more information, please contact dcc@fiu.edu. 


\title{
FLORIDA INTERNATIONAL UNIVERSITY
}

Miami, Florida

\section{STUDY ON PASSIVE AND/OR ACTIVE VIBRATION CONTROL FOR FLEXIBLE STRUCTURES}

A dissertation submitted in partial satisfaction of the requirements for the degree of DOCTOR OF PHILOSOPHY IN MECHANICAL ENGINEERING

\author{
by \\ Qinghua Chen
}

1995 
To: Dean Gordon Hopkins, Ph.D.

College of Engineering and Design

This dissertation, written by Qinghua Chen, and entitled Study on Passive and/or Active Vibration Control for Flexible Structures, having been approved in respect to style and intellectual content, is referred to you for judgement.

We have read this dissertation and recommend that it be approved.

Kuang-Hsi Wu

Gareth J. Knowles

Ton-Lo Wang

Cesar Levy, Major Professor

Date of Defense: December 4, 1995

The dissertation of Qinghua Chen is approved.

Dean Gordon Hopkins

College of Engineering and Design

Dr. Richard L. Campbell

Dean of Graduate Studies

Florida International University, 1995 


\section{ACKNOWLEDGEMENTS}

I wish to thank the members of my committee; Kuang-Hsi Wu, Gareth J. Knowles, Tong-Lo Wang and Cesar Levy, for their helpful comments and patience. I also want to thank Dr. Zhongji Pu for his helpful comment, and Dr. John Koch from SoundCoat Co., INC for his kindness support.

Most of all, I wish to extend my deepest gratitude to my major professor, Dr. Cesar Levy, for his complete support and encouraging comments, and especially for having the confidence in me to give me the chance to do this project. 


\section{ABSTRACT OF THE DISSERTATION \\ STUDY ON PASSIVE AND/OR ACTIVE VIBRATION CONTROL FOR FLEXIBLE STRUCTURES}

by

\section{Qinghua Chen}

Florida International University, 1995

Professor Cesar Levy, Major Professor

The purpose of this research is to present new methods of active and passive vibration control for flexible structures. The study includes: 1) passive viscoelastic damping treatment; 2) active vibration control using layered shape memory alloy (SMA); 3) combined application of viscoelastic damping treatment and SMA; 4) experiments.

In order to maximize damping and save weight of the structure and cost, a partially covered double sandwich cantilever beam model has been presented. It is shown that the double sandwich beam is better than single sandwich beam for some conditions. To take into account of end loads effect of elastic structures such as robot arm or manipulator, a model of partially covered double sandwich cantilever 
beam with mass at free end is given and discussed. Also a more accurate model (Timoshenko model) is discussed. The experiments were done to verify the theoretical results.

The active vibration control by means of layered shape memory alloy actuator is discussed. The layered structure is easy to implement in real application, especially for the existing structures. The control results are compared and discussed. The temperature effects are discussed.

Also a model with combined application of viscoelastic damping treatment and shape memory alloy layer is presented. Both the vibration characteristics and control results are obtained and discussed. The vibration control results for different control schemes are compared and discussed. The temperature effects are also discussed 
1. INTRODUCTION

1.1 The Outline

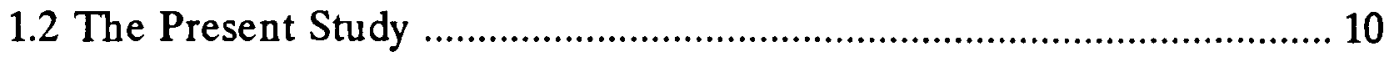

2.1 Vibration Characteristics of Partially Covered Double Sandwich Cantilever Beam

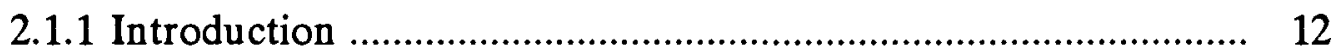

2.1.2 Basic Equation ..................................................................... 13

2.1.3 Frequency and Loss Factor ........................................................19

2.1.4 Comparison of Equation (2-16) with Rao (1978) ...... 26

2.1.5 Numerical Results and Discussion ........................ 27

2.2 Vibration Characteristics of Partially Covered Double Sandwich Cantilever Beam with Concentrated Mass at the Free End ....................... 37

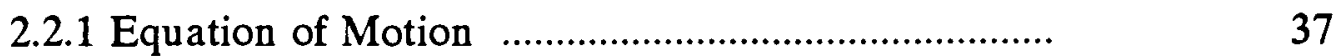

2.2.2 Resonant Frequency and Loss Factor .................................. 43

2.2.3 Numerical Results ............................................. 52

2.3 Vibration Analysis of Partially Covered Double Sandwich Cantilever Timoshenko Beam .................................................................. 62



2.3.2 The Theoretical Model ........................................................ 63

2.3.3 Frequency and Loss Factor ................................................... 70

2.3.4 Comparisons of Numerical Results .............................................73

2.3.5 Conclusions ...................................................................... 84

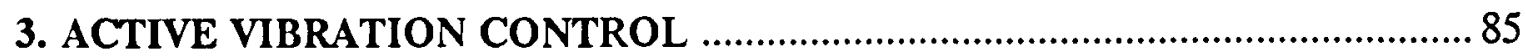

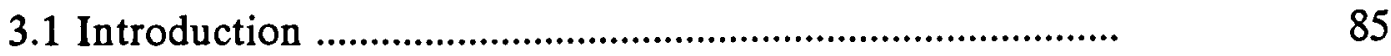

3.2 Formulations of the Problem ...................................................... 87

3.2.1 Governing Equation of the Beam ........................................... 87

3.2.2 Modal Analysis in State Space Variables ................................... 91 
3.3.1 Thermal Bending Moment ................................................... 92

3.3.2 Governing Equation of the Beam ......................................... 94

3.3.3 Modal Analysis in State Space Variables ...................................... 97

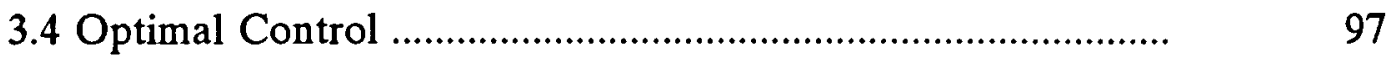



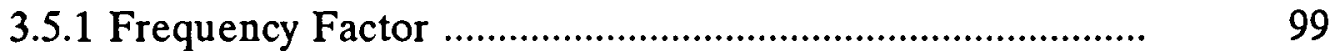

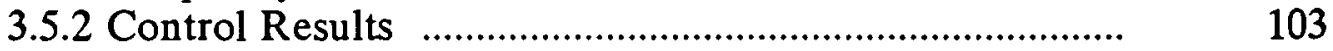

3.6 Conclusion .................................................................

4. COMBINED ACTIVE AND PASSIVE VIBRATION CONTROL .................. 108

4.1 Governing Equation of the Beam ........................................... 108

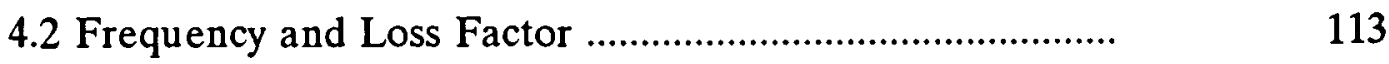

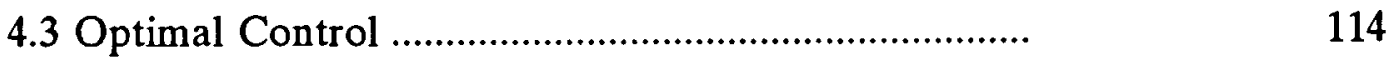



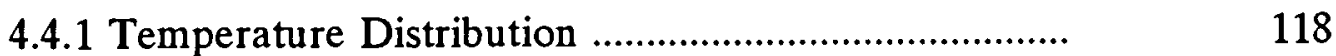

4.4.2 Governing Equations Including Temperature Effect ........ $\quad 124$

4.4.3 Vibration Control ................................................... 125

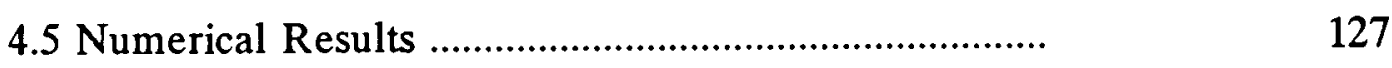

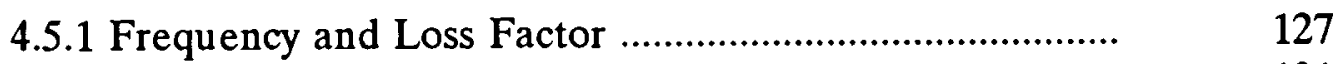

4.5.2 Control Results ...................................................... 131

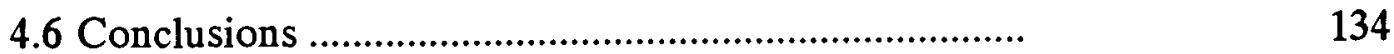

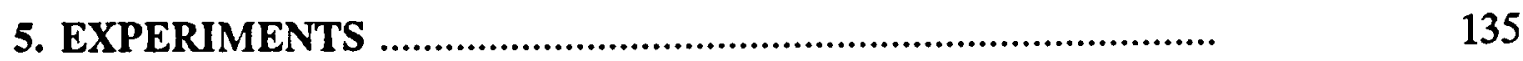

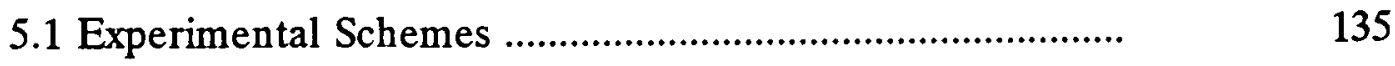

5.2 Vibration Experiments of Constrained Viscoelastic

Damping Structure ................................................................ 
6. CONCLUSION AND FUTURE WORK 


\section{LIST OF FIGURES}

Figure

2.1 The partially covered double cantilever sandwich beam .................... 13

2.2 The geometry and deformation of the beam ................................. 14

2.3 Damping layer coverage length ratio versus frequency factor .............. 28

2.4 Coverage length ratio versus system loss factor .......................... 28

2.5 Damping layer thickness versus system loss factor ....................... $\quad 30$

2.6 Damping layer thickness versus frequency factor ...................... $\quad 30$

2.7 Constraining layer thickness ratio versus system loss factor ............. 32

2.8 Mass density versus frequency factor ................................. 32

2.9 Mass density versus system loss factor ............................... 34

2.10 Young's modulus ratio versus system loss factor ....................... 34

2.11 Damping layer shear modulus ratio versus system loss factor .............. 35

2.12 Comparison between single and double sandwich beam ...................... 35

2.13 Model of the double sandwich cantilever beam with tip mass .............. 38

2.14 Resonant frequency versus mass ratio (the ratio of attached mass to the mass of the beam) ....................... 53

2.15 Frequency ratio versus mass ratio ....................................... 53

2.16 Frequency ratio versus mass ratio ....................................... 56

2.17 Second mode resonant frequency ratio versus mass ratio ................... 56

2.18 System loss factor versus coverage ratio .................................. 58

2.19 Loss factor versus shear modulus ratio .................................. 58 
2.20 Double sandwich beam ...............................................

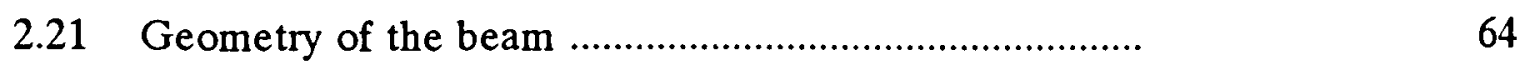

2.22 System loss factor versus damping layer height ratio ................... $\quad 75$

2.23 Frequency ratio versus damping layer height ratio ..................... 75

2.24 System loss factor versus damping layer shear modulus ratio .............. 77

2.25 Frequency factor versus damping layer shear modulus ratio ............... 77

2.26 System loss factor versus constraining layer thickness ratio ............. $\quad 80$

2.27 System loss factor versus coverage length ratio ........................... 80

2.28 System loss factor versus vibration mode .................................

2.29 The effects of Timoshenko factor to frequency factor .................... 82

2.30 The effects of Timoshenko factor to system loss factor ................... 83

3.1 Configuration of beam with SMA layers .................................. 88

3.2 Forces and stresses on beam element .................................. 88

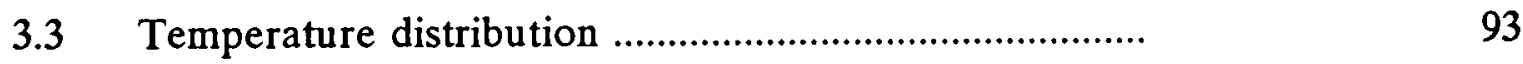

3.4 SMA layer temperature vs. frequency factor ............................. 100

3.5 SMA layer thickness vs. frequency factor ................................ 100

3.6 Beam thickness ratio $h_{2} / L$ vs. frequency factor ........................ 102

3.7 SMA Young's modulus ratio vs. frequency factor ............................ 102

3.8 Comparison of results with (solid line)

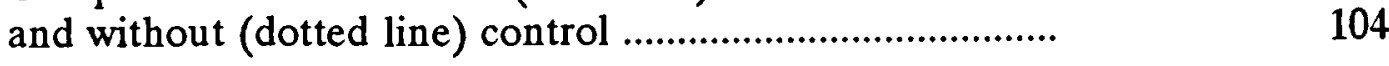

3.9 Control results for different SMA materials .............................. 104

3.10 Control results for different SMA Young's modulus ratio ................. 105

3.11 Vibration control time vs. SMA Young's modulus ratio ..................... 105 
4.1 Beam with SMA and viscoelastic damping treatments ...................... 109

4.2 Forces and stresses on beam element ..................................... 109

4.3 Temperature distribution for beam with SMA and viscoelastic layer ......119

4.4 SMA layer temperature vs. frequency factor ............................. 128

4.5 SMA layer temperature vs. system loss factor ............................ 128

4.6 Shear modulus ratio of viscoelastic layer vs. frequency factor and system loss factor

4.7 Damping layer height ratio vs. frequency factor

and system loss factor

4.8 Comparison of results with (dotted line) and without (solid line) active vibration control

4.9 Vibration response for different damping material loss factor ........... 132

4.10 Active vibration control results for different damping material loss factor

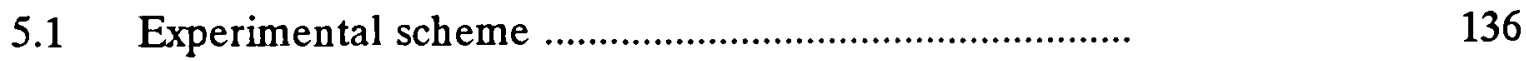

5.2 Attachment of damping layer and constraining layer ...................... 137

5.3 Coverage length ratio vs. system loss factor .............................. 139

5.4 Coverage length ratio vs. natural frequency of the system ............... 139

5.5 The ratio of end mass to mass of beam vs. system loss factor ............. 141

5.6 The ratio of end mass to mass of beam vs. natural frequency ............. 141

5.7 System loss factor comparison of single sandwich with double sandwich beam 


\section{LIST OF SYMBOLS}

$\mathrm{A}_{\mathrm{i}}$

$A_{c 1}, A_{c 2} \quad$ cross-sectional area of the damping layers

$A_{s i}$

$\mathrm{A}_{\mathrm{fi}}$

D

$\mathrm{D}_{\mathbf{1 1}}$

$\mathrm{E}_{\mathrm{i}}$

$E_{1}$

$\mathrm{E}_{\mathrm{Aj}}$

$\mathrm{E}_{\mathrm{Mi}}$

$\mathrm{G}_{\mathrm{i}}$

$G_{i}$

$\mathrm{G}_{\mathrm{ci}}^{*}$

$\mathrm{h}_{\mathrm{i}}$

$\mathrm{h}_{\mathrm{cl}}, \mathrm{h}_{\mathrm{c} 2}$

$\mathrm{h}_{01}$

$\mathrm{h}_{02}$

$\mathrm{H}_{\mathrm{i}}$

j

$\mathrm{k}_{\mathrm{n}}^{*}$

$k_{n}$ cross-sectional area of layer $i$

start temperature of austenite transformation

end temperature of austenite transformation

$=\left[E_{1} h_{1}{ }^{3}+E_{3} h_{3}{ }^{3}+E_{4} h_{4}{ }^{3}\right] / 12$

$=E_{1} h_{1}{ }^{3} / 12$ bending rigidity of the original beam

Young's modulus of the $i$ th layer

dimensionless Young's modulus of $i$ th layer

the austenite Young's modulus of SMA layer

the martesite Young's modulus of SMA layer

shear modulus of the $i$ th layer

dimensionless shear modulus of $i$ th layer

complex shear modulus of the $i$ th layer $=G_{\mathrm{ci}}\left(1+\mathrm{j} \eta_{c i}\right)$

thickness of the $i$ th layer

thickness of the damping layers

$=\mathrm{h}_{\mathrm{c} 2}+\left(\mathrm{h}_{1}+\mathrm{h}_{4}\right) / 2$

$=\mathrm{h}_{\mathrm{c} 1}+\mathrm{h}_{\mathrm{c} 2}+\mathrm{h}_{1}+\left(\mathrm{h}_{3}+\mathrm{h}_{4}\right) / 2$

dimensionless thickness of $i$ th layer

$=\sqrt{ }-1$

complex characteristic value

real characteristic value 
constrained damping treatment length ratio

L length of the beam

$\mathrm{L}_{1} \quad$ length of constrained damping treatment coverage

$M_{1}, M_{2} \quad$ bending moment at $x=0$ and $x=L$, respectively

$M_{0} \quad$ attached mass at the free end

$M_{\mathfrak{i l}} \quad$ temperature at the end of martensite transformation

$\mathrm{M}_{\mathrm{si}} \quad$ temperature at the beginning of martensite transformation

$\mathrm{M}_{\mathrm{T}} \quad$ bending moment

$\mathrm{m}_{\mathrm{i}} \quad$ mass per unit length of $i$ th layer

m mass per unit length of section 1 (constrained part)

$\mathrm{p} \quad$ real frequency factor

$\mathrm{p}^{*} \quad$ complex frequency factor

$\mathrm{P}_{\mathrm{i}} \quad$ axial force of the $i$ th layer

$\mathrm{S}_{1}, \mathrm{~S}_{2} \quad$ shear force

t time

$\mathfrak{\tau}$ dimensionless time

$T_{i} \quad$ time dependent function, temperature distribution

$\mathbf{u}_{\mathrm{i}} \quad$ axial displacement of the $i$ th layer

$\overline{\mathrm{u}}_{\mathrm{i}} \quad$ dimensionless axial displacement

$\overline{\mathrm{u}}_{\mathrm{in}} \quad$ the spatial part of the solution

$w_{1}, w_{2} \quad$ transverse displacement of section 1 and 2 , respectively

$\overline{\mathbf{w}}$

dimensionless transverse displacement 


$\begin{array}{ll}\overline{\mathrm{w}}_{\mathrm{n}} & \text { the spatial part of the solution } \\ \mathrm{x} & \text { axial or longitudinal coordinate } \\ \overline{\mathrm{x}} & \text { dimensionless axial coordinate } \\ \mathrm{X}_{\mathrm{c}} & \text { state space variable vector } \\ \alpha_{\mathrm{i}} & \text { the thermal coefficient of the } i \text { th layer } \\ \alpha_{\mathrm{Ti}} & \text { the thermal diffusivity of the } i \text { th layer } \\ \delta(\mathrm{x}) & \text { Dirac delta function } \\ \delta_{\mathrm{ij}} & \text { Kronecker delta } \\ \gamma_{\mathrm{i}} & \text { shear at the neutral axis of the } i \text { th layer } \\ \xi_{\mathrm{i}} & \text { martensite fraction of SMA layer, i.e. } \% \text { martensite } \\ \rho_{\mathrm{i}} & \text { stress of the } i \text { th layer } \\ \rho_{\mathrm{ri}} & \text { recovery stress of SMA layer } \\ \tau_{\mathrm{i}} & \text { shear stress of the } i \text { th layer } \\ \Phi_{\mathrm{i}} & \text { eigenfunction of the beam } \\ \psi_{\mathrm{i}} & \text { slope of the deflection curve of } i \text { th layer due to bending }\end{array}$




\section{Chapter 1 Introduction}

\subsection{The Outline}

Recent developments in industry require more and more applications of flexural structures ( e.g., flexural robot arms and large space manipulators). Due to movement of heavy payloads and the high moving speeds encountered, undesired low frequency vibrations and instability may occur. For example, because of the flexibility, operations are sometimes very difficult or delayed while operators wait for the oscillations to die out. For remote manipulator system(RMS), a recent study (Newsom et al., 1990) showed that the initial phases of space station assembly would require about 47 hours of RMS operation time, but about $20-30 \%$ of the time would be waiting for the vibrations of the RMS to die out. Also the flexibility would make the structural response a significant contributor to space vehicle dynamics (Scott $e t$ al., 1993). Hence an effective vibration control scheme by which the size and weight of the structure will not increase greatly is strongly required. On the other hand, machines nowadays need to be stronger, lightweight and capable of a very high speed operation. The high speeds of operation and lightweight designs of structure will result in larger amplitude vibration of flexible structure during operation which leads to a reduction in the accuracy and precision of machine operations. This requires the introduction of additional damping (Tzou and Wan, 1990; Reinhorn and Manolis, 1985).

The vibration problem of a beam carrying a concentrated mass or 
concentrated masses and having arbitrary boundary conditions at the end supports is of great interest to the practical engineer. For example, this system may be considered as a model of a robot arm with a mass in its end effector. The vibrational aspects of this kind of system may be of great interest, when, due to some motion at the shoulder joint, the system is made to oscillate. Rao (1990) discussed the cantilever Euler beam with concentrated tip mass as one of the examples in his book. Pan (1965) studied the transverse vibration of an Euler beam carrying a system of heavy bodies. Rami Reddy and Amba-Rao (1973) solved the problem of vibrations of beams with non-classical boundary conditions (rotational and translational springs at the supports) using transfer matrices. Amba-Rao and Hussaini (1975) obtained a closed-form solution for the classical problem of a beam. The dynamic properties of structures carrying concentrated masses were studied by Laura et al. $(1977 ; 1987)$. Stokey and Zorowski (1959) solved the normal vibrations of a uniform plate carrying any number of finite masses. An approximate method to find the fundamental frequency of a restrained cantilever beam carrying a tip heavy body was presented by Gurgoze (1986). Although the vibration properties of such system were studied, the control of vibration needs to be done.

Vibration control techniques are generally classified into two major categories: passive vibration control and active vibration control (actuator/controller). The passive method includes proper design of system to avoid the resonance and using damping treatment (viscous damper, viscoelastic damping, etc ) which dissipates the energy of the vibrating structures. Unconstrained and constrained viscoelastic layer 
damping treatments were first analyzed and developed in the late 1950's. Kerwin (1959) presented the first analysis of the problem followed by Ditaranto (1965), Rao (1978), and Mead (1982). The response of a damped sandwich beam to random excitation was discussed by Xi et al (1986). Chen and Xi (1987) obtained the improved differential equations for the damped sandwich-type beam. That analysis was continued by Levy and Chen (1994a) who investigated the double sandwich type beam, which may be used as a model for robot arms and space structures. Also a concentrated mass attached at the free end of such a structure was studied (Chen and Levy, 1994a). The improved equation for the double sandwich type beam was also obtained by Levy and Chen (1994b). Other studies of passive damping technology were undertaken by Nashi (1992), and, Levraea and Rogers (1992). Alberts, et al (1992) studied the effectiveness of viscoelastic passive damping augmentation for the space shuttle remote manipulator system. The application of viscoelastic damping in advanced composite large space structures was studied by Edberg and Bicos (1991). The design guidelines for favorable trade-offs between damping benefits and the associate stiffness, strength and weight penalties was studied by Pruce et al. (1989). Passive vibration controls of flexible robot manipulators using viscoelastic damping materials were also studied (Alberts et al, 1986; Tzou, 1988). This method was found to reduce the resonant peaks through material damping and was suitable for vibration control of flexural structures. Nonlinear vibration analysis with viscoelastic damping treatment was performed by Wojciech et al. (1990), Lukasiewich and Xia (1993), and Ganeriwala (1994). 
Because of the weight consideration of the structure and the cost of materials involved in fully covering the beam with viscoelastic material, some compromise in its design is necessary. One way to offset the increase in mass is to use partial coverage of the primary layer. Models of the effects of partial coverage on a beam and plate have been presented by Lall et al. (1987; 1988). Garrison et al. (1992) studied the effect of partially covered plates with viscoelastic material. Chen (1986) discussed the eigenvalue and loss factor of a partially covered sandwich-type beam. In order to increase the damping effect, fully covered multi-layer damped structures have been studied by many authors (Chen and Levy, 1994a; Levy and Chen, 1994a,1994b;Agbrasiere and Groutenhuis, 1968; Asnani and Nakra, 1970; He and Rao, 1990). Symmetrical multi-layer beams with viscoelastic damping were studied by Agbasiere and Groutenhuis (1968). Asnani and Nakra (1970) investigated nonsymmetrical beams. He and Rao (1992) discussed multiple viscoelastic layer damped beams. Optimal length of coverage by constrained viscoelastic layer damping was investigated by Plunkett and Lee (1970), and Mantena (1991). The optimal location and shape of viscoelastic coating coverage giving the best damping performance for a given weight of material was investigated by Cheng and Lapointe (1994). However, a drawback to this method of vibration control is that the increase in damping can only be optimized for specific narrow ranges of temperature and frequency. Also after the completion of design, one can not change the system parameters.

In recent years, improvements in materials and manufacturing process, and more specifically, advances in data acquisition and computer technologies such as 
MATLAB control toolbox have made it possible to apply sophisticated active control techniques to vibration structures (Browning and Medaugh, 1992). Various vibration control methods have been suggested (Browning and Medaugh, 1992; Bailey and Hubbard, 1985; Bar-kanal and Kaufman, 1984; Tzou, 1989; Sakawa et al., 1985; Matsuno and Sakawa, 1990). Knowles (1990) presented point sensing and control systems. Active damping for vibration control of a flexible structure was also studied (Holnicki-Szulc, 1993; Scott, 1993; Habib, 1991). For flexible structures, although traditional control methods do stabilize resonance at specific frequencies, problems arise when the structure has modes at closely spaced frequencies (Gaudreault et al, 1992). It is better to use distributed control, i.e., using smart materials distributed over the structure (Duclos et al., 1989). The most prominent actuation techniques that were examined in recent investigations have been based on the properties of piezoelectric materials, shape memory alloys, and magnetostrictive devices (Tzou and Anderson,1992). For the flexible vibration control, a flexible piezoelectric material is required to be closely coupled with the robot or machine components (Tzou,1989). Polyvinylidene fluoride (PVDF) is found to be very attractive for distributed sensing and control applications because of its very good properties (Wojciech et al., 1990; Lukasiewich and Xia, 1993). The application of active control with piezoelectric ceramics were also found (Crawley and Luis, 1987; Fanson and Garba, 1988). Because the piezoelectric ceramics are rigid and brittle, there are some limitations for vibration control of flexible structures even though they display high force-strain characteristics. Also, the control for the method using 
piezoelectric materials may be significantly distorted at low frequency due to the limitation of the material itself (Tzou, 1989) which is very important for robot arms or space manipulators. There are also limitations to the magnitudes of the electric fields that can be applied to piezoelectric materials (Tzou and Anderson, 1992). Most recently, the investigations of the applications of magnetostrictive materials to vibration control have been reported (Fujita et al., 1994; Flatau et al., 1993; Bryant et al., 1993; Apollonov et al., 1992). Magnetostrictive materials undergo a deformation when an electric field is applied to them. If we could solve the difficulties of applying the necessary magnetic field to the surface of the actuator, this is very useful material.

The applications of shape memory alloy (SMA) in the fields of structural acoustic control and vibration control have attracted more and more attention since the late 1980's (Rogers, 1990; Saunders et al., 1990; Chen and Levy, 1994). Buehler and Wiley (1965) presented the first analysis of Shape Memory Alloys (SMA). The properties of different kinds of SMA have been studied by Wu et al. $(1993,1994)$ and by $\mathrm{Pu}$ and $\mathrm{Wu}$. The composites with SMA have the ability to change material properties, induce large internal forces in the materials, modify the stress and strain state of the structure and alter the body's configuration (Rogers et al., 1989). These charateristics are very useful for vibration control. The applications of SMA composites for vibration and structural acoustic control were presented (Rogers, 1990; Saunders et al., 1990; Rogers et al., 1991). It was shown that, for the SMA hybrid beam with a nitinol volume of $15 \%$, the first natural frequency of the SMA 
hybrid beam increased from $21 \mathrm{~Hz}$ at room temperature to $62 \mathrm{~Hz}$ when the actuators were heated to $300^{\circ} \mathrm{F}\left(149^{\circ} \mathrm{C}\right)$ (Rogers et al., 1991; Barker, 1989). The application of SMA actuators to robot systems was evaluated by many authors (Katsutoshi, 1993; Lashlee et al., 1993; Fukuda et al., 1990a, 1990b). Micro size thin film actuators were studied by Katsutoshi (1993). Fukuda et al. (1990a, 1990b) studied a distributed type SMA actuators and presented an application of such actuators to underwater mobile robotic mechanism. For the ordinary control method (pulse width modulation control method), the maximum voltage supplied to the SMA actuator must be kept low in order to prevent the SMA from " burning " because its temperature is unknown. This would make the response of an SMA actuator very slow. Typical SMA such as Nitinol display low frequency characteristics. But by varying the mix and manufacturing process, higher bandwidths can be obtained. However, these are not generally available commercially. Kuribayashi (1991) proposed a control method to improve the response speed of the SMA actuator by limiting the maximum temperature of the SMA to below the limiting value instead of by limiting the maximum voltage supplied to the SMA.

But there is much to be learned, e.g., the influence of the residual stress and high temperatures on the extent, duration, and repeatability of the Shape Memory Effect (SME) and dynamic actuator and sensing characteristics (Rogers, 1990), and the dynamic models. The most difficult problem is that the embedded fiber structures (Rogers, 1990; Saunders et al, 1990; Rogers et al, 1991; Barker, 1989) are very hard to implement in practical applications. For high frequency vibration control, there 
would be difficulties because of the time needed for SMA fibers to cool down after heating up and also the problem of how to embed the fiber in real applications needs to be solved.

In addition, pure active vibration control has some potential difficulties. For example, control spillover and/or observation spillover have the potential to degrade the performance and even lead to instability (Alberts et al., 1992; Balas, 1978). For the uncompensated vibration modes, there is a need to improve the robustness of the system to model uncertainties. In addition, the practical application in real vibration control systems is still unclear. This uncertainty is especially true for the control of large structure motion and vibration (Duclos et al., 1989),i.e., there is a great difference between control of a simple beam or plate and control of real complex structure.

Most recently, the idea of combined research of passive and active vibration control has been presented (Simonian et al., 1984). Koshigeo (1993) studied the active and passive damping for a plate with piezoelectric transducers. Gaudreault and coworkers presented the simultaneous design of active vibration control (weighting the passive damping forces like the active control forces) and passive viscous (Gaudreault et al., 1992) and viscoelastic damping (Gaudreault et al., 1993). The simultaneous design of viscoelastic damping (Gaudreault, 1993) is that for the viscoelastic dampers on some points of the structure, the performance index was minimized by employing the passive damping forces as weighting functions. The experimental results involving control of a simple flexible link have illustrated the 
effectiveness of combined active and passive control strategies (Alberts et al., 1990; 1992). The controllability of vibration beams with embedded smart composite was studied by Arockiasamy (1992). In this case, the smart material has elastic/viscoelastic properties. Agnes and Napolitano (1993) investigated active constrained layer viscoelastic damping. It is shown that the modal damping increases significantly for the fundamental mode of a simply supported beam. Also the effectiveness of the treatment is good over a broader temperature range as well. The simultaneous application of constrained viscoelastic damping and layered shape memory alloy was first presented by Chen and Levy (1994b). The shape memory alloy layer is used as actuator with viscoelastic damping 1.) as supplementary control for the unsuppressed modes, and, 2.) as method of controlling instability. Also the model presented is a layered structure, which is easy to implement compared with the previous structures (Rogers, 1990; Saunders et al., 1990; Rogers et al., 1991; Barker, 1989).

As stated before, there is still much work to be done for the successful vibration control of flexible structures. For combined application of viscoelastic damping treatment and SMA to vibration control, the research is only at the beginning stage. 


\subsection{The Present Study}

As stated before, very limited research is about the vibration of double sandwich cantilever beam. Also there is no report about the combined application of SMA actuators and constrained viscoelastic damping treatment.

This study includes 1) passive viscoelastic damping treatment for vibration control of cantilever beam; 2) active vibration control using layered shape memory alloy; 3) combined application of viscoelastic damping treatment and shape memory alloy; 4) experiments.

In order to maximize damping and save weight of the structure and cost, a partially covered double sandwich cantilever beam model has been presented. It is shown that the double sandwich beam is better than a single sandwich beam for some conditions. To take into account of end loads effect of robot arm or manipulator, a model of partially covered double sandwich cantilever beam with mass at free end is given and discussed. Also a more accurate model (Timoshenko model) is discussed.

The active vibration control by means of layered shape memory alloy actuator is discussed. The layered structure is easy to implement in real application, especially for the existing structures. A linear control law is determined based on optimal regulator theory. The control results are compared and discussed.

For the pure passive vibration control, we can not change the system parameters after the completion of design. Hence a model with combined application of viscoelastic damping treatment and shape memory alloy is presented. Both the 
mathematical and experimental models are established. The vibration characteristics of such a combined system are analyzed. It is shown that change of the elastic modulus of the SMA layer will affect the system loss factor and natural frequency; Also the recovery stress of the SMA layer functions like an excitation, adjusting the excitation. A linear control law is determined based on the digital optimal regulator theory. The vibration control results are obtained and discussed.

Since the structure may be used in the temperature variation enviroment or because of the application of SMA layers in the structure, the temperature effects to the system frequency and loss factor are analyzed.

Experiments are performed to analyze the dynamic characteristics of the system. The experimental scheme is mainly composed of the excitation system, flexible beam structure and data acquisition system. In the test, the effects of damping coverage length, location, etc., are analyzed. 


\section{Chapter 2 Passive Damping Treatment}

2.1 Vibration characteristics of partially covered double sand wich cantilever beam

\subsubsection{Introduction}

Numerous papers have been published since the 1950's on the vibration of three-layered sandwich beams and plates (Kerwin, 1959; Ditaranto, 1965; Rao, 1978; Mead, 1882; etc). In this section, the vibration of a cantilever beam with partially covered double sandwiched visco-elastic materials has been studied. The differential equations governing the vibration of the beam are derived, and the corresponding boundary conditions are given. This model may be used for the study of robot arm vibration. Also, in the derivation of the boundary conditions, the continuity conditions between the damped part and the primary (undamped) part are used. Comparison between the present equations and other equations found in the literature have been made. The frequency and loss factor of the system are analyzed. The effects of different parameters (including physical and geometrical parameters, mass density, mode, and core loss factor) on resonance frequency and system loss factor are also discussed. Also, the double sandwich type system is compared to a single sandwich type system to determine if it has superior damping capabilities. The equations obtained for numerical calculation are nonlinear, complex valued equations. The solution of these equations was obtained by the computer. 


\subsubsection{Basic Equation}

The double sandwiched cantilever beam is shown in Figure 2.1. The beam is partly damped by viscoelastic material. There are two damping layers and two constraining layers. The following assumptions are made in the analysis:

1) the beam deflection is small and uniform across any section.

2) the primary beam and the upper and lower constraining beams are assumed to be isotropic.

3) The longitudinal and rotatory inertia effects of the beam are neglected.

4) The damping layers carrying shear but no direct stress, are assumed to be linear viscoelastic.

5) No slip occurs at the interface between the layers.

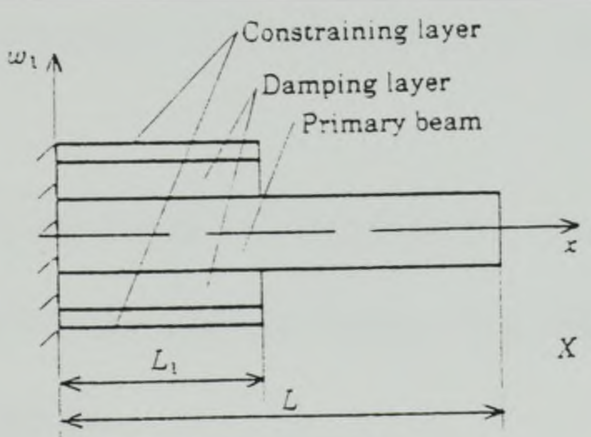

(a)

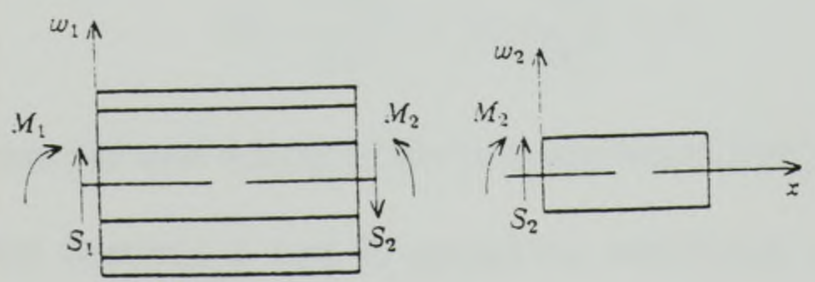

(b)

Figure 2.1 The partially covered double cantilever sandwich beam: (a) the full beam; (b) the two sections with loads and moments. 


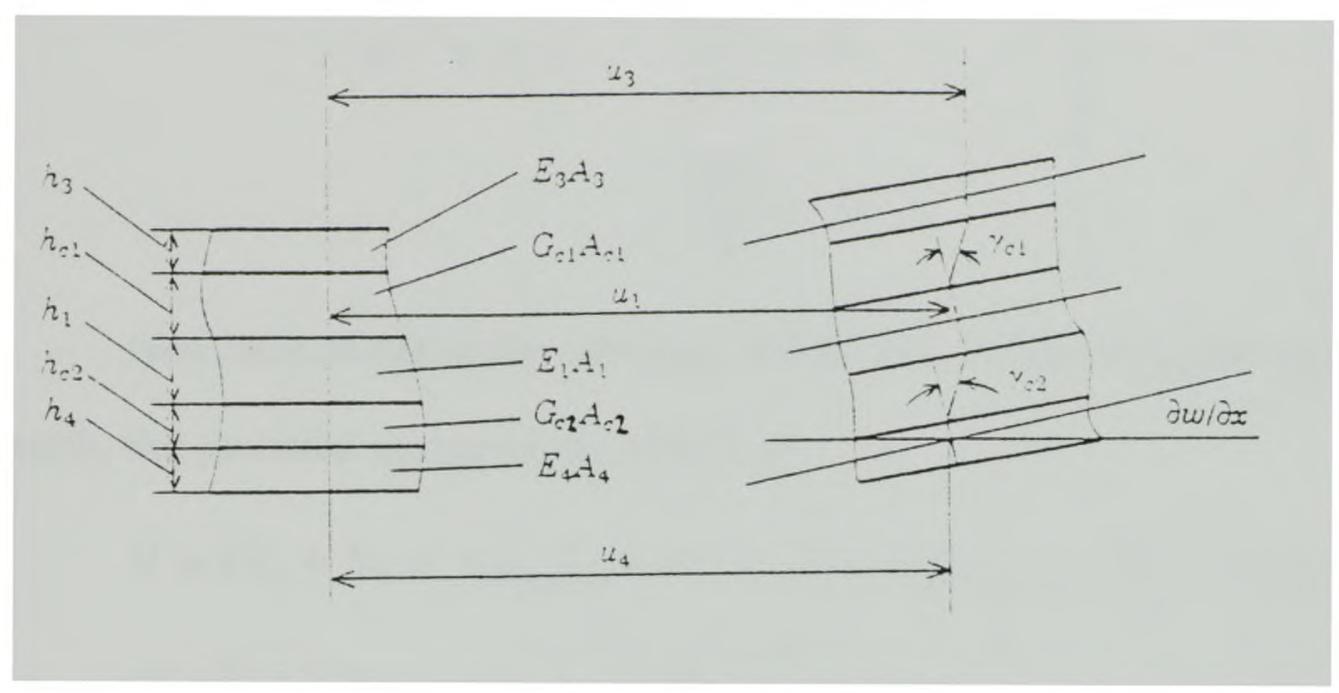

Figure 2.2 The geometry and deformation of the beam

The beam can be separated into two sections. Section 1 is a double sandwichtype beam, and Section 2 is the ordinary beam. The differential equation of motion for Section 2 is governed by:

$$
D_{t 1} \frac{\partial^{4} w_{2}}{\partial x^{4}}+m_{2} \frac{\partial^{2} w_{2}}{\partial t^{2}}=0
$$

where $\mathrm{m}_{2}$ the mass per unit length of the primary beam and $D_{t 1}=E_{1} h_{1}^{3} / 12$. For the interface between Sections 1 and 2, geometric continuity and generalized force continuity must apply. This will allow us to obtain the boundary conditions. 


$$
\left.\begin{array}{ll}
\text { at } \quad \mathrm{x}=\mathrm{L}_{1} \quad \mathrm{D}_{\mathrm{t} 1} \frac{\partial^{2} \mathrm{w}_{2}}{\partial \mathrm{x}^{2}}-\mathrm{M}_{2}=0 \\
\mathrm{D}_{\mathrm{t} 1} \frac{\partial^{3} \mathrm{w}_{2}}{\partial \mathrm{x}^{3}}+\mathrm{S}_{2}=0
\end{array}\right\}
$$

Now let us consider the vibration of Section 1. If the composite part has unit width, the potential energy of the system is:

$$
\begin{aligned}
& V=\left(V_{1}+V_{3}+V_{4}\right)_{\text {bending }}+\left(V_{1}+V_{3}+V_{4}\right)_{\text {extension }}+\left(V_{c 1}+V_{c 2}\right)_{\text {shearing }} \\
& \left(V_{1}+V_{3}+V_{4}\right)_{\text {bending }}=\frac{1}{2} \int_{0}^{L_{1}} D\left(\frac{\partial^{2} w_{1}}{\partial x^{2}}\right)^{2} d x \\
& \left(V_{1}+V_{3}+V_{4}\right)_{\text {bending }}= \\
& \frac{1}{2} \int_{0}^{L_{1}}\left[E_{1} A_{1}\left(\frac{\partial u_{1}}{\partial x}\right)^{2}+E_{3} A_{3}\left(\frac{\partial u_{3}}{\partial x}\right)^{2}+E_{4} A_{4}\left(\frac{\partial u_{4}}{\partial x}\right)^{2}\right] d x \\
& \left(V_{c 1}+V_{c 2}\right)_{\text {shearing }}=\frac{1}{2} \int_{0}^{L_{1}}\left(G_{c 1} A_{c 1} \gamma_{c 1}^{2}+G_{c 2} A_{c 2} \gamma_{c 2}^{2}\right) d x
\end{aligned}
$$

where $D=\left(E_{1} h_{1}{ }^{3}+E_{3} h_{3}{ }^{3}+E_{4} h_{4}{ }^{3}\right) / 12$. The total kinetic energy of the composite beam after neglecting longitudinal and rotatory inertia is: 


$$
\mathrm{T}=\frac{1}{2} \int_{0}^{\mathrm{L}_{\mathrm{L}}} \mathrm{m}\left(\frac{\partial \mathrm{w}_{1}}{\partial \mathrm{t}}\right)^{2} \mathrm{dx}
$$

From Figure 2.2, we have the following relationships:

$$
\begin{aligned}
& \mathrm{u}_{1}-\mathrm{u}_{4}=\left[\mathrm{h}_{\mathrm{c} 2}+\frac{1}{2}\left(\mathrm{~h}_{1}+\mathrm{h}_{4}\right)\right] \frac{\partial \mathrm{w}_{1}}{\partial \mathrm{x}}-\mathrm{h}_{\mathrm{c} 2} \gamma_{\mathrm{c} 2}, \\
& \mathrm{u}_{3}-\mathrm{u}_{1}=\left[\mathrm{h}_{\mathrm{c} 1}+\frac{1}{2}\left(\mathrm{~h}_{1}+\mathrm{h}_{3}\right)\right] \frac{\partial \mathrm{w}_{1}}{\partial \mathrm{x}}-\mathrm{h}_{\mathrm{c1}} \gamma_{\mathrm{c} 1} .
\end{aligned}
$$

The fourth assumption implies that the sum of forces in the longitudinal direction is zero, i.e.,

$$
\mathrm{E}_{1} \mathrm{~A}_{1} \frac{\partial \mathrm{u}_{1}}{\partial \mathrm{x}}+\mathrm{E}_{3} \mathrm{~A}_{3} \frac{\partial \mathrm{u}_{3}}{\partial \mathrm{x}}+\mathrm{E}_{4} \mathrm{~A}_{4} \frac{\partial \mathrm{u}_{4}}{\partial \mathrm{x}}=0
$$

From Eqs. (2-8), (2-9), and (2-10), we obtain:

$$
\begin{gathered}
\frac{\partial \mathrm{u}_{1}}{\partial \mathrm{x}}=\left(\mathrm{B}_{1} \mathrm{~h}_{01}-\mathrm{B}_{2} \mathrm{~h}_{02}\right) \frac{\partial^{2} \mathrm{w}_{1}}{\partial \mathrm{x}^{2}}+\mathrm{B}_{2} \mathrm{~h}_{\mathrm{c} 1} \frac{\partial \gamma_{\mathrm{c} 1}}{\partial \mathrm{x}}-\mathrm{B}_{3} \mathrm{~h}_{\mathrm{c} 2} \frac{\partial \gamma_{\mathrm{c} 2}}{\partial \mathrm{x}} \\
\frac{\partial \mathrm{u}_{3}}{\partial \mathrm{x}}=\mathrm{B}_{4} \frac{\partial^{2} \mathrm{w}_{1}}{\partial \mathrm{x}^{2}}-\mathrm{B}_{8} \mathrm{~h}_{\mathrm{c} 1} \frac{\partial \gamma_{\mathrm{cl}}}{\partial \mathrm{x}}-\mathrm{B}_{3} \mathrm{~h}_{\mathrm{c} 2} \frac{\partial \gamma_{\mathrm{c} 2}}{\partial \mathrm{x}} \\
\frac{\partial \mathrm{u}_{4}}{\partial \mathrm{x}}=\mathrm{B}_{5} \frac{\partial^{2} \mathrm{w}_{1}}{\partial \mathrm{x}^{2}}+\mathrm{B}_{2} \mathrm{~h}_{\mathrm{c} 1} \frac{\partial \gamma_{\mathrm{c} 1}}{\partial \mathrm{x}}+\mathrm{B}_{6} \mathrm{~h}_{\mathrm{c} 2} \frac{\partial \gamma_{\mathrm{c} 2}}{\partial \mathrm{x}},
\end{gathered}
$$

where the parameters defined in (2-11)-(2-13) are given as follows:

$$
\begin{aligned}
& \mathrm{h}_{01}=\mathrm{h}_{\mathrm{c} 2}+\left(\mathrm{h}_{1}+\mathrm{h}_{4}\right) / 2 \\
& \mathrm{~h}_{02}=\mathrm{h}_{\mathrm{c} 1}+\mathrm{h}_{\mathrm{c} 2}+\mathrm{h}_{1}+\left(\mathrm{h}_{3}+\mathrm{h}_{4}\right) / 2 \\
& \mathrm{~B}_{1}=\left(\mathrm{E}_{3} \mathrm{~A}_{3}+\mathrm{E}_{4} \mathrm{~A}_{4}\right) / \mathrm{EA} \\
& \mathrm{B}_{2}=\mathrm{E}_{3} \mathrm{~A}_{3} / \mathrm{EA}
\end{aligned}
$$




$$
\begin{aligned}
& \mathrm{B}_{3}=\mathrm{E}_{4} \mathrm{~A}_{4} / E A \\
& \left.\mathrm{~B}_{4}=\left[\left(\mathrm{E}_{1} \mathrm{~A}_{1}+\mathrm{E}_{4} \mathrm{~A}_{4}\right) \mathrm{h}_{02}-\mathrm{E}_{1} \mathrm{~A}_{1} \mathrm{~h}_{01}\right)\right] / \mathrm{EA} \\
& \mathrm{B}_{5}=-\left(\mathrm{E}_{1} \mathrm{~A}_{1} \mathrm{~h}_{01}+\mathrm{E}_{3} \mathrm{~A}_{3} \mathrm{~h}_{02}\right) / E A \\
& \mathrm{~B}_{6}=\left(\mathrm{E}_{1} \mathrm{~A}_{1}+\mathrm{E}_{3} \mathrm{~A}_{3}\right) / E A \\
& \mathrm{~B}_{7}=\mathrm{B}_{1} \mathrm{~h}_{01}-\mathrm{B}_{2} \mathrm{~h}_{02} \\
& \mathrm{~B}_{8}=\left(\mathrm{E}_{1} \mathrm{~A}_{1}+\mathrm{E}_{4} \mathrm{~A}_{4}\right) / E A \\
& \mathrm{EA}=\mathrm{E}_{1} \mathrm{~A}_{1}+\mathrm{E}_{3} \mathrm{~A}_{3}+\mathrm{E}_{4} \mathrm{~A}_{4} .
\end{aligned}
$$

Work done by external forces is given by:

$$
W=\left.\left(-M_{1} \frac{\partial w_{1}}{\partial x}+S_{1} w_{1}\right)\right|_{x=0}+\left.\left(M_{2} \frac{\partial w_{1}}{\partial x}-S_{2} w_{1}\right)\right|_{x=L_{1}}
$$

where $M_{1}, M_{2}$ and $S_{1}, S_{2}$ are the moments and shear forces, respectively, and the subscript 1,2 indicates the $x=0$ side and $x=L_{1}$ side, respectively. We next apply Hamilton's principle:

$$
\delta \int_{4}^{t_{2}}(T-V+W) d t=0
$$

We obtain the differential equations of motion of the double sandwiched beam in a matrix form:

$$
\left[\begin{array}{lll}
a_{11} & a_{12} & a_{13} \\
a_{21} & a_{22} & a_{23} \\
a_{31} & a_{32} & a_{33}
\end{array}\right]\left\{\begin{array}{l}
w_{1} \\
\gamma_{c 1} \\
\gamma_{c 2}
\end{array}\right\}=\left\{\begin{array}{l}
0 \\
0 \\
0
\end{array}\right\}
$$

where the $a_{i j}$ 's are defined as follows: 


$$
\begin{aligned}
& a_{11}=m \frac{\partial^{2}}{\partial t^{2}}+\left(D+E_{1} A_{1} B_{7}^{2}+E_{3} A_{3} B_{4}^{2}+E_{4} A_{4} B_{5}^{2}\right) \frac{\partial^{4}}{\partial x^{4}} \\
& a_{12}=\left(E_{1} A_{1} B_{2} B_{7}-E_{3} A_{3} B_{4} B_{8}+E_{4} A_{4} B_{2} B_{5}\right) h_{c 1} \frac{\partial^{3}}{\partial x^{3}} \\
& a_{13}=-\left(E_{1} A_{1} B_{3} B_{7}-E_{3} A_{3} B_{3} B_{4}+E_{4} A_{4} B_{5} B_{6}\right) h_{c 2} \frac{\partial^{3}}{\partial x^{3}} \\
& a_{21}=\left(E_{1} A_{1} B_{2} B_{7}-E_{3} A_{3} B_{4} B_{8}+E_{4} A_{4} B_{2} B_{5}\right) h_{c 1} \frac{\partial^{3}}{\partial x^{3}} \\
& a_{22}=\left(B_{2}^{2} E_{1} A_{1}+B_{8}^{2} E_{3} A_{3}+B_{2}^{2} E_{4} A_{4}\right) h_{c 1}^{2} \frac{\partial^{2}}{\partial x^{2}}-G_{c 1} A_{c 1} \\
& a_{23}=\left(-E_{1} A_{1} B_{2} B_{3}+E_{3} A_{3} B_{3} B_{8}+E_{4} A_{4} B_{2} B_{6}\right) h_{c 1} h_{c 2} \frac{\partial^{2}}{\partial x^{2}} \\
& a_{31}=\left(E_{4} A_{4} B_{5} B_{6}-E_{1} A_{1} B_{3} B_{7}-E_{3} A_{3} B_{3} B_{4}\right) h_{c 1} h_{c 2} \frac{\partial^{3}}{\partial x^{3}} \\
& a_{32}=\left(E_{3} A_{3} B_{3} B_{8}-E_{1} A_{1} B_{2} B_{3}+E_{4} A_{4} B_{2} B_{6}\right) h_{c 1} h_{c 2} \frac{\partial^{2}}{\partial x^{2}} \\
& a_{33}=\left(E_{1} A_{1} B_{3}^{2}+E_{3} A_{3} B_{3}^{2}+E_{4} A_{4} B_{6}^{2}\right) h_{c 2}^{2} \frac{\partial^{2}}{\partial x^{2}} \quad-G_{c 2} A_{c 2} \cdot
\end{aligned}
$$

In addition, Hamilton's principle yields the following beam boundary conditions for the general case:

$$
\text { at } x=0:
$$

$$
\left(\frac{\partial w_{1}}{\partial x}, w_{1}, \gamma_{c l}, \gamma_{c 2}\right)=(0,0,0,0)
$$

$$
\begin{aligned}
& \text { at } x=L_{1}: \\
& \begin{aligned}
(D+ & \left.E_{1} A_{1} B_{7}^{2}+E_{3} A_{3} B_{4}^{2}+E_{4} A_{4} B_{5}^{2}\right) \frac{\partial^{2} w_{1}}{\partial x^{2}}+\left(E_{1} A_{1} B_{2} B_{7}-E_{3} A_{3} B_{4} B_{8}\right. \\
& \left.+E_{4} A_{4} B_{2} B_{5}\right) h_{c 1} \frac{\partial \gamma_{c 1}}{\partial x}+\left(E_{4} A_{4} B_{5} B_{6}-E_{1} A_{1} B_{3} B_{7}\right. \\
& \left.-E_{3} A_{3} B_{3} B_{4}\right) h_{c 2} \frac{\partial \gamma_{c 2}}{\partial x}-M_{2}=0
\end{aligned}
\end{aligned}
$$




$$
\begin{gathered}
\left(D+E_{1} A_{1} B_{7}^{2}+E_{3} A_{3} B_{4}^{2}+E_{4} A_{4} B_{5}^{2}\right) \frac{\partial^{3} w_{1}}{\partial x^{3}}+\left(E_{1} A_{1} B_{2} B_{7}-E_{3} A_{3} B_{4} B_{8}\right. \\
\left.+E_{4} A_{4} B_{2} B_{5}\right) h_{c 1} \frac{\partial^{2} \gamma_{c 1}}{\partial x^{2}}+\left(E_{4} A_{4} B_{5} B_{6}-E_{1} A_{1} B_{3} B_{7}\right. \\
\left.-E_{3} A_{3} B_{3} B_{7}\right) h_{c 2} \frac{\partial^{2} \gamma_{c 2}}{\partial x^{2}}+S_{2}=0 \\
\left(E_{3} A_{3} B_{4} B_{8}-E_{1} A_{1} B_{2} B_{7}-E_{4} A_{4} B_{2} B_{5}\right) h_{c 1} \frac{\partial^{2} w_{1}}{\partial x^{2}}-\left(E_{1} A_{1} B_{2}^{2}+E_{3} A_{3} B_{8}^{2}\right. \\
\left.+E_{4} A_{4} B_{2}^{2}\right) h_{c 1}{ }^{2} \frac{\partial \gamma_{c l}}{\partial x}+\left(E_{1} A_{1} B_{2} B_{3}-E_{3} A_{3} B_{3} B_{8}\right. \\
\left.-E_{4} A_{4} B_{2} B_{6}\right) h_{c 1} h_{c 2} \frac{\partial \gamma_{c 2}}{\partial x}=0
\end{gathered}
$$

$$
\begin{gathered}
\left(E_{1} A_{1} B_{3} B_{7}+E_{3} A_{3} B_{3} B_{4}-E_{4} A_{4} B_{5} B_{6}\right) h_{c 2} \frac{\partial^{2} w_{1}}{\partial x^{2}}+\left(E_{1} A_{1} B_{2} B_{3}-E_{3} A_{3} B_{3} B_{8}\right. \\
\left.-E_{4} A_{4} B_{2} B_{6}\right) h_{c 1} h_{\bar{c} 2}\left(E_{1} A_{1} B_{3}^{2}+E_{3} A_{3} B_{3}^{2}\right. \\
\left.+E_{4} A_{4} B_{6}^{2}\right) h_{c 2}^{2} \frac{\partial \gamma_{c 2}}{\partial x}=0
\end{gathered}
$$

\subsubsection{Frequency and loss factor}

To solve Eq. (2-16) for harmonic vibrations, assume the solution to be of the following form:

$$
\left\{\begin{array}{l}
w_{1} \\
\gamma_{c 1} \\
\gamma_{c 2}
\end{array}\right\}=\left\{\begin{array}{c}
w_{1 n} \\
\gamma_{c 1 n} \\
\gamma_{c 2 n}
\end{array}\right\} \mathrm{e}^{k_{s} \cdot \mathrm{x}} \mathrm{e}^{\mathrm{j} \Omega ; \mathrm{t}},
$$

where $k_{n}^{*}$ and $\Omega_{n}^{*}$ denote the unknown complex characteristic value and natural frequency. Substituting Eqs. (2-21) into (2-16), for the eigenvalue problem, we have: 


$$
\left[\begin{array}{lll}
\overline{\mathrm{a}}_{11} & \overline{\mathrm{a}}_{12} & \overline{\mathrm{a}}_{13} \\
\overline{\mathrm{a}}_{21} & \overline{\mathrm{a}}_{22} & \overline{\mathrm{a}}_{23} \\
\overline{\mathrm{a}}_{31} & \overline{\mathrm{a}}_{32} & \overline{\mathrm{a}}_{33}
\end{array}\right]\left[\begin{array}{c}
\mathrm{w}_{1 \mathrm{n}} \\
\gamma_{\mathrm{cln}} \\
\gamma_{\mathrm{c} 2 \mathrm{n}}
\end{array}\right]=\{0\},
$$

where the $\bar{a}_{i j}$ 's are:

$$
\begin{aligned}
& \bar{a}_{11}=-m \Omega_{n}^{* 2}+\left(D+E_{1} A_{1} B_{7}^{2}+E_{3} A_{3} B_{4}^{2}+E_{4} A_{4} B_{5}^{2}\right) k_{n}^{* 4} \\
& \bar{a}_{12}=+\left(E_{1} A_{1} B_{2} B_{7}-E_{3} A_{3} B_{4} B_{8}+E_{4} A_{4} B_{2} B_{5}\right) h_{c 1} k_{n}^{* 3} \\
& \bar{a}_{13}=-\left(E_{1} A_{1} B_{3} B_{7}-E_{3} A_{3} B_{3} B_{4}+E_{4} A_{4} B_{5} B_{6}\right) h_{c 2} k_{n}^{* 3} \\
& \bar{a}_{21}=\left(E_{1} A_{1} B_{2} B_{7}-E_{3} A_{3} B_{4} B_{8}+E_{4} A_{4} B_{2} B_{5}\right) h_{c 1} k_{n}^{* 3} \\
& \bar{a}_{22}=\left(B_{2}{ }^{2} E_{1} A_{1}+B_{8}{ }^{2} E_{3} A_{3}+B_{2}{ }^{2} E_{4} A_{4}\right) h_{c 1}{ }^{2} k_{n}{ }^{* 2}-G_{c 1} A_{c 1} \\
& \bar{a}_{23}=\left(-E_{1} A_{1} B_{2} B_{3}+E_{3} A_{3} B_{3} B_{8}+E_{4} A_{4} B_{2} B_{6}\right) h_{c 1} h_{c 2} k_{n}^{* 2} \\
& \bar{a}_{31}=\left(E_{4} A_{4} B_{5} B_{6}-E_{1} A_{1} B_{3} B_{7}-E_{3} A_{3} B_{3} B_{4}\right) h_{c 2} k_{n}{ }^{* 3} \\
& \bar{a}_{32}=\left(E_{3} A_{3} B_{3} B_{8}-E_{1} A_{1} B_{2} B_{3}+E_{4} A_{4} B_{2} B_{6}\right) h_{c 1} h_{c 2} k_{n}^{* 2} \\
& \bar{a}_{33}=\left(E_{1} A_{1} B_{3}{ }^{2}+E_{3} A_{3} B_{3}{ }^{2}+E_{4} A_{4} B_{6}{ }^{2}\right) h_{c 2}{ }^{2} k_{n}{ }^{* 2}-G_{c 2} A_{c 2} .
\end{aligned}
$$

Hence, the characteristic equation is given by:

$$
\operatorname{det}\left[\begin{array}{lll}
\bar{a}_{11} & \bar{a}_{12} & \bar{a}_{13} \\
\bar{a}_{21} & \bar{a}_{22} & \bar{a}_{33} \\
\bar{a}_{31} & \bar{a}_{32} & \bar{a}_{33}
\end{array}\right]=0
$$

From Eq. (2-23), it can be seen that for any value of $\Omega_{\mathrm{n}}^{*}$, there would be eight values of $k_{n}^{*}$. Let $k_{n 1}^{*}, k_{n 2}^{*}, \ldots, k_{n 8}^{*}$ denote the eight zeros of Eq. (2-23). We then have for the first section $\left(0 \leq \mathrm{x} \leq \mathrm{L}_{1}\right)$

$$
\bar{w}_{1 \mathrm{n}}(\mathrm{x})=\sum_{\mathrm{i}=1}^{8} \overline{\mathrm{A}}_{\mathrm{ni}} \mathrm{e}^{\mathrm{k}: \mathrm{x}}
$$




$$
\begin{aligned}
& \gamma_{c 1 n}(x)=\sum_{i=1}^{8} f_{n i} \bar{A}_{n i} e^{k ; x} \\
& \gamma_{c 2 n}(x)=\sum_{i=1}^{8} g_{n i} \bar{A}_{n i} e^{k ; x},
\end{aligned}
$$

where the $f_{n i}$ 's and $g_{n i}$ 's are defined as follows:

$$
\begin{aligned}
& f_{n i}=\frac{d_{23} d_{31}-d_{21} d_{33}}{d_{22} d_{33}-d_{32} d_{23}} \\
& \mathbf{g}_{n i}=\frac{d_{21} d_{32}-d_{22} d_{31}}{d_{22} d_{33}-d_{32} d_{23}}
\end{aligned}
$$

where

$$
\begin{aligned}
& \mathrm{d}_{11}=-\mathrm{m} \Omega_{\mathrm{n}}{ }^{* 2}+\left(\mathrm{D}+\mathrm{E}_{1} \mathrm{~A}_{1} \mathrm{~B}_{7}{ }^{2}+\mathrm{E}_{3} \mathrm{~A}_{3} \mathrm{~B}_{4}{ }^{2}+\mathrm{E}_{4} \mathrm{~A}_{4} \mathrm{~B}_{5}{ }^{2}\right) \mathrm{k}_{\mathrm{ni}}{ }^{* 4} \\
& d_{12}=+\left(E_{1} A_{1} B_{2} B_{7}-E_{3} A_{3} B_{4} B_{8}+E_{4} A_{4} B_{2} B_{5}\right) h_{c l} k_{n i}^{* 3} \\
& d_{13}=-\left(E_{1} A_{1} B_{3} B_{7}-E_{3} A_{3} B_{3} B_{4}+E_{4} A_{4} B_{5} B_{6}\right) h_{c 2} k_{n i}^{* 3} \\
& d_{21}=\left(E_{1} A_{1} B_{2} B_{7}-E_{3} A_{3} B_{4} B_{8}+E_{4} A_{4} B_{2} B_{5}\right) h_{c 1} k_{n i}^{* 3} \\
& \mathrm{~d}_{22}=\left(\mathrm{B}_{2}{ }^{2} \mathrm{E}_{1} \mathrm{~A}_{1}+\mathrm{B}_{8}{ }^{2} \mathrm{E}_{3} \mathrm{~A}_{3}+\mathrm{B}_{2}{ }^{2} \mathrm{E}_{4} \mathrm{~A}_{4}\right) \mathrm{h}_{\mathrm{cl}}{ }^{2} \mathrm{k}_{\mathrm{ni}}{ }^{2}-\mathrm{G}_{\mathrm{cl}} \mathrm{A}_{\mathrm{cl}} \\
& d_{23}=\left(-E_{1} A_{1} B_{2} B_{3}+E_{3} A_{3} B_{3} B_{8}+E_{4} A_{4} B_{2} B_{6}\right) h_{c 1} h_{c 2} k_{n i}{ }^{* 2} \\
& d_{31}=\left(E_{4} A_{4} B_{5} B_{6}-E_{1} A_{1} B_{3} B_{7}-E_{3} A_{3} B_{3} B_{4}\right) h_{c 2} k_{n i}{ }^{* 3} \\
& d_{32}=\left(E_{3} A_{3} B_{3} B_{8}-E_{1} A_{1} B_{2} B_{3}+E_{4} A_{4} B_{2} B_{6}\right) h_{c 1} h_{c 2} k_{n i}{ }^{* 2} \\
& d_{33}=\left(E_{1} A_{1} B_{3}{ }^{2}+E_{3} A_{3} B_{3}{ }^{2}+E_{4} A_{4} B_{6}{ }^{2}\right) h_{c 2}{ }^{2} k_{n i}{ }^{* 2}-G_{c 2} A_{c 2}
\end{aligned}
$$

The characteristic equation generated from Eq.(2-1) is:

$$
\mathrm{D}_{\mathrm{t} 1} \mathrm{k}_{\mathrm{n}}^{4}-\mathrm{m}_{2} \Omega_{\mathrm{n}}^{* 2}=0
$$

The solution of the above equation yields four roots: $\mathrm{k}_{\mathrm{ng}}, \mathrm{k}_{\mathrm{n} 10^{*}}, \mathrm{k}_{\mathrm{n} 11^{*}}, \mathrm{k}_{\mathrm{n} 12}$. The displacement function in the second section $\left(L_{1} \leq x \leq L\right)$ is then given by 


$$
\begin{aligned}
\bar{w}_{20}(x) & =\sum_{i=9}^{12} \bar{A}_{n i} e^{k_{i: i} x} \\
& =\bar{A}_{n 9} e^{k_{c} x}+\bar{A}_{n 10} e^{-k_{a} x}+\bar{A}_{n 11} e^{j k_{d} x}+\bar{A}_{n 12} e^{-j k_{0} x}
\end{aligned}
$$

Hence, the solutions may be written as:

$$
\begin{aligned}
& w_{1}(x, t)=\sum_{i=1}^{8} \bar{A}_{n i} e^{x_{i i}^{\cdot} x} e^{j \Omega i t}
\end{aligned}
$$



$$
\begin{aligned}
& w_{2}(x, t)=\left(\AA_{n 9} e^{k_{n} x}+I_{n 10} e^{-k_{n} x}+\AA_{n 11} e^{j k_{a} x}\right. \\
& \left.+\bar{A}_{\mathrm{n} 12} \mathrm{e}^{\left.-\mathrm{j} \mathrm{k}_{\mathrm{n}}^{\mathrm{x}}\right)}\right) \mathrm{e}^{\mathrm{i} \Omega_{\mathrm{n}}^{*} \mathrm{t}} \quad \mathrm{L}_{1} \leq \mathrm{x} \leq \mathrm{L}
\end{aligned}
$$

There are 12 unknowns for which Eqs. (2-2), and (2-17)-(2-20) yield 10 boundary conditions. Also, the axial forces, $\mathrm{P}_{3}$ and $\mathrm{P}_{4}$, of the damped part at $\mathrm{x}=\mathrm{L}_{1}$ must be zero, i.e.,

$$
\begin{aligned}
& P_{3}=E_{3} A_{3} \frac{\partial u_{3}}{\partial x}=0 \\
& P_{4}=E_{4} A_{4} \frac{\partial u_{4}}{\partial x}=0
\end{aligned}
$$

Hence, we have a total of twelve equations to determine the 12 unknowns.

Substituting Eqs. (2-26) and (2-28) into the boundary conditions and supplementary conditions, we obtain a 12th-order characteristic determinant.

$$
\operatorname{det}[\mathrm{Q}]=0
$$


The elements of the matrix $[Q]$ are:

$$
\begin{aligned}
& Q_{1, i}= \begin{cases}k_{n i}^{*} & i=1,2, \ldots .8 \\
0 & i=9,10,11,12\end{cases} \\
& Q_{2, i}= \begin{cases}1 & i=1,2, \ldots .8 \\
0 & i=9,10,11,12\end{cases} \\
& Q_{3, i}= \begin{cases}f_{n i} & i=1,2, \ldots .8 \\
0 & i=9,10,11,12\end{cases} \\
& Q_{4, i}= \begin{cases}g_{n i} & i=1,2, \ldots .8 \\
0 & i=9,10,11,12\end{cases}
\end{aligned}
$$

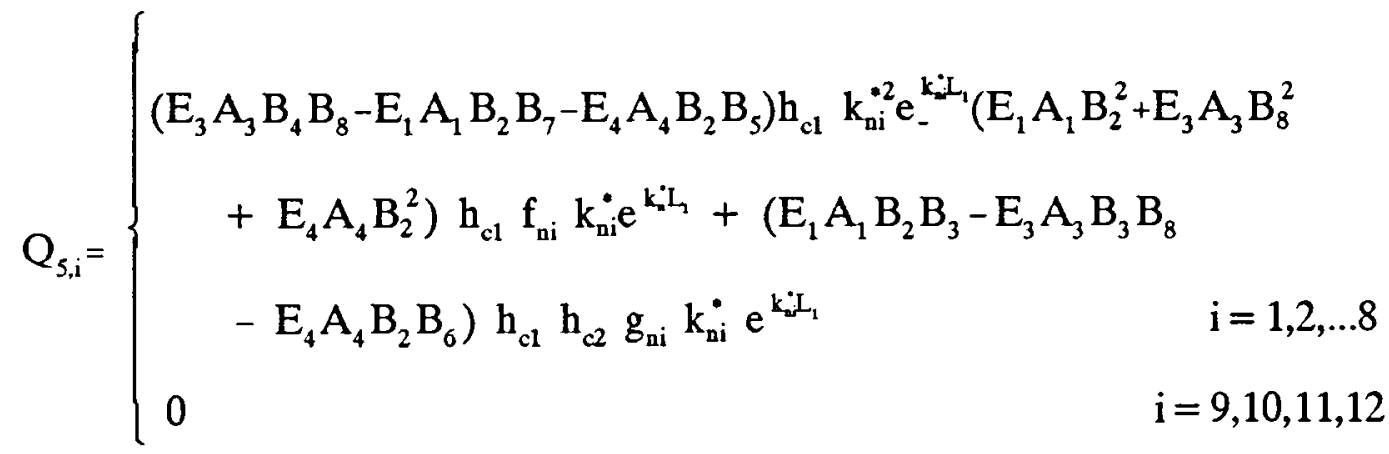

$$
\begin{aligned}
& Q_{6, i}=\left\{\begin{array}{cc}
\left(E_{1} A_{1} B_{3} B_{7}-E_{3} A_{3} B_{3} B_{4}-E_{4} A_{4} B_{5} B_{6}\right) h_{c 2} k_{n i}^{* 2} e^{k_{i n}^{*} h_{1}}+\left(E_{1} A_{1} B_{2} B_{3}\right. \\
\left.-E_{3} A_{3} B_{3} B_{8}-E_{4} A_{4} B_{2} B_{6}\right) h_{c 1} h_{c 2} f_{n i} k_{n i}^{\cdot} e^{k_{j} \cdot L_{1}}-\left(E_{1} A_{1} B_{3}^{2}\right. \\
\left.+E_{3} A_{3} B_{3}^{2}+E_{4} A_{4} B_{6}^{2}\right) h_{c 2}^{2} g_{n i} k_{n i}^{\cdot} e^{k_{2}^{\prime} L_{1}} & i=1,2, \ldots 8 \\
0 & i=9,10,11,12
\end{array}\right.
\end{aligned}
$$

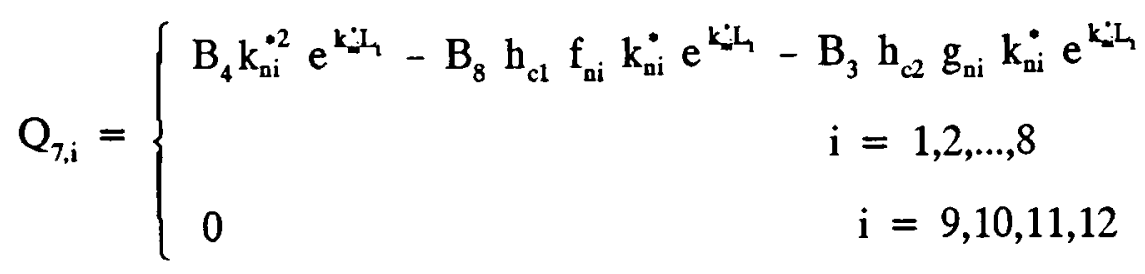




$$
\begin{aligned}
& Q_{8, i}=\left\{\begin{array}{rr}
B_{5} k_{n i}^{* 2} e^{k_{L} L_{1}}+B_{2} h_{c 2} f_{n i} k_{n i}^{*} e^{k_{\alpha}^{*} L_{1}}+B_{6} h_{c 2} g_{n i} k_{n i}^{*} e^{k_{-1}^{*} L_{1}} \\
i=1,2, \ldots, 8 \\
0 \quad i=9,10,11,12
\end{array}\right. \\
& Q_{9, i}=\left(D+E_{3} A_{3} B_{7}^{2}+E_{3} A_{3} B_{4}^{2}+E_{4} A_{4} B_{5}^{2}\right) k_{n i}^{* 2} e^{k_{4} d_{1}}+\left(E_{1} A_{1} B_{2} B_{7}-E_{3} A_{3} B_{4} B_{8}\right. \\
& \left.+E_{4} A_{4} B_{2} B_{5}\right) h_{c l} f_{n i} k_{n i}^{*} e^{k_{i i}^{\prime} L}+\left(E_{4} A_{4} B_{5} B_{6}-E_{1} A_{1} B_{3} B_{7}\right. \\
& \left.-E_{3} A_{3} B_{3} B_{4}\right) h_{c 2} g_{n i} k_{n i}^{*} e^{k_{i}^{*} L_{1}} \\
& Q_{9,9}=D_{11} k_{n}^{2} e^{k_{n} L_{1}} \\
& Q_{9,10}=D_{t 1} k_{n}^{2} e^{-k_{n} L_{1}} \\
& \mathrm{Q}_{9,11}=\mathrm{D}_{\mathfrak{t 1}} \mathbf{k}_{\mathrm{n}}^{2} \mathrm{e}^{\mathrm{j} \mathbf{k}_{\mathrm{n}} \mathrm{L}_{1}} \\
& \mathrm{Q}_{9,12}=\mathrm{D}_{\mathrm{u} 1} \mathrm{k}_{\mathrm{n}}^{2} \mathrm{e}^{-\mathrm{j} \mathrm{k}_{\mathrm{n}} \mathrm{L}_{1}} \\
& Q_{10, i}=\left(D+E_{1} A_{1} B_{7}^{2}+E_{3} A_{3} B_{4}^{2}+E_{4} A_{4} B_{5}^{2}\right) k_{a i}^{* 3} e^{k-L_{4}}+\left(E_{1} A_{1} B_{2} B_{7}-E_{3} A_{3} B_{4} B_{8}\right. \\
& \left.+E_{4} A_{4} B_{2} B_{5}\right) h_{c l} f_{n i} k_{n i}^{* 2} e^{k_{s i}^{*} L_{4}}+\left(E_{4} A_{4} B_{5} B_{6}-E_{1} A_{1} B_{3} B_{7}\right. \\
& \left.-\mathrm{E}_{3} \mathrm{~A}_{3} \mathrm{~B}_{3} \mathrm{~B}_{4}\right) \mathrm{h}_{\mathrm{c} 2} \mathrm{~g}_{\mathrm{ni}} \mathrm{k}_{\mathrm{ni}}^{* 2} \mathrm{e}^{\mathrm{k}_{\mathrm{m}} \cdot \mathrm{L}_{4}} \quad \mathrm{i}=1,2, \ldots 8 \\
& \mathrm{Q}_{10,9}=\mathrm{D}_{\mathbf{1 1}} \mathrm{k}_{\mathrm{n}}^{3} \mathrm{e}^{\mathrm{k}_{\mathrm{n}} \mathrm{L}_{1}} \\
& \mathrm{Q}_{10,10}=-\mathrm{D}_{\mathrm{t} 1} \mathrm{k}_{\mathrm{n}}^{3} \mathrm{e}^{-\mathrm{k}_{\mathrm{n}} \mathrm{L}_{1}} \\
& Q_{10,11}=-j D_{t} k_{n}^{3} e^{j k_{n} L_{1}} \\
& \mathrm{Q}_{10,12}=\mathrm{j} \mathrm{D} \mathrm{n} \mathrm{k}_{\mathrm{n}}^{3} \mathrm{e}^{\mathrm{j} \mathrm{k}_{\mathrm{n}} \mathrm{L}_{1}} \\
& \mathrm{Q}_{11, \mathrm{i}}=0 \quad \mathrm{i}=1,2, \ldots 8 \\
& \mathrm{Q}_{11,9}=\mathrm{e}^{\mathrm{k}_{\mathrm{n}} \mathrm{L}} \\
& Q_{11,10}=e^{-k_{n} L} \\
& \mathrm{Q}_{11.11}=\mathrm{e}^{\mathrm{j} \mathbf{k}_{\mathrm{n}} \mathrm{L}} \\
& i=1,2, \ldots 8
\end{aligned}
$$




$$
\begin{array}{ll}
\mathrm{Q}_{11,12}=-\mathrm{e}^{-j k_{\mathrm{n}} \mathrm{L}} & \mathrm{i}=1,2, \ldots 8 \\
\mathrm{Q}_{12, \mathrm{i}}=0 & \\
\mathrm{Q}_{12,9}=\mathrm{e}^{\mathrm{k}_{\mathrm{n}} \mathrm{L}} & \\
\mathrm{Q}_{12,10}=-\mathrm{e}^{-\mathrm{k}_{\mathrm{n}} \mathrm{L}} & \\
\mathrm{Q}_{12,11}=-\mathrm{j} \mathrm{e}^{\mathrm{j} \mathrm{k}_{\mathrm{n}} \mathrm{L}} & \mathrm{j} \mathrm{e}^{-j \mathrm{k}_{\mathrm{n}} \mathrm{L}}
\end{array}
$$

Equations (2-23), (2-25), and (2-31) are nonlinear, complex valued equations for unknowns, $\Omega_{\mathrm{n}}^{*}, \mathrm{k}_{\mathrm{n}}^{*}, \mathrm{k}_{\mathrm{n}}$. The problem is solved numerically using a trial and error technique in which the beginning values are assumed to be those of an uncovered Euler beam. Complex double precision has been used to obtain the results. The complex frequency factor, $\mathrm{p}^{*}$, and the real frequency factor, $\mathrm{p}$, and the loss factor, $\eta$, of the beam are related by:

$$
\left.\begin{array}{l}
\mathrm{p}^{*}=\Omega^{*} \mathrm{t}_{0}=\mathrm{p}(1+\mathrm{j} \eta)^{1 / 2} \\
\mathrm{p}=\Omega \mathrm{t}_{0}=\sqrt{\operatorname{Re}\left(\mathrm{p}^{* 2}\right)} \\
\eta=\operatorname{Im}\left(\mathrm{p}^{* 2}\right) / \operatorname{Re}\left(\mathrm{p}^{* 2}\right)
\end{array}\right\}
$$

where $t_{0}=\sqrt{\mathrm{mL}^{4} / \mathrm{D}}$. 
2.1.4 Comparison of equation (2-16) with Rao (1978)

In the case of a single sandwich-type beam, we have

$$
\mathrm{E}_{4} \mathrm{~A}_{4}=\mathrm{h}_{4}=\mathrm{h}_{\mathrm{c} 2}=\mathrm{G}_{\mathrm{c} 2}=0 \text {. }
$$

If we use the conditions of Eq. (2-33), then Eq. (2-16) yields the following form:

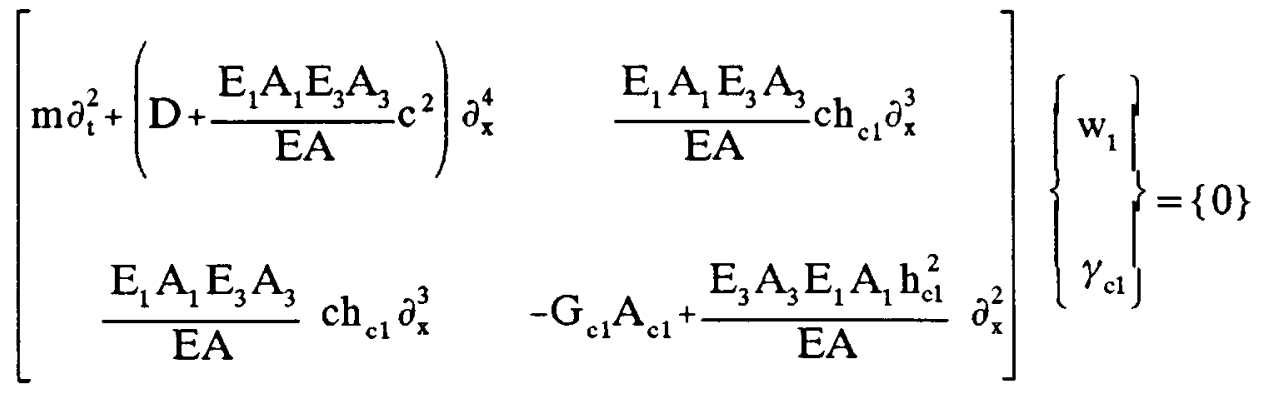

where $c=h_{c 1}+\frac{1}{2}\left(h_{1}+h_{3}\right)$.

If we select the following parameters used by Rao (1978)

$$
\begin{aligned}
& \bar{x}=x / L \\
& \bar{t}=t / t_{o} \quad t_{0}=\sqrt{\mathrm{mL}^{4} / D} \\
& Y=\frac{c^{2} E_{1} A_{1} E_{3} A_{3}}{D E A} \\
& g=\frac{G_{c 1} A_{c l} L^{2}}{h_{c 1}^{2}} \quad \frac{E_{1} A_{1}+E_{3} A_{3}}{E_{1} A_{1} E_{3} A_{3}} \\
& u=\frac{h_{c 1} L}{c} \gamma_{c 2}
\end{aligned}
$$

then Eq. (2-34) can be simplified to the following form: 


$$
\begin{gathered}
\frac{\partial^{2} \mathrm{w}}{\partial \overline{\mathrm{t}}^{2}}+(1+\mathrm{Y}) \frac{\partial^{4} \mathrm{w}}{\partial \overline{\mathrm{x}}^{4}}-\mathrm{Y} \frac{\partial^{3} \mathrm{u}}{\partial \overline{\mathrm{x}}^{3}}=0 \\
\mathrm{Y} \frac{\partial^{3} \mathrm{u}}{\partial \overline{\mathrm{x}}^{3}}+\mathrm{g} \cdot \mathrm{Yu}-\mathrm{Y} \frac{\partial^{2} \mathrm{u}}{\partial \overline{\mathrm{x}}^{2}}=0
\end{gathered}
$$

This is equation obtained by Rao (1978).

2.1.5 Numerical results and discussion

The parameters chosen are the geometrical properties, the physical properties, mass density, and core loss factor. Their effects can be seen in Figures 2.3-2.11. The values chosen for the computation, unless stated otherwise, are:

$$
\begin{aligned}
\mathrm{E}_{1}=\mathrm{E}_{3}=\mathrm{E}_{4}=20.6 \times 10^{10} \mathrm{~N} / \mathrm{m}^{2} ; \mathrm{G}_{\mathrm{cl}}=\mathrm{G}_{\mathrm{c} 2}=0.42 \times 10^{10} \mathrm{~N} / \mathrm{m}^{2} ; \mathrm{L}=0.5 \mathrm{~m}, \mathrm{~h}_{1}=0.02 \mathrm{~m} ; \\
\rho_{1}=\rho_{2}=\rho_{3}=7850 \mathrm{Kg} / \mathrm{m}^{3} ; \rho_{\mathrm{cl}}=\rho_{\mathrm{c} 2}=3140 \mathrm{Kg} / \mathrm{m}^{3} ; \mathrm{h}_{3}=\mathrm{h}_{4} ; \mathrm{h}_{\mathrm{cl}}=\mathrm{h}_{\mathrm{c} 2} .
\end{aligned}
$$

These values are identical to those chosen by Rao (1978).

Effects of the geometrical parameters

The effect of the damping layer length on the real frequency factor, $p$, is shown in Figure 2.3 for $h_{3} / h_{1}=0.5$. It appears that initially the real frequency factor for the first mode increases as the damping length increases. This is contrary to expectations; we would expect that as coverage increases, the real frequency factor should decrease, which occurs after $\mathrm{L}_{1} / \mathrm{L}=0.5$. We conclude that the constraining layer, whose length is the same as the damping layer and whose parameters are the 


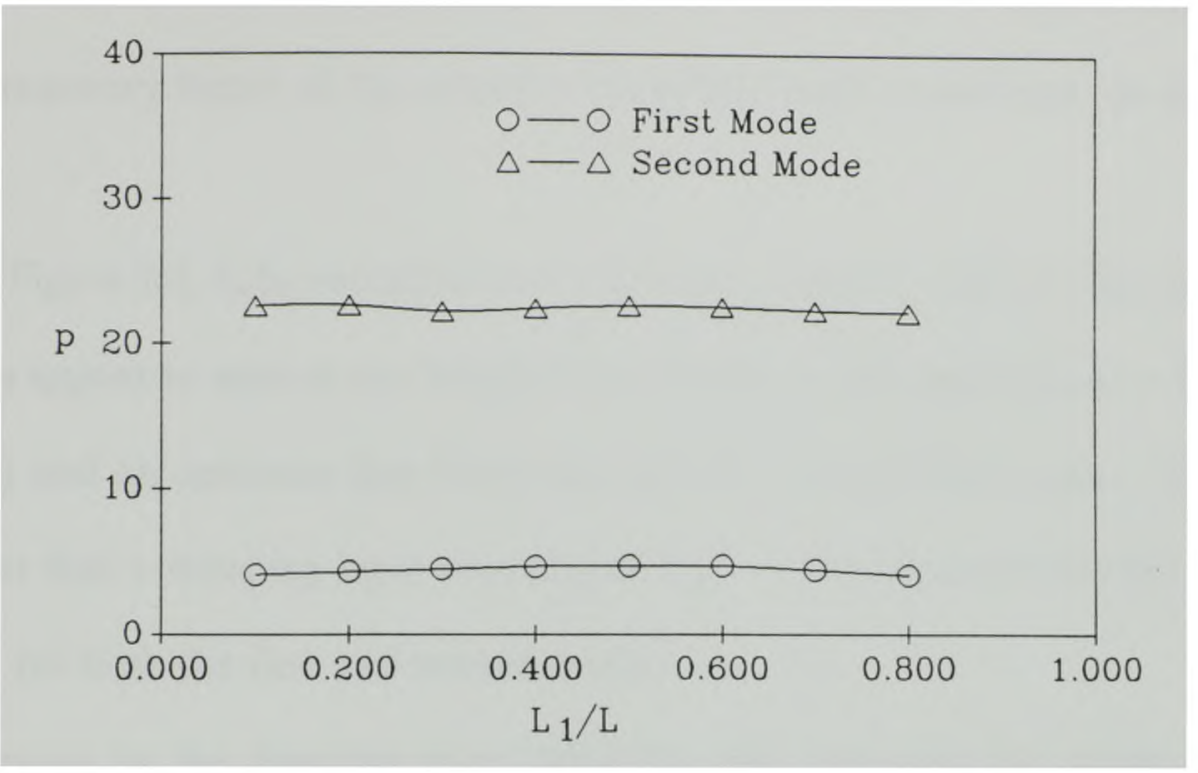

Figure 2.3 Damping layer coverage length versus frequency factor

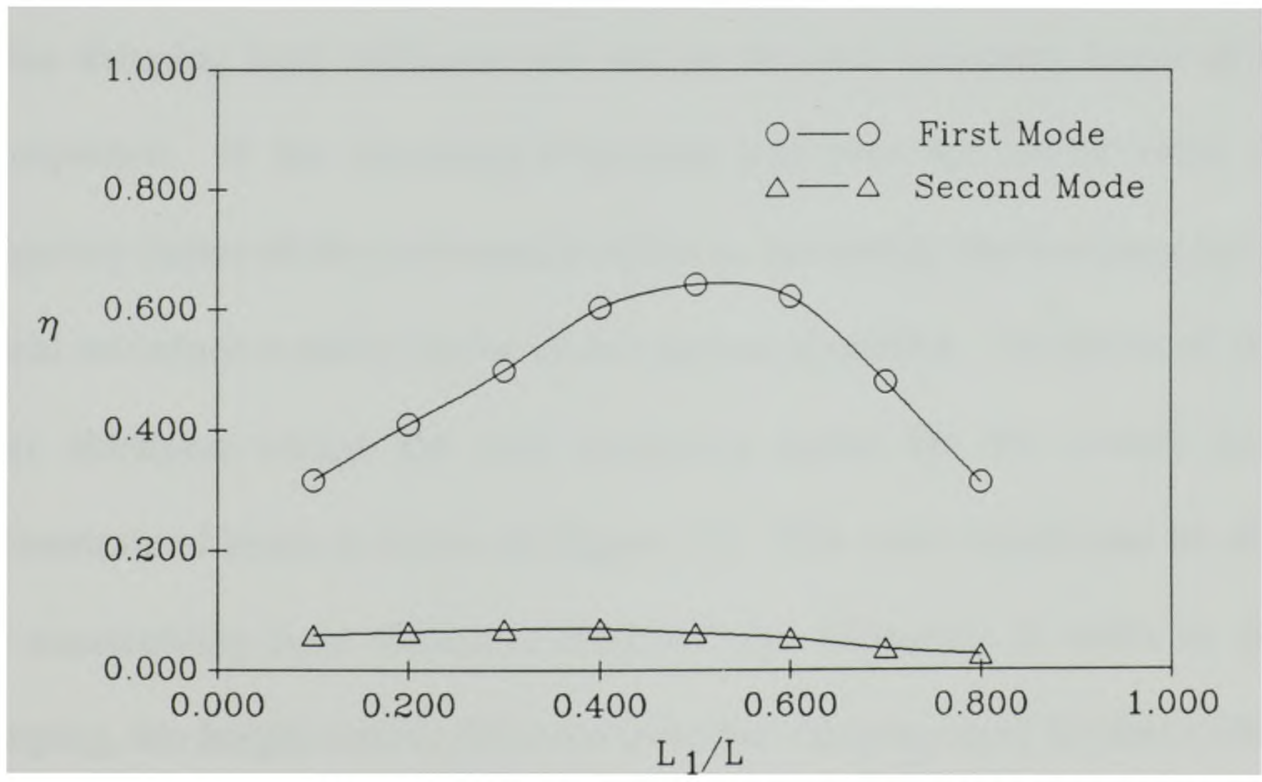

Figure 2.4 Coverage length ratio versus system loss factor 
same as those of the beam, acts in a manner that moves the fixed end of the beam closer to the free end, shortening the effective beam length. This in turn causes the real frequency factor of the effective fixed-free beam to increase, as shown in the figure.

In Figure $2.4, h_{3} / h_{1}$ was taken as 0.5 and $h_{c 1} / h_{1}$ was 0.5 . We see that optimum loss factors appear to exist at two length ratios for the second mode $\left(\mathrm{L}_{1} / \mathrm{L}=0.2\right.$ and $\mathrm{L}_{1} / \mathrm{L}$ $=0.5$ ) and an optimum loss factor at $\mathrm{L}_{1} / \mathrm{L}=0.5$ for the first mode. These results suggest that a damping layer covering of $L_{1} / L=0.5$ can optimize the system loss factor for both the first and second modes.

Increase in the damping layer thickness also increases the system loss factor (Figure 2.5), as expected, until a maximum value is reached. For different values of $\mathrm{L}_{1} / \mathrm{L}$ the optimum value of $\eta$ occurs when $\mathrm{h}_{\mathrm{c} 2} / \mathrm{h}_{1}$ is in the range 0.8 to 1.0 . Increase in the damping layer thickness will reduce the real frequency factor of the system, as expected. If the operating frequency was near the lowest value of the real frequency factor of the unconstrained beam, increasing the damping layer thickness would introduce a safety factor in the system operation. Variation of the damping layer thickness versus the real frequency factor for the lowest mode of the unconstrained beam is shown in Figure 2.6. The same trends can be observed for the constraining layer thickness (Figure 2.7). However, in order to obtain good damping, the height ratio is different from the damping layer thickness ratio. If $h_{3} / h_{1}$ is less than 0.4 , increasing the damping layer coverage will increase the system loss factor. 


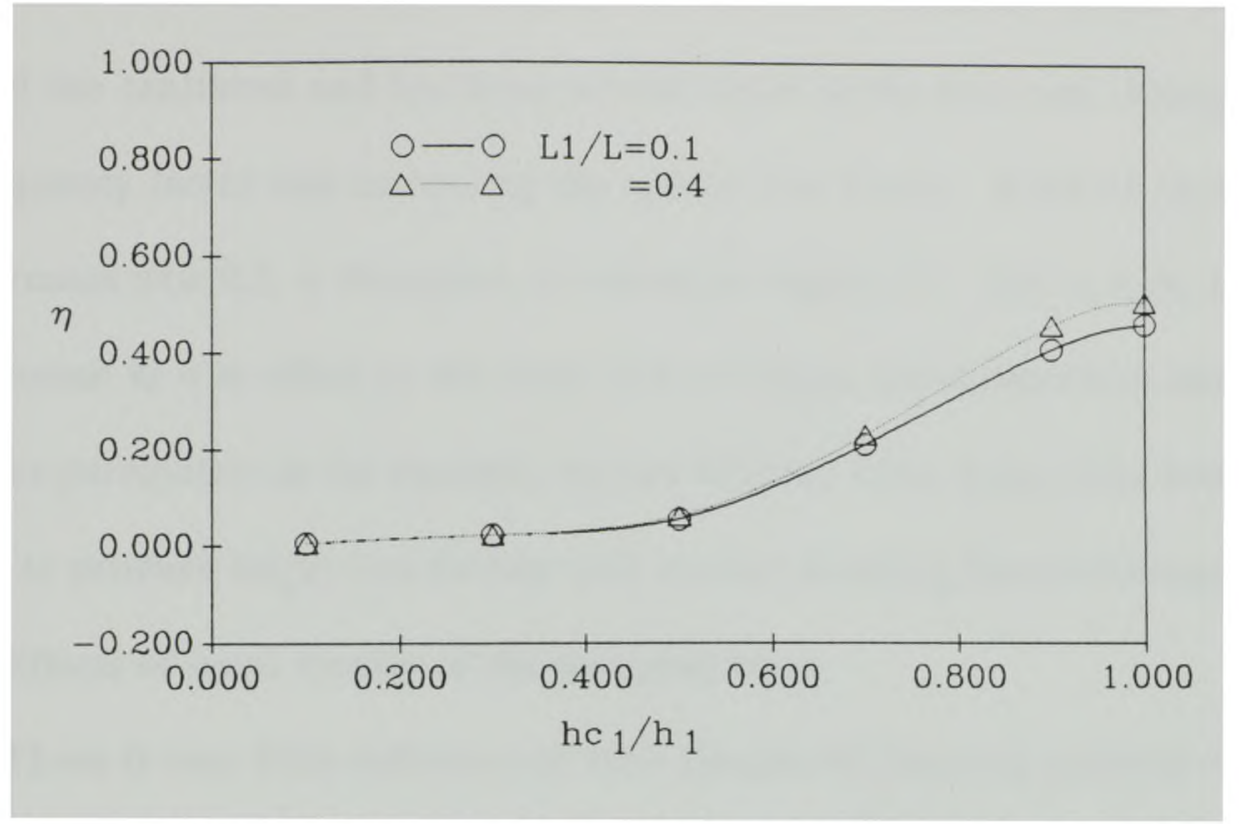

Figure 2.5 Damping layer thickness versus system loss factor

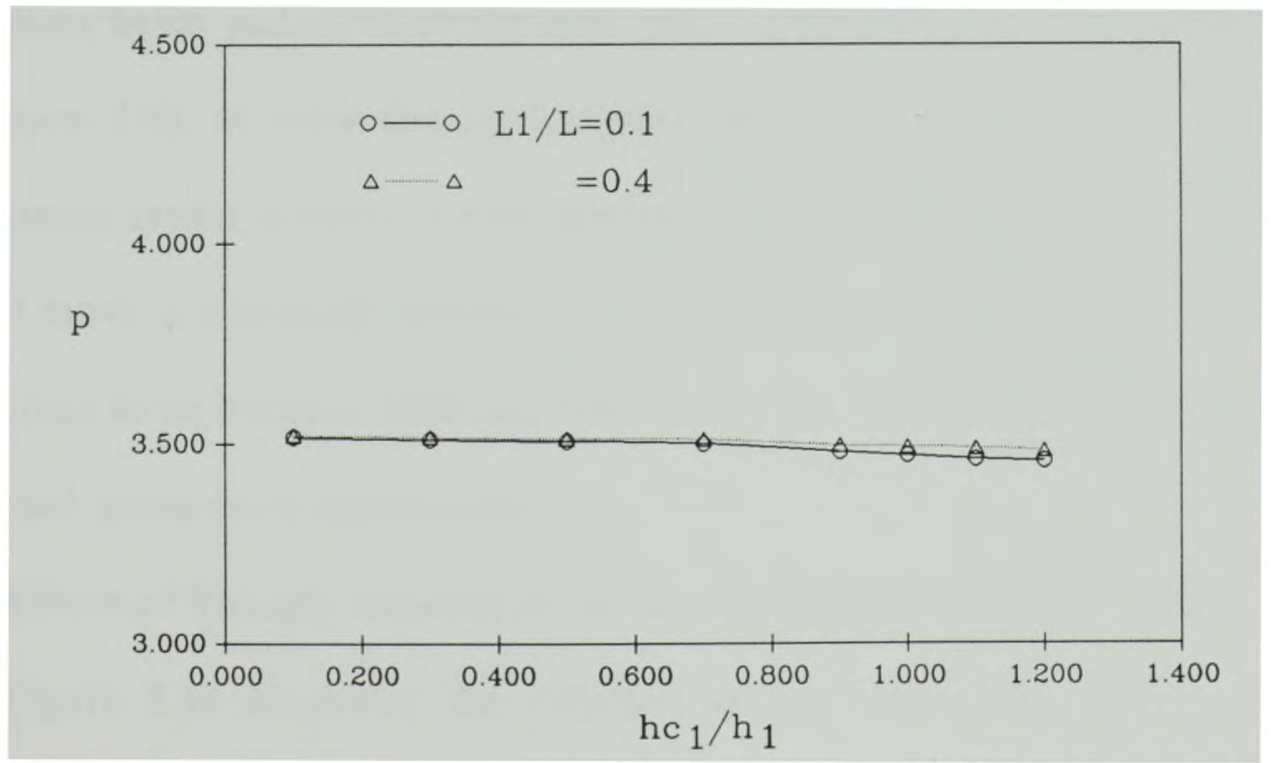

Figure 2.6 Damping layer thickness ratio versus frequency factor 
In this case, the viscoelastic layer's effect $\left(h_{c 1} / h_{1}=0.5\right)$ dominates, thereby causing an increase in the loss factor. For $h_{3} / h_{1}>0.6$, the same situation occurs. The beam acts as if the cantilever end has been moved closer to the free end, driving up the real frequency factor and increasing the system loss factor. If $0.4<\mathrm{h}_{3} / \mathrm{h}_{1}<0.6$, as $\mathrm{L}_{1} / \mathrm{L}$ increases past $0.5, \eta$ decreases, as shown in Figure 2.7. But as $h_{3} / h_{1}$ increases, the decrease in $\eta$ is offset by the fixed end condition effect described above. For the given parameters in the example, we can take the ratio, $h_{3} / h_{1}$, to be between 0.4 and 0.6 to produce larger loss factors with smaller damping layer coverage.

Effects of mass density of the damping layer

There is very little influence of mass density of damping material on the system real frequency factor (Figure $2.8, \mathrm{~L}_{1} / \mathrm{L}=0.2$ ). As mentioned previously, two possible reasons are that the damping layer mass is about $6 \%$ of the whole mass, and that primary beam and constraining layer play a principal role. For system loss factors (Figure 2.9), as mass density becomes larger, the inertia of the damping layer becomes harder to move, not dissipating energy. Thus, the system loss factor for the first mode is minimally affected. For our parameters, if the value of mass density is taken to be between 1000 and $4000 \mathrm{~kg} / \mathrm{m}^{3}$, the values of the loss factor affect the second mode more significantly.

Effects of Young's modulus of the constraining layer on the system

Figure 2.10 illustrates the variation of the system loss factor with Young's modulus. It can be seen that if the value of Young's modulus of the constraining layer $\left(E_{3}, E_{4}\right)$ is too small, there will be very small system loss factor. This, in effect, 


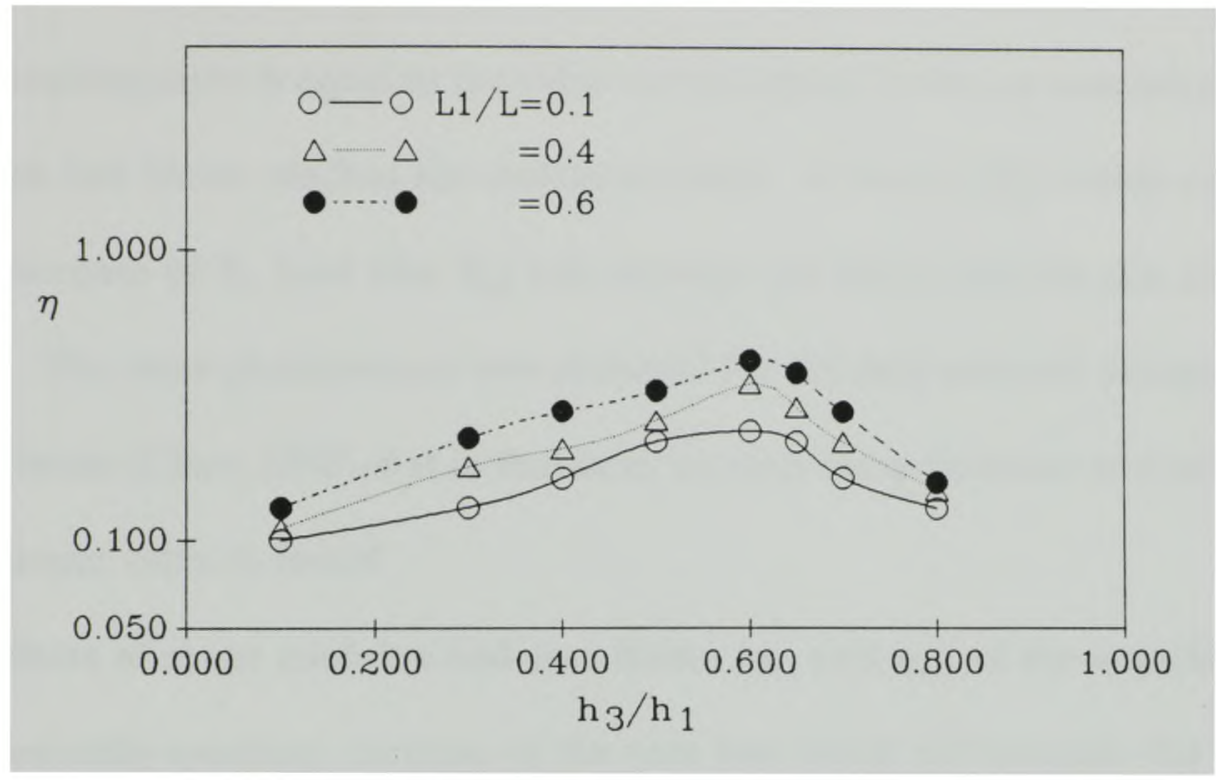

Figure 2.7 Constraining layer thickness ratio versus system loss factor

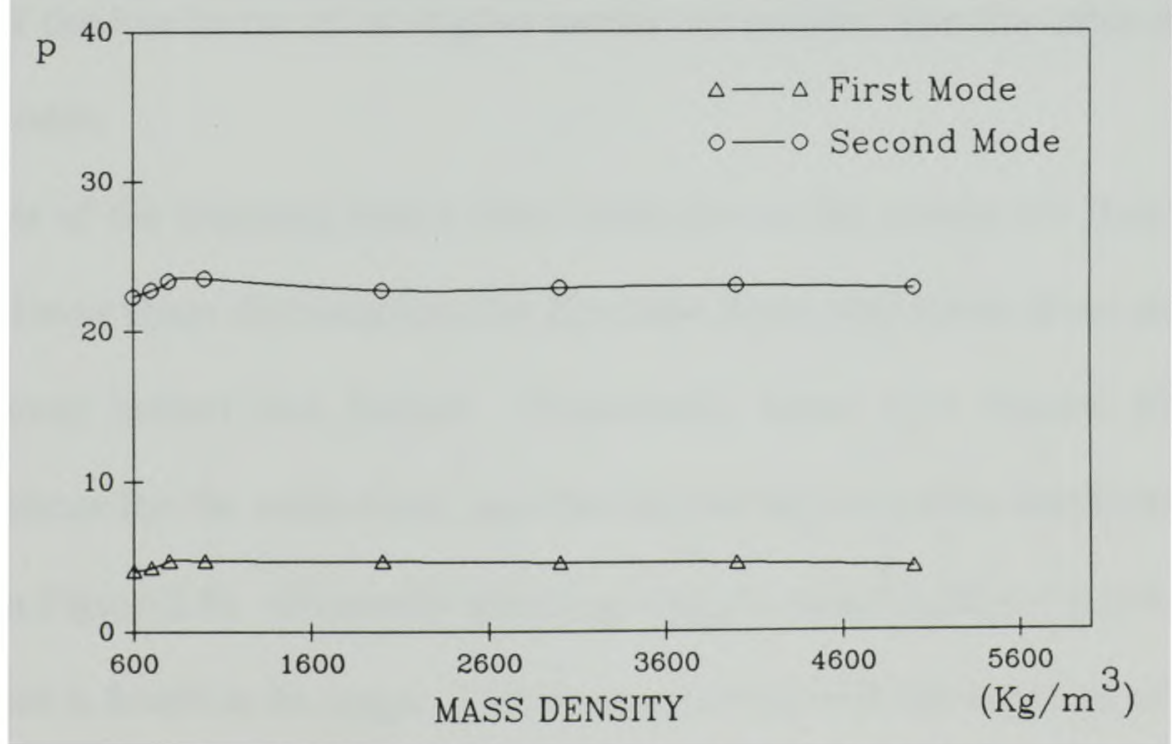

Figure 2.8 Mass density versus frequency factor 
implies that we have an unconstrained beam. As mentioned in Chen (1986), the vibration reduction effect will be small. If the value of Young's modulus of the constraining layer is equal to the value of the original beam, or somewhat higher, the system loss factor reaches the maximum value. It seems that within certain limits, the increase of $E_{3}$ (and also $E_{4}$ ) will increase the shear deformation and the value of $\eta$. The same phenomenon was observed for the fully covered damped sandwichtype beam (Chen, 1986), but in that case, no such sharp decrease of $\eta$ exists after the maximum value is found.

Effects of shear modulus and loss factor $\left(\eta_{\mathrm{c} 1}\right.$ and $\left.\eta_{\mathrm{c} 2}\right)$ of the damping layers

Generally speaking, increase of the core loss factor will increase the value of the system loss factor, as noted in Figure 2.11. The same results were obtained for the fully covered damped sandwich-type beam (Chen 1986). As noted in Figure 2.4, the values of the loss factor of the higher modes are smaller than the value of the lower order modes.

Effects of the damping layer's shear modulus on the system are that higher $G_{c}$ 's require lower shear deformations for the same force, and lower shear deformations mean lower system loss factors. Conversely, lower $G_{c}$ 's require higher shear deformations for the same force, and this means higher system loss factors. This is shown in Figure 2.11. Generally speaking, if $G_{c 1} / E_{1}$ (and $\left.G_{c 2} / E_{1}\right)<0.004$, the system loss factor is found to be large. This compares well with the range of values, 0.001 0.0017, found in Lall et al.(1987) . 




Figure 2.9 Mass density versus system loss factor

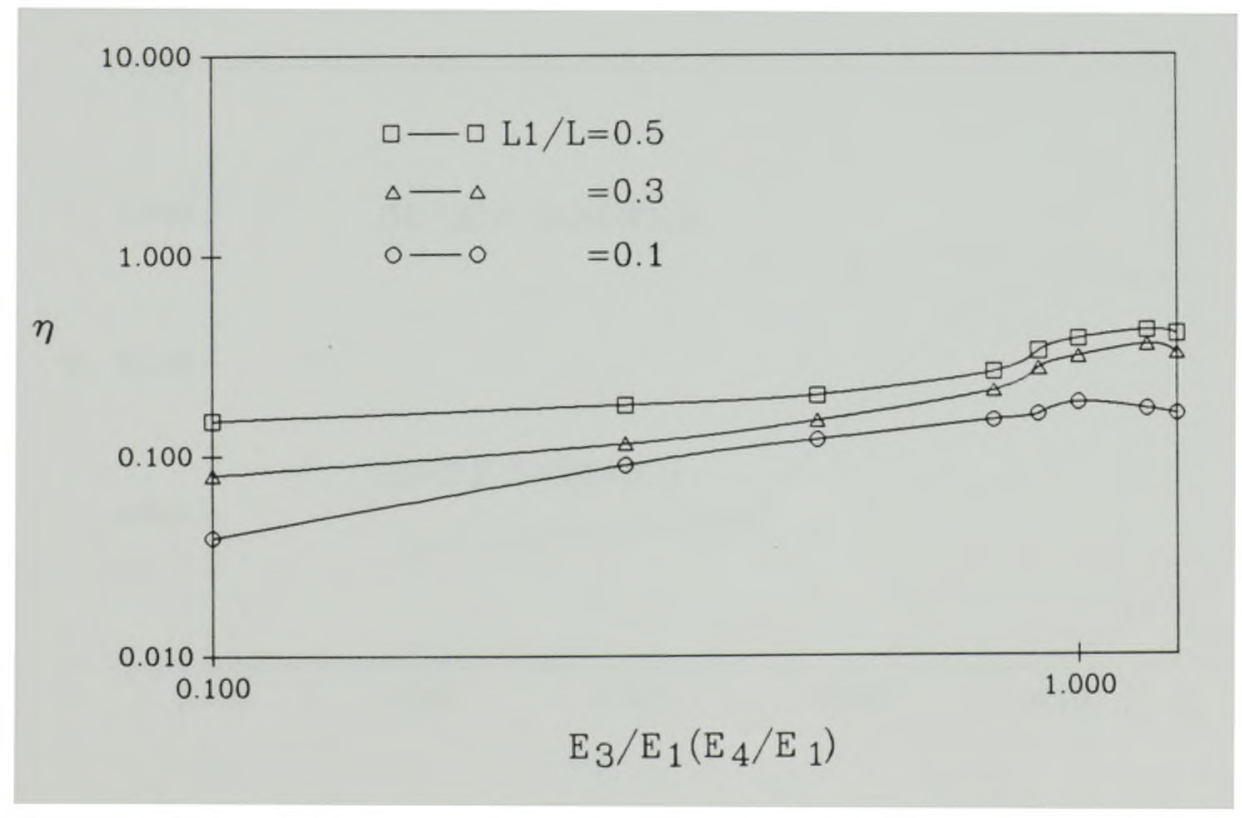

Figure 2.10 Young's modulus ratio versus system loss factor 


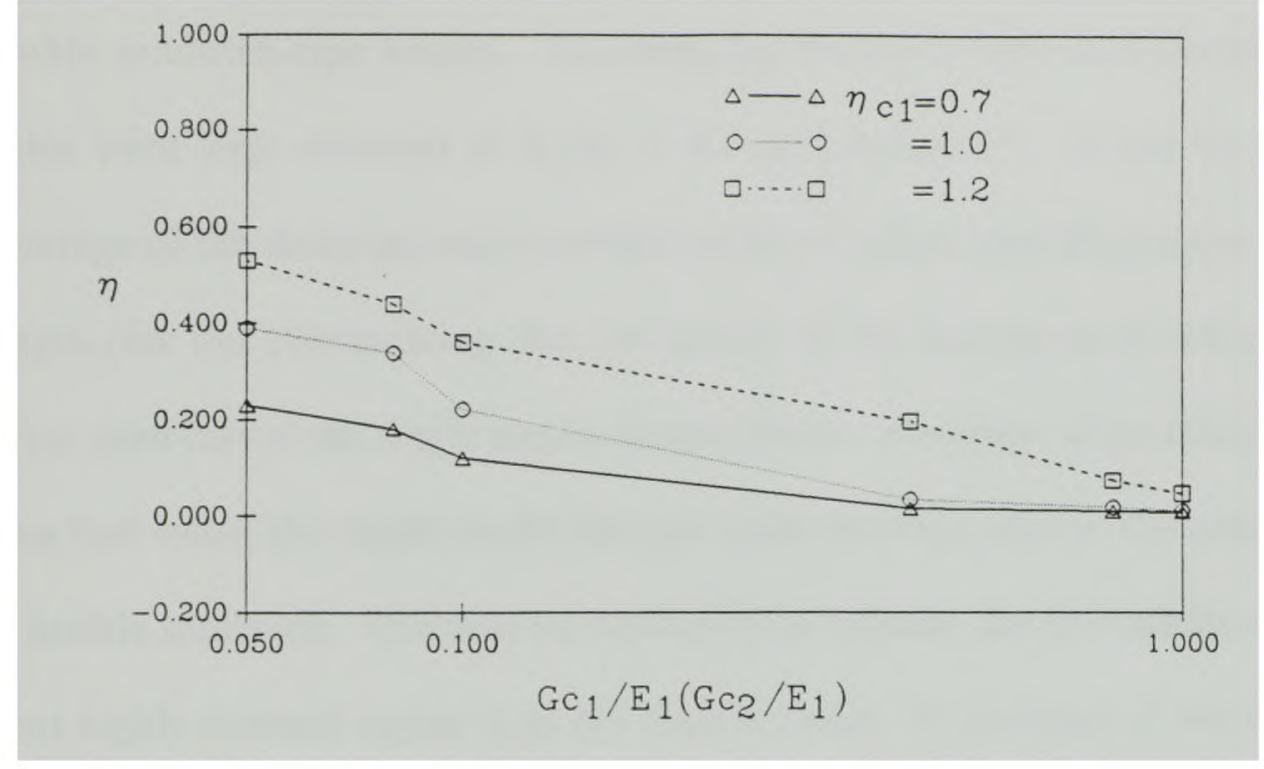

Figure 2.11 Damping layer shear modulus ratio versus system loss factor

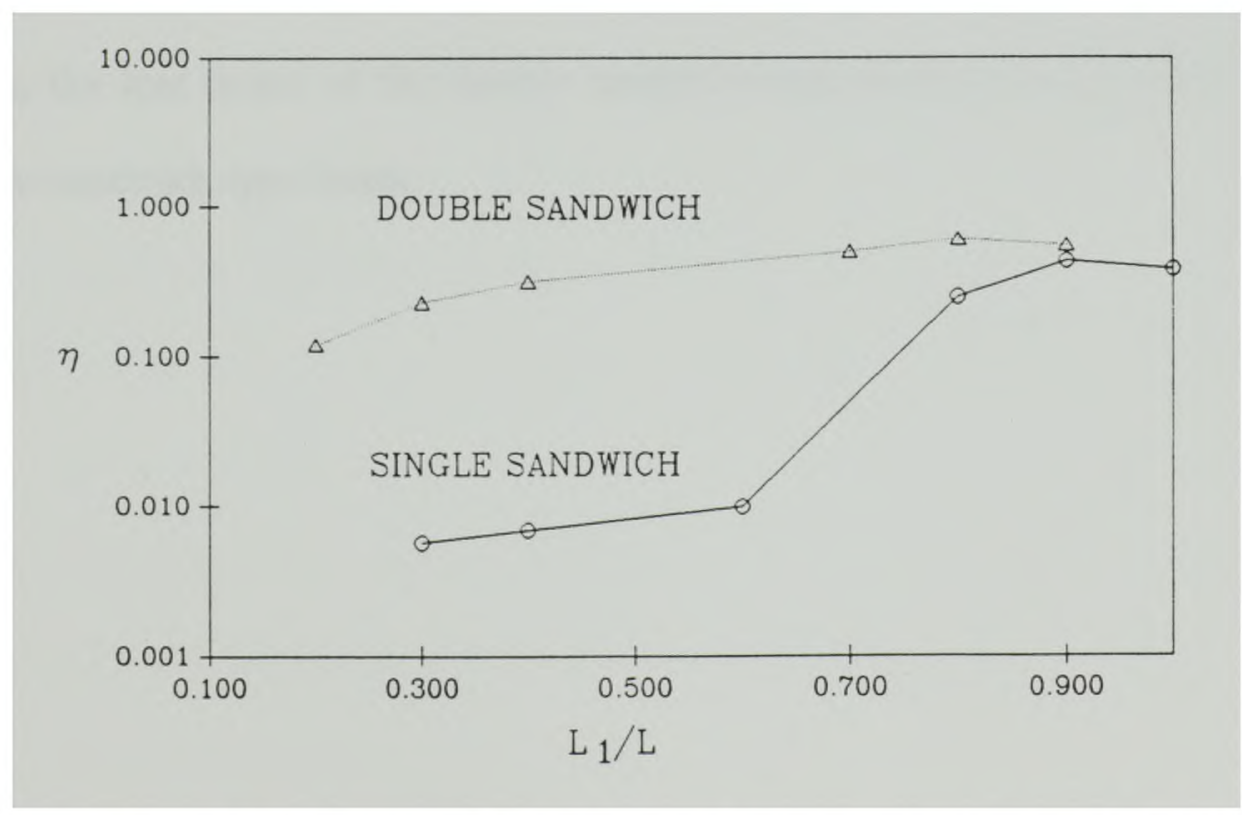

Figure 2.12 Comparisons between single ans double sandwich beam 
Comparison of the double sandwich beam with the single sandwich beam

Figure 2.12 provides a comparison of the system loss factor between single and double sandwich-type beams. The damping thickness ratio and constraining layer ratios were kept constant at $h_{c l} / h_{2}=0.5$ and $h_{3} / h_{1}=0.5$. It can be seen that if coverage of the damping and constraining layers is less than 80 percent of the beam length (for our parameters), the loss factor of the double sandwich-type beam is larger than that of the single sandwich-type beam. However, if the coverage is larger than that value, the single sandwich-type beam provides almost the same loss factor as double sandwich. This may be explained as follows: for the cantilever beam, the most highly stressed region is at the clamped end. If coverage of the damping and constraining layers is small, the double sandwich-type beam gives more energy dissipation than the single sandwich-type beam because of the two damping layers. Thus, the loss factor of the double sandwich-type beam is larger than that of the single sandwich-type beam. 
2.2 Vibration characteristics of partially covered double sandwich cantilever beam with concentrated mass at the free end

In the case when robot arms or manipulators are carring weight, the model given in section 2.1 must be modified. This section discusses the partially covered double sandwich cantilever beam with concentrated mass at the free-end. The equations of motion for the system are derived and the resonant frequency and system loss factor for different geometrical and physical parameters are also discussed.

\subsubsection{Equations of motion}

The double sandwiched cantilever with a concentrated mass at the free-end is shown in Fig. 2.13. To make the mathematical method tractable, the following assumptions are made in the analysis:

1). The beam deflection is small and uniform across any section;

2). The primary beam and the upper and lower constraining beams are assumed to be isotropic;

3). The longitudinal and rotatory inertia effects at the beam are neglected;

4). The damping layers which carry shear but no direct stress are assumed to be linear viscoelastic;

5). No slip occurs at the interface between the layers;

6). The mass at the end effector can be modeled as a concentrated mass. 


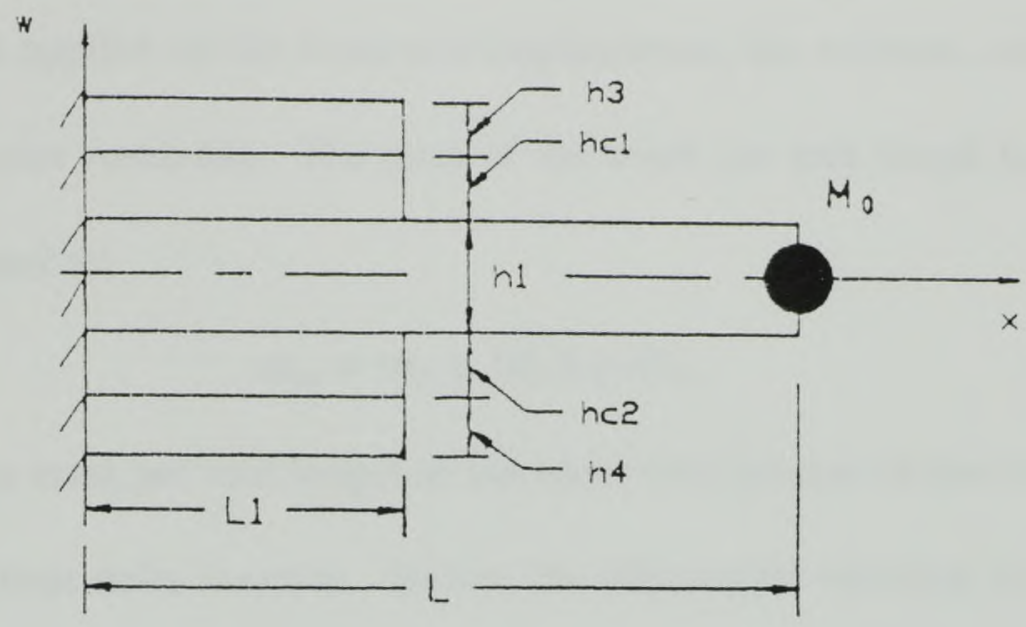

(a) The Covered Beam with Tip Mass

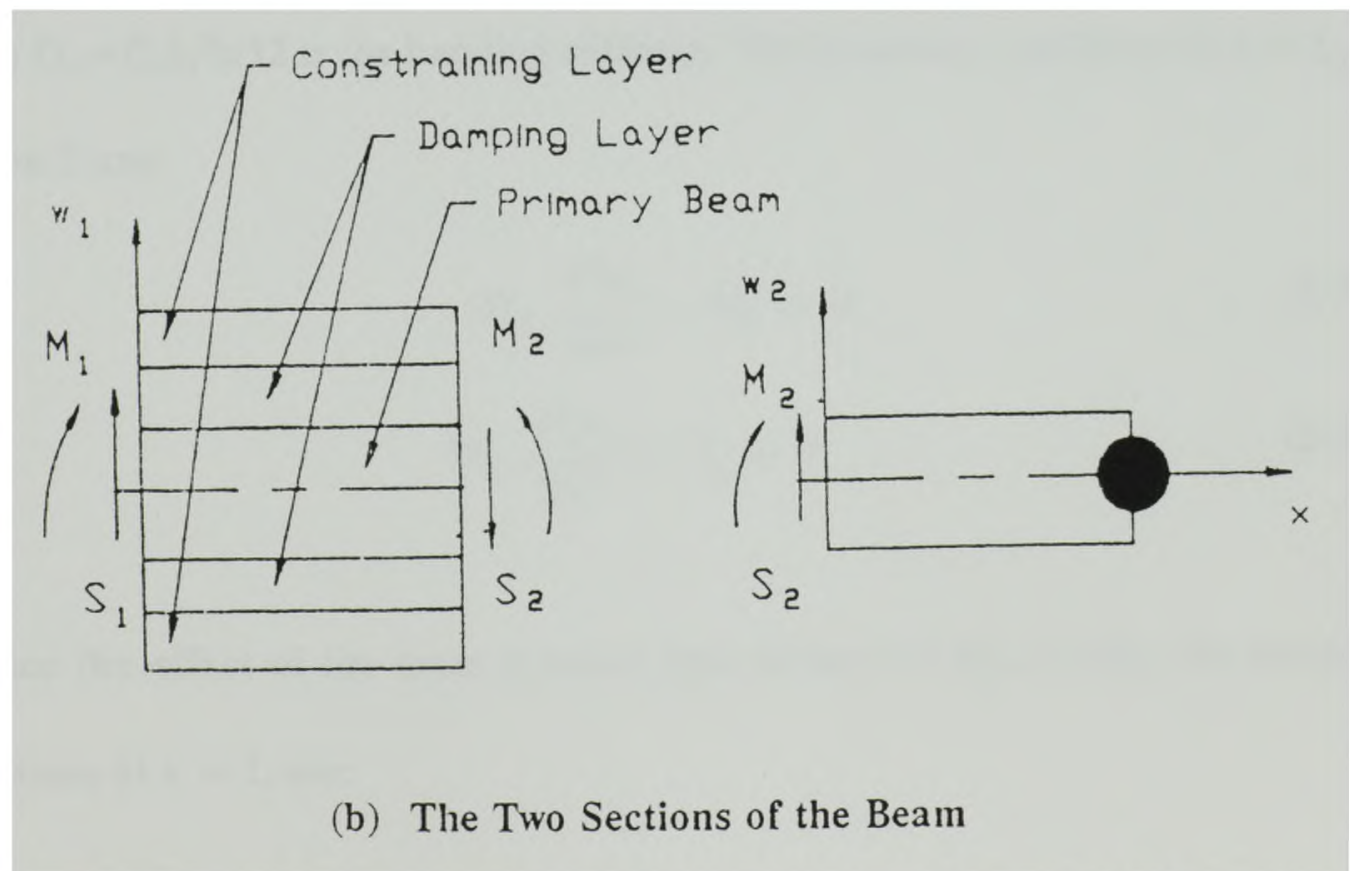

Figure 2.13 Model of the double sandwich cantilever beam with tip mass. 
The beam is separated into two sections. Section 1 is a double sandwich-type beam containing the damping and constraining layers, and Section 2 is the ordinary beam with mass $\mathrm{M}_{0}$ at its right-end. Matching conditions at the interface of the two sections will be applied on the transverse displacement, the rotation, and the shear force and moment continuity. The mass of the beam per unit length for Section 2 may be expressed as:

$$
\mathrm{m}_{20}=\mathrm{m}_{2}+\mathrm{M}_{0} \delta(\mathrm{x}-\mathrm{L}),
$$

where $\mathrm{m}_{2}$ is the mass per unit length of the uncovered portion of the beam, and $\delta(\mathrm{x}-\mathrm{L})$ is the Dirac delta function. Hence, the differential equation of motion for Section 2 is:

$$
D_{\mathrm{t}} \frac{\partial^{4} w_{2}}{\partial x^{4}}+\left[m_{2}+M_{0} \delta(x-L)\right] \frac{\partial^{2} w_{2}}{\partial t^{2}}=0,
$$

where $D_{t 1}=E_{1} h_{1}{ }^{3} b / 12$ is the bending stiffness. The boundary conditions at $x=L_{1}$ for Section 2 are:

$$
\begin{gathered}
D_{\mathrm{t} 1} \frac{\partial^{2} \mathrm{w}_{2}}{\partial \mathrm{x}^{2}}-\mathrm{M}_{2}=0 \\
\mathrm{D}_{\mathfrak{t} 1} \frac{\partial^{3} \mathrm{w}_{2}}{\partial \mathrm{x}^{3}}+\mathrm{S}_{2}=0
\end{gathered}
$$

Since the effect of the mass is taken into account in Eq. (2-38), the boundary conditions at $\mathrm{x}=\mathrm{L}$ are: 


$$
\begin{aligned}
& D_{\mathrm{t} 1} \frac{\partial^{2} w_{2}}{\partial x^{2}}=0 \\
& D_{\sharp 1} \frac{\partial^{3} w_{2}}{\partial x^{3}}=0 \text {. }
\end{aligned}
$$

The differential equations of motion for Section 1 are obtained by minimizing the variation in the total energy of the section. The equations are given in Levy and Chen (1994a) as:

$$
\left[\begin{array}{lll}
a_{11} & a_{12} & a_{13} \\
a_{21} & a_{22} & a_{23} \\
a_{31} & a_{32} & a_{33}
\end{array}\right]\left[\begin{array}{c}
w_{1} \\
\gamma_{c 1} \\
\gamma_{c 2}
\end{array}\right]=\{0\}
$$

where the $a_{i j}$ 's are given as follows:

$$
\begin{aligned}
& a_{11}=m \frac{\partial^{2}}{\partial t^{2}}+\left(D+E_{1} A_{1} B_{7}^{2}+E_{3} A_{3} B_{4}^{2}+E_{4} A_{4} B_{5}^{2}\right) \frac{\partial^{4}}{\partial x^{4}} \\
& a_{12}=+\left(E_{1} A_{1} B_{2} B_{7}-E_{3} A_{3} B_{4} B_{8}+E_{4} A_{4} B_{2} B_{5}\right) h_{c 1} \frac{\partial^{3}}{\partial x^{3}} \\
& a_{13}=\left(-E_{1} A_{1} B_{3} B_{7}-E_{3} A_{3} B_{3} B_{4}+E_{4} A_{4} B_{5} B_{6}\right) h_{c 2} \frac{\partial^{3}}{\partial x^{3}} \\
& a_{21}=\left(E_{1} A_{1} B_{2} B_{7}-E_{3} A_{3} B_{4} B_{8}+E_{4} A_{4} B_{2} B_{5}\right) h_{c 1} \frac{\partial^{3}}{\partial x^{3}} \\
& a_{22}=\left(B_{2}^{2} E_{1} A_{1}+B_{8}^{2} E_{3} A_{3}+B_{2}^{2} E_{4} A_{4}\right) h_{c 1}^{2} \frac{\partial^{2}}{\partial x^{2}}-G_{c 1} A_{c 1} \\
& a_{23}=\left(-E_{1} A_{1} B_{2} B_{3}+E_{3} A_{3} B_{3} B_{8}+E_{4} A_{4} B_{2} B_{6}\right) h_{c 1} h_{c 2} \frac{\partial^{2}}{\partial x^{2}} \\
& a_{31}=\left(E_{4} A_{4} B_{5} B_{6}-E_{1} A_{1} B_{3} B_{7}-E_{3} A_{3} B_{3} B_{4}\right) h_{c 1} h_{c 2} \frac{\partial^{3}}{\partial x^{3}} \\
& a_{32}=\left(E_{3} A_{3} B_{3} B_{8}-E_{1} A_{1} B_{2} B_{3}+E_{4} A_{4} B_{2} B_{6}\right) h_{c 1} h_{c 2} \frac{\partial^{2}}{\partial x^{2}} \\
& a_{33}=\left(E_{1} A_{1} B_{3}^{2}+E_{3} A_{3} B_{3}^{2}+E_{4} A_{4} B_{6}^{2}\right) h_{c 2}{ }^{2} \frac{\partial^{2}}{\partial x^{2}}-G_{c 2} A_{c 2} .
\end{aligned}
$$




$$
\begin{aligned}
& \left(E_{3} A_{3} B_{4} B_{8}-E_{1} A_{1} B_{2} B_{7}-E_{4} A_{4} B_{2} B_{5}\right) h_{c 1} \frac{\partial^{2} w_{1}}{\partial x^{2}}-\left(E_{1} A_{1} B_{2}^{2}+E_{3} A_{3} B_{8}^{2}\right. \\
& \left.+E_{4} A_{4} B_{2}^{2}\right) h_{c 1} \frac{\partial \gamma_{c l}}{\partial x}+\left(E_{1} A_{1} B_{2} B_{3}-E_{3} A_{3} B_{3} B_{8}\right. \\
& \left.-\mathrm{E}_{4} \mathrm{~A}_{4} \mathrm{~B}_{2} \mathrm{~B}_{6}\right) \mathrm{h}_{\mathrm{c} 1} \mathrm{~h}_{\mathrm{c} 2} \frac{\partial \gamma_{\mathrm{c} 2}}{\partial \mathrm{x}}=0 \\
& \left(E_{1} A_{1} B_{3} B_{7}+E_{3} A_{3} B_{3} B_{4}-E_{4} A_{4} B_{5} B_{6}\right) h_{c 2} \frac{\partial^{2} w_{1}}{\partial x^{2}}+\left(E_{1} A_{1} B_{2} B_{3}-E_{3} A_{3} B_{3} B_{8}\right. \\
& \left.-E_{4} A_{4} B_{2} B_{6}\right) h_{c 1} h_{c 2} \frac{\partial \gamma_{c 1}}{\partial x}-\left(E_{1} A_{1} B_{3}^{2}+E_{3} A_{3} B_{3}^{2}\right. \\
& \left.+E_{4} A_{4} B_{6}^{2}\right) h_{c 2}^{2} \frac{\partial \gamma_{c 2}}{\partial x}=0
\end{aligned}
$$

Here, $\mathrm{w}_{1}$ represents the displacement in Section 1 , and the remaining symbols used in Equations (2-40)-(2-41) are defined as follows:

$$
\begin{aligned}
& B_{1}=\left(E_{3} A_{3}+E_{4} A_{4}\right) / E A \\
& B_{2}=E_{3} A_{3} / E A \\
& B_{3}=E_{4} A_{4} / E A \\
& \left.B_{4}=\left[\left(E_{1} A_{1}+E_{4} A_{4}\right) h_{02}-E_{1} A_{1} h_{01}\right)\right] \\
& B_{5}=\left(E_{1} A_{1} h_{01}+E_{3} A_{3} h_{02}\right) / E A \\
& B_{6}=\left(E_{1} A_{1}+E_{3} A_{3}\right) / E A \\
& B_{7}=B_{1} h_{01}-B_{2} h_{02} \\
& B_{8}=\left(E_{1} A_{1}+E_{4} A_{4}\right) / E A \\
& D=b\left[E_{1} h_{1}^{3}+E_{3} h_{3}^{3}+E_{4} h_{4}^{3}\right] / 12 \\
& E A=E_{1} A_{1}+E_{3} A_{3}+E_{4} A_{4}
\end{aligned}
$$


The boundary conditions at $\mathrm{x}=0$ are:

$$
\begin{aligned}
& \mathrm{w}_{1}=0 \\
& \frac{\partial \mathrm{w}_{1}}{\partial \mathrm{x}}=0 \\
& \gamma_{\mathrm{c} 1}=0 \\
& \gamma_{\mathrm{c} 2}=0
\end{aligned}
$$

At $\mathrm{x}=\mathrm{L}_{1}$, the interface between damped and undamped parts of the beam, geometric continuity and generalized force continuity must apply, namely:

$$
\begin{aligned}
w_{1} & =w_{2} \\
\frac{\partial w_{1}}{\partial x} & =\frac{\partial w_{2}}{\partial x}
\end{aligned}
$$

Also, by using Equations (2-39a),(2-39b), (2-41), and (2-43), we obtain the conditions

$$
\begin{aligned}
& \text { at } \mathrm{x}=\mathrm{L}_{1} \\
& \left(D+E_{1} A_{1} B_{7}^{2}+E_{3} A_{3} B_{4}^{2}+E_{4} A_{4} B_{5}^{2}\right) \frac{\partial^{2} w_{1}}{\partial x^{2}}+\left(E_{1} A_{1} B_{2} B_{7}\right. \\
& \left.-E_{3} A_{3} B_{4} B_{8}+E_{4} A_{4} B_{2} B_{5}\right) h_{c l} \frac{\partial \gamma_{c l}}{\partial x}+\left(E_{4} A_{4} B_{5} B_{6}-E_{1} A_{1} B_{3} B_{7}\right. \\
& \left.-\mathrm{E}_{3} \mathrm{~A}_{3} \mathrm{~B}_{3} \mathrm{~B}_{4}\right) \mathrm{h}_{\mathrm{c} 2} \frac{\partial \gamma_{\mathrm{c} 2}}{\partial \mathrm{x}}+\mathrm{D}_{\mathrm{t}_{1}} \frac{\partial^{2} \mathrm{w}_{2}}{\partial \mathrm{x}^{2}}=0 \\
& \left(D+E_{1} A_{1} B_{7}^{2}+E_{3} A_{3} B_{4}^{2}+E_{4} A_{4} B_{5}^{2}\right) \frac{\partial^{3} w_{1}}{\partial x^{3}}+\left(E_{1} A_{1} B_{2} B_{7}-E_{3} A_{3} B_{4} B_{8}\right. \\
& \left.+\mathrm{E}_{4} \mathrm{~A}_{4} \mathrm{~B}_{2} \mathrm{~B}_{5}\right) \mathrm{h}_{\mathrm{cl}} \frac{\partial^{2} \gamma_{\mathrm{cl}}}{\partial \mathrm{x}^{2}}+\left(\mathrm{E}_{4} \mathrm{~A}_{4} \mathrm{~B}_{5} \mathrm{~B}_{6}-\mathrm{E}_{1} \mathrm{~A}_{1} \mathrm{~B}_{3} \mathrm{~B}_{7}\right. \\
& \left.-E_{3} A_{3} B_{3} B_{4}\right) h_{c 2} \frac{\partial^{2} \gamma_{c 2}}{\partial x^{2}}+D_{t_{1}} \frac{\partial^{3} w_{2}}{\partial x^{3}}=0 \\
& \left(E_{3} A_{3} B_{4} B_{8}-E_{1} A_{1} B_{2} B_{7}-E_{4} A_{4} B_{2} B_{5}\right) h_{c 1} \frac{\partial^{2} w_{1}}{\partial x^{2}}-\left(E_{1} A_{1} B_{2}^{2}+E_{3} A_{3} B_{8}^{2}\right.
\end{aligned}
$$




$$
\begin{gathered}
\left.+E_{4} A_{4} B_{2}^{2}\right) h_{c 1}{ }^{2} \frac{\partial \gamma_{c 1}}{\partial x}+\left(E_{1} A_{1} B_{2} B_{3}-E_{3} A_{3} B_{3} B_{8}\right. \\
\left.-E_{4} A_{4} B_{2} B_{6}\right) h_{c 1} h_{c 2} \frac{\partial \gamma_{c 2}}{\partial x}=0 \\
\left(E_{1} A_{1} B_{3} B_{7}+E_{3} A_{3} B_{3} B_{4}-E_{4} A_{4} B_{5} B_{6}\right) h_{c 2} \frac{\partial^{2} w_{1}}{\partial x^{2}}+\left(E_{1} A_{1} B_{2} B_{3}-E_{3} A_{3} B_{3} B_{8}\right. \\
\left.-E_{4} A_{4} B_{2} B_{6}\right) h_{c 1} h_{c 2} \frac{\partial \gamma_{c 1}}{\partial x}-\left(E_{1} A_{1} B_{3}^{2}+E_{3} A_{3} B_{3}^{2}\right. \\
\left.+E_{4} A_{4} B_{6}^{2}\right) h_{c 2} \frac{\partial \gamma_{c 2}}{\partial x}=0
\end{gathered}
$$

\subsubsection{Resonant frequency and loss factor}

As part of the design process of a partially covered, double sandwich-type cantilever beam, one will normally be interested in the resonant frequencies and loss factors in the first few modes of vibrations Rao (1978). To obtain the resonant frequencies and loss factors, assume the displacement solution of Section 2 to be in the form:

$$
w_{2}(x, t)=W_{2}(x) e^{j \Omega \cdot t}
$$

For mass $\mathrm{M}_{0}$ attached at $\mathrm{x}=\xi$, substituting Eq. (2-45) into Eq. (2-38) gives:

$$
\frac{\mathrm{d}^{4} \mathrm{~W}_{2}(\mathrm{x})}{\mathrm{dx}^{4}}-\frac{\omega^{4}}{\mathrm{~L}_{2}^{4}}\left[1+\frac{\mathrm{M}_{0}}{\mathrm{~m}_{0}} \mathrm{~L}_{2} \delta(\mathrm{x}-\xi)\right] \mathrm{W}_{2}(\mathrm{x})=0,
$$

where $\mathrm{m}_{0}=\mathrm{m}_{2} \mathrm{~L}_{2}, \mathrm{~L}_{2}=\mathrm{L}-\mathrm{L}_{1}, \mathrm{c}_{0}{ }^{4}=\mathrm{D}_{\mathrm{t} 1} / \mathrm{m}_{2}$, and $\omega^{2}=\Omega^{*} \mathrm{~L}_{2}{ }^{2} / \mathrm{c}_{0}{ }^{2}$. Taking the Laplace transform of Eq. (2-46) with respect to the variable $\mathrm{x}$ yields: 


$$
\begin{aligned}
& \mathrm{W}_{2}(\mathrm{~s})=\left[\mathrm{s}^{3} \mathrm{~W}_{2}\left(\mathrm{~L}_{1}\right)+\mathrm{s}^{2} \mathrm{~W}_{2}^{\prime}\left(\mathrm{L}_{1}\right)+\mathrm{sW}_{2}^{\prime \prime}\left(\mathrm{L}_{1}\right)+\mathrm{W}_{2}^{\prime \prime \prime}\left(\mathrm{L}_{1}\right)\right. \\
&\left.+\frac{\omega^{4}}{\mathrm{~L}_{2}^{3}} \frac{\mathrm{M}_{0}}{\mathrm{~m}_{0}} \mathrm{e}^{-\xi \mathrm{s}} \mathrm{W}_{2}(\xi)\right] /\left(\mathrm{s}^{4}-\frac{\omega^{4}}{\mathrm{~L}_{2}^{4}}\right),
\end{aligned}
$$

where s represents the transform variable. Taking the inverse transform of Eq. (247), we obtain:

$$
\begin{gathered}
\mathrm{W}_{2}(\mathrm{x})=\mathrm{W}_{2}(\xi)\left\{\hat{\mathrm{W}}_{2}\left(\mathrm{~L}_{1}\right) \mathrm{U}\left(\frac{\omega \mathrm{x}}{\mathrm{L}_{2}}\right)+\hat{\mathrm{W}}_{2}^{\prime}\left(\mathrm{L}_{1}\right)\left(\frac{\mathrm{L}_{2}}{\omega}\right) \mathrm{V}\left(\frac{\omega \mathrm{x}}{\mathrm{L}_{2}}\right)\right. \\
+\hat{\mathrm{W}}_{2}^{\prime \prime}\left(\mathrm{L}_{1}\right)\left(\frac{\mathrm{L}_{2}}{\omega}\right)^{2} \mathrm{~S}\left(\frac{\omega \mathrm{x}}{\mathrm{L}_{2}}\right)+\hat{\mathrm{W}}_{2}^{\prime \prime \prime}\left(\mathrm{L}_{1}\right)\left(\frac{\mathrm{L}_{2}}{\omega}\right)^{3} \mathrm{~T}\left(\frac{\omega \mathrm{x}}{\mathrm{L}_{2}}\right) \\
\left.+\frac{\mathrm{M}_{0}}{\mathrm{~m}_{0}} \omega \mathrm{T}\left[\frac{\omega(\mathrm{x}-\xi)}{\mathrm{L}_{2}}\right] \mathrm{H}(\mathrm{x}-\xi)\right\},
\end{gathered}
$$

where

$$
\begin{aligned}
& \hat{\mathrm{W}}_{2}^{(\mathrm{j})}\left(\mathrm{L}_{1}\right)=\mathrm{W}_{2}^{(\mathrm{j})}\left(\mathrm{L}_{1}\right) / \mathrm{W}_{2}(\xi), \\
& \mathrm{U}(\mathrm{x})=\frac{1}{2}[\cos (\mathrm{x})+\cosh (\mathrm{x})] \\
& \mathrm{V}(\mathrm{x})=\frac{1}{2}[\sin (\mathrm{x})+\sinh (\mathrm{x})], \\
& \mathrm{S}(\mathrm{x})=\frac{1}{2}[\cosh (\mathrm{x})-\cos (\mathrm{x})], \\
& \mathrm{T}(\mathrm{x})=\frac{1}{2}[\sinh (\mathrm{x})-\sin (\mathrm{x})],
\end{aligned}
$$


and $H(x)$ is the Heaviside step function. Since the solution must hold at $x=\xi$, Eq.(2-48) yields the following form of the frequency equation:

$$
\begin{aligned}
& \hat{\mathrm{W}}_{2}\left(\mathrm{~L}_{1}\right) \mathrm{U}\left(\frac{\omega_{\mathrm{n}} \xi}{\mathrm{L}_{2}}\right)+\hat{\mathrm{W}}_{2}^{\prime}\left(\mathrm{L}_{1}\right)\left(\frac{\mathrm{L}_{2}}{\omega_{\mathrm{n}}}\right) \mathrm{V}\left(\frac{\omega_{\mathrm{n}} \xi}{\mathrm{L}_{2}}\right)+ \\
& \hat{\mathrm{W}}_{2}^{\prime \prime}\left(\mathrm{L}_{1}\right)\left(\frac{\mathrm{L}_{2}}{\omega_{\mathrm{n}}}\right)^{2} \mathrm{~S}\left(\frac{\omega_{\mathrm{n}} \xi}{\mathrm{L}_{2}}\right)+\hat{\mathrm{W}}_{2}^{\prime \prime \prime}\left(\mathrm{L}_{1}\right)\left(\frac{\mathrm{L}_{2}}{\omega_{\mathrm{n}}}\right)^{3} \mathrm{~T}\left(\frac{\omega_{\mathrm{n}} \xi}{\mathrm{L}_{2}}\right)=1
\end{aligned}
$$

The corresponding eigenfunction is:

$$
\begin{aligned}
& \mathrm{W}_{2 \mathrm{n}}(\mathrm{x})=\hat{\mathrm{W}}_{2 \mathrm{n}}\left(\mathrm{L}_{1}\right) \mathrm{U}\left(\frac{\omega_{\mathrm{n}} \mathrm{x}}{\mathrm{L}_{2}}\right)+\hat{\mathrm{W}}_{2 \mathrm{a}}{ }^{\prime}\left(\mathrm{L}_{1}\right)\left(\frac{\mathrm{L}_{2}}{\omega_{\mathrm{n}}}\right) \mathrm{V}\left(\frac{\omega_{\mathrm{n}} \mathrm{x}}{\mathrm{L}_{2}}\right) \\
& +\hat{\mathrm{W}}_{2 \mathrm{n}}^{\prime \prime}\left(\mathrm{L}_{1}\right)\left(\frac{\mathrm{L}_{2}}{\omega_{\mathrm{n}}}\right)^{2} \mathrm{~S}\left(\frac{\omega_{\mathrm{n}} \mathrm{x}}{\mathrm{L}_{2}}\right)+\hat{\mathrm{W}}_{2 \mathrm{n}}^{\prime \prime \prime}\left(\mathrm{L}_{1}\right)\left(\frac{\mathrm{L}_{2}}{\omega_{\mathrm{n}}}\right)^{3} \mathrm{~T}\left(\frac{\omega_{\mathrm{n}} \mathrm{x}}{\mathrm{L}_{2}}\right) \\
& +\frac{\mathrm{M}_{0}}{\mathrm{~m}_{0}} \omega_{\mathrm{n}} \mathrm{T}\left[\frac{\omega_{\mathrm{n}}(\mathrm{x}-\xi)}{\mathrm{L}_{2}}\right] \mathrm{H}(\mathrm{x}-\xi),
\end{aligned}
$$

where $\hat{W}_{2 \mathrm{D}}\left(\mathrm{L}_{1}\right), \hat{\mathrm{W}}_{2 \mathrm{n}}{ }^{\prime}\left(\mathrm{L}_{1}\right), \hat{\mathrm{W}}_{2 \mathrm{n}}{ }^{\prime \prime}\left(\mathrm{L}_{1}\right)$, and $\hat{\mathrm{W}}_{2 \mathrm{n}}{ }^{\prime \prime \prime}\left(\mathrm{L}_{1}\right)$ will be determined from the boundary conditions. To ob tain these values, we need to look at Section 1. As with the displacement, $w_{2}$, we will assume a separable solution for $w_{1}$. The spatial displacement and shear strain functions in the first section of the beam as functions of the coordinate are assumed in the following form: 


$$
\begin{aligned}
& \bar{w}_{1 \mathrm{n}}(\mathrm{x})=\sum_{\mathrm{i}=1}^{8} \overline{\mathrm{A}}_{\mathrm{ni}} \mathrm{e}^{\mathrm{k} \cdot \mathrm{x}} \\
& \gamma_{\mathrm{cln}}(\mathrm{x})=\sum_{\mathrm{i}=1}^{8} \mathrm{f}_{\mathrm{ni}} \overline{\mathrm{A}}_{\mathrm{ni}} \mathrm{e}^{\mathrm{k}_{\mathrm{i}} \cdot \mathrm{x}} \\
& \gamma_{\mathrm{c} 2 \mathrm{n}}(\mathrm{x})=\sum_{\mathrm{i}=1}^{8} \mathrm{~g}_{\mathrm{ni}} \bar{A}_{\mathrm{ni}} \mathrm{e}^{\mathrm{k} \cdot \mathrm{x}}
\end{aligned}
$$

where

$$
\begin{aligned}
& f_{n i}=\frac{d_{23} d_{31}-d_{21} d_{33}}{d_{22} d_{33}-d_{32} d_{23}} \\
& g_{n i}=\frac{d_{21} d_{32}-d_{22} d_{31}}{d_{22} d_{33}-d_{32} d_{23}} \\
& d_{11}=-m \Omega_{n}^{* 2}+\left(D+E_{1} A_{1} B_{7}^{2}+E_{3} A_{3} B_{4}{ }^{2}+E_{4} A_{4} B_{5}{ }^{2}\right) k_{n i}^{* 4} \\
& d_{12}=+\left(E_{1} A_{1} B_{2} B_{7}-E_{3} A_{3} B_{4} B_{8}+E_{4} A_{4} B_{2} B_{5}\right) h_{c 1} k_{n i}{ }^{* 3} \\
& d_{13}=\left(-E_{1} A_{1} B_{3} B_{7}-E_{3} A_{3} B_{3} B_{4}+E_{4} A_{4} B_{5} B_{6}\right) h_{c 2} k_{n i}^{* 3} \\
& d_{21}=\left(E_{1} A_{1} B_{2} B_{7}-E_{3} A_{3} B_{4} B_{8}+E_{4} A_{4} B_{2} B_{5}\right) h_{c 1} k_{n i}^{* 3} \\
& d_{22}=\left(B_{2}{ }^{2} E_{1} A_{1}+B_{8}{ }^{2} E_{3} A_{3}+B_{2}{ }^{2} E_{4} A_{4}\right) h_{c 1}{ }^{2} k_{n i}{ }^{* 2}-G_{c 1} A_{c 1} \\
& d_{23}=\left(-E_{1} A_{1} B_{2} B_{3}+E_{3} A_{3} B_{3} B_{8}+E_{4} A_{4} B_{2} B_{6}\right) h_{c 1} h_{c 2} k_{n i}{ }^{* 2} \\
& d_{31}=\left(E_{4} A_{4} B_{5} B_{6}-E_{1} A_{1} B_{3} B_{7}-E_{3} A_{3} B_{3} B_{4}\right) h_{c 2} k_{n i}^{* 3} \\
& d_{32}=\left(E_{3} A_{3} B_{3} B_{8}-E_{1} A_{1} B_{2} B_{3}+E_{4} A_{4} B_{2} B_{6}\right) h_{c 1} h_{c 2} k_{n i}^{* 2} \\
& d_{33}=\left(E_{1} A_{1} B_{3}{ }^{2}+E_{3} A_{3} B_{3}{ }^{2}+E_{4} A_{4} B_{6}{ }^{2}\right) h_{c 2}{ }^{2} k_{n i}{ }^{2}-G_{c 2} A_{c 2}
\end{aligned}
$$


Using the same methodology defined in Section 2.1.3, the characteristic equation for section 1 is expressed as:

$$
\operatorname{det}\left[\begin{array}{lll}
\bar{a}_{11} & \bar{a}_{12} & \bar{a}_{13} \\
\bar{a}_{21} & \bar{a}_{22} & \bar{a}_{23} \\
\bar{a}_{31} & \bar{a}_{32} & \bar{a}_{33}
\end{array}\right]=0
$$

where

$$
\begin{aligned}
& \bar{a}_{11}=-m \Omega_{\mathrm{n}}{ }^{2}+\left(D+E_{1} A_{1} B_{7}{ }^{2}+E_{3} A_{3} B_{4}{ }^{2}+E_{4} A_{4} B_{5}{ }^{2}\right) k_{n}{ }^{* 4} \\
& \bar{a}_{12}=+\left(E_{1} A_{1} B_{2} B_{7}-E_{3} A_{3} B_{4} B_{8}+E_{4} A_{4} B_{2} B_{5}\right) h_{c l} k_{n}^{* 3} \\
& \bar{a}_{13}=\left(-E_{1} A_{1} B_{3} B_{7}-E_{3} A_{3} B_{3} B_{4}+E_{4} A_{4} B_{5} B_{6}\right) h_{c 2} k_{n}{ }^{* 3} \\
& \bar{a}_{21}=\left(E_{1} A_{1} B_{2} B_{7}-E_{3} A_{3} B_{4} B_{8}+E_{4} A_{4} B_{2} B_{5}\right) h_{c 1} k_{n}^{* 3} \\
& \bar{a}_{22}=\left(B_{2}{ }^{2} E_{1} A_{1}+B_{8}{ }^{2} E_{3} A_{3}+B_{2}{ }^{2} E_{4} A_{4}\right) h_{c 1}{ }^{2} k_{n}{ }^{* 2}-G_{c 1} A_{c 1} \\
& \bar{a}_{23}=\left(-E_{1} A_{1} B_{2} B_{3}+E_{3} A_{3} B_{3} B_{8}+E_{4} A_{4} B_{2} B_{6}\right) h_{c 1} h_{c 2} k_{n}{ }^{* 2} \\
& \bar{a}_{31}=\left(E_{4} A_{4} B_{5} B_{6}-E_{1} A_{1} B_{3} B_{7}-E_{3} A_{3} B_{3} B_{4}\right) h_{c 2} k_{n}^{* 3} \\
& \bar{a}_{32}=\left(E_{3} A_{3} B_{3} B_{8}-E_{1} A_{1} B_{2} B_{3}+E_{4} A_{4} B_{2} B_{6}\right) h_{c 1} h_{c 2} k_{n}^{* 2} \\
& \bar{a}_{33}=\left(E_{1} A_{1} B_{3}{ }^{2}+E_{3} A_{3} B_{3}{ }^{2}+E_{4} A_{4} B_{6}{ }^{2}\right) h_{c 2}{ }^{2} k_{n}{ }^{* 2}-G_{c 2} A_{c 2} .
\end{aligned}
$$

In the same manner as described in Section 2.1 .3 , the various boundary conditions and continuity conditions may be expressed as:

$$
\text { at } \mathbf{x}=0 \text { : }
$$

$$
\sum_{i=1}^{8} \bar{A}_{n i} k_{n i}^{*}=0
$$




$$
\begin{gathered}
\sum_{i=1}^{n} \bar{A}_{n i}=0 \\
\sum_{i=1}^{8} f_{n i} \bar{A}_{n i}=0 \\
\sum_{i=1}^{8} g_{n i} \bar{A}_{n i}=0
\end{gathered}
$$

at $\mathbf{x}=\mathbf{L}_{1}$ :

The moment balance at the interface, as viewed from Section 1 (constrained part), leads to the following:

$$
\begin{aligned}
& \sum_{i=1}^{8}\left(D+E_{1} A_{1} B_{7}^{2}+E_{3} A_{3} B_{4}^{2}+E_{4} A_{4} B_{5}^{2}\right) \bar{A}_{n i} k_{n i}^{* 2} e^{k_{n i}^{*} L_{1}} \\
+ & \sum_{i=1}^{8}\left(E_{1} A_{1} B_{2} B_{7}-E_{3} A_{3} B_{4} B_{8}+E_{4} A_{4} B_{2} B_{5}\right) h_{c 1} f_{n i} A_{n i} k_{n i}^{*} e^{k_{n i}^{*} L_{1}} \\
+ & \sum_{i=1}^{8}\left(E_{4} A_{4} B_{5} B_{6}-E_{1} A_{1} B_{3} B_{7}-E_{3} A_{3} B_{3} B_{4}\right) h_{c 2} g_{n i} \bar{A}_{n i} k_{n i}^{*} e^{k_{n i}^{*} L_{1}}+M_{2}=0
\end{aligned}
$$

The moment balance at the interface, as viewed from Section 2 (unconstrained part), leads to:

$$
\begin{aligned}
& \mathrm{D}_{\mathrm{t} 1}\left[\hat{\mathrm{W}}_{2 \mathrm{n}}\left(\mathrm{L}_{1}\right)\left(\frac{\omega_{\mathrm{n}}}{\mathrm{L}_{2}}\right)^{2} \mathrm{~S}\left(\frac{\omega_{\mathrm{n}} \mathrm{L}_{1}}{\mathrm{~L}_{2}}\right)+\hat{\mathrm{W}}_{2 \mathrm{n}}^{\prime}\left(\mathrm{L}_{1}\right)\left(\frac{\omega_{\mathrm{n}}}{\mathrm{L}_{2}}\right) \mathrm{T}\left(\frac{\omega_{\mathrm{n}} \mathrm{L}_{1}}{\mathrm{~L}_{2}}\right)\right. \\
& \left.+\hat{\mathrm{W}}_{2 \mathrm{n}}^{\prime \prime}\left(\mathrm{L}_{1}\right) \mathrm{U}\left(\frac{\omega_{\mathrm{n}} \mathrm{L}_{1}}{\mathrm{~L}_{2}}\right)+\hat{\mathrm{W}}_{2 \mathrm{n}}^{\prime \prime \prime}\left(\mathrm{L}_{1}\right)\left(\frac{\mathrm{L}_{2}}{\omega_{\mathrm{n}}}\right) \mathrm{V}\left(\frac{\omega_{\mathrm{n}} \mathrm{L}_{1}}{\mathrm{~L}_{2}}\right)\right]+\mathrm{M}_{2}=0
\end{aligned}
$$


The shear force balance at the interface, as viewed from Section 1, leads to the following:

$$
\begin{aligned}
& \sum_{i=1}^{8}\left(D+E_{1} A_{1} B_{7}^{2}+E_{3} A_{3} B_{4}^{2}+E_{4} A_{4} B_{5}^{2}\right) A_{n i} k_{n i}^{*} e^{k_{n i}^{*} L_{1}} \\
+ & \sum_{i=1}^{8}\left(E_{1} A_{1} B_{2} B_{7}-E_{3} A_{3} B_{4} B_{8}+E_{4} A_{4} B_{2} B_{5}\right) h_{c 1} f_{n i} \bar{A}_{\mathrm{ii}} k_{n i}^{* 2} e^{k_{n i}^{*} L_{1}} \\
+ & \sum_{i=1}^{8}\left(E_{4} A_{4} B_{5} B_{6}-E_{1} A_{1} B_{3} B_{7}-E_{3} A_{3} B_{3} B_{4}\right) h_{c 2} g_{0 i} A_{n i} k_{n i}^{* 2} e^{k_{n j}^{*} L_{1}-S_{2}}=0
\end{aligned}
$$

The shear force balance at the interface, as viewed from Section 2, leads to:

$$
\begin{aligned}
& \mathrm{D}_{\mathrm{n}}\left[\hat{\mathrm{W}}_{2 \mathrm{n}}\left(\mathrm{L}_{1}\right)\left(\frac{\omega_{\mathrm{n}}}{\mathrm{L}_{2}}\right)^{3} \mathrm{~V}\left(\frac{\omega_{\mathrm{n}} \mathrm{L}_{1}}{\mathrm{~L}_{2}}\right)+\hat{\mathrm{W}}_{2 \mathrm{n}}^{\prime}\left(\mathrm{L}_{1}\right)\left(\frac{\omega_{\mathrm{n}}}{\mathrm{L}_{2}}\right)^{2} \mathrm{~S}\left(\frac{\omega_{\mathrm{n}} \mathrm{L}_{1}}{\mathrm{~L}_{2}}\right)\right. \\
& \left.+\hat{\mathrm{W}}_{2 \mathrm{n}}^{\prime \prime}\left(\mathrm{L}_{1}\right)\left(\frac{\omega_{\mathrm{n}}}{\mathrm{L}_{2}}\right) \mathrm{T}\left(\frac{\omega_{\mathrm{n}} \mathrm{L}_{1}}{\mathrm{~L}_{2}}\right)+\hat{\mathrm{W}}_{2 \mathrm{n}}^{\prime \prime \prime}\left(\mathrm{L}_{1}\right) \mathrm{U}\left(\frac{\omega_{\mathrm{n}} \mathrm{L}_{1}}{\mathrm{~L}_{2}}\right)\right]+\mathrm{S}_{2}=0
\end{aligned}
$$

The equilibrium of the moment generated by the shear of each of the two damping layers is given by the following two equations:

$$
\begin{aligned}
& \sum_{i=1}^{8}\left(E_{3} A_{3} B_{4} B_{8}-E_{1} A_{1} B_{2} B_{7}-E_{4} A_{4} B_{2} B_{5}\right) h_{c 1} A_{n i} k_{n i}^{* 2} e^{k_{n i}^{*} L_{1}} \sum_{i=1}^{8}\left(B_{2}^{2} E_{1} A_{1}\right. \\
& \left.+E_{3} A_{3} B_{8}^{2}+E_{4} A_{4} B_{2}^{2}\right) h_{c i}^{2} f_{n i} \bar{A}_{1 i} k_{n i}^{*} e^{k_{n i}^{*} L_{1}} \\
& +\sum_{i=1}^{8}\left(E_{1} A_{1} B_{2} B_{3}-E_{3} A_{3} B_{3} B_{8}-E_{4} A_{4} B_{2} B_{6}\right) h_{c 1} h_{c 2} g_{n i} \bar{A}_{n i} k_{n i}^{*} e^{k_{n i}^{*} L_{1}}=0 \\
& \sum_{i=1}^{8}\left(E_{1} A_{1} B_{3} B_{7}+E_{3} A_{3} B_{3} B_{4}-E_{4} A_{4} B_{5} B_{6}\right) h_{c 2} \bar{A}_{n i} k_{n i}^{* 2} e^{k_{n i}^{*} L_{1}}-\sum_{i=1}^{8}\left(E_{1} A_{1} B_{2} B_{3}\right. \\
& \left.-E_{3} A_{3} B_{3} B_{8}+E_{4} A_{4} B_{2} B_{6}\right) h_{c 1} h_{c 2} f_{n i} \bar{A}_{n i} k_{n i}^{*} e^{k_{n i}^{*} L_{1}}-\sum_{i=1}^{8}\left(E_{1} A_{1} B_{3}^{2}+E_{3} A_{3} B_{3}^{2}\right.
\end{aligned}
$$




$$
\left.+E_{4} A_{4} B_{6}^{2}\right) h_{c 2}^{2} g_{n i} \bar{A}_{n i} k_{n i}^{*} e^{k_{n i}^{*} L_{1}}=0
$$

$$
\text { at } \mathbf{x}=\mathbf{L}:
$$

The zero moment and transverse force conditions become:

$$
\begin{aligned}
& \hat{W}_{2 \mathrm{n}}\left(\mathrm{L}_{1}\right)\left(\frac{\omega_{\mathrm{n}}}{\mathrm{L}_{2}}\right)^{2} \mathrm{~S}\left(\frac{\omega_{\mathrm{n}} \mathrm{L}}{\mathrm{L}_{2}}\right)+\hat{\mathrm{W}}_{2 \mathrm{n}}^{\prime}\left(\mathrm{L}_{1}\right)\left(\frac{\omega_{\mathrm{n}}}{\mathrm{L}_{2}}\right) \mathrm{T}\left(\frac{\omega_{\mathrm{n}} \mathrm{L}}{\mathrm{L}_{2}}\right) \\
& +\hat{\mathrm{W}}_{2 \mathrm{n}}^{\prime \prime}\left(\mathrm{L}_{1}\right) \mathrm{U}\left(\frac{\omega_{\mathrm{n}} \mathrm{L}}{\mathrm{L}_{2}}\right)+\hat{\mathrm{W}}_{2 \mathrm{n}}^{\prime \prime \prime}\left(\mathrm{L}_{1}\right)\left(\frac{\mathrm{L}_{2}}{\omega_{\mathrm{n}} \mathrm{L}}\right) \mathrm{V}\left(\frac{\omega_{\mathrm{n}} \mathrm{L}}{\mathrm{L}_{2}}\right)=0 \\
& \hat{\mathrm{W}}_{2 \mathrm{n}}\left(\mathrm{L}_{1}\right)\left(\frac{\omega_{\mathrm{n}}}{\mathrm{L}_{2}}\right)^{3} \mathrm{~V}\left(\frac{\omega_{\mathrm{n}} \mathrm{L}}{\mathrm{L}_{2}}\right)+\hat{\mathrm{W}}_{2 \mathrm{n}}^{\prime}\left(\mathrm{L}_{1}\right)\left(\frac{\omega_{\mathrm{n}}}{\mathrm{L}_{2}}\right)^{2} \mathrm{~S}\left(\frac{\omega_{\mathrm{n}} \mathrm{L}}{\mathrm{L}_{2}}\right) \\
& +\hat{\mathrm{W}}_{2 \mathrm{n}}^{\prime \prime}\left(\mathrm{L}_{1}\right)\left(\frac{\omega_{\mathrm{n}}}{\mathrm{L}_{2}}\right) \mathrm{T}\left(\frac{\omega_{\mathrm{n}} \mathrm{L}}{\mathrm{L}_{2}}\right)+\hat{\mathrm{W}}_{2 \mathrm{n}}^{\prime \prime \prime}\left(\mathrm{L}_{1}\right) \mathrm{U}\left(\frac{\omega_{\mathrm{n}} \mathrm{L}}{\mathrm{L}_{2}}\right)+\frac{\mathrm{M}_{0}}{\mathrm{~m}_{0}} \frac{\omega_{\mathrm{n}}^{4}}{\mathrm{~L}_{2}^{2}}=0
\end{aligned}
$$

The other two supplementary conditions representing the equilibrium of the axial forces in the constraining layers are:

$$
\begin{aligned}
& E_{3} A_{3} \frac{\partial u_{3}}{\partial x}=0 \\
& E_{4} A_{4} \frac{\partial u_{4}}{\partial x}=0
\end{aligned}
$$

They may be written as:

$$
\sum_{i=1}^{8}\left(B_{4} \dot{k}_{n i}^{2} e^{k_{i} L_{4}}-B_{8} h_{c 1} f_{n i} \dot{k}_{n i}^{*} e^{k_{i}^{*} L_{1}}-B_{3} h_{c 2} g_{n i} \dot{k}_{n i} e^{k_{i}: L}\right)=0
$$




$$
\sum_{i=1}^{8}\left(B_{8} k_{n i}^{* 2} e^{k \cdot h_{1}}+B_{2} h_{c 1} f_{n i} \dot{k}_{n i} e^{k_{i} \cdot L_{1}}+B_{6} h_{c 2} g_{n i} \dot{k}_{n i} e^{k_{i j} \cdot L_{1}}\right)=0
$$

We can eliminate $M_{2}$ and $S_{2}$ from the above equations by combining Eqs (2-59), (2$60),(2-61)$ and (2-62). Hence, we have a total of 14 equations to determine the unknowns. In the above equations, if $\mathrm{M}_{0}=0$, then the equations reduce to the case of the double sandwich-type cantilever with no mass at the free end. Equations (249), (2-54) to (2-68) are nonlinear, complex valued equations for unknowns, $\Omega_{n}{ }^{*}$. A numerical scheme was used to obtain the results shown below. The uncovered cantilever beam with tip mass provided starting values for the scheme. These starting values and the secant method were employed to solve for $\Omega_{\mathrm{a}}{ }^{*}$. By assuming a complex frequency factor, $\mathrm{p}^{*}$, the real frequency factor (or the resonant frequency factor ), p, and the loss factor, $\eta$, of the beam are related to $\Omega_{\mathrm{a}}{ }^{*}$ by equation (3-32). 


\subsubsection{Numerical results}

Numerical results for the various mass ratios were obtained. The input parameters employed in the previously described numerical scheme were the material and geometrical properties, mass density, and core loss factor. The values chosen for the computation, unless stated otherwise, were:

$$
\begin{aligned}
& \mathrm{E}_{1}=\mathrm{E}_{3}=\mathrm{E}_{5}=20.6 \times 10^{10} \mathrm{~N} / \mathrm{m}^{2} ; \mathrm{G}_{\mathrm{cl}}=\mathrm{G}_{\mathrm{c} 2}=0.42 \times 10^{8} \mathrm{~N} / \mathrm{m}^{2} ; \mathrm{L}=0.5 \mathrm{~m}, \mathrm{~h}_{1}=0.02 \mathrm{~m} ; \\
& \rho_{1}=\rho_{3}=\rho_{4}=7850 \mathrm{Kg} / \mathrm{m}^{3} ; \rho_{\mathrm{cl}}=\rho_{\mathrm{c} 2}=3140 \mathrm{Kg} / \mathrm{m}^{3} ; \text { and } \mathrm{h}_{3}=\mathrm{h}_{4}=\mathrm{h}_{\mathrm{cl}}=\mathrm{h}_{\mathrm{c} 2}=1 / 2 \mathrm{~h}_{1} .
\end{aligned}
$$

\section{Mass Ratio Versus Resonant Frequency}

Figure 2.14 shows the lowest resonant frequency versus the mass ratio (the attached mass to the beam of the beam). It can be observed that the increase in mass ratio will decrease the value of the lowest resonant frequency of the beam. This occurs because the increase in end mass causes the system to execute larger periodic excursions, increasing the period of oscillation, and hence, decreasing the frequency of oscillation. Also, the increase in the damping and constraining layer length will increase the value of the resonant frequency of the beam at constant mass ratio. This latter effect is the same as that obtained in Figure 2.3. This occurs, in this case, because the constraining layer values of $E_{3}$ and $E_{4}$ are the same as $E_{1}$, and the shear moduli are much smaller in magnitude. This has the effect of 


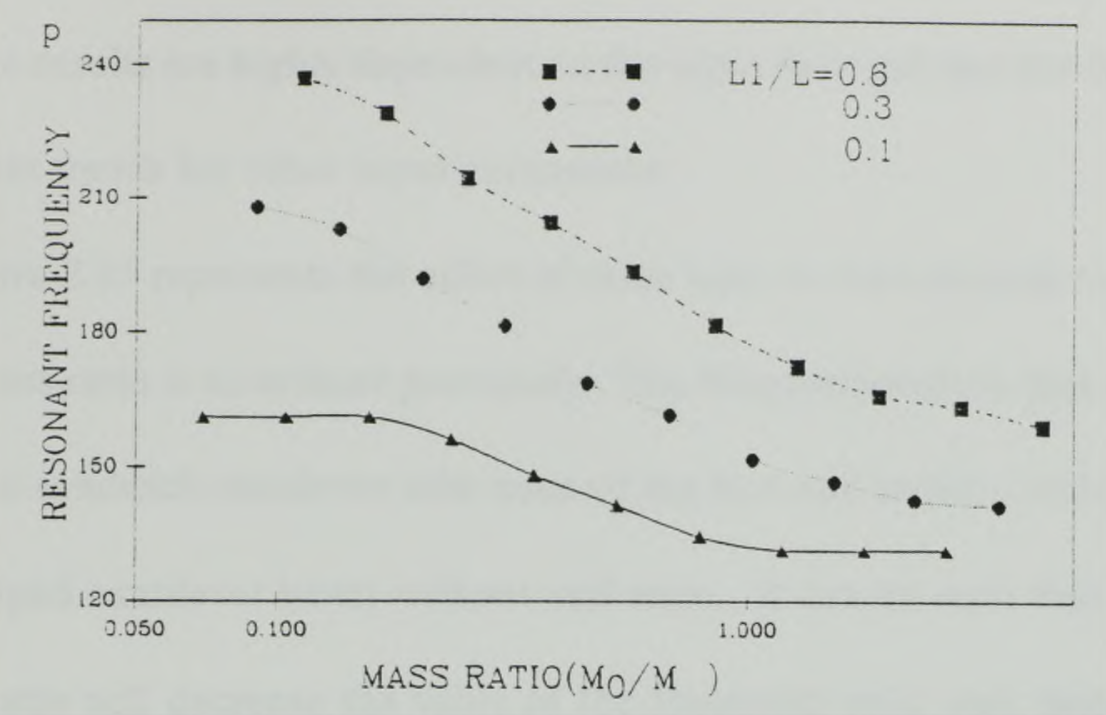

Figure 2.14 Resonant frequency versus mass ratio (the attached mass to the mass of the beam)

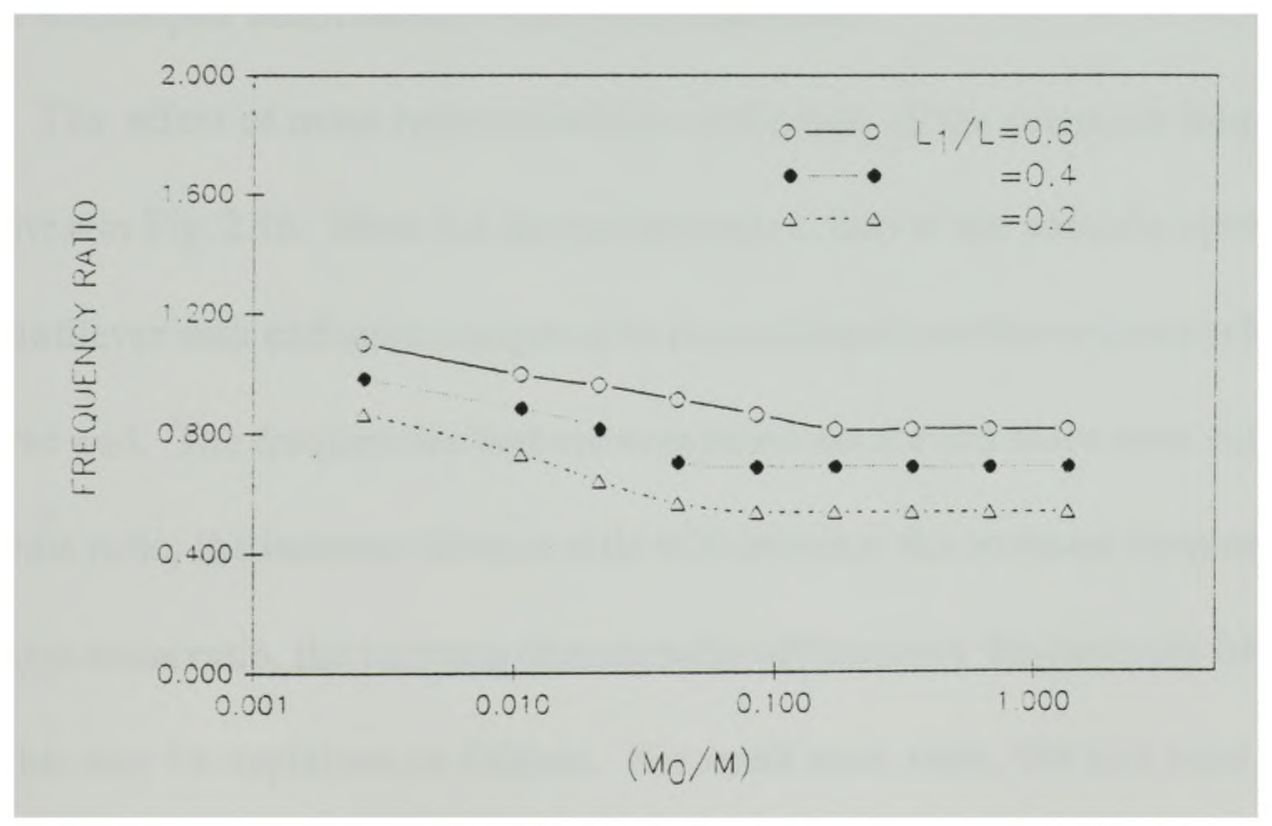

Figure 2.15 Frequency ratio versus mass ratio 
moving the cantilever end of the beam to $L_{1}$, thereby shortening the effective length of the beam. This, of course, increases the resonant frequency. It must be noted that the results are highly dependent on the input data and that the figures may have different trends for other input parameters.

Figure 2.15 represents the effect of mass ratio to the resonant frequency ratio. The mass ratio is as defined previously. The frequency ratio is that for the partially covered sandwich cantilever with mass of the free end to the resonant frequency of undamped cantilever beam without end mass. It can be seen that the increase in mass ratio will decrease the value of the frequency ratio and, hence the resonant frequency. This coincides with Fig. 2.14 because in this case the resonant frequency of undamped beam doesn't vary with end mass.

The effect of mass ratio to another definition of the resonant frequency ratio is given in Fig. 2.16. Here the frequency ratio is that of the partially covered sandwich cantilever with end mass compared to an undamped cantilever beam with mass at the free end. The frequencies that are compared are for the same mass ratio. For small mass ratio, the increase of mass ratio will decrease the resonant frequency ratio. For large mass ratio, the increase of mass ratio will increase the resonant frequency ratio. This may be explained as follows. For small mass ratio, the end mass plays a more important role to the damped beam than to the undamped beam, causing a decrease in the resonant frequency ratio. But for large mass ratio, even though the large end 
mass causes a larger resonant frequency, the sandwich structure dominates and acts as if the fixed end of the beam is moved to the right, thus increasing the resonant frequency ratio of the sandwich beam compared to the uncovered beam.

From Figures 2.15 and 2.16 we note that, under certain circumstances, the covered beam will have a lower resonant frequency when an end mass is added (i.e., when a robot arm picks up a remote mass in its end effector) than an uncovered beam without end mass (i.e., an undamped robot arm without a mass in its end effector, Fig. 2.15). Also, the addition of damping material can reduce the resonant frequency (Fig. $4, \mathrm{~L}_{1} / \mathrm{L}=0.6$ for $\mathrm{M}_{d} \mathrm{M}$ between 0.002 and 0.8 ) when compared to an uncovered beam having the same mass ratio. This indicates that large damping coverage may be counterproductive and one must be sensitive to this possibility.

Figure 2.17 represents the effect of mass ratio to the ratio of the second mode of resonant frequency. (The definition of frequency ratio in the same as in Fig. 2-16). It may be seen that the increase of mass ratio will increase the resonant frequency ratio, i.e., sandwich structure dominates, and that coverage length plays no distinguishing role part $\mathrm{M}_{\delta} \mathrm{M}=0.1$. From the three figures (Figures 2.15 to 2.17), it appears that $L_{1} / L$ should be less than 0.4 to get good use of the damping coverage.

\section{System Loss Factor Versus Damping Coverage}

Figure 2.18 represents the system loss factor versus the damping coverage. Here 


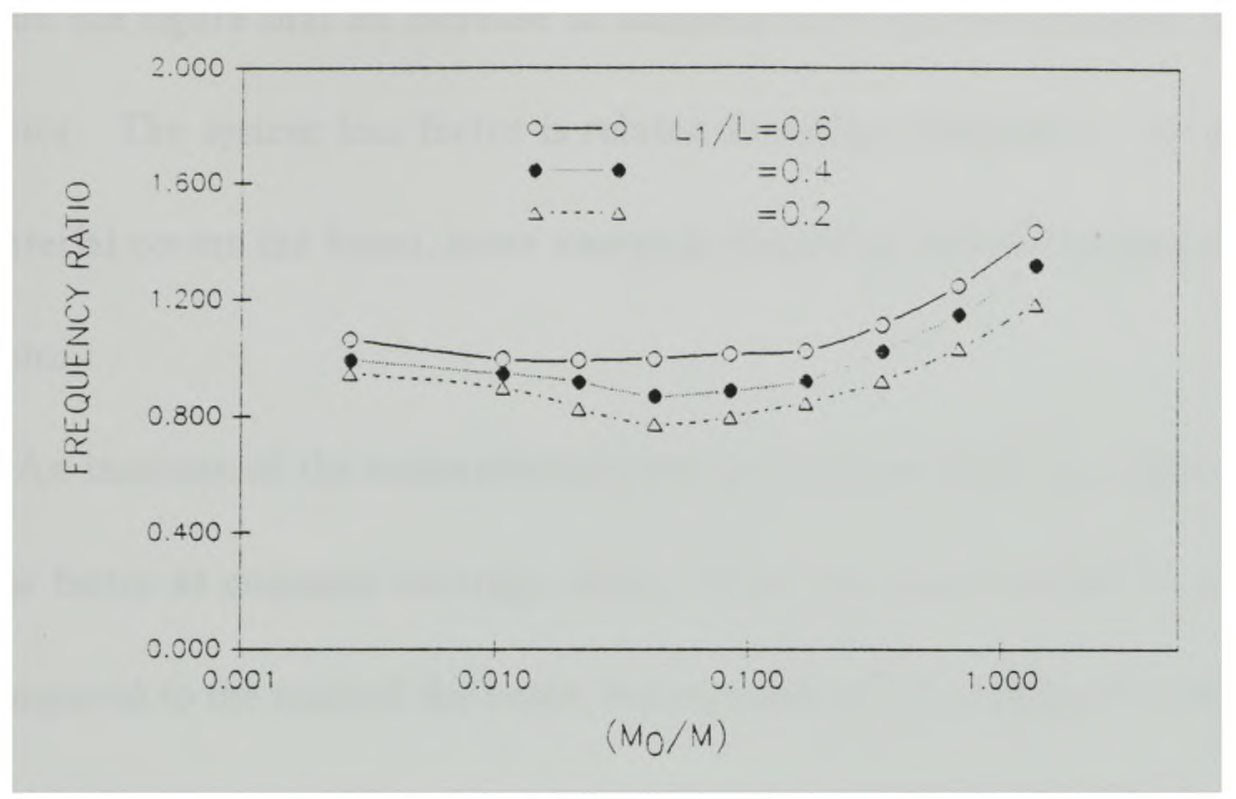

Figure 2.16 Frequency ratio versus mass ratio

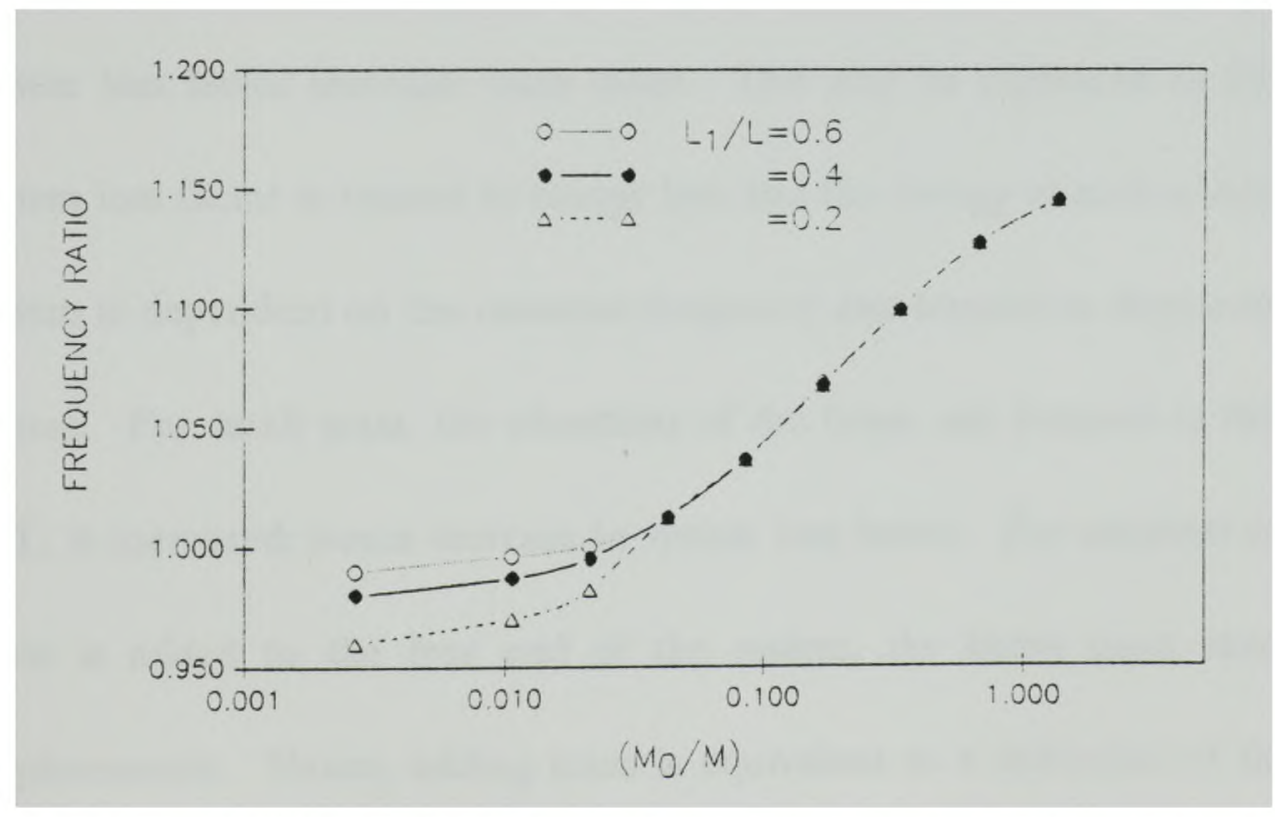

Figure 2.17 Second mode resonant frequency ratio versus mass ratio 
the core loss factor, $\eta_{\mathrm{cl}}$, is taken as 1 to simplify the calculations. It may be seen from the figure that an increase in damping coverage will increase the system loss factor. The system loss factor is related to energy dissipation. As more damping material covers the beam, more energy is dissipated, thus increasing the system loss factor.

An increase of the concentrated mass will also decrease the value of the system loss factor at constant coverage ratios. When the concentrated mass is very small compared to the mass of the beam, the increase of $L_{1} / L$ will increase the value of the system loss factor. When the concentrated mass is large, the increase of $L_{1} / L$ will decrease the value of the system loss factor. As more of the beam is covered the system loss factor increases once more. This may be explained as follows: The system loss factor is related to energy loss and the energy of such a viscoelastic system is dependent on the resonant frequency and transverse displacement of the system. For small mass, the vibrations of the beam are damped as the coverage, $\mathrm{L}_{1} / \mathrm{L}$, is increased; hence increase in system loss factor. For constant coverage, as mass is added to the free end of the system, the beam must execute larger displacements. Hence, adding mass is equivalent to a reduction of the damping effect of the coverage and decreasing energy loss - thus a lower system loss factor. After some point, additional damping coverage counters the effect of the increase 


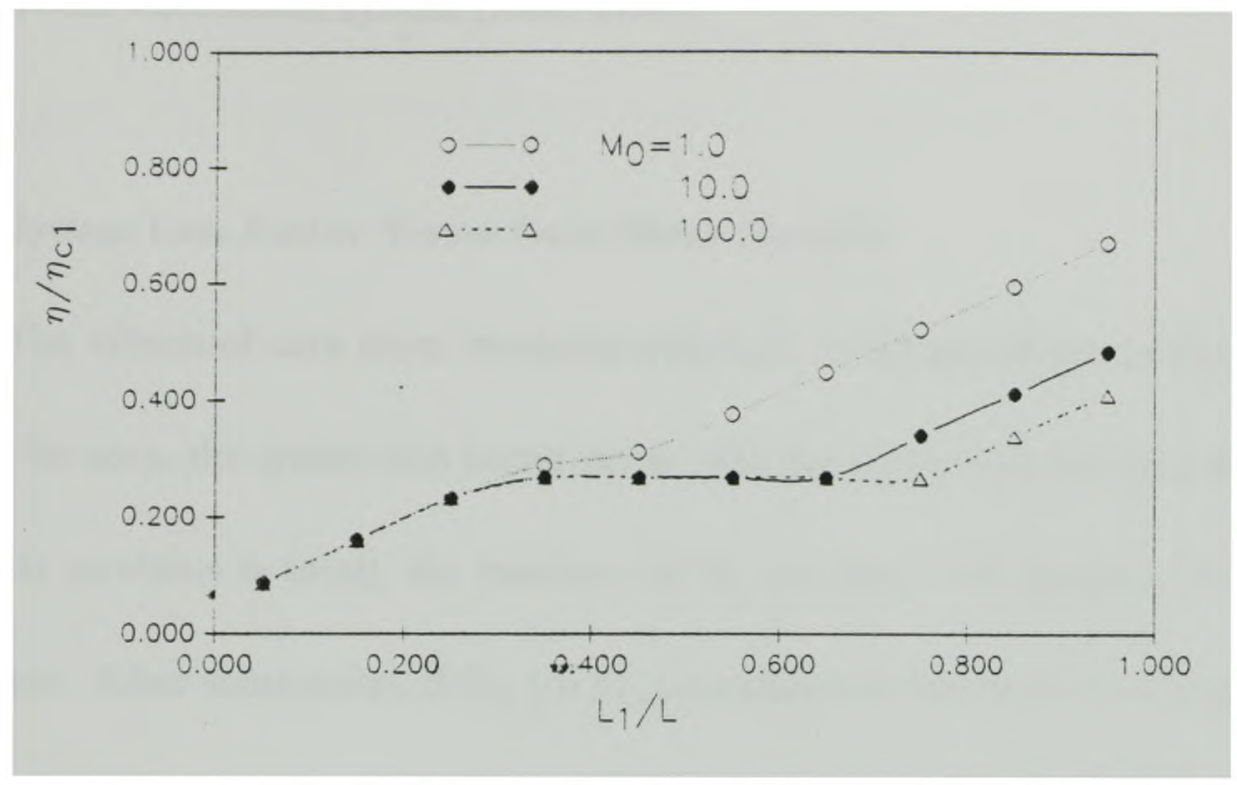

Figure 2.18 System loss factor versus coverage ratio

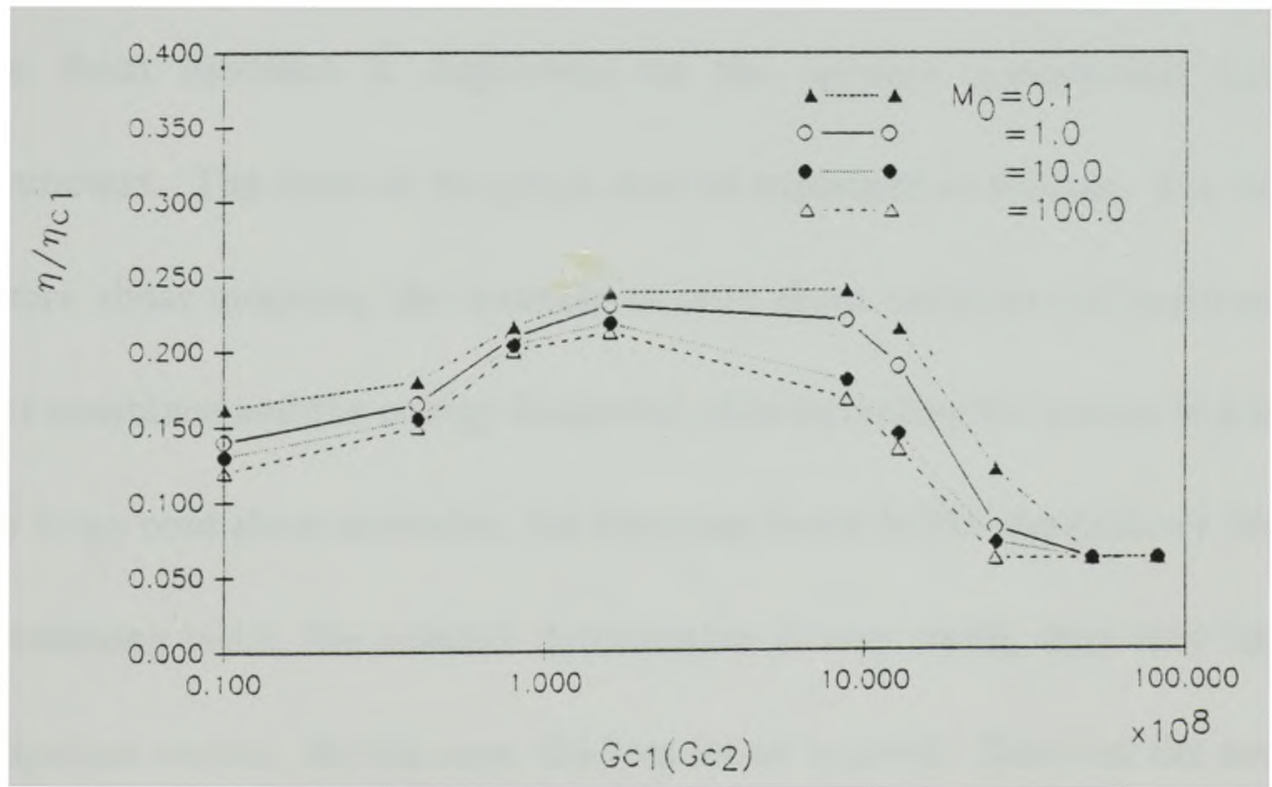

Figure 2.19 Loss factor versus shear modulus with $\mathrm{L}_{1} / \mathrm{L}=0.2$ 
in mass. These explanations are consistent with the formula for a single degree of freedom viscoelastic system (Rao, 1990).

\section{System Loss Factor Versus Core Shear Modulus}

The effects of core shear modulus with $\mathbf{L}_{1} / L=0.2$ are shown in Figure 2.19. As can be seen, the system loss factor varies with the shear modulus $\left(G_{c 1}\right.$ or $\left.G_{c 2}\right)$. If the shear modulus is small, the increase of $\mathrm{G}_{\mathrm{cl}}$ (or $\mathrm{Gc}_{2}$ ) will increase the system loss factor. After some point, if $\mathrm{G}_{\mathrm{cl}}$ (or $\mathrm{G}_{\mathrm{c} 2}$ ) continues to increase, the system loss factor will decrease. Thus, an optimal core shear modulus exists and the trend seen in this figure compares well with the results by $\operatorname{Rao}(1977,1978)$. The optimal value of the core shear modulus is dependent on the system's geometrical and physical parameters. The form of the graph may be explained as follows. For small values of core shear modulus, the increase of core shear modulus will increase the core layer resistance and the energy dissipated, thus increasing the system loss factor. For very large core shear modulus, the damping factor is like the primary beam or the constraining layer, the relative deformation is very small, thus very little energy dissipation occurs. In this case, the loss factor is small. Between the two cases, there exists a value of $G_{c 1}$ or $G_{c 2}$, which results in relatively large shear deformations and a large damping force leading to large energy dissipation. Hence, an optimal 
shear modulus exists. The addition of the end mass acts to counteract the effect of increasing $\mathrm{G}_{c}$. As more mass is added, the loss factor decreases (consistent with the results in Fig. 2.14). Hence, the increase in mass has the overall tendency of decreasing the system loss factor.

Normally, the material properties of damping layers (elastic and shear modulus, loss factor) are functions of temperature and will effect the resonant frequency of the structure. But if the temperature is not changed very much or very fast (for example, a robot arm working at room temperature surroundings), we may consider the material properties to be constant to temperature. However, the results obtained here may be used to determine the correct value, if temperature variations are not large. For example, for a new temperature, a new $\mathrm{G}_{c}$ may be obtained using the manufacturer's specifications for which the system loss factor may be obtained from Fig. 2.19. This loss factor may be used in Fig. 2.18 to obtain the "apparent damping coverage" for a given $\mathrm{M}_{0}$. The application of Fig. 2.18 defines an equivalent system with new coverage length having the same loss factor as the initial system with the new $G_{c}$ but with the same $M_{0}$, all other parameters unchanged. This "apparent" damping coverage may be used in Fig. 2.14 to find the new resonant frequency for the new mass ratio of the equivalent system. 


\subsubsection{Conclusion}

The effects of end mass to system frequency and loss were discussed here. From numerical results, we conclude:

1) Increase of end mass will decrease the resonant frequency;

2) Increase of the coverage length will increase the resonant frequency of the beam at the constant end mass;

3) Increase of the damping layer coverage length will increase the system loss factor of the beam;

4) There exists an optimal damping material shear modulus where the system loss factor takes a maximum value. 


\subsection{Vibration Characteristics of Partially Covered \\ Double Sandwich Cantilever Timoshenko Beam}

\subsubsection{Introduction}

In sections 2.1 and 2.2 , the partially covered double sandwich cantilever beams with or without end mass were studied. However, many effects like shear deformation in constraining layers and primary beam, rotational and longitudinal inertias of all layers, were not included in these models. Because the uncovered Timoshenko beam displays vibration characteristics that are different from those of an uncovered Euler beam, it is necessary to determine difference between the partially covered Timoshenko beam model and partially covered Euler Beam model.

In this section, the differential equations of motion together with the boundary conditions for a partially covered, double sandwich cantilever Timoshenko beam have been derived. The effects of bending and extension, rotational and longitudinal inertia of damping layers; shear deformation, rotational and longitudinal inertia of the constraining layers and the primary beam, are included in the equations. The theory given here is applicable for long as well as short, soft or stiff damping layer double sandwich beams. Also the effects of different parameters on the system loss factor and resonance frequency are discussed. Differences are found to exist with the Euler beam model when 1) the damping layers are stiff; 2) the thickness of the 
damping or constraining layers are large compared to the primary beam thickness;

3) the beam is almost completely or completely covered with damping material; and 4) in the case of higher modes of vibration.

\subsubsection{The Theoretical Model}

The geometry of the double sandwich cantilever beam is shown in Figs 2.20 and 2.21. The following assumption are made in the analysis of the damped part:

1) the beam deflection is uniform across any section;

2) no slip occurs at the interface between layers;

3) plane cross sections in individual layers continue to remain plane after deformation.

Let $u_{i}(i=1,2, \ldots, 5)$ be the longitudinal displacement of $i$ th neutral axis, $\gamma_{i}$ $(i=1,2, \ldots, 5)$ be the shear at the neutral axis and $\psi_{i}(i=1,2, \ldots, 5)$ denote the slope of the deflection curve due to bending. We have the following relationships

$$
\left.\begin{array}{l}
u_{x_{i}}=u_{i}+z_{i} \Psi_{i} \\
u_{z_{i}}=w(x, t)
\end{array}\right\}(i=1,2, \ldots, 5)
$$

Where $u_{x i}$ and $u_{z i}$ represent the longitudinal and transverse displacement in each layer, respectively. The second expression of equation (2-70) is an outcome of the first assumption. From assumption (2), we have 


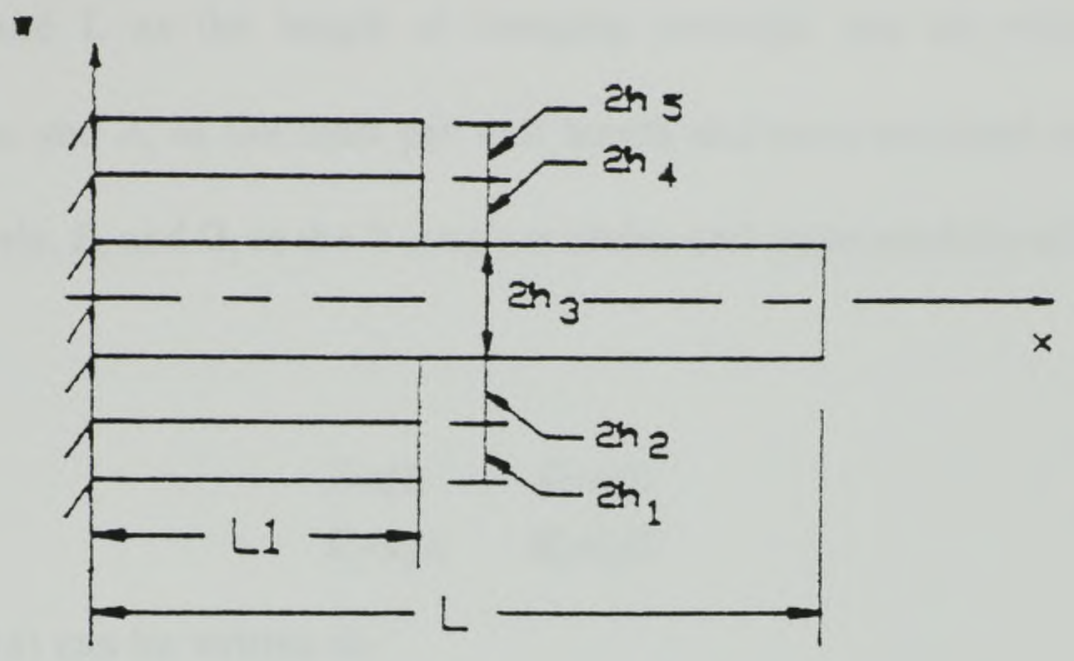

Figure 2.20 Double sandwich beam

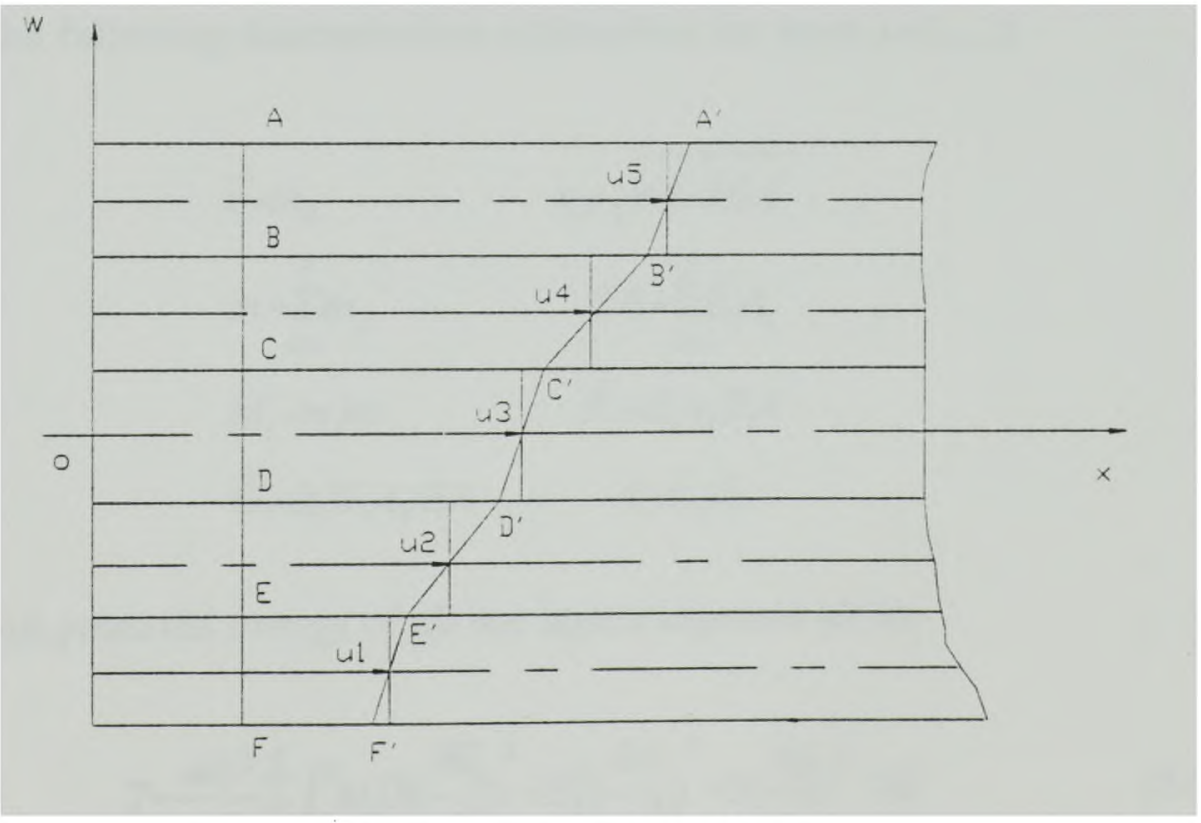

Figure 2.21 Geometry of the beam 


$$
u_{i}+h_{i} \Psi_{i}=u_{i+1}-h_{i+1} \Psi_{i+1} \quad i=1, \ldots, 4
$$

where $\mathrm{h}_{\mathrm{i}}(\mathrm{i}=1,2, \ldots, 5)$ is the half height of $i$ th layer. By defining $\mathrm{k}$ ' as the Timoshenko constant; $L_{1}$ and $L$ as the length of damping coverage and the whole beam respectively; $\mathrm{m}_{\mathrm{i}}$ and $\mathrm{A}_{\mathrm{i}}$ as the mass per unit length and cross sectional area of $i$ th layer respectively; $\mathrm{E}_{\mathrm{i}}$ and $\mathrm{G}_{\mathrm{i}}$ as the Young's modulus and shear modulus of $i$ th layer and by letting

$$
\begin{array}{ll}
\bar{x}=x / L & \bar{w}=w / L \\
\bar{u}_{i}=u_{j} / L & H_{i}=h / L
\end{array}
$$

equation (2-71a) can be written as

$$
\bar{u}_{i}+H_{i} \Psi_{i}=\bar{u}_{i+1}-H_{i+1} \Psi_{i+1} \quad i=1, \ldots, 4
$$

By defining the following dimensionless parameters for layer $i=1, \ldots, 5$

$$
\begin{array}{lc}
\bar{t}=t / t_{0} & t_{0}=\sqrt{m L^{2} / E A} \\
m=\sum_{i=1}^{5} m_{i} & E A=\sum_{i=1}^{5} E_{t} A_{i} \\
M_{i}=m_{i} / m & \bar{E}_{i}=E_{t} A / E A \\
\bar{G}_{i}=k_{i}^{\prime} G_{f} A / E A & \ell=L_{1} / L
\end{array}
$$

the kinetic and potential energy of all the layers together yields

$$
T=\frac{m L^{3}}{6 t_{0}^{2}} \sum_{i=1}^{5} \int_{0}^{1} M_{i}\left(3\left(\frac{\partial \bar{u}_{i}}{\partial t}\right)^{2}+H_{i}^{2}\left(\frac{\partial \Psi_{i}}{\partial t}\right)^{2}+3\left(\frac{\partial \bar{w}}{\partial t}\right)^{2}\right\} d \bar{x}
$$




$$
P=\frac{E A L}{6} \sum_{i=1}^{5} \int_{0}^{e}\left\{\bar{E}_{i}\left[3\left(\frac{\partial \bar{u}_{i}}{\partial \bar{x}}\right)^{2}+H_{i}^{2}\left(\frac{\partial \psi_{i}}{\partial \bar{x}}\right)^{2}+3 \bar{G}_{i}\left(\frac{\partial \bar{w}}{\partial \bar{x}}-\psi_{i}\right)^{2}\right\} d \bar{x}\right.
$$

By Hamilton's principle, we have

$$
\delta \int_{t_{1}}^{t_{2}}(T-P) d t=0
$$

Substituting equations (2-72) and (2-73) into (2-74) and employing the continuity relationships (2-71c) to express $\psi_{1}, \psi_{2}, \psi_{4}, \psi_{5}$ in terms of $\overline{\mathbf{u}}_{\mathrm{i}}$ and $\psi_{3}$ yields the following form of the differential equations of motion for section I (covered part):

$$
[M]\{\ddot{Y}\}+[K]\{Y\}=0
$$

where

$$
\{Y\}=\left\{\bar{u}_{1}, \bar{u}_{2}, \bar{u}_{3}, \bar{u}_{4}, \bar{u}_{5}, \Psi_{3}, \bar{w}\right\}^{T}
$$

$$
[M]=\left\{\begin{array}{ccccccc}
6 M_{1} & -6 M_{1} & 3 M_{1} & 0 & 0 & -3 M_{1} H_{3} & 0 \\
& 12 M_{1}+6 M_{2} & -\left(6 M_{1}+3 M_{3}\right) & 0 & 0 & \left(6 M_{1}+3 M_{2}\right) H_{3} & 0 \\
& & 3 & -\left(3 M_{4}+6 M_{5}\right) & 3 M_{5} & -3 H_{2}\left(M_{1}+M_{2}-M_{4}-M_{5}\right) & 0 \\
& & & 6 M_{4}+12 M_{5} & -6 M_{5} & -\left(3 M_{4}+6 M_{5}\right) H_{3} & 0 \\
& & & & 6 M_{5} & 3 M_{5} H_{3} & 0 \\
& & & & 3 H_{3}^{2} & 0 \\
& & & & & & 3
\end{array}\right\}
$$

and the elements of the $\mathrm{K}$ matrix are 


$$
\begin{aligned}
& k_{11}=4 \bar{E}_{1} \frac{\partial^{2}}{\partial \bar{x}^{2}}-\frac{3 \bar{G}_{1}}{H_{1}^{2}} \\
& k_{13}=\bar{E}_{1} \frac{\partial^{2}}{\partial \bar{x}^{2}}-\frac{3 \bar{G}_{1}}{H_{1}^{2}} \\
& k_{12}=-2 \bar{E}_{1} \frac{\partial^{2}}{\partial \bar{x}^{2}}+\frac{6 \bar{G}_{1}}{H_{1}^{2}} \\
& k_{14}=0 \\
& k_{15}=0 \\
& k_{16}=-\bar{E}_{1} H_{3} \frac{\partial^{2}}{\partial \bar{x}^{2}}+\frac{-3 \bar{G}_{1} H_{3}}{H_{1}^{2}} \\
& k_{17}=\frac{-3 \bar{G}_{1} H_{3}}{H_{1}} \frac{\partial}{\partial \bar{x}} \\
& k_{22}=4\left(\bar{E}_{1}+\bar{E}_{2}\right) \frac{\partial^{2}}{\partial \bar{x}^{2}}-\left(\frac{12 \bar{G}_{1}}{H_{1}^{2}}+\frac{3 \bar{G}_{2}}{H_{2}^{2}}\right) \\
& k_{23}=-\left(2 \bar{E}_{1}+\bar{E}_{2}\right) \frac{\partial^{2}}{\partial \bar{x}^{2}}+\left(\frac{6 \bar{G}_{1}}{H_{1}^{2}}+\frac{3 \bar{G}_{2}}{H_{2}^{2}}\right) \\
& k_{24}=0 \\
& k_{25}=0 \\
& k_{26}=\left(2 \bar{E}_{1}+\bar{E}_{2}\right) H_{3} \frac{\partial^{2}}{\partial \bar{x}^{2}}-\left(\frac{6 \bar{G}_{1}}{H_{1}^{2}}+\frac{3 \bar{G}_{2}}{H_{2}^{2}}\right) H_{3} \\
& k_{27}=\left(\frac{6 \bar{G}_{1}}{H_{1}}-\frac{3 \bar{G}_{2}}{H_{2}}\right) \frac{\partial}{\partial \bar{x}} \quad k_{33}=\left(1+2 \bar{E}_{3}\right) \frac{\partial^{2}}{\partial \bar{x}^{2}}-3\left(\frac{\bar{G}_{1}}{H_{1}^{2}}+\frac{\bar{G}_{2}}{H_{2}^{2}}+\frac{\bar{G}_{4}}{H_{4}^{2}}+\frac{\bar{G}_{5}}{H_{5}^{2}}\right) \\
& k_{34}=-\left(\bar{E}_{4}+2 \bar{E}_{5}\right) \frac{\partial^{2}}{\partial \bar{x}^{2}}+\left(\frac{3 \bar{G}_{3}}{H_{4}^{2}}+\frac{6 \bar{G}_{5}}{H_{5}^{2}}\right) \quad k_{35}=\bar{E}_{5} \frac{\partial^{2}}{\partial \bar{x}^{2}}-\frac{3 \bar{G}_{5}}{H_{5}^{3}} \\
& k_{36}=-\left(\bar{E}_{1}+\bar{E}_{2}-\bar{E}_{4}-\bar{E}_{5}\right) \frac{\partial^{2}}{\partial \bar{x}^{2}}+3\left(\frac{\bar{G}_{1}}{H_{1}^{2}}+\frac{\bar{G}_{2}}{H_{2}^{2}}-\frac{\bar{G}_{4}}{H_{4}^{2}}-\frac{\bar{G}_{5}}{H_{5}^{2}}\right) H_{3} \quad k_{37}=-3\left(\frac{\bar{G}_{1}}{H_{1}}-\frac{\bar{G}_{2}}{H_{2}}+\frac{\bar{G}_{4}}{H_{4}}-\frac{\bar{G}_{5}}{h_{5}}\right) \frac{\partial}{\partial \bar{x}} \\
& k_{44}=4\left(\bar{E}_{4}+\bar{E}_{5}\right) \frac{\partial^{2}}{\partial \bar{x}^{2}}-\left(\frac{3 \bar{G}_{4}}{H_{4}^{2}}+\frac{12 \bar{G}_{5}}{H_{5}^{2}}\right) \quad k_{45}=-2 \bar{E}_{5} \frac{\partial^{2}}{\partial \bar{x}^{2}}+\frac{6 \bar{G}_{5}}{H_{5}^{2}} \\
& k_{46}=-\left(\bar{E}_{4}+2 \bar{E}_{5}\right) H_{3} \frac{\partial^{2}}{\partial \bar{x}^{2}}+\left(\frac{3 \bar{G}_{4}}{H_{4}^{2}}+\frac{6 \bar{G}_{5}}{H_{5}^{2}}\right) H_{3} \quad k_{47}=\left(\frac{3 \bar{G}_{4}}{H_{4}}-\frac{3 \bar{G}}{H_{5}}\right) \frac{\partial}{\partial \bar{x}} \\
& k_{55}=4 \bar{E}_{5} \frac{\partial^{2}}{\partial \bar{x}^{2}}-\frac{3 \bar{G}_{5}}{H_{5}^{2}} \quad k_{56}=\left(\bar{E}_{5} \frac{\partial^{2}}{\partial \bar{x}^{2}}-\frac{3 \bar{G}_{5}}{H_{5}^{2}}\right) H_{3} \\
& k_{57}=\frac{3 \bar{G}_{5}}{H_{5}} \frac{\partial}{\partial \bar{x}} \quad k_{66}=H_{3}^{2} \frac{\partial^{2}}{\partial \bar{x}^{2}}-3\left(\frac{\bar{G}_{1}}{H_{1}^{2}}+\frac{\bar{G}_{2}}{H_{2}^{2}}+\frac{\bar{G}_{3}}{H_{3}^{2}}+\frac{\bar{G}_{4}}{H_{4}^{2}}+\frac{\bar{G}_{5}}{H_{5}^{2}}\right) H_{3}^{2} \\
& k_{67}=3\left(\frac{\bar{G}_{1}}{H_{1}}-\frac{\bar{G}_{2}}{H_{2}}+\frac{\bar{G}_{3}}{H_{3}}-\frac{\bar{G}_{4}}{H_{4}}+\frac{\bar{G}_{5}}{H_{5}}\right) H_{3} \frac{\partial}{\partial \bar{x}} \quad k_{77}=3 \bar{G} \frac{\partial^{2}}{\partial \bar{x}^{2}}
\end{aligned}
$$

and the entries satisfy:

$k_{i j}=k_{j i}, i=1, \ldots, 6 ; j=1, \ldots, 6$. and $k_{i 7}=-k_{7 j}, i=1, \ldots, 6$. 
The dimensionless boundary conditions at $\overline{\mathrm{x}}=0$ are:

$$
\{Y\}=\left\{\bar{u}_{1}, \bar{u}_{2}, \ldots, \bar{u}_{5}, \Psi_{3}, \bar{w}\right\}^{T}=\{0\}^{T}
$$

The dimensionless boundary conditions for the sandwich part(section I) at $\bar{x}=\ell$ are:

$$
\begin{aligned}
& N_{1}^{(1)}=4 \bar{E}_{1} \frac{\partial \bar{u}_{1}}{\partial \bar{x}}-2 \bar{E}_{1} \frac{\partial \bar{u}_{2}}{\partial \bar{x}}+\bar{E}_{1} \frac{\partial \bar{u}_{3}}{\partial \bar{x}}-\bar{E}_{1} H_{3} \frac{\partial \Psi_{3}}{\partial \bar{x}}=0 \\
& N_{2}^{(1)}=-2 \bar{E}_{1} \frac{\partial \bar{u}_{1}}{\partial \bar{x}}+4\left(\bar{E}_{1}+\bar{E}_{2}\right) \frac{\partial \bar{u}_{2}}{\partial \bar{x}}-\left(2 \bar{E}_{1}+\bar{E}_{2}\right) \frac{\partial \bar{u}_{3}}{\partial \bar{x}}+\left(2 \bar{E}_{1}+\bar{E}_{2}\right) H_{3} \frac{\partial \Psi_{3}}{\partial \bar{x}}=0 \\
& N_{3}^{(1)}=\bar{E}_{1} \frac{\partial \bar{u}_{1}}{\partial \bar{x}}-\left(2 \bar{E}_{1}+\bar{E}_{2}\right) \frac{\partial \bar{u}_{2}}{\partial \bar{x}}+\left(1+2 \bar{E}_{3}\right) \frac{\partial \bar{u}_{3}}{\partial \bar{x}}-\left(\bar{E}_{4}+2 \bar{E}_{5}\right) \frac{\partial \bar{u}_{4}}{\partial \bar{x}} \\
& +\bar{E}_{5} \frac{\partial \bar{u}_{5}}{\partial \bar{x}}-\left(\bar{E}_{1}+\bar{E}_{2}-\bar{E}_{4}-\bar{E}_{5}\right) H_{3} \frac{\partial \psi_{3}}{\partial \bar{x}} \\
& N_{4}^{(1)}=-\left(\bar{E}_{4}+2 \bar{E}_{5}\right) \frac{\partial \bar{u}_{3}}{\partial \bar{x}}+4\left(\bar{E}_{4}+\bar{E}_{5}\right) \frac{\partial \bar{u}_{4}}{\partial \bar{x}}-2 \bar{E}_{5} \frac{\partial \bar{u}_{5}}{\partial \bar{x}}-\left(\bar{E}_{4}+2 \bar{E}_{5}\right) H_{3} \frac{\partial \Psi_{3}}{\partial \bar{x}}=0 \\
& N_{5}^{(1)}=\bar{E}_{5} \frac{\partial \bar{u}_{3}}{\partial \bar{x}}-2 \bar{E}_{5} \frac{\partial \bar{u}_{4}}{\partial \bar{x}}+4 \bar{E}_{5} \frac{\partial \bar{u}_{5}}{\partial \bar{x}}+\bar{E}_{5} H_{3} \frac{\partial \Psi_{3}}{\partial \bar{x}}=0 \\
& M^{(1)}=-\bar{E}_{1} H_{3} \frac{\partial \bar{u}_{1}}{\partial \bar{x}}+\left(2 \bar{E}_{1}+\bar{E}_{2}\right) H_{3} \frac{\partial \bar{u}_{2}}{\partial \bar{x}}-\left(\bar{E}_{1}+\bar{E}_{2}-\bar{E}_{4}-\bar{E}_{5}\right) H_{3} \frac{\partial \bar{u}_{3}}{\partial \bar{x}} \\
& -\left(\bar{E}_{4}+2 \bar{E}_{5}\right) H_{5} \frac{\partial \bar{u}_{4}}{\partial \bar{x}}+\bar{E}_{5} H_{3} \frac{\partial \bar{u}_{5}}{\partial \bar{x}}+\bar{E}_{5} H_{3} \frac{\partial \Psi_{3}}{\partial \bar{x}}
\end{aligned}
$$




$$
\begin{aligned}
S^{(1)}=3 \bar{G} \frac{\partial \bar{w}}{\partial \bar{x}}+\frac{3 \bar{G}_{1}}{H_{1}} \bar{u}_{1}+\left(\frac{3 \bar{G}_{2}}{H_{2}}-\frac{6 \bar{G}_{1}}{H_{1}}\right) \bar{u}_{2}+\left(\frac{3 \bar{G}_{1}}{H_{1}}-\frac{3 \bar{G}_{2}}{H_{2}}+\frac{3 \bar{G}_{4}}{H_{4}}-\frac{3 \bar{G}_{5}}{H_{5}}\right) \bar{u}_{3} \\
+\left(\frac{3 \bar{G}_{5}}{H_{3}}-\frac{3 \bar{G}_{4}}{H_{4}}\right) \bar{u}_{4}-\frac{3 \bar{G}_{5}}{H_{5}} \bar{u}_{5}-3\left(\frac{\bar{G}_{1}}{H_{1}}-\frac{\bar{G}_{2}}{H_{2}}+\frac{\bar{G}_{3}}{H_{3}}-\frac{\bar{G}_{4}}{H_{4}}+\frac{\bar{G}_{5}}{H_{5}}\right) H_{3} \Psi_{3}
\end{aligned}
$$

where $\bar{G}=\bar{G}_{1}+\bar{G}_{2}+\bar{G}_{3}+\bar{G}_{4}+\bar{G}_{5}$. Here the $N_{i}^{(1)}, M^{(1)}$ and $S^{(1)}$ represent the generalized axial force in each layer, the generalized moment for the cross-section and the generalized shear for the cross-section, respectively, in the sandwich part of the beam. The differential equations of motion for the uncovered portion of the beam( Section II) are:

$$
\begin{gathered}
-M_{3} \frac{\partial^{2} \bar{u}_{3}^{\prime}}{\partial \vec{t}^{2}}+\bar{E}_{3} \frac{\partial^{2} \bar{u}_{3}^{\prime}}{\partial \bar{x}^{2}}=0 \\
-M_{3} H_{3}^{2} \frac{\partial^{2} \Psi_{3}^{\prime}}{\partial \bar{t}^{2}}+\bar{E}_{3} H_{3}^{2} \frac{\partial^{2} \Psi_{3}^{\prime}}{\partial \bar{x}^{2}}+3 \bar{G}_{3}\left(\frac{\partial \bar{w}^{\prime}}{\partial \bar{x}}-\Psi_{3}^{\prime}\right)=0 \\
-M_{3} \frac{\partial^{2} \bar{w}^{\prime}}{\partial \bar{t}^{2}}+\bar{G}_{3}\left(\frac{\partial^{2} \bar{w}^{\prime}}{\partial \bar{x}^{2}}-\frac{\partial \Psi_{3}^{\prime}}{\partial \bar{x}}\right)=0
\end{gathered}
$$

All primed quantities, ( )', represent the variables in the uncovered portion of the beam. The boundary conditions for the uncovered portion of the beam at $\bar{x}=\ell$ are

$$
N_{3}^{(2)}=3 \bar{E}_{3} \frac{\partial \bar{u}_{3}^{\prime}}{\partial \bar{x}}
$$




$$
\begin{gathered}
M^{(2)}=\bar{E}_{3} H_{3}^{2} \frac{\partial \Psi_{3}^{\prime}}{\partial \bar{x}} \\
S^{(2)}=-3 \bar{G}_{3}\left(\frac{\partial \bar{w}^{\prime}}{\partial \bar{x}}-\Psi_{3}^{\prime}\right)
\end{gathered}
$$

The interface between Section I (covered part of the beam) and section II (uncovered part of the beam) requires that $S^{(1)}+S^{(2)}=0, M^{(1)}+M^{(2)}=0$ and $\mathrm{N}_{3}{ }^{(1)}+\mathrm{N}_{3}{ }^{(2)}=0$ at $\overline{\mathrm{x}}=\ell$. Also the displacement and slope at the interface must be continuous:

$$
\begin{gathered}
\bar{w}=\bar{w}^{\prime} \\
\bar{w}=\bar{w}^{\prime}, \quad \frac{\partial \bar{w}}{\partial \bar{x}}-\psi_{3}=\frac{\partial \bar{w}^{\prime}}{\partial \bar{x}}-\psi_{3}^{\prime}
\end{gathered}
$$

The dimensionless boundary conditions at $\bar{x}=1$ are:

$$
\left\{\frac{\partial \bar{u}_{3}^{\prime}}{\partial \bar{x}}, \frac{\partial \Psi_{3}^{\prime}}{\partial \bar{x}}, \frac{\partial \bar{w}^{\prime}}{\partial \bar{x}}-\Psi_{3}^{\prime}\right\}=\{0,0,0\}
$$

indicating that no extensional force, moment and shear force exist at the end of the beam.

\subsubsection{Frequency and Loss Factor}

To solve the governing differential equation of motion of the system, we assume

$$
Y=Y_{n} e^{\lambda \cdot \bar{x}} e^{j \alpha_{n}^{*} \bar{t}}
$$


where $Y$ and $Y_{n}$ are defined as

$$
\begin{gathered}
Y=\left[\bar{u}_{1}, \bar{u}_{2}, \ldots, \bar{u}_{5}, \Psi_{3}, \bar{w}\right]^{T} \\
Y_{n}=\left[\bar{u}_{1 n}, \bar{u}_{2 n}, \ldots, \bar{u}_{5 n}, \Psi_{3 n}, \bar{w}_{n}\right]^{T}
\end{gathered}
$$

and $\lambda^{*}$ depends upon the value of $\Omega_{\mathrm{a}}{ }^{*}$, as discussed below. Substituting $\mathrm{Y}$ into the governing equation (2-75), nontrivial solutions exist if the corresponding determinant is zero. Thus

$$
\operatorname{det}\left(-[M] \Omega_{n}^{* 2}+[K]\right)=0
$$

where the $\partial / \partial \bar{x}$ and $\partial^{2} / \partial \vec{x}^{2}$ terms in $[\mathrm{K}]$ are replaced by $\lambda^{*}$ and $\lambda^{* 2}$, respectively. Similarly, for section II, we have the following characteristic equation generated from equation (2-78):

$$
\begin{gathered}
M_{3} \Omega_{n}^{* 2}+\bar{E}_{3} \lambda^{* 2}=0 \\
\operatorname{det}\left\{\begin{array}{cc}
M_{3} H_{3}^{2} \Omega_{n}^{* 2}+\bar{E}_{3} H_{3}^{2} \lambda^{* 2}+3 \bar{G}_{3} & 3 \bar{G}_{3} \lambda^{*} \\
-3 \bar{G}_{3} \lambda^{*} & 3 \Omega_{n}^{* 2}+3 \bar{G}_{3} \lambda^{* 2}
\end{array}\right\}=0
\end{gathered}
$$

where $\lambda^{*}$ are characteristic values. For any $\Omega_{\mathrm{n}}^{*}$, let $\lambda_{\mathrm{n}, 1}, \lambda_{\mathrm{n}, 2}, \ldots, \lambda_{\mathrm{n}, 14^{*}}$ denote the fourteen solutions of equation (2-82); let $\lambda_{\mathrm{n}, 15}{ }^{*}, \lambda_{\mathrm{n}, 16}, \lambda_{\mathrm{n}, 17}, \lambda_{\mathrm{n}, 18}{ }^{*}$ denote the four zeros of equation (2-83b); and let $\lambda_{\mathrm{n}, 19^{*}}, \lambda_{\mathrm{n}, 20}{ }^{*}$ denote the two zeros of equation (2-83a). These values may be determined from the boundary conditions and continuity conditions.

For section II, the spatial part of the solution is defined as: 


$$
\left.\begin{array}{l}
\bar{w}_{n}^{\prime}(\bar{x})=\sum_{i=15}^{18} A_{n i} e^{\lambda_{n, n^{*}} \bar{x}} \\
\Psi_{3 n}^{\prime}(\bar{x})=\sum_{i=15}^{18} A_{n i} e^{\lambda_{n i}^{*} \bar{x}} \\
\bar{u}_{3 n}^{\prime}(\bar{x})=\sum_{i=19}^{20} A_{n i} e^{\lambda_{n, n^{*}}^{*} \bar{x}}
\end{array}\right\}
$$

For section I, the spatial part of the solution is defined as:

$$
\left.\begin{array}{l}
\bar{u}_{1 n}(\bar{x})=\sum_{i=1}^{14} A_{n i} U_{1 i} e^{\lambda_{n,}^{*} \bar{x}} \\
u_{2 n}(\bar{x})=\sum_{i=1}^{14} A_{n i} U_{2 i} e^{\lambda_{n,}^{*} \bar{x}} \\
u_{3 n}(\bar{x})=\sum_{i=1}^{14} A_{n i} U_{3 i} e^{\lambda_{n, \bar{x}} \bar{x}} \\
u_{4 n}(\bar{x})=\sum_{i=1}^{14} A_{n i} U_{4 i} e^{\lambda_{n,}^{*} \bar{x}} \\
u_{5 n}(\bar{x})=\sum_{i=1}^{14} A_{n i} U_{5 i} e^{\lambda_{n,}^{*} \bar{x}} \\
\bar{w}_{n}(\bar{x})=\sum_{i=1}^{14} A_{n i} e^{\lambda_{n, i} \bar{x}} \\
\Psi_{3 n}(\bar{x})=\sum_{i=1}^{14} A_{n i} \phi_{i} e^{\lambda_{i n} \bar{x}}
\end{array}\right\}
$$

The $\mathrm{A}_{\mathrm{i}} \mathrm{s}(\mathrm{i}=1,2, \ldots, 20)$ have to be determined from the boundary conditions and the continuity conditions at the interface between the covered part and the uncovered part. We have a total of 20 homogeneous equations involving the unknowns $A_{i}$. For nontrivial solution, the corresponding determinant must be zero, i.e.,

$$
\operatorname{det}\left(\Omega_{n}^{*}, \lambda^{*}\right)=0
$$

Equations (2-82), (2-83) and (2-86) are nonlinear complex equations for unknowns $\Omega_{\mathrm{n}}^{*}$ and $\lambda^{*}$ (the characteristic value). We define the complex resonance frequency 
factor, $\mathrm{p}^{*}$, the real resonance frequency factor, $\mathrm{p}$, and the system loss factor, $\eta$, of the beam as follows:

$$
\left.\begin{array}{l}
p^{*}=\Omega_{n}^{*} t_{0}=p \sqrt{1+j \eta} \\
p=\Omega_{n} t_{0}=\sqrt{\operatorname{Re}\left(p^{* 2}\right)} \\
\eta=\operatorname{Im}\left(p^{* 2}\right) / \operatorname{Re}\left(p^{* 2}\right)
\end{array}\right\}
$$

A modified Muller's method was developed to find $k_{n}^{*}$ and $p^{*}$, thus yielding the resonance frequency factor and loss factor.

\subsubsection{Comparisons of Numerical Results}

Numerical results for different parameters were obtained and are displayed as graphs. The input parameters employed in the previously described numerical scheme, unless stated otherwise, were:

$$
\begin{gathered}
\ell=\mathrm{L}_{1} / \mathrm{L}=0.2, \rho_{1}=\rho_{3}=\rho_{5}=7850.0 \mathrm{Kg} / \mathrm{m}^{3}, \rho_{2}=\rho_{4}=3140.0 \mathrm{Kg} / \mathrm{m}^{3}, \\
\mathrm{H}_{1}=\mathrm{H}_{2}=\mathrm{H}_{3}=\mathrm{H}_{4}=\mathrm{H}_{5}=0.02, \overline{\mathrm{E}}_{1}=\overline{\mathrm{E}}_{3}=\overline{\mathrm{E}}_{5}=0.30, \overline{\mathrm{E}}_{2}=\overline{\mathrm{E}}_{4}=3 \overline{\mathrm{G}}_{2}, \overline{\mathrm{G}}_{3}=\overline{\mathrm{G}}_{5}=\overline{\mathrm{E}}_{1} / 2.5, \\
\overline{\mathrm{G}}_{2}=\overline{\mathrm{G}}_{4}=\mathrm{G}_{\mathrm{r} 2}\left(1+\mathrm{j} \eta_{2}\right), \mathrm{G}_{\mathrm{r} 2}=0.001, \eta_{2}=\eta_{4}=1.2, \mathrm{k}_{\mathrm{i}}=0.833 .
\end{gathered}
$$

The values of $\eta_{2}$ and $\eta_{4}$ are representative of high damping materials and $\mathrm{G}_{\mathrm{r} 2}$ is the real part of $\bar{G}_{2}$. The values of $k_{1}$ through $k_{s}$ are taken as being equal to 0.833 for the sake of simplicity and more will be said about the $\mathrm{k}_{\mathrm{i}} \mathrm{s}$ when the effect of the Timoshenko factor is discussed. Finally, the $E_{i} s$ and $G_{i} s$ are connected via the Poisson ratio. The Poisson ratio of the primary beam is assumed to be 0.25 and the Poisson ratio of the damping layers is assumed to be 0.5 . 


\section{Effect of Damping Layer Height}

Figure 2.22 shows the system loss factor versus the damping layer height ratio (the height of the damping layer to the length of the beam, $\mathrm{H}_{2}$, or $\mathrm{H}_{4}$ ). In general, the system loss factor for the Timoshenko beam model is less than that for the Euler beam model. Also if $\mathrm{H}_{2}$ is larger than about 0.04 , any increase of the damping layer height ratio will increase the difference in system loss factor between the Euler and Timoshenko beam models. Also there exists an optimal value of $\mathrm{H}_{2}$ that produces the highest system loss factor. The location of the optimal results for the parameters chosen compare very well with results reported elsewhere (Levy and Chen, 1994a).

Figure 2.23 shows the resonance frequency factor versus the damping layer height ratio. It may be seen from the figure that, for small ratios of $\mathrm{H}_{2}$ (less than 0.02 ), the frequency factor for the two models is almost the same; for large ratios of $\mathrm{H}_{2}$ (larger than 0.02 ), the Timoshenko beam model gives smaller values compared with the Euler beam model. The difference between the two models increases with increase in the ratio, $\mathrm{H}_{2}$. Hence, for small ratio of $\mathrm{H}_{2}$, the Euler beam model compares well with the Timoshenko beam model. From the figure we note that the resonance frequency factor predicted by the Timoshenko beam model is smaller than that predicted by Euler beam model. This trend wherein the Timoshenko beam model gives smaller resonance frequency factor values than those of the Euler beam model compares well with results reported (Weaver, et al 1990) where no damping treatment was used. The reasons for these trends may be explained as follows: 




Figure 2.22 System loss factor versus damping layer height ratio

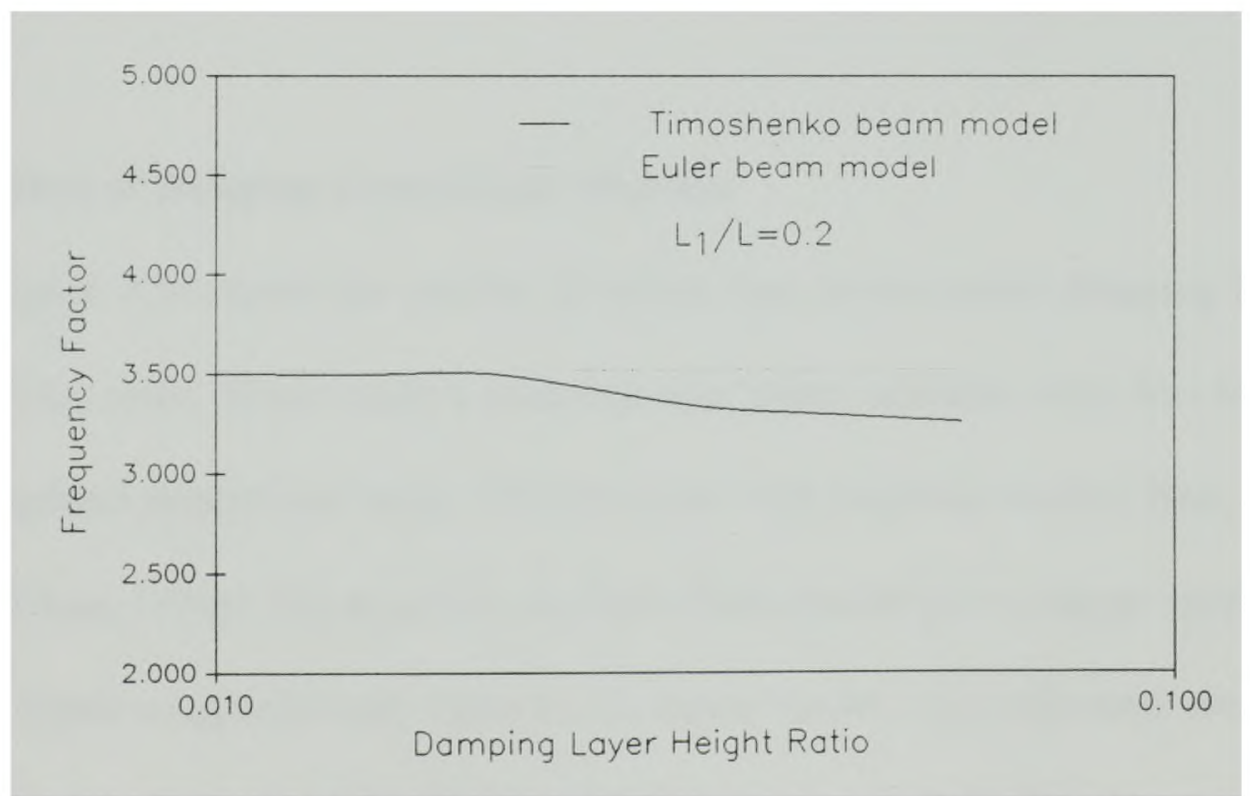

Figure 2.23 Frequency factor versus damping layer height ratio 
Given a covered Euler and covered Timoshenko beam having equal total energy, we suppose that both execute the same end displacement. In the covered Euler beam model, the shear effect was only accounted for in the damping layers. In the present Timoshenko beam model, the shear effect, extension and bending as well as rotatory and extensional inertia are accounted for. Because more energy would be needed to deform the damping layers in the Timoshenko beam model than in the Euler beam model, less energy would be available to vibrate the primary beam. Since the energy for an equivalent single degree of freedom system is directly proportional to the square of the natural frequency and amplitude of vibration, this would require the covered Timoshenko beam model that is executing the same end displacement to vibrate at a lower frequency.

\section{Effect of Damping Layer Shear Modulus}

Figure 2.24 shows the results of system loss factor versus damping layer shear modulus ratio. There exists a damping layer shear modulus ratio that will produce an optimal system loss factor. This coincides with previous results ( Rao, 1978; Levy and Chen, 1994a). We note that the Euler beam model gives a larger value of system loss factor compared with Timoshenko beam model, especially near the maximum system loss factor. In other words, the Euler beam model overestimates the system loss factor.

Figure 2.25 shows the results of resonance frequency factor versus damping layer 


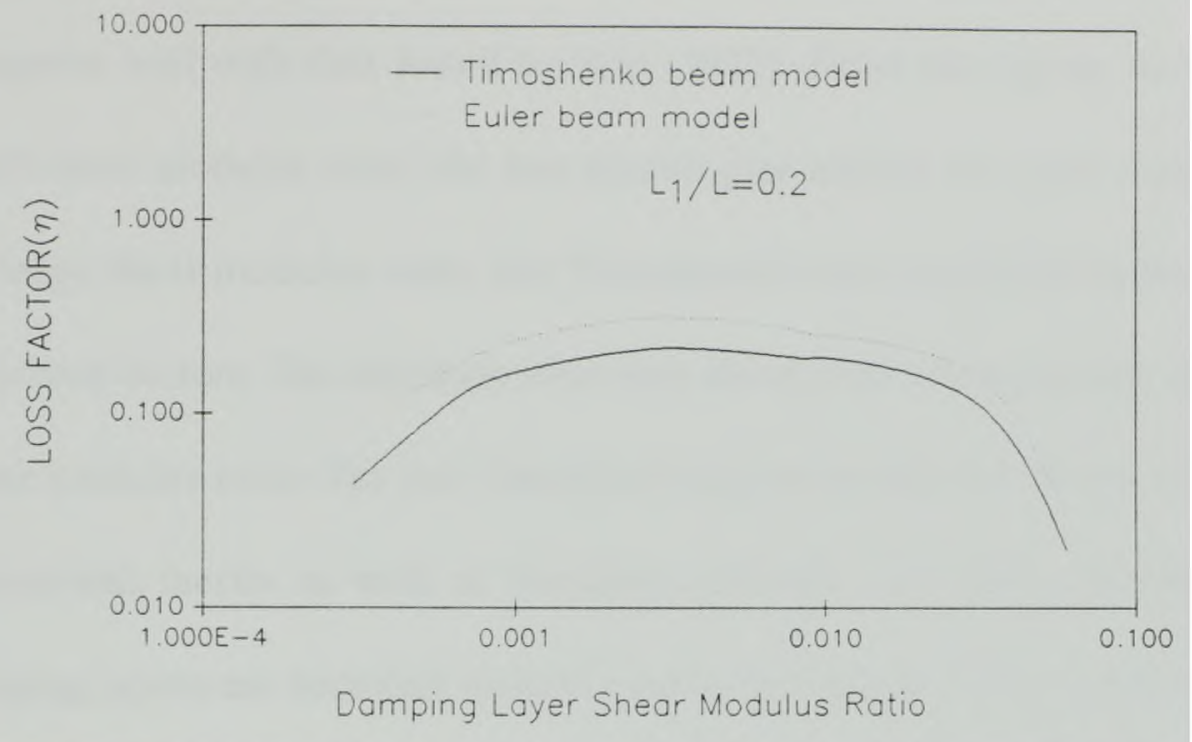

Figure 2.24 System loss factor versus damping layer shear modulus ratio

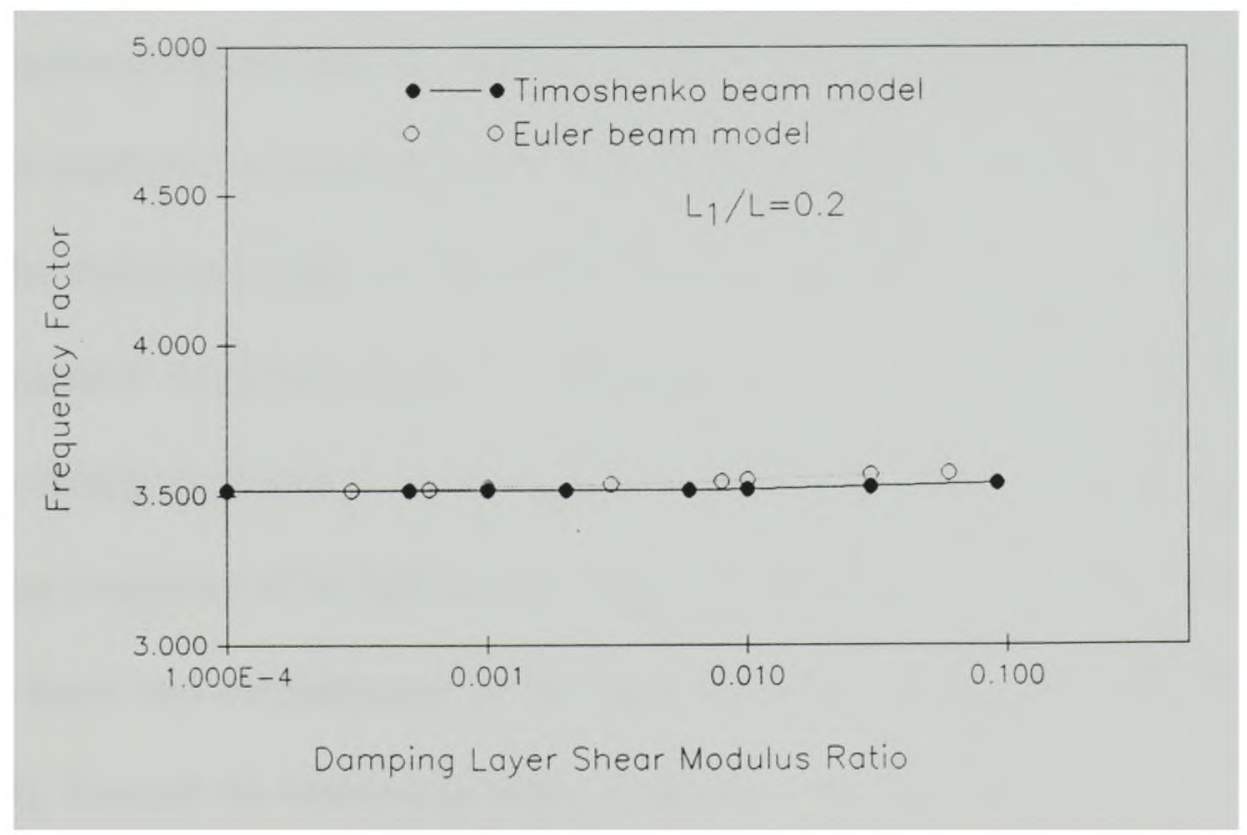

Figure 2.25 Frequency factor versus damping layer shear modulus ratio 
shear modulus ratio. The resonance frequency factor predicted by the Timoshenko beam model is smaller than that predicted by Euler beam model. This trend compares well with that found by Rao (1977). From the figure, we note that for small shear modulus ratio, the two models give almost the same results. However, for large shear modulus ratio, the Timoshenko beam model gives lower resonance frequency factors. The difference between the models increases with the increase in shear modulus ratio. For our Timoshenko beam model, the effects of rotatory and extensional inertia as well as bending, extension and shear deformation in the damping layers are included while for our Euler beam model only the shear effects in the damping layers are accounted for. If the value of damping layer shear modulus ratio is small, that implies that the Young's modulus is small. Thus, the bending and extensional effects may be neglected. However, for large values of damping layer shear modulus, we need to take these effects into account. Increase in shear modulus of the damping layers, and thus their Young's modulus as well, increases the energy required to bend and extend the damping layers of our Timoshenko model leaving less energy to vibrate the primary beam. This leads to a lower resonance frequency factor compared to an Euler beam model having the same total energy and executing the same end displacement as we have seen previously (see discussion of Figure 2.23). Overall the increase in shear modulus of the damping layers will increase the Young's modulus of the damping layers, thus increasing the bending stiffness of the entire system causing an increase in the frequency factor for both models. 


\section{Effect of Constraining Layer Height}

The effects of constraining layer height ratio, $\mathrm{H}_{5}$, on the system loss factor is shown in Figure 2.26. For the Euler beam model, there is an optimal value of $\mathrm{H}_{5}$ (or $\mathrm{H}_{1}$ ); for the Timoshenko beam model, the value of system loss factor is smaller compared with Euler beam model, but the trend in the data is almost the same. As the constraining layer thickness ratio increases, more energy is required to deform the thicker constraining layer. This will increase the system loss factor until a maximum point is reached, then the value decreases. This trend is similar in nature to the trend reported for the covered Euler beam model (Levy and Chen, 1994).

\section{Effect of Sandwich Coverage Length}

Figure 2.27 shows the system loss factor versus coverage length ratio of the sandwich part $\left(\ell=\mathrm{L}_{1} / \mathrm{L}\right)$. For small value of coverage ratio, the two models give almost the same results. As the coverage increases, differences in the system loss factor between the two models occur with Euler beam model predicting higher values of system loss factor. We see that for small coverage, omitting the effect of shear in the constraining layers and the rotatory and longitudinal inertia effects of the whole beam will not be harmful. However, for large coverage, these effects must be included. From the figure we note that an increase in coverage will increase the 




Figure 2.26 System loss factor versus constraining layer thickness ratio

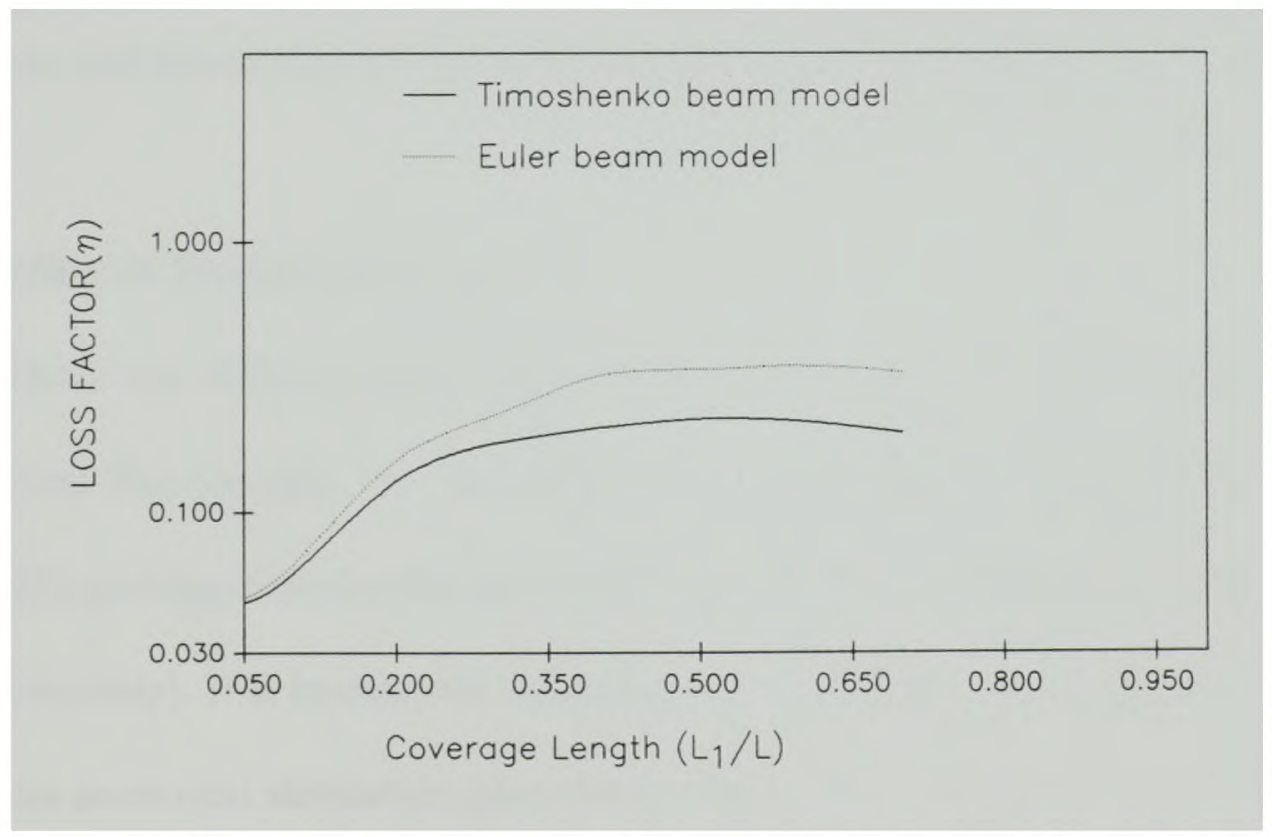

Figure 2.27 System loss factor versus coverage length ratio 
system loss factor to some maximum value. This trend follows the trend of the covered Euler beam model (Levy and Chen, 1994a). However, the maximum for the Timoshenko model occurs at a smaller coverage length than for the covered Euler beam model.

\section{Effect of Vibration Mode Number}

Figure 2.28 shows the results of system loss factor versus the mode number. In general, the Euler beam model predicts higher values of system loss factor compared with Timoshenko beam model. From the figure we note that even the first mode predicts differences between the two models, but the difference is small compared with the higher modes. Coverage length also affects the difference in the system loss factor and the effects are more pronounced in the first few modes.

\section{Effect of Timoshenko Factor, k'}

There are different opinions on the proper value of $\mathrm{k}^{\prime}$ for a rectangular cross section. For example, for the same cross section, Weaver et al. (1990) and Fung (1965) quoting Timoshenko, give different values to be used $\left(k^{\prime}=0.833\right.$ and $k^{\prime}=2 / 3$, respectively). It is known that the actual k' values may be different than those used in the numerical simulation given here. Furthermore, the k' values for the damping and constraining layers may not be the same as those of the primary beam. Yet, even though the k' values may be different from layer to layer, the choice of $k$ ' will have 


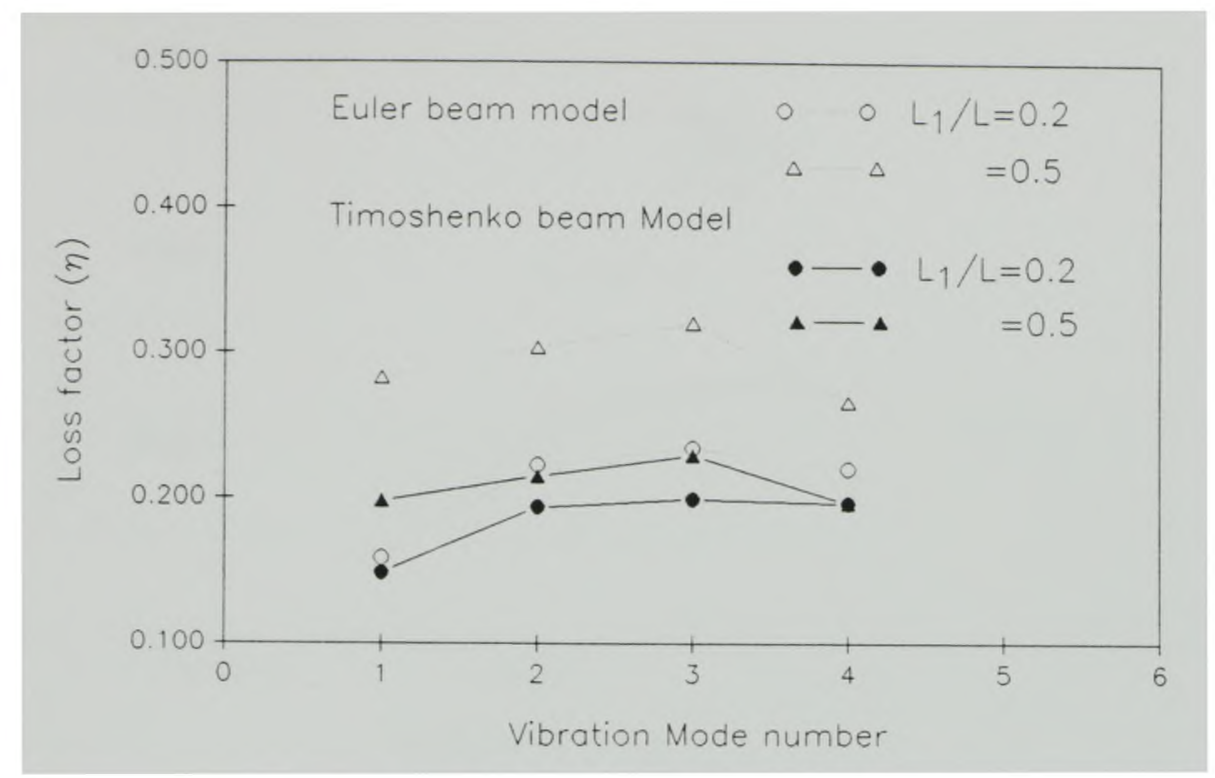

Figure 2.28 System loss factor versus vibration mode



Figure 2.29 The effects of Timoshenko factor to frequency factor 


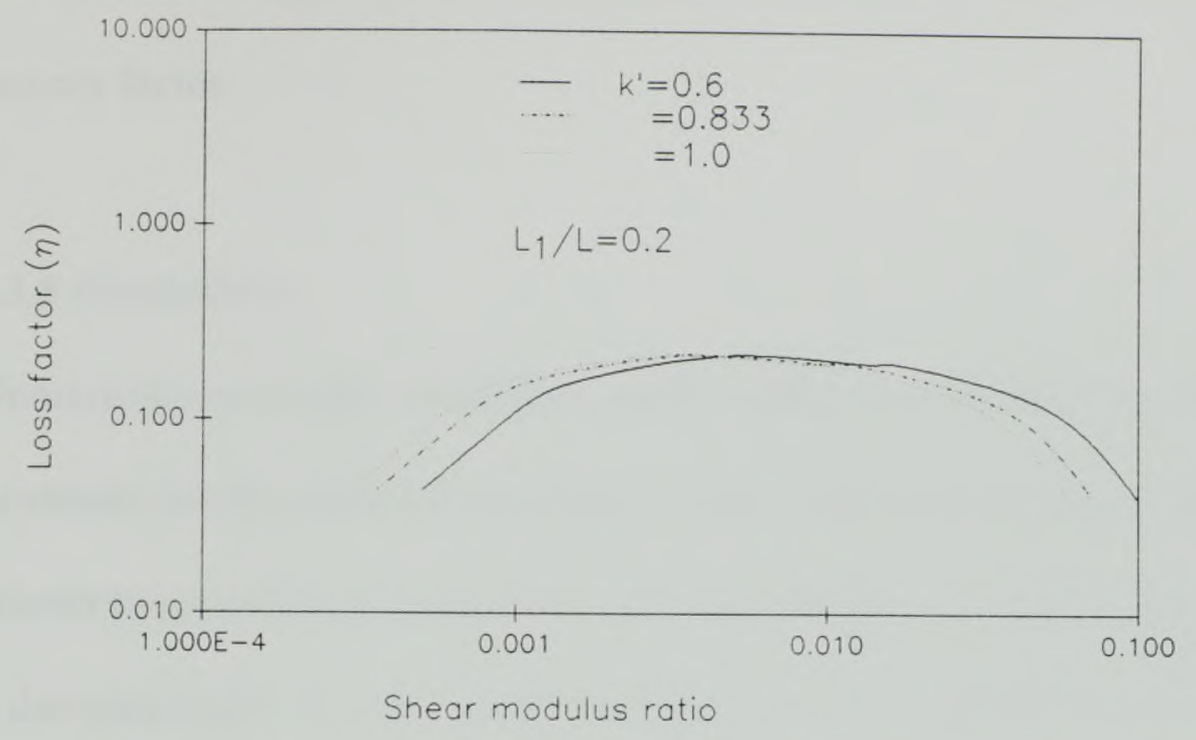

Figure 2.30 The effects of Timoshenko factor to system loss factor

an effect on the resonance frequency factor (Figure 2.29) and the system loss factor (Figure 2.30). In these two figures, the $\mathrm{k}_{\mathrm{i}}$ 's are assumed to be the same. For small value of $\bar{G}_{2}$, the resonance frequency factor is unaffected by choice of $k_{i}$, but for large value of $\bar{G}_{2}$, larger value of $k_{i}$ ' will have larger resonance frequency factor (see Figure 2.29).

The value of $k_{i}$ also has an important effect on the system loss factor. In the region, $0.005<\bar{G}_{2}<0.011$, the system loss factor is virtually unaffected by the value of k' (see Figure 2.30). However, for $\bar{G}_{2}>0.011$, the increase of k' will decrease the value of the system loss factor, and for $\bar{G}_{2}<0.005$, the increase of k' will increase the value of the system loss factor. For example, at $\bar{G}_{2}=0.05$, an increase of $k_{i}^{\prime}$ of $40 \%$ 
will cause the system loss factor to change by about $30 \%$. Thus, the proper choice of k' may have an important effect on the system loss factor as well as the resonance frequency factor.

\subsubsection{Conclusions}

Numerical results show that there will be differences between the results of the Timoshenko double sandwich cantilever beam model and the Euler double sandwich cantilever beam model given by Levy and Chen (1994) in the following situations: 1) stiff damping layers; 2) large partially or fully covered sandwich beam; 3) thickness of damping layers or constraining layers are big compared with the thickness of primary beam; 4) higher modes vibration. In these cases, it may be better to use the results from the Timoshenko beam model. The differences exist as a result of the effects of bending and extension, rotational and longitudinal inertia of the damping layers, and the shear deformation, rotational and longitudinal inertia of the constraining layers and primary beam, which are included in the governing differential equations. The theory given here is applicable for long as well as short, soft or stiff damping layers double sandwich beams. 
Chapter 3 Active vibration control of elastic beam by means of shape memory alloy layers

\subsection{Introduction}

In recent years, improvements in materials and manufacturing process, and more specifically advances in data acquisition and computer technologies, have made it possible to apply sophisticated active control techniques to vibration structures (Browning and Medaugh, 1992). One method of vibration control is by use of shape memory alloys (SMA). Buehler and Wiley (1965) first discovered the shape memory effect of nickel-titanium alloys. It has also been shown that composites with SMA have the ability to change material properties, induce large internal forces in the materials, modify the stress and strain state of the structure and alter the configuration (Rogers, et al., 1989). These characteristics are also useful for vibration control.

Brinson (1993) presented one-dimensional constitutive behavior of shape memory alloys. A three-dimensional constitutive model for shape memory alloys was given by Graesser and Cozzarelli (1994). Others have investigated applications of SMA composites for vibration and structural acoustic control (Rogers, 1990; Saunders, et al., 1990; Rogers, et al., 1991). It was shown that, for a beam with SMA fibers having a nitinol volume of $15 \%$, the first natural frequency of the beam 
increased from $21 \mathrm{~Hz}$ at room temperature to $62 \mathrm{~Hz}$ when the actuators were heated to $300^{\circ} \mathrm{F}\left(149^{\circ} \mathrm{C}\right)$ (Rogers, et al., 1991). The use of SMA have spawned many different areas. For examples, Nagaya, et al (Nagaya, et al, 1987), studied the vibration control of rotating shafts passing through critical speeds by changing the stiffness of the supports with use of memory metals. Liang and Rogers (1993) presented a design method of shape memory springs for vibration control. The application of SMA for aseismic isolation were studied by Graesser and Cozzarelli (1991). Yet, when vibration control of an existing structure is necessary, it may not be feasible to replace parts of the structure or to modify them. However, it may be feasible to overlay the structure with SMA layers in combination with a constrained viscoelastic layer (CVL) to modify the vibration characteristics of the structure (Chen and Levy, 1994b, 1995a, 1995b). Because of the effectiveness of the combined SMA/CVL control technique, it is desired to evaluate the effectiveness of SMA layers by themselves as a vibration control method.

In this chapter, the mathematical model of a flexible beam covered with shape memory alloy layers is presented. Numerical results shows that change of the elastic modulus of the shape memory alloy layer will affect the natural frequency of the beam. A linear control law is determined based on optimal regulator theory. The control results are discussed. Because the use of SMA layer needs heating and cooling cycle, the temperature effects are also discussed. 


\subsection{Formulations of the Problem}

\subsubsection{Governing equation of the beam}

The beam is shown in Figure 3.1. The lower and upper sides are bonded with shape memory alloy layers which are used as actuators. To simplify the analysis, the following assumptions are made in the analysis:

1) the SMA layers are very thin;

2) Euler theory is used in the beam analysis;

3) no slip occurs at the interface between the layers; and

4) the thermal effects due to temperature changes used to control the SMA layers are neglected (These effects will be considered later on).

From force balance and neglecting terms higher than first order, we have the following equations:

$$
\left.\begin{array}{c}
\frac{\partial \sigma_{1}}{\partial x} h_{1}-\tau_{1}=0 \\
\frac{\partial \sigma_{3}}{\partial x} h_{3}+\tau_{2}=0 \\
\frac{\partial}{\partial x}\left(E_{2} b h_{2} \frac{\partial u_{2}}{\partial x}\right)+\tau_{1} b-\tau_{2} b=0 \\
\frac{\partial Q}{\partial x}+m \frac{\partial^{2} w}{\partial t^{2}}=0 \\
\frac{\partial M}{\partial x}-Q-\frac{1}{2} \tau_{1} b h_{2}-\frac{1}{2} \tau_{2} b h_{2}=0
\end{array}\right\}
$$




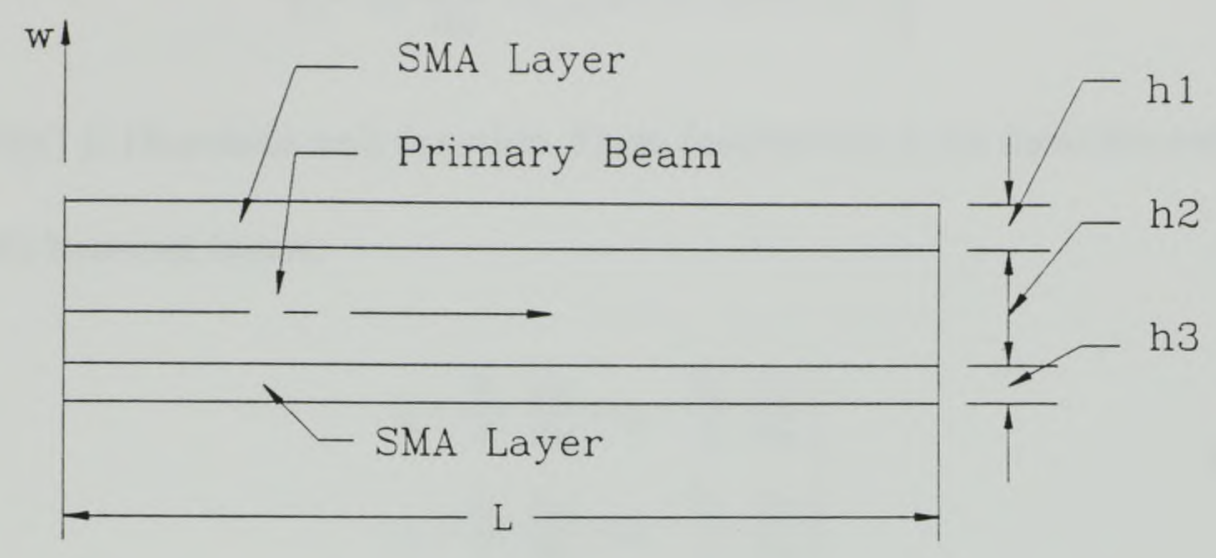

Figure 3.1 Configuration of Beam with SMA Layers

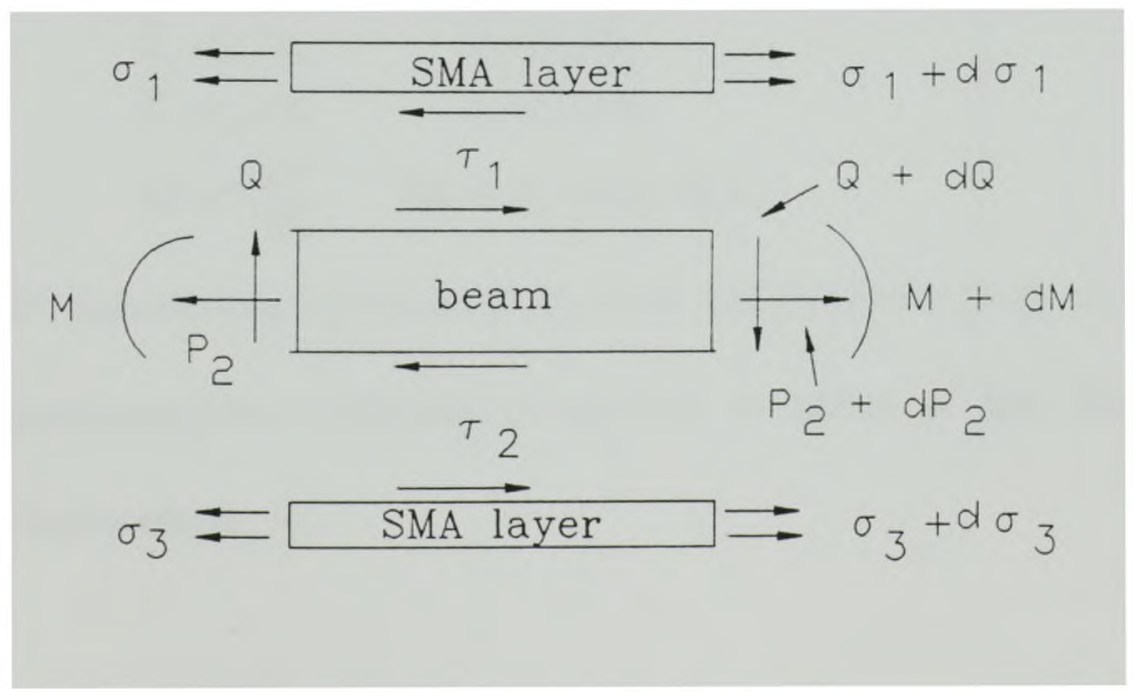

Figure 3.2 Forces and Stresses on Beam Element 
The stresses in the SMA layers are given by (Rogers, et al., 1991)

$$
\left.\begin{array}{l}
\sigma_{1}=E_{1} \frac{\partial u_{1}}{\partial x}+\sigma_{z 1}[H(x)-H(x-L)] \\
\sigma_{3}=E_{3} \frac{\partial u_{3}}{\partial x}+\sigma_{r 3}[H(x)-H(x-L)]
\end{array}\right\}
$$

Where $\mathrm{H}(\mathrm{x})$ is Heaviside unit function. From assumption 3, we have the continuity conditions between layers:

$$
\left.\begin{array}{l}
u_{1}+\frac{h_{1}}{2} \frac{\partial w}{\partial x}=u_{2}-\frac{h_{2}}{2} \frac{\partial w}{\partial x} \\
u_{2}+\frac{h_{2}}{2} \frac{\partial w}{\partial x}=u_{3}-\frac{h_{3}}{2} \frac{\partial w}{\partial x}
\end{array}\right\}
$$

The parameters defined in equations (3-1)-(3-3) are defined in the list of symbols. Introducing the following nondimensional parameters

$$
\begin{array}{rcr}
\bar{x}=\mathrm{x} / \mathrm{L} & \overline{\mathrm{t}}=\mathrm{t} / \mathrm{t}_{0} & \overline{\mathrm{w}}=\mathrm{w} / \mathrm{L} \\
\mathrm{H}_{1}=\mathrm{h}_{1} / \mathrm{h}_{2} & \mathrm{H}_{2}=\mathrm{h}_{2} / \mathrm{L} & \mathrm{H}_{3}=\mathrm{h}_{3} / \mathrm{h}_{2} \\
\overline{\mathrm{E}}_{1}=\mathrm{E}_{1} / \mathrm{E}_{2} & \bar{E}_{3}=\mathrm{E}_{3} / \mathrm{E}_{2} & \\
\bar{\sigma}_{1}=\sigma_{\mathrm{r} 1} / \mathrm{E}_{2} & \bar{\sigma}_{3}=\sigma_{\mathrm{r} 3} / \mathrm{E}_{2} & \\
\mathrm{t}_{0}^{2}=\mathrm{K}^{2} \frac{\mathrm{mL}}{\mathrm{EA}} & \mathrm{EA}=\mathrm{E}_{1} \mathrm{~A}_{1}+\mathrm{E}_{2} \mathrm{~A}_{2}+\mathrm{E}_{3} \mathrm{~A}_{3}
\end{array}
$$

where $\kappa=10^{5}$. Substituting equations (3-2), (3-3) and (3-4) into (3-1), we obtain the following nondimensional differential equation in terms of the dimensionless transverse displacement, $\bar{w}$, 


$$
\frac{\mathrm{B}_{5} \mathrm{H}_{2}^{2}}{\mathrm{~B}_{1}} \frac{\partial^{4} \overline{\mathrm{W}}}{\partial \overline{\mathrm{x}}^{4}}+\frac{\partial^{2} \overline{\mathrm{W}}}{\partial \overline{\mathrm{t}}^{2}}=\frac{\mathrm{B}_{6}}{\mathrm{~B}_{1}}\left[\bar{\sigma}_{1}-\frac{\mathrm{H}_{1}}{\mathrm{H}_{3}} \bar{\sigma}_{3}\right]\left[\delta^{\prime}(\overline{\mathrm{x}})-\delta^{\prime}(\overline{\mathrm{x}}-1)\right]
$$

where $\delta(x)$ is the Dirac delta function and the prime means differentiation with respect to the coordinate $\bar{x}$.

$$
\begin{aligned}
& \mathrm{B}_{1}=1+\overline{\mathrm{E}}_{1} \mathrm{H}_{1}+\overline{\mathrm{E}}_{3} \mathrm{H}_{3} \\
& \mathrm{~B}_{2}=\frac{1}{2}\left[\left(1+\mathrm{H}_{1}\right) \overline{\mathrm{E}}_{1} \mathrm{H}_{1}-\left(1+\mathrm{H}_{3}\right) \overline{\mathrm{E}}_{3} \mathrm{H}_{3}\right] \\
& \mathrm{B}_{3}=\frac{1}{12}+\frac{1}{4}\left[\left(1+\mathrm{H}_{1}\right) \overline{\mathrm{E}}_{1} \mathrm{H}_{1}+\left(1+\mathrm{H}_{3}\right) \overline{\mathrm{E}}_{3} \mathrm{H}_{3}\right] \\
& \mathrm{B}_{4}=\frac{1}{2}\left[\overline{\mathrm{E}}_{1} \mathrm{H}_{1}-\overline{\mathrm{E}}_{3} \mathrm{H}_{3}\right] \\
& \mathrm{B}_{5}=\frac{1}{12}+\mathrm{B}_{3}-\frac{\mathrm{B}_{2} \mathrm{~B}_{4}}{\mathrm{~B}_{1}} \\
& \mathrm{~B}_{6}=\frac{1}{2} \mathrm{H}_{1} \mathrm{H}_{2}\left(1-\frac{2 \mathrm{~B}_{4}}{\mathrm{~B}_{1}}\right)
\end{aligned}
$$

In the case of a simply supported beam, the boundary conditions are:

$$
\bar{w}(\bar{x}, \bar{t})=0, \quad \frac{\partial^{2} \bar{w}(\bar{x}, \bar{t})}{\partial \bar{x}^{2}}=0 \quad \text { at } \bar{x}=0,1
$$

It can be noted that changing the elastic modulus of the SMA layer will change the coefficients of the equations, thus changing the natural frequency of the beam. Also if we change the recovery stress of each SMA layer, $\sigma_{r i}$, we can modify the excitation to the flexible beam. These changes may be accomplished by varying the temperature of the SMA layers. 
3.2.2 Modal analysis in state space variables

To control the motion of the beam, it is necessary to determine its displacement. This is accomplished by positing that the problem may be approached from a separation of variables point of view. Thus, the transverse displacement response of the beam is assumed to be of the form:

$$
\bar{w}(\bar{x}, \bar{t})=\sum_{i=1}^{\infty} T_{i}(\bar{t}) \phi_{i}(\bar{x}),
$$

where $T_{i}(\bar{t})$ is the time dependent function to be determined and $\Phi_{i}(\bar{x})$ is the eigenfunctions of the beam and are taken as $\sin \lambda_{i} x$ to satisfy the boundary conditions given by (3-6). Substituting equation (3-7) into equation (3-5) and using the orthogonal relationships for eigenfunctions yield:

$$
\ddot{T}_{1}(\bar{t})+A_{i} T_{1}(\bar{t})=D_{1} u(\bar{t})
$$

where

$$
\begin{aligned}
& \mathrm{A}_{i}=\frac{\mathrm{B}_{5} \mathrm{H}_{2}^{2} \lambda_{i}^{4}}{\mathrm{~B}_{1}} \quad \mathrm{D}_{i}=\frac{\mathrm{B}_{6} \lambda_{i}}{\mathrm{~B}_{1}}\left[1-(-1)^{i}\right] \\
& \mathrm{u}(\overline{\mathrm{t}})=\bar{\sigma}_{1}(\bar{t})-\frac{\mathrm{H}_{1}}{\mathrm{H}_{3}} \bar{\sigma}_{3}(\bar{t})
\end{aligned}
$$

The solution $\bar{w}$ is composed of an infinite sums of terms. Yet the effect of the higher modes may be very small. We may, therefore, assume that only the first $n$ modes have significant influence on $\bar{w}$. Thus we may assume a $2 n$-dimensional state variable vector to be: 


$$
\mathrm{x}_{c}(\bar{t})=\left[\mathrm{T}_{1}(\bar{t}), \ldots, \mathrm{T}_{n}(\bar{t}) ; \dot{\mathrm{T}}_{1}(\bar{t}), \ldots, \dot{\mathrm{T}}_{n}(\bar{t})\right]^{T}
$$

where the superscript, $T$, denotes the transpose of the vector. With the above state variables, equation (3-8) may be written as:

$$
\dot{X}_{c}(\bar{t})=A_{c} X_{c}(\bar{t})+D_{c} u(\bar{t}),
$$

where

$$
A_{c}=\left[\begin{array}{cc}
0 & I \\
-\delta_{i j} A_{i} & 0
\end{array}\right], \quad D_{c}=\left[\begin{array}{cc}
0 & 0 \\
0 & \delta_{i j} D_{i}
\end{array}\right] .
$$

$I$ is the identity matrix and $\delta_{i j}$ is the Kronecker delta. If a sensor is attached at $\bar{x}=$ $\bar{x}_{\xi}$, the corresponding deflection at that point is $\bar{w}_{\xi}\left(\bar{x}_{\xi}, \bar{t}\right)$. The output is then given by:

$$
Y_{c}=d_{c} X_{c}(\bar{t})
$$

where $d_{c}$ is a constant vector and $d_{c}=\left[\Phi_{1}\left(\bar{x}_{\xi}\right), \ldots, \Phi_{n}\left(\bar{x}_{\xi}\right) ; 0, \ldots, 0\right]$.

\subsection{Temperature Effects}

\subsubsection{Thermal bending moment}

To include the temperature effect, we will relax assumption (4). As shown in the Figure 3.3, we assume the temperatures at the upper interface and lower interface of the beam and SMA layers to be $T_{1}$ and $T_{3}$, respectively. Neglecting the longitudinal heat conduction, the temperature distribution inside the beam 


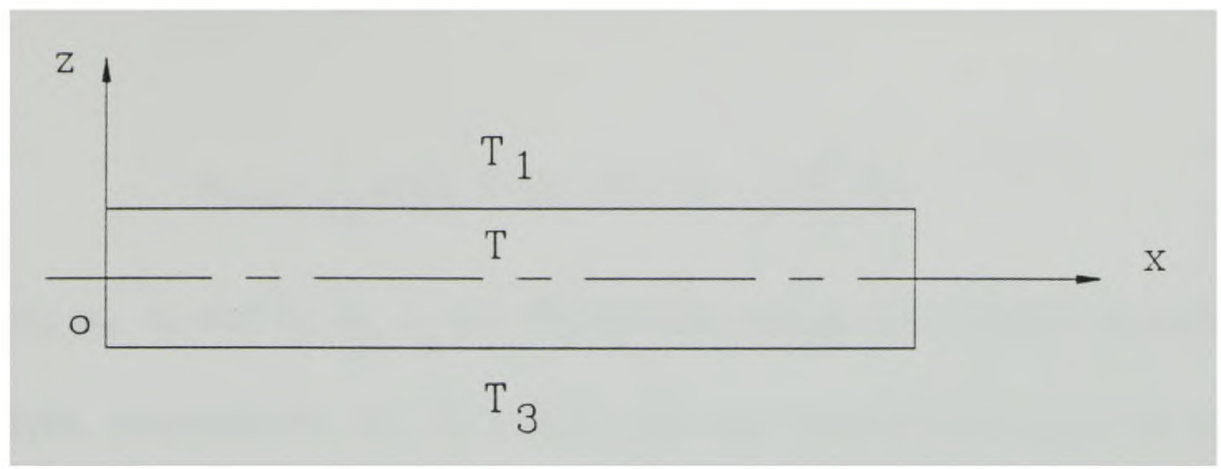

Figure 3.3 Temperature Distribution

section is determined by solving the one-dimensional unsteady heat conduction problem

$$
\frac{\partial T(z, t)}{\partial t}=\alpha \frac{\partial^{2} T(z, t)}{\partial z^{2}}
$$

under the boundary and initial conditions

$$
\begin{cases}T(z, t)=T_{1} & \text { at } z=h / 2 \\ T(z, t)=T_{3} & \text { at } z=-h / 2 \\ T(x, t)=T_{0} & \text { when } t=0\end{cases}
$$

Where $\alpha$ is the thermal diffusivity, and $\mathrm{T}_{0}$ is the initial temperature. The temperature distribution within the beam along $z$-coordinate is found to be:

$$
\begin{aligned}
& T(z, t)=\frac{1}{2}\left(T_{1}+T_{3}\right)+\left(T_{1}-T_{3}\right) \frac{z}{h_{2}}+ \\
& \frac{2}{\pi} \sum_{i=1}^{\infty} \frac{e^{-\alpha \beta_{i}^{2} t}}{i} \sin \beta_{i}\left(z+\frac{h_{2}}{2}\right)\left[\left(T_{0}-T_{3}\right)+\left(T_{1}-T_{0}\right)(-1)^{i}\right]
\end{aligned}
$$

The thermal bending moments on the beam, $\mathrm{M}_{\mathrm{SMA}}$ and $\mathrm{M}_{\text {beam }}$, are found to be 


$$
\begin{gathered}
M_{S M A} \doteq \frac{1}{2} h_{2} b\left[E_{3} \alpha_{3} h_{3}\left(T_{3}-T_{0}\right)-E_{1} \alpha_{1} h_{1}\left(T_{1}-T_{0}\right)\right] \\
M_{\text {beam }}=\frac{1}{12} b h_{2}^{2} \alpha_{2} E_{2}\left(T_{1}-T_{3}\right)\left(1-\frac{6}{\pi^{2}} \sum_{i=1}^{\infty} \frac{1}{i^{2}} e^{-\alpha\left(\frac{2 i \pi}{h_{2}}\right)^{2} t}\right)
\end{gathered}
$$

where $\alpha_{1}, \alpha_{2}, \alpha_{3}$ and $\mathrm{h}_{1}, \mathrm{~h}_{2}, \mathrm{~h}_{3}$ are the thermal expansion coefficient and height of each layer, respectively; $E_{1}, E_{2}$ and $E_{3}$ are the Young's modulus of each layer, respectively; and $\mathrm{b}$ is the width of the beam. Since the temperature is a function of time, $M_{S M A}$ and $M_{\text {beam }}$ are time functions. Let $M_{T}(t)=M_{S M A}+M_{\text {beam }}$ where $M_{T}(t)$ is constant along $\mathrm{x}$ direction. The bending moment may be expressed in terms of the Heaviside unit function $\mathrm{H}(\mathrm{x})$

$$
M_{T}(x, t)=[H(x)-H(x-L)] M_{T}(t)
$$

\subsubsection{Governing Equation of the Beam}

The stresses in the SMA layers when the temperature is included are:

$$
\left.\begin{array}{l}
\sigma_{1}=E_{1}\left[\varepsilon_{1}+\alpha_{1}\left(T_{1}-T_{0}\right)\right]+\sigma_{r 1}=E_{1}\left[\frac{\partial u_{1}}{\partial x}+\alpha_{1}\left(T_{1}-T_{0}\right)\right]+\sigma_{r 1} \\
\sigma_{3}=E_{3}\left[\varepsilon_{3}+\alpha_{3}\left(T_{3}-T_{0}\right)\right]+\sigma_{r 3}=E_{3}\left[\frac{\partial u_{3}}{\partial x}+\alpha_{3}\left(T_{3}-T_{0}\right)\right]+\sigma_{r 3}
\end{array}\right\}
$$

The Young's modulus, $\mathrm{E}_{1}, \mathrm{E}_{3}$ and the recovery stress, $\sigma_{\mathrm{r} 1}, \sigma_{\mathrm{r} 3}$ of the SMA layers are functions of temperature, strain and martensitic fraction $\xi$. The Young's modulus of 
the SMA layers can be expressed as follows (Lagoudas, et al., 1994):

$$
\left\{\begin{array}{l}
E_{1}=\xi_{1} E_{M 1}+\left(1-\xi_{1}\right) E_{A 1} \\
E_{3}=\xi_{3} E_{M 3}+\left(1-\xi_{3}\right) E_{A 3}
\end{array}\right.
$$

where $E_{M i}$ and $E_{A i}(i=1,3)$ are the martensite and austenite Young's modulus of the $i$-th layer, respectively. The martensite fraction, $\xi_{i}(i=1,3)$, can be expressed as (Nagaya and Hirata, 1992):

$$
\begin{cases}\xi_{i}=1-\exp \left[\mathrm{a}_{M i}\left(\mathrm{M}_{s i}-\mathrm{T}_{i}\right)+\mathrm{b}_{M i} \sigma_{i}\right] & \mathrm{M}_{f i} \leq \mathrm{T} \leq \mathrm{M}_{s i} \\ \xi_{i}=\exp \left[\mathrm{a}_{A i}\left(\mathrm{~A}_{s i}-\mathrm{T}_{i}\right)+\mathrm{b}_{A i} \sigma_{i}\right] & \mathrm{A}_{s i} \leq \mathrm{T} \leq \mathrm{A}_{f i}\end{cases}
$$

Where $M_{s i}$ and $M_{f i}(i=1,3)$ are the temperatures at the beginning of the martensite transformation and at the end of the martensite transformation, respectively. $A_{s i}$ and $A_{\mathfrak{f i}}(i=1,3)$ are the corresponding temperatures for the austenite transformation. $a_{\text {Mi}}$, $b_{M i}, a_{A i}$ and $b_{A i}(i=1,3)$ are material constants. From equations (3-1), (3-3), (3-16) and (3-17), we obtain the following equation in terms of the transverse displacement, $w(x, t):$

$$
B_{5} \frac{\partial^{4} W}{\partial x^{4}}+m \frac{\partial^{2} W}{\partial t^{2}}=\left[B_{6} \sigma_{r 1}+B_{7} \sigma_{r 3}+M_{T}\right]\left[\delta^{\prime}(x)-\delta^{\prime}(x-L)\right]
$$

where $\delta(x)$ is the Dirac delta function and the prime means differentiation with respect to the coordinate $x$. If we introduce the nondimensional parameters defined in Equation (3-4), then Equation (3-20) can be written as follows: 


$$
\frac{\bar{B}_{5} H_{2}^{2}}{12 \mathrm{D}} \frac{\partial^{4} \overline{\mathrm{W}}}{\partial \overline{\mathrm{X}}^{4}}+\frac{\partial^{2} \overline{\mathrm{W}}}{\partial \overline{\mathrm{t}}^{2}}=\frac{\bar{B}_{6} \mathrm{H}_{2}}{2 \mathrm{D}}\left[\mathrm{H}_{1} \bar{\sigma}_{1}-\mathrm{H}_{3} \bar{\sigma}_{3}+2 \overline{\mathrm{M}}_{t}\right]\left[\delta^{\prime}(\overline{\mathrm{X}})-\delta^{\prime}(\overline{\mathrm{X}}-1)\right]
$$

The coefficients defined in the equation are:

$$
\begin{aligned}
& \overline{\mathrm{D}}=\overline{\mathrm{B}}_{1}=1+\overline{\mathrm{E}}_{1} \mathrm{H}_{1}+\overline{\mathrm{E}}_{3} \mathrm{H}_{3} \\
& \overline{\mathrm{B}}_{2}=\overline{\mathrm{E}}_{1} \mathrm{H}_{1}\left(1+\mathrm{H}_{1}\right)-\overline{\mathrm{E}}_{3} \mathrm{H}_{3}\left(1+\mathrm{H}_{3}\right) \\
& \overline{\mathrm{B}}_{3}=1+3\left[\overline{\mathrm{E}}_{1} \mathrm{H}_{1}\left(1+\mathrm{H}_{1}\right)+\overline{\mathrm{E}}_{3}\left(1+\mathrm{H}_{3}\right) \mathrm{H}_{3}\right] \\
& \overline{\mathrm{B}}_{4}=\overline{\mathrm{E}}_{1} \mathrm{H}_{1}-\overline{\mathrm{E}}_{3} \mathrm{H}_{3} \\
& \mathrm{~B}_{5}=1+\overline{\mathrm{B}}_{3}-3 \overline{\mathrm{B}}_{2} \overline{\mathrm{B}}_{4} / \overline{\mathrm{B}}_{1} \\
& \overline{\mathrm{B}}_{6}=1-\overline{\mathrm{B}}_{4} / \overline{\mathrm{B}}_{1} \\
& \overline{\mathrm{M}}_{\mathrm{t}}=\mathrm{M}_{T} / \mathrm{bh}_{2}^{2} \mathrm{E}_{2}
\end{aligned}
$$

and ( )' is differentiation with respect to $\bar{x}$. In the case of a simply supported beam, the boundary conditions are defined by Equation (3-6). It can be noted that changing the elastic modulus of the SMA layers will change the coefficients of the equations, thus changing the natural frequency of the beam. Also if we change the recovery stress of each SMA layer, $\sigma_{\mathrm{ri}}$, and temperature gradient, we can modify the excitation to the flexible beam. These changes may be accomplished by varying the temperature of the SMA layers. For the symmetrical case, $h_{1}=h_{3}, T_{1}=T_{3}, E_{1}=E_{3}, \alpha_{1}=\alpha_{3}$, the right side of equation (11) would be zero. In this case, there is no control on the excitation, but we can still "tune" the natural frequency of the system by changing the Young's modulus. 


\subsubsection{Modal analysis in state space variables}

If we assume a variable seperable solution, the transverse displacement of the beam is given by Equation (3-7). The eigenfunctions of the beam are taken as $\sin \lambda_{i} x$ to satisfy the boundary conditions given by (3-6). Substituting equation (3-7) into equation (3-21) and using the orthogonal relationships for eigenfunctions yield:

$$
\ddot{T}_{i}(\bar{t})+A_{i} T_{i}(\bar{t})=D_{i} u(\bar{t})
$$

where

$$
\left.\begin{array}{l}
A_{1}=\frac{\bar{B}_{5} H_{2}^{2} \lambda_{1}^{4}}{12 D_{1}} \quad D_{1}=\lambda_{1} H_{2}\left[1-(-1)^{i}\right] \\
u(\bar{t})=\frac{\bar{B}_{6}}{2 D}\left[H_{1} \bar{\sigma}_{1}(\bar{t})-H_{3} \bar{\sigma}_{3}(\bar{t})+2 \bar{M}_{T}\right]
\end{array}\right\}
$$

Substituting the above $A_{i}$ and $D_{i}$ into equation (3-10a), we obtain new $A_{c}$ and $D_{c}$.

\subsection{Optimal control}

We assume that the design objective is to return the system response from its initial state to the system equilibrium position. In order to determine the feedback state vector, digital optimal regulatory theory will be applied. The performance index for the linear regulator problem is defined to be: 


$$
J=\frac{1}{2} \int_{\bar{t}_{0}}^{\infty}\left(X_{c}^{T} Q X_{c}+u^{T} R u\right) d \bar{t}
$$

where $Q$ and $R$ are symmetric positive definite weighting matrices. An approximate choice of these matrices must be made to obtain "acceptable" levels of $x\left(\bar{t}_{f}\right), x(\bar{t})$, and $u(\bar{t})$, where $\bar{t}_{f}$ means terminal time. Assume that the desired optimal control law is

$$
u^{*}(\bar{t})=-R^{-1} D_{c}^{T} S(\bar{t}) X^{*}(\bar{t})
$$

where $X^{*}(\bar{t})$ is the solution of the state equation with optimal control $u^{*}$ as input, and $S(\bar{t})$ is a symmetric matrix to be determined by the following Riccati matrix equation:

$$
Q-\mathrm{SD}_{c} \mathrm{R}^{-1} \mathrm{D}_{c}^{T} \mathrm{~S}+\mathrm{A}_{c}^{T} \mathrm{~S}+\mathrm{SA} \mathrm{A}_{c}=\dot{\mathrm{S}}
$$

Since we are interested in controlling the steady state vibration or controlling the structure over a longtime interval, $\dot{S}$ in the Riccati equation is set to be zero.

\subsection{Numerical Results}

The values chosen for the numerical computation are $(\mathrm{Pu}, 1995)$ :

$$
\begin{gathered}
\mathrm{h}_{2} / \mathrm{L}=0.01, \mathrm{~h}_{1} / \mathrm{h}_{2}=0.1, \mathrm{~h}_{3} / \mathrm{h}_{2}=0.1, \mathrm{E}_{\mathrm{A} 1} / \mathrm{E}_{2}=\mathrm{E}_{\mathrm{A} 3} / \mathrm{E}_{2}=0.368, \\
\mathrm{E}_{\mathrm{M} 1} / \mathrm{E}_{2}=\mathrm{E}_{\mathrm{M} 3} / \mathrm{E}_{2}=0.117, \mathrm{M}_{\mathrm{f} 1}=\mathrm{M}_{\mathrm{f} 3}=5.0^{\circ} \mathrm{C}, \mathrm{M}_{\mathrm{s} 1}=\mathrm{M}_{\mathrm{s} 3}=23.0^{\circ} \mathrm{C}, \\
\mathrm{A}_{\mathrm{s} 1}=\mathrm{A}_{\mathrm{s} 3}=29.0^{\circ} \mathrm{C}, \mathrm{A}_{\mathrm{f} 1}=\mathrm{A}_{\mathrm{f} 3}=51.0^{\circ} \mathrm{C}, \mathrm{T}_{0}=25^{\circ} \mathrm{C}
\end{gathered}
$$




\subsubsection{Frequency Factor}

The results displayed in Figures $3.4-3.7$ show the effects of different parameters on the natural frequency of the system. In the figures, the vertical axis represents the nondimensional natural frequency (frequency factor) of the system.

Figure 3.4 shows the temperature effects of SMA on the frequency factor of the system. If the temperature of the SMA layer is less than the austenite start temperature, the increase of the temperature will decrease the system frequency factor. Beginning at the austenite start temperature, the temperature increase of SMA layer will increase the frequency factor. For the temperature above the austenite finish temperature, the temperature increase of SMA will decrease the frequency factor. This is because beginning at the austenite start temperature, the phase transformation due to temperature increase will increase the Young's modulus of the SMA layers, thus causing an increase of frequency factor. After the austenite finish temperature, no further increase of Young's modulus will occur. On the other hand, the increase of temperature will have the effect of decreasing the system frequency factor. Hence, a decrease in frequency factor is observed after the austenite finish temperature.

The effects of SMA layer thickness ratio (the ratio of SMA layer thickness to beam thickness) to system frequency factor may be seen in Figures 3.4 or 3.5 . Generally speaking, increase of the ratio will increase the system frequency factor, 


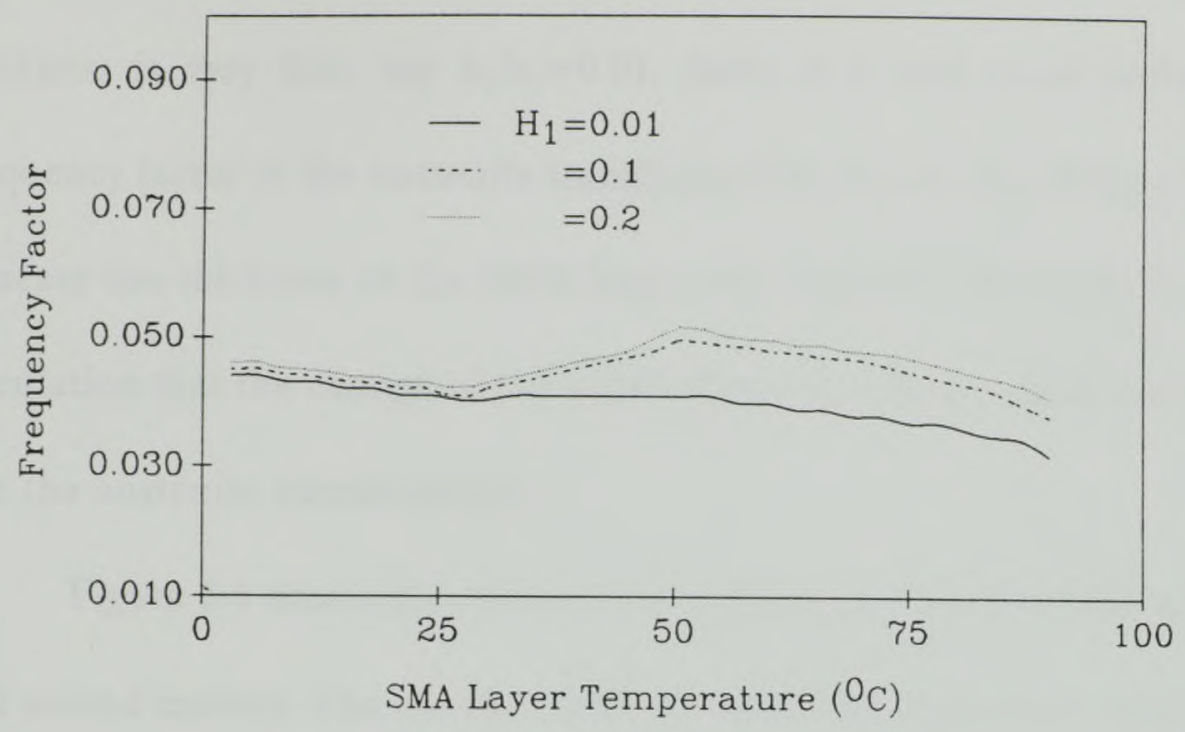

Figure 3.4 SMA layer temperature vs. system frequency factor

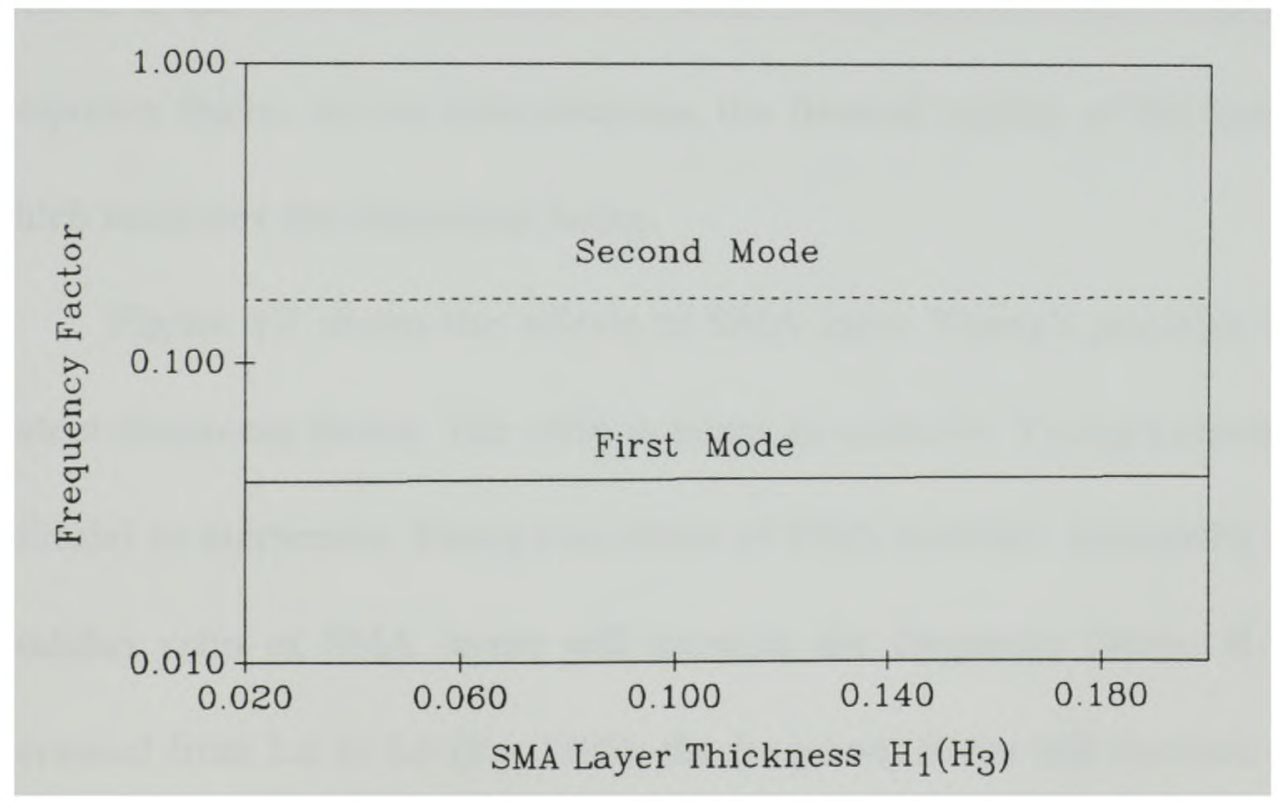

Figure 3.5 SMA layer thickness vs. system frequency factor 
both for first mode frequency and for second mode frequency (see Figure 3.5). To observe the thickness effect, we assume a constant temperature $\mathrm{T}=25^{\circ} \mathrm{c}$ ). If the thickness is very thin, say $h_{1} / h_{2}=0.01$, there is a very small increase in system frequency factor at the austenite transformation process (see Figure 3.4). But if we increase the thickness of the SMA layer, say $h_{1} / h_{2}=0.2$, we found in the numerical calculation that the change of the system frequency factor could be as high as $20 \%$ due the austenite transformation.

Figure 3.6 shows the effects of the ratio $h_{2} / L$ on the frequency factors (first and second modes). The increase of the ratio will increase the frequency factor. For very small ratio, the frequency factor is small. The reason may be explained as follows. If the ratio is very small, the beam is very flexible, thus resulting in a small frequency factor. As the ratio increase, the flexural rigidity of the beam increases, which increases the frequency factor.

Figure 3.7 shows the effects of SMA layer Young's modulus ratio on the system frequency factor. The ratio is taken as austenite Young's modulus of SMA material to martensite Young's modulus of SMA material. Increasing the Young's modulus ratio of SMA layers will increase the frequency factor. If the ratio is increased from 1.0 to $6.0(\mathrm{Pu}, 1995)$, the frequency factor will increase about $5 \%$. 


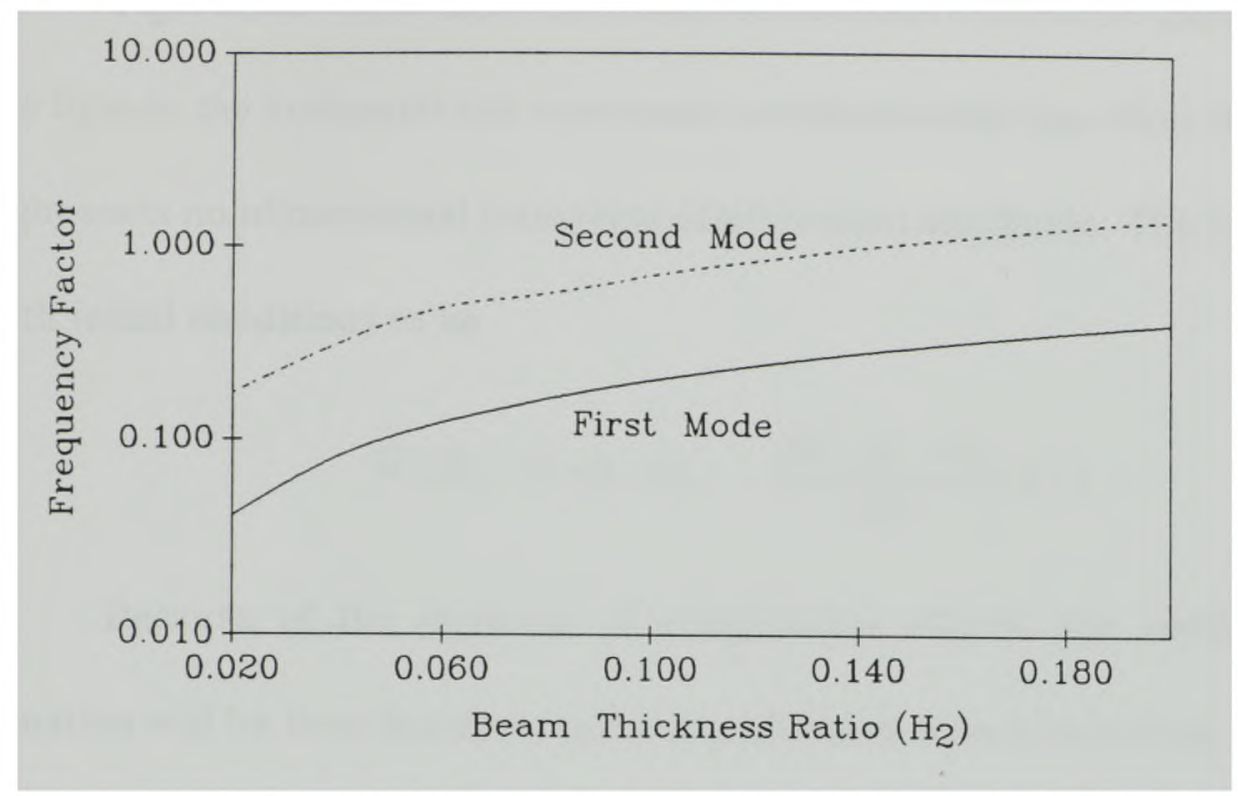

Figure 3.6 Beam thickness ratio $h_{2} / L$ vs. frequency factor



Figure 3.7 SMA Young's modulus ratio vs. system frequency factor 


\subsubsection{Control results}

Figures $3.8-3.10$ show the results of vibration control for different cases. In the figures, the horizontal axis represents nondimensional time while the vertical axis represents nondimensional transverse displacement amplitude. The beam oscillates with initial conditions to be

$$
\bar{w}(\bar{x}, 0)=0.05, \quad \frac{\partial \bar{w}(\bar{x}, 0)}{\partial t}=0.0
$$

Because of the inclusion of temperature effects, the coefficients in the equation will be time functions and the problem is solved iteratively. Given a time increment and the initial conditions, we try to obtain displacement and velocity. Then using the solution as new initial conditions, we move to the next time step. We continue the process until completion.

Figure 3.8 compares the results with and without control. In the figure, the solid and dotted lines represent the results with and without control, respectively. It can be seen from the figure that in the case of no control, the beam will oscillate continuously while for the case of control, the vibration will die out very quickly $(\mathfrak{\tau}=0.15)$. The response time has been reduced tremendously compared with the case 


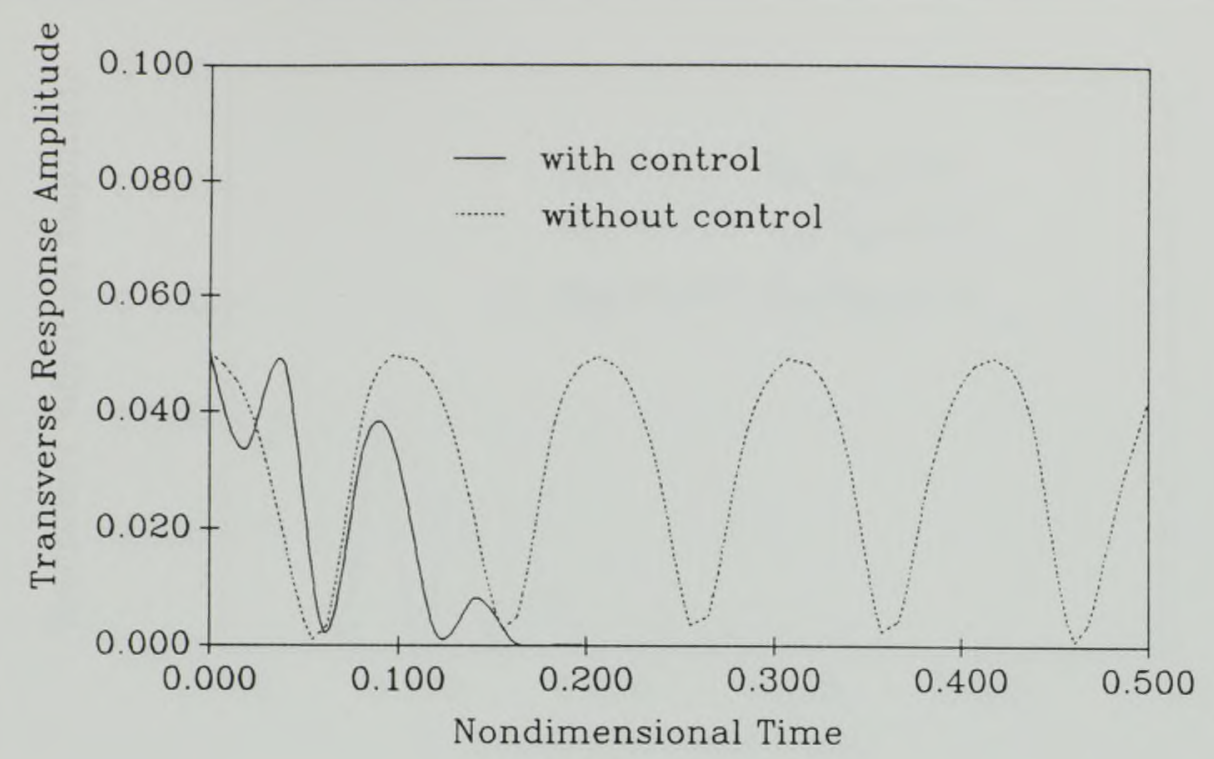

Figure 3.8 Comparison of results with and without active control

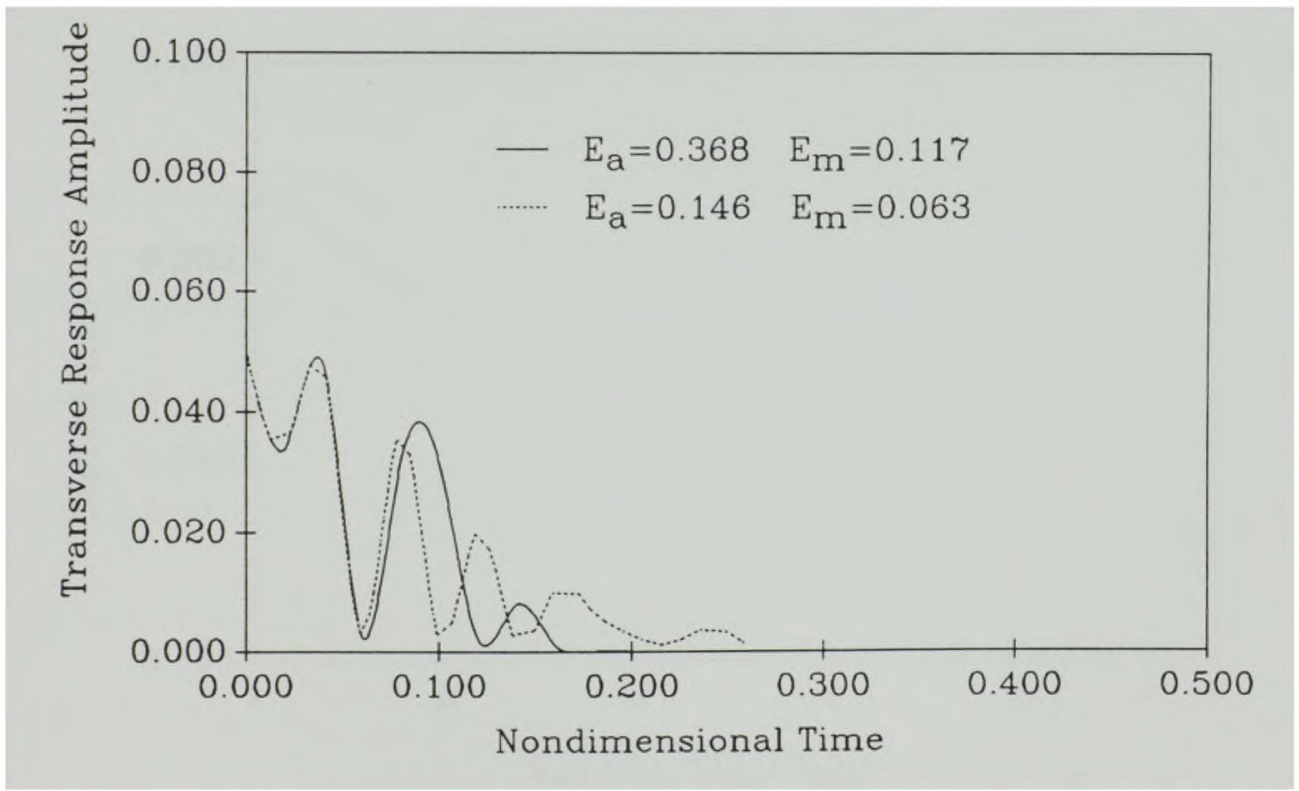

Figure 3.9 Control results for different SMA materials 


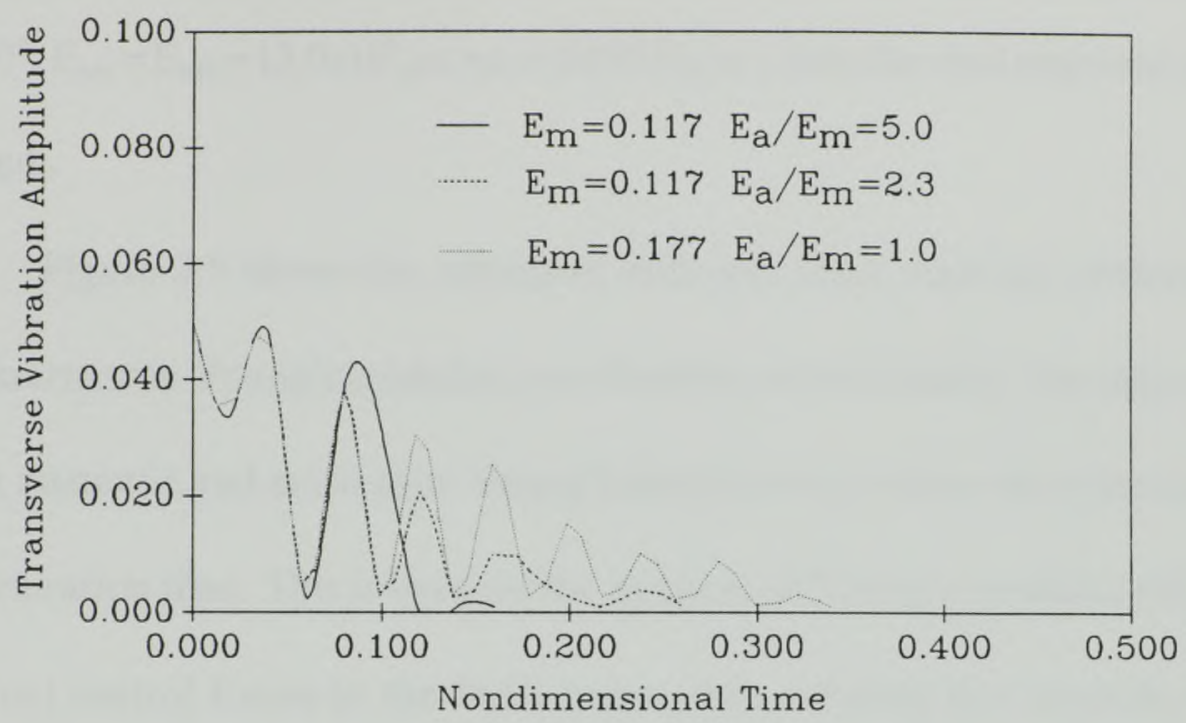

Figure 3.10 Control results for different SMA Young's modulus ratio

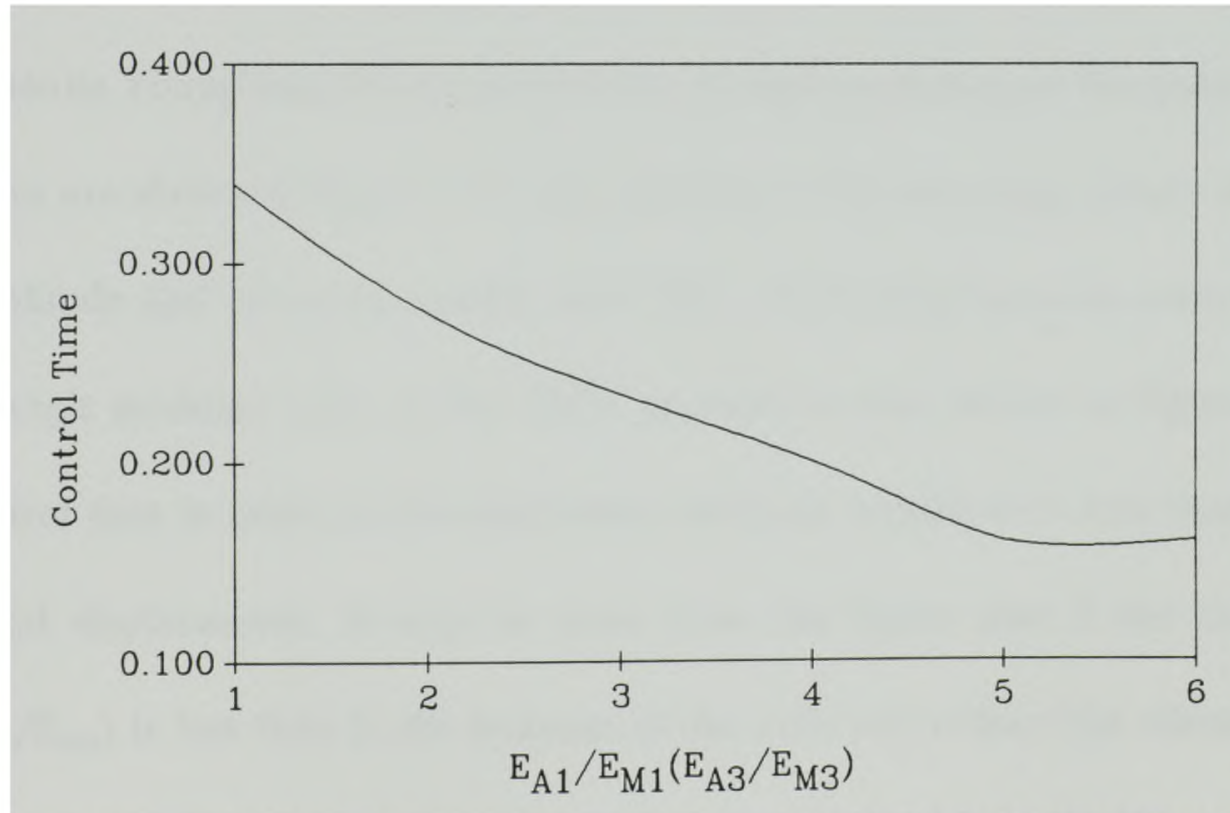

Figure 3.11 Vibration control time vs. SMA Young's modulus ratio 
of no control. If we consider a real beam with the following parameters: $L=500 \mathrm{~mm}$, $\mathrm{h}_{2}=10 \mathrm{~mm}, \mathrm{H}_{1}=\mathrm{h}_{1} / \mathrm{h}_{2}=0.1, \mathrm{H}_{3}=\mathrm{h}_{3} / \mathrm{h}_{1}, \mathrm{E}_{2}=20.6 \times 10^{10} \mathrm{~N} / \mathrm{m}^{2}, \rho_{2}=7800 \mathrm{Kg} / \mathrm{m}^{3}, \mathrm{E}_{\mathrm{Al}}=\mathrm{E}_{\mathrm{A} 3}=$ $30 \times 10^{9}, \mathrm{E}_{\mathrm{M} 1}=\mathrm{E}_{\mathrm{M} 3}=13.0 \mathrm{x} 10^{9}, \rho_{1}=\rho_{3}=6400 \mathrm{Kg} / \mathrm{m}^{3}$, then the real response time $\mathrm{t}=1.542$ second.

Figure 3.9 shows the effects of different SMA material (different austenite and martensite Young's modulus) on vibration control results. The selection of larger SMA austenite and martensite Young's modulus may reduce the vibration amplitude and vibration time. This is because the increase of Young's modulus will increase the internal control forces in the SMA layers, thus reducing the vibration control time and amplitude.

The effects of Young's modulus ratio of the same SMA material (the ratio of austenite Young's modulus to martensite Young's modulus) on the system frequency factor are shown in Figure 3.10. The increase of the ratio may reduce the vibration amplitude and vibration control time. The relationship between control time and Young's modulus ratio of the SMA material is also shown in Figure 3.11. The control time is taken as the time when vibration amplitude is less than $1 \%$ of the initial displacement. It may be seen from the figure that if the ratio $\mathrm{E}_{\mathrm{A} 1} / \mathrm{E}_{\mathrm{M1}}$ $\left(E_{A} / E_{M 3}\right)$ is less than 5 , the increase of the ratio will reduce the vibration control time greatly. But if the ratio is larger than 5 , the effect is negligible. 


\subsection{Conclusion}

In this chapter, the mathematical model of a flexible beam covered with shape memory alloy layers is presented. An active vibration control method for a flexible beam by means of SMA layers are discussed. Because of the heating and cooling process of using SMA material, the temperature effect is included in the analysis. It is shown that changes in the Young's modulus ratio and temperature of SMA layer will affect the natural frequency of the beam. It is noted that in the case of active vibration control, the response amplitude and vibration time will depend upon many factors such as the SMA material, Young's modulus ratio of the material (the ratio of austenite Young's modulus to martensite Young's modulus).

The application of such a model depends on the vibration control purposes. If it is used as an actuator, it could be used for low frequency vibration control (below $6 \mathrm{~Hz}$ ). If it is used for vibration tuning to avoid resonance, there is no restriction for the vibration frequency. 


\section{Chapter 4 Active and Passive Vibration Control}

\subsection{Governing equation of the beam}

In this section, the mathematical model of the flexible beam with constrained viscoelastic layer and shape memory alloy layer is presented. It is shown that change of the elastic modulus of the shape memory alloy layer will affect the system loss factor and resonance frequency. Also the recovery stress of the SMA layer functions like an excitation, thus changing the right side of the governing equation, i.e., adjusting the excitation. The effects of different parameters found in the analysis are discussed.

The beam with SMA \& viscoelastic treatments is shown in Figure 4.1. The upper side of the beam is bonded with a viscoelastic layer and then covered with a constraining layer. The lower side is bonded with a shape memory alloy layer. The following assumptions are made in the analysis: (1) the beam deflection is small and uniform across any section; (2) the primary beam and the constraining layer are assumed to be isotropic; (3) the longitudinal and rotatory inertia effects of the beam are neglected; (4) the damping layers carry shear, but no direct stress, and are assumed to be linear viscoelastic; (5) no slip occurs at the interface between the layers; and (6) the shape memory alloy layer is very thin; (7) the temperature effect will not be included temporarily. With these assumptions, the shear stress in the 


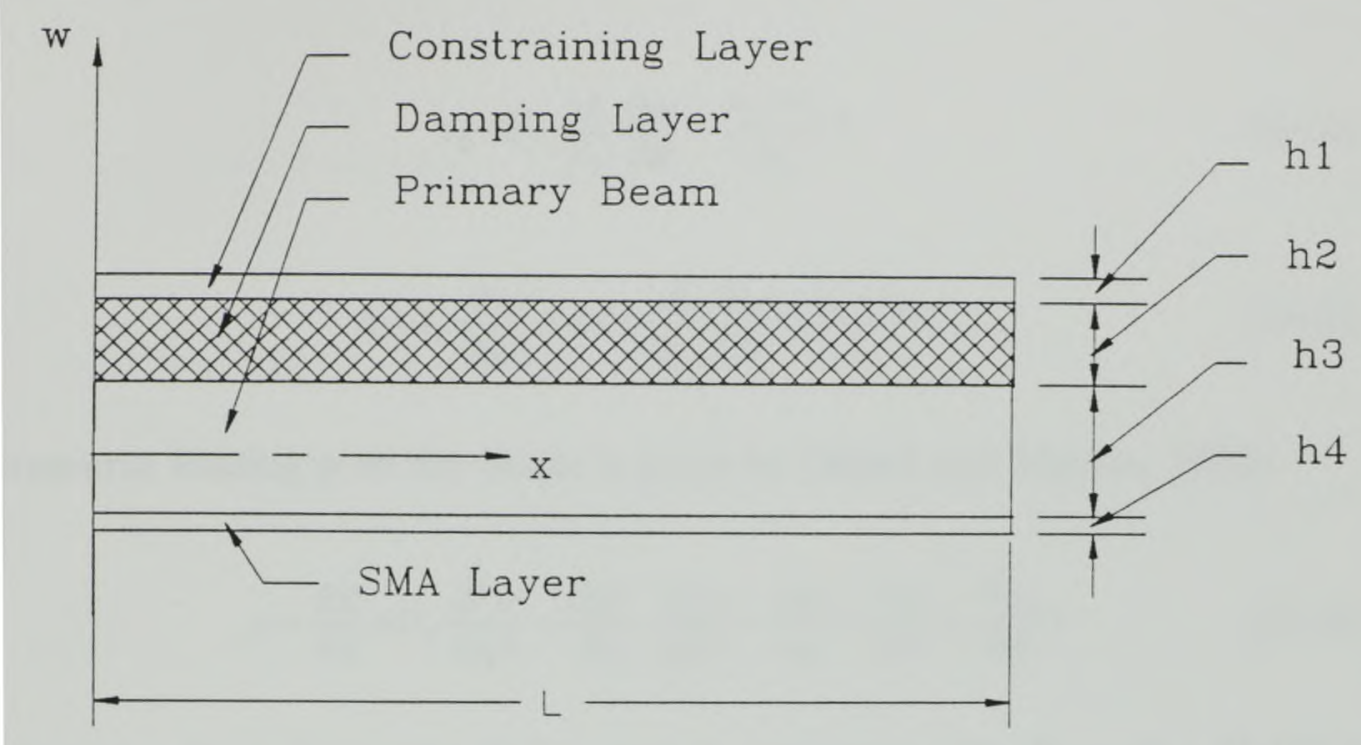

Figure 4.1 Beam with SMA and viscoelastic damping treatments

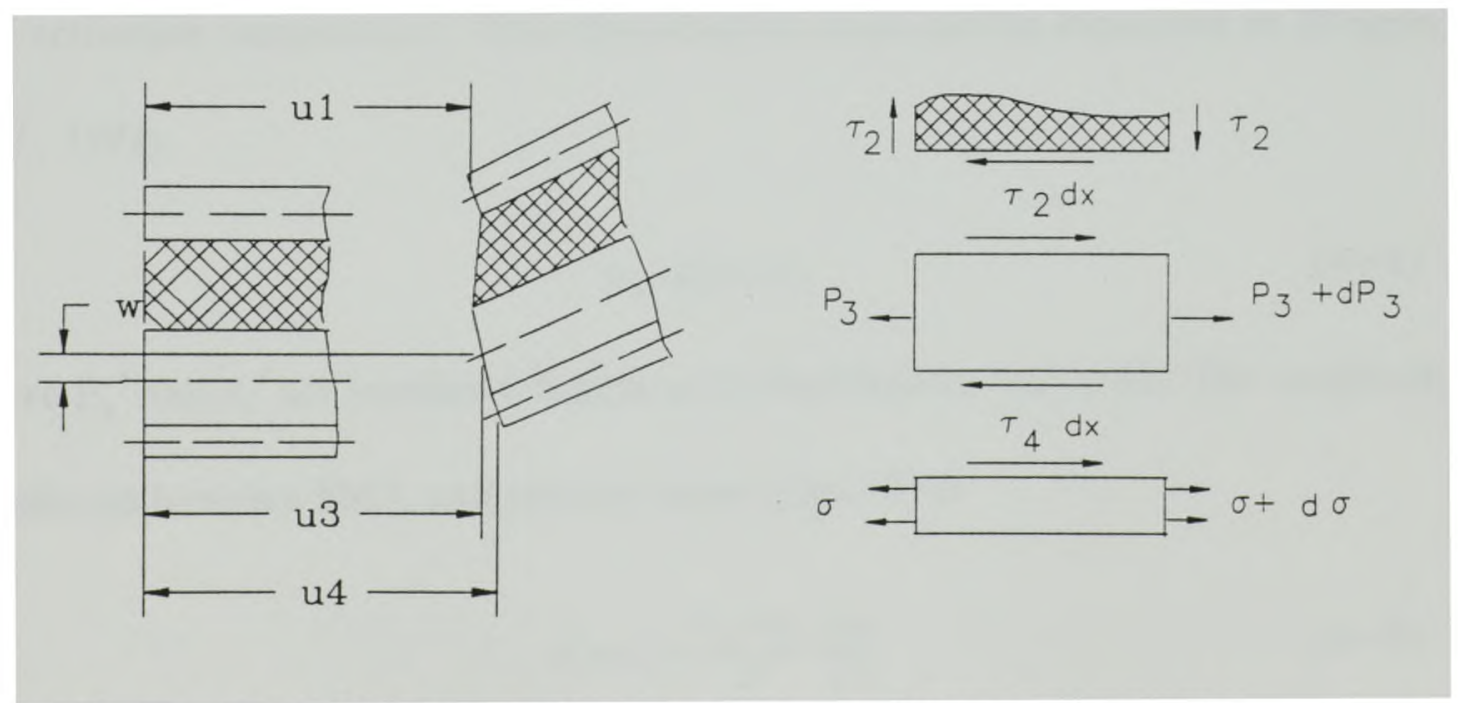

Figure 4.2 Forces and stresses on beam element 
damping layer and the total shear force on the section are given by (Mead and Markus, 1982).

$$
\begin{gathered}
\tau_{2}=G\left[\frac{d}{h_{2}} \frac{\partial W}{\partial x}+\frac{u_{1}-u_{3}}{h_{2}}\right] \\
S=D_{t} \frac{\partial^{3} w}{\partial x^{3}}-G d\left[\frac{d}{h_{2}} \frac{\partial w}{\partial x}+\frac{u_{1}-u_{3}}{h_{2}}\right]
\end{gathered}
$$

The transverse loading $\mathrm{p}$ on the beam is given by (Mead and Markus, 1982)

$$
p=\frac{\partial S}{\partial x}=D_{t} \frac{\partial^{4} W}{\partial x^{4}}-\frac{G d^{2}}{h_{2}} \frac{\partial^{2} w}{\partial x^{2}}-\frac{G d}{h_{2}}\left(\frac{\partial u_{1}}{\partial x}-\frac{\partial u_{3}}{\partial x}\right)
$$

For the SMA layer, let $E_{a}^{*}$, which is determined by Equation (3-18) by replacing subscript " 1 " or " 3 " by "a", be the elastic modulus at a given activation temperature $\mathrm{T}$, and $\sigma_{\mathrm{r}}^{*}$ be the recovery stress of the SMA layer at a specified strain and activation temperature. Then the actuator stress can be expressed as (Rogers, et al., 1991)

$$
\sigma_{a}=E_{a}^{*} \varepsilon+\sigma_{r}^{*}
$$

where $\mathrm{E}_{\mathrm{a}}^{*}$ and $\sigma_{\mathrm{r}}^{*}$ are nonlinear functions of temperature and strain. The continuity conditions between SMA and primary beam (layer 3 ) is

$$
u_{4}=u_{3}+\frac{h_{3}+h_{4}}{2} \frac{\partial W}{\partial x}
$$

Since the SMA layer is very thin, we assume that the stress is only a function of $x$ on 
the whole section. The resultant longitudinal force is zero, i.e.

$$
E_{1} h_{1} \frac{\partial u_{1}}{\partial x}+E_{3} h_{3} \frac{\partial u_{3}}{\partial x}+h_{4} E_{a}^{*} \frac{\partial u_{4}}{\partial x}+h_{4} \sigma_{r}^{*}=0
$$

From the force balance (Figure 4.2), we obtain

$$
\begin{gathered}
h_{4} E_{a}^{*} \frac{\partial^{2} u_{4}}{\partial x^{2}}+h_{4} \frac{\partial \sigma_{r}^{*}}{\partial x}=-\tau_{4}(x) \\
\frac{\partial P_{3}}{\partial x}=b\left(\tau_{4}-\tau_{2}\right)
\end{gathered}
$$

If expressed in terms of transverse displacement, $w(x, t)$, only, we obtain the differential equation of motion for the beam

$$
\begin{gathered}
\frac{D_{t} E_{1} h_{1} h_{2} C_{3}}{G} \frac{\partial^{6} w}{\partial x^{6}}-\left(D_{t} C_{1}+E_{1} h_{1} d^{2} C_{2} C_{3}+\frac{1}{2} E_{a}^{*} C_{1} d h_{1} h_{4}\right) \frac{\partial^{4} w}{\partial x^{4}} \\
=-C_{1} p+\frac{E_{1} h_{1} h_{2} C_{3}}{G} \frac{\partial^{2} p}{\partial x^{2}}+E_{1} h_{1} d h_{4} \sigma_{z}^{*}\left[\delta^{\prime}(x)-\delta^{\prime}(x-L)\right]
\end{gathered}
$$

where

$$
\begin{gathered}
C_{1}=E_{1} h_{1}+E_{3} h_{3}+E_{a}^{*} h_{4} \\
C_{2}=1-\frac{h_{3}+h_{4}}{2 d} \frac{E_{a}^{*} h_{4}}{E_{1} h_{1}} \\
C_{3}=E_{3} h_{3}+E_{a}^{*} h_{4}
\end{gathered}
$$

When the beam is under the action of transversely applied load, $q(x, t)$, the total inertia load is given by 
the whole section. The resultant longitudinal force is zero, i.e.

$$
E_{1} h_{1} \frac{\partial u_{1}}{\partial x}+E_{3} h_{3} \frac{\partial u_{3}}{\partial x}+h_{4} E_{a}^{*} \frac{\partial \dot{u}_{4}}{\partial x}+h_{4} \sigma_{r}^{*}=0
$$

From the force balance (Figure 4.2), we obtain

$$
\begin{gathered}
h_{4} E_{a}^{*} \frac{\partial^{2} u_{4}}{\partial x^{2}}+h_{4} \frac{\partial \sigma_{z}^{*}}{\partial x}=-\tau_{4}(x) \\
\frac{\partial P_{3}}{\partial x}=b\left(\tau_{4}-\tau_{2}\right)
\end{gathered}
$$

If expressed in terms of transverse displacement, $w(x, t)$, only, we obtain the differential equation of motion for the beam

$$
\begin{gathered}
\frac{D_{t} E_{1} h_{1} h_{2} C_{3}}{G} \frac{\partial^{6} w}{\partial x^{6}}-\left(D_{t} C_{1}+E_{1} h_{1} d^{2} C_{2} C_{3}+\frac{1}{2} E_{a}^{*} C_{1} d h_{1} h_{4}\right) \frac{\partial^{4} W}{\partial x^{4}} \\
=-C_{1} p+\frac{E_{1} h_{1} h_{2} C_{3}}{G} \frac{\partial^{2} p}{\partial x^{2}}+E_{1} h_{1} d h_{4} \sigma_{x}^{*}\left[\delta^{\prime}(x)-\delta^{\prime}(x-L)\right]
\end{gathered}
$$

where

$$
\begin{gathered}
C_{1}=E_{1} h_{1}+E_{3} h_{3}+E_{a}^{*} h_{4} \\
C_{2}=1-\frac{h_{3}+h_{4}}{2 d} \frac{E_{a}^{*} h_{4}}{E_{1} h_{1}} \\
C_{3}=E_{3} h_{3}+E_{a}^{*} h_{4}
\end{gathered}
$$

When the beam is under the action of transversely applied load, $q(x, t)$, the total inertia load is given by 


$$
p(x, t)=-m \frac{\partial^{2} w}{\partial t^{2}}+q(x, t)
$$

where $\mathrm{m}$ is the mass per unit length of the beam. Thus the differential equation for the forced vibration is given by

$$
\begin{aligned}
& \frac{D_{t} E_{1} h_{1} h_{2} C_{3}}{G} \frac{\partial^{6} W}{\partial x^{6}}-\left(D_{t} C_{1}+E_{1} h_{1} d^{2} C_{2} C_{3}+\frac{1}{2} E_{a}^{*} C_{1} d h_{1} h_{4}\right) \frac{\partial^{4} W}{\partial x^{4}} \\
& -m\left(-\frac{E_{1} h_{1} h_{2} C_{3}}{G} \frac{\partial^{4} W}{\partial x^{2} \partial t^{2}}+C_{1} \frac{\partial^{2} W}{\partial t^{2}}\right) \\
= & -C_{1} q(x, t)+\frac{E_{1} h_{1} h_{2} C_{3}}{G} \frac{\partial^{2} q}{\partial x^{2}}+E_{1} h_{1} d h_{4} \sigma_{r}^{*}\left[\delta^{\prime}(x)-\delta^{\prime}(x-L)\right]
\end{aligned}
$$

From the above equation, we know: (1) the recovery stress functions as an excitation to the system and we can change the excitation by adjusting the recovery stress in the SMA layer; and (2) the change of $E_{a}^{*}$ in the SMA layer will change the value of the natural frequency and system loss factor since the $C_{i}$ s are functions of $E_{a}^{*}$. Hence, even though the damping layer and constraining layer can not be changed after construction, we can still adjust the natural frequency and system loss factor to avoid resonance or to suppress the vibration of the beam. We call this kind of damping smart damping treatment(SDT). The boundary conditions for simply supported beam at $\mathrm{x}=0$ and $\mathrm{x}=\mathrm{L}$ are:

$$
\begin{aligned}
& w(0, t)=0, \quad \frac{\partial^{2} w(0, t)}{\partial x^{2}}=0, \quad \frac{\partial^{4} w(0, t)}{\partial x^{4}}=0 \\
& w(L, t)=0, \quad \frac{\partial^{2} w(L, t)}{\partial x^{2}}=0, \quad \frac{\partial^{4} w(L, t)}{\partial x^{4}}=0
\end{aligned}
$$




\subsection{Frequency and loss factor}

In order to discuss the effect of $E_{a}^{*}$ on the resonance frequency and loss factor of the beam, we seek the homogeneous form of equation (4-11). Assume the solution of the form

$$
w=B e^{j Q t} e^{k x}
$$

where $\Omega$ and $\mathrm{k}$ are the unknown complex natural frequency and characteristic values. Substituting equation (4-14) into the homogeneous form of equation (4-11) yields

$$
\begin{gathered}
\frac{C_{3} D_{t} E_{1} h_{1} h_{2}}{G} k^{6}-\left(D_{t} C_{1}+E_{1} h_{1} d^{2} C_{2} C_{3}+\frac{1}{2} E_{\mathrm{a}}^{*} C_{1} d h_{1} h_{4}\right) k^{4} \\
-m \Omega^{2}\left(\frac{E_{1} h_{1} h_{2} C_{3}}{G} k^{2}-C_{1}\right)=0
\end{gathered}
$$

The complex resonance frequency, $\Omega$, the associated real frequency, $\omega$, and the system loss factor of the beam, $\eta$, are related by

$$
\Omega^{2}=\omega^{2}(1+j \eta)
$$

For any $\Omega$, there are six roots, $\mathrm{k}_{1}, \mathrm{k}_{2}, \mathrm{k}_{3}, \mathrm{k}_{4}, \mathrm{k}_{5}, \mathrm{k}_{6}$ from equation (4-16). By writing the solution (4-14) in the following form

$$
W=\sum_{i=1}^{6} B_{i} e^{k_{1} x} e^{j \otimes t}
$$

The $B_{i} s(i=1,2, \ldots, 6)$ have to be determined from the boundary conditions. Substituting equations (4-17) into (4-12) and (4-13), we obtain six homogeneous 
equations involving the unknowns $B_{i}$ s. For nontrivial solutiol

determinant must be zero, i.e.

$$
\operatorname{det}(\Omega, k)=0
$$

Equations (4-15) and (4-18) are two nonlinear complex equations $f c$ and $\mathrm{k}$. A modified Muller's method was developed to find $\mathrm{k}$ and $\Omega$, thu resonance frequency and loss factor.

\subsection{Optimal Control}

From equation (4-11), the differential equations governing the motion of beam are found to be (with $q(x, t)=0)$ :

$$
\begin{gathered}
\frac{D_{t} E_{1} h_{1} h_{2} C_{3}}{G} \frac{\partial^{6} w}{\partial x^{6}}-\left[D_{t} C_{1}+E_{1} h_{1} d^{2} C_{2} C_{3}\right. \\
\left.\frac{1}{2} E_{a}^{*} C_{1} d h_{2} h_{4}\right] \frac{\partial^{4} w}{\partial x^{4}}+\frac{m}{b}\left(\frac{E_{1} h_{1} h_{2} C_{3}}{G} \frac{\partial^{4} w}{\partial x^{2} \partial t^{2}}-C_{1} \frac{\partial^{2} w}{\partial t^{2}}\right) \\
=E_{1} h_{1} d h_{4} \sigma_{r}^{*}\left[\delta^{\prime}(x)-\delta^{\prime}(x-L)\right]
\end{gathered}
$$

where $w(x, t)$ is the transverse displacement, $E_{a}{ }^{*}$ is the Young's modulus of the SMA layer and $\sigma_{r}^{*}$ is the recovery stress of the SMA layer. The definitions of the other parameters are given in the list of symbols. We can adjust the recovery stress in a desired way by changing the temperature, $T$, thus adjusting the excitation to minimize 


$$
\begin{array}{ll}
\bar{C}_{1}=1+\bar{E}_{1} H_{1}+\bar{E}_{a} H_{4} & \bar{C}_{2}=1-\frac{\left(1+H_{4}\right) \bar{E}_{a} H_{4}}{2 \bar{d} \bar{E}_{1} H_{1}} \\
\bar{C}_{3}=1+\bar{E}_{a} H_{4} & \bar{d}=H_{2}+\frac{1}{2}\left(1+H_{1}\right) \\
\bar{d}_{1}=1+H_{2}+\frac{1}{2}\left(H_{1}+H_{4}\right) & C_{4}=\left(1+\bar{E}_{1} H_{1}^{3}\right) \bar{E}_{1} H_{1} H_{2} H_{3}^{5} / \bar{G} \\
C_{5}=\left[\frac{1}{12}\left(1+\bar{E}_{1} H_{1}^{3}\right) \bar{C}_{1}+\bar{E}_{1} H_{1} \bar{d}^{2} \bar{C}_{2} \bar{C}_{3}+\frac{1}{2} \bar{E}_{a} \overline{C_{1}} \bar{d} H_{2} H_{4}\right] H_{3}^{3} \\
C_{6}=1+\bar{E}_{1} H_{1}+\bar{E}_{a} H_{4} & C_{7}=\bar{E}_{1} H_{1} H_{2} \bar{C}_{3} H_{3}^{3} / \bar{G}
\end{array}
$$

In the case of a simply supported beam, the boundary conditions at $\bar{x}=0$ and 1 are:

$$
\bar{w}(\bar{x}, \bar{t})=0, \quad \frac{\partial^{2} \bar{w}(\bar{x}, \bar{t})}{\partial \bar{x}^{2}}=0, \quad \frac{\partial^{4} \bar{w}(\bar{x}, \bar{t})}{\partial \bar{x}^{4}}=0
$$

The transverse displacement response of the beam is assumed to be of the form:

$$
\bar{w}(\bar{x}, \bar{t})=\sum_{i=1}^{\infty} T_{i}(\bar{t}) \Phi_{i}(\bar{x}),
$$

where $T_{i}(\tau)$ is the time dependent function to be determined and the eigenfunction of the beam $\Phi_{i}(\bar{x})$ is taken as $\sin \lambda_{i} \bar{x}$ to satisfy the boundary conditions. Substituting Equations (4-22) into (4-20) and using the orthogonal relationships yield:

$$
\ddot{T}_{i}(\mathcal{\tau})+A_{i} T_{i}(\tau)=D_{i} u(\tau),
$$

where 


$$
\begin{aligned}
& A_{i}=\frac{C_{4} \lambda_{i}^{6}-C_{5} \lambda_{i}^{4}}{C_{6}\left(C_{7} \lambda_{i}^{2}-\bar{C}_{1} H_{3}\right)} \\
& D_{i}=\frac{2 \lambda_{i}\left[1-(-1)^{i}\right]}{C_{7} \lambda_{i}^{2}-\bar{C}_{1} H_{3}} \\
& u(\bar{t})=\frac{\bar{E}_{1} H_{1} \bar{d} H_{4} H_{3}^{2}}{C_{6}} \bar{\sigma}
\end{aligned}
$$

Even though the solution is made up of infinite terms, only a finite number will have an appreciable effect on the displacement. If we assume a $2 n$-dimensional state variable vector to be:

$$
\dot{X}_{c}(\bar{t})=\left[T_{1}(\bar{t}), \ldots, T_{n}(\bar{t}) ; \dot{T}_{1}(\bar{t}), \ldots, \dot{T}_{n}(\bar{t})\right]^{T}
$$

where the superscript, $T$, denotes the transpose of the vector, eq. (4-23) may be written as:

$$
\dot{X}_{c}(\bar{t})=A_{c} X_{c}(\bar{t})+D_{c} u(\bar{t})
$$

where

$$
A_{c}=\left[\begin{array}{cc}
0 & I \\
-\delta_{i j} A_{i} & 0
\end{array}\right], \quad D_{c}=\left[\begin{array}{cc}
0 & 0 \\
0 & \delta_{i j} D_{i}
\end{array}\right] \text {. }
$$

$I$ is the identity matrix and $\delta_{i j}$ is the Kronecker delta. If a sensor is attached at $\bar{x}=$ $\bar{x}_{\xi}$, the corresponding deflection at that point is $\bar{w}_{\xi}\left(\bar{x}_{\xi} \bar{t}\right)$. The output is then given by:

$$
Y_{c}=d_{c} X_{c}(\tau)
$$

where $d_{c}$ is a constant vector and $d_{c}=\left[X_{1}\left(x_{\xi}\right), \ldots, X_{n}\left(X_{\xi}\right) ; 0, \ldots, 0\right]$. The performance 
index for a linear regulator problem is defined to be:

$$
J=\frac{1}{2} \int_{\bar{t}_{0}}^{\infty}\left(X_{c}^{T} Q X_{c}+u^{T} R u\right) d \bar{t}
$$

where $Q$ and $R$ are symmetric positive definite weighting matrices. An approximate choice of these matrices must be made to obtain "acceptable" levels of $x\left(\tau_{f}\right), x(\tau)$, and $u(\tau)$, where $\tau_{f}$ means terminal time. It is assumed that the desired optimal control law is:

$$
u^{*}(\tau)=-R^{-1} D_{c}{ }^{T} S(\tau) X^{*}(\tau)
$$

where $X^{*}(\tau)$ is the solution of the state equation with optimal control, $u^{*}$, as input, and $S(\tau)$ is a symmetric matrix to be determined by the following Riccati matrix equation:

$$
Q-S D_{c} R^{-1} D_{c}^{T}+A_{c}^{T} S+S A_{c}=\dot{S}
$$

Since we are interested in controlling the steady state vibration or controlling the structure over a longtime interval, $\dot{S}$ in Riccati equation is set to be zero.

\subsection{Temperature Effects}

\subsubsection{Temperature Distribution}

We will include the temperature effect in the analysis by relaxing the 


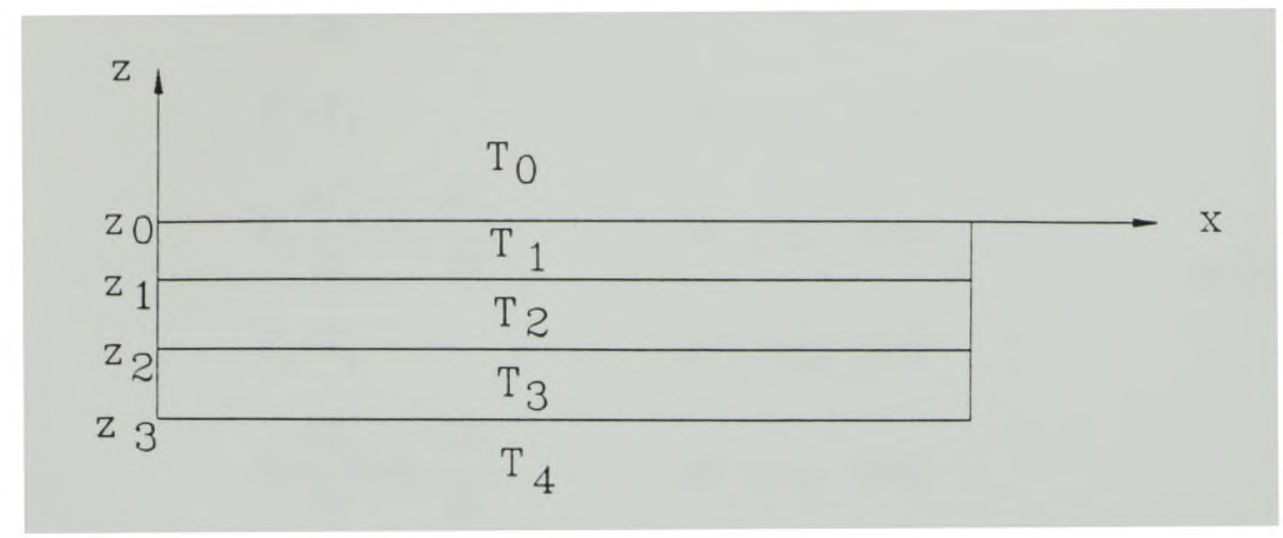

Figure 4.3 Temperature Distribution

assumption (7). As shown in the Figure 4.3, we assume that the temperature at the interface between the primary beam and SMA layer to be $T_{4}$ and the environmental temperature to $T_{0}$. Neglecting the longitudinal heat conduction, the temperature distribution inside the beam section is determined by solving the one-dimensional unsteady heat conduction problem

$$
\frac{\partial T_{i}}{\partial t}=\alpha_{T i} \frac{\partial^{2} T_{i}}{\partial z} \quad z_{i-1}<z<z_{i}, \quad t>0 \quad(i=1,2,3)
$$

subject to the boundary conditions: 


$$
\begin{array}{ll}
k_{1} \frac{\partial T_{1}}{\partial z}+\xi_{1} T_{1}=\xi_{1} T_{0} & \text { at } \quad z=z_{0} \quad t>0 \\
T_{1}=T_{2} & \text { at } \quad z=z_{1} \quad t>0 \\
k_{1} \frac{\partial T_{1}}{\partial z}=k_{2} \frac{\partial T_{2}}{\partial z} & \text { at } \quad z=z_{1} \quad t>0 \\
T_{2}=T_{3} & \text { at } \quad z=z_{2} \quad t>0 \\
k_{2} \frac{\partial T_{2}}{\partial z}=k_{3} \frac{\partial T_{3}}{\partial z} & \text { at } \quad z=z_{2} \quad t>0 \\
T_{3}=T_{4} & \text { at } \quad z=z_{3} \quad t>0
\end{array}
$$

We assume that the constraining layer, viscoelastic layer and primary beam are at $T_{0}$ initially when the SMA layer is "turned on" by having its temperature jumping instantaneously to $T_{4}$; thus:

$$
\begin{array}{lll}
T_{1}=T_{0} & \text { for } t=0 & z_{0}<z<z_{1} \\
T_{2}=T_{0} & \text { for } t=0 & z_{1}<z<z_{2} \\
T_{3}=T_{0} & \text { for } t=0 & z_{2}<z<z_{3}
\end{array}
$$

where $\mathrm{T}_{\mathrm{i}}, \mathrm{k}_{\mathrm{i}}, \alpha_{\mathrm{Ti}}(\mathrm{i}=1,2,3)$ are the temperature, material thermal conductivity and thermal diffusivity of $i$-th layer, respectively; $z_{\mathrm{i}}(\mathrm{i}=0,1,2,3)$ are the coordinates in the $\mathrm{z}$ direction; and $\xi_{1}$ is the convection heat transfer coefficient of the constraining layer. The temperatures $T_{i}(i=1,2,3)$ can be determined by solving Equations (4-30), (431a) and (4-31b). Introducing the following parameters: 


$$
\begin{array}{ll}
a_{0}=\left(k_{2}-k_{1}\right) z_{1}+k_{1} z_{2} & a_{1}=k_{2} / a_{0} \\
a_{2}=k_{1} / a_{0} & a_{3}=k_{1} k_{2} / a_{0} k_{3} \\
b_{1}=k_{1} k_{2} / \xi_{1} a_{0} & b_{2}=z_{1}\left(k_{2}-k_{1}\right) / a_{0} \\
b_{3}=1-k_{2} k_{1} z_{3} / k_{3} a_{0} &
\end{array}
$$

and functions:

$$
\begin{aligned}
& \left.\begin{array}{l}
\phi_{1}(z)=a_{1} z+b_{1} \\
\phi_{2}(z)=a_{2} z+b_{2} \\
\phi_{3}(z)=a_{3} z+b_{3}
\end{array}\right\} \\
& \varphi_{1 n}(z)=\cos \frac{\beta_{n} z}{\sqrt{ }^{\alpha_{T 1}}}+B_{1 n} \sin \frac{\beta_{n} z}{\sqrt{ }^{\alpha_{T 1}}} \\
& \varphi_{2 n}(z)=A_{2 n} \cos \frac{\beta_{n} z}{\sqrt{\alpha_{T 2}}}+B_{2 n} \sin \frac{\beta_{n} z}{\sqrt{\alpha_{T 2}}} \\
& \varphi_{3 n}(z)=A_{3 n} \cos \frac{\beta_{n} z}{\sqrt{\alpha}^{\alpha_{T 3}}}+B_{3 n} \sin \frac{\beta_{n} z}{\sqrt{\alpha_{T 3}}}
\end{aligned}
$$

where $\beta_{\mathrm{n}}$ is determined by the following characteristic equation for the heat transfer problem (In the equation, $\bar{\beta}_{\mathrm{ni}}=\beta_{\mathrm{n}} / \sqrt{ } \alpha_{\mathrm{Ti}}, \overline{\mathrm{k}}_{\mathrm{i}}=\mathrm{k}_{\mathrm{i}} / \sqrt{ } \alpha_{\mathrm{Ti}}, \mathrm{i}=1,2,3$ ). 







$$
\{C\}=\left\{\begin{array}{ccccc}
k_{1} \bar{\beta}_{n 1} & 0 & 0 & 0 & 0 \\
\sin \bar{\beta}_{n z} z_{1} & -\cos \bar{\beta}_{n 2} z_{1} & -\sin \bar{\beta}_{n z} z_{1} & 0 & 0 \\
\bar{k}_{1} \cos \bar{\beta}_{n z} z_{1} & \bar{k}_{2} \sin \bar{\beta}_{n z} z 1 & -\bar{k}_{2} \cos \bar{\beta}_{n z} z_{1} & 0 & 0 \\
0 & \cos \bar{\beta}_{n} z_{2} & \sin \bar{\beta}_{n} z_{2} & -\cos \bar{\beta}_{n 3} z_{2} & -\sin \bar{\beta}_{n z} z_{2} \\
0 & -\bar{k}_{2} \sin \bar{\beta}_{n} z_{2} & \bar{k}_{2} \cos \bar{\beta}_{n z} z_{2} & \bar{k}_{3} \sin \bar{\beta}_{n 3} z_{3} & -\bar{k}_{3} \cos \bar{\beta}_{n 3} z_{2}
\end{array}\right\}
$$

The heat distribution in each layer is found to be:

$$
T_{i}(z, t)=\psi_{i}(z, t)+\phi_{i}(z)\left(T_{4}-T_{0}\right)+T_{0} \quad(i=1,2,3)
$$

where

$$
\begin{gathered}
\psi_{i}(z, t)=\sum_{n=1}^{\infty} e^{-\beta^{2}, t} \frac{1}{N_{n}} \varphi_{i n}(z) \sum_{j=1}^{3} \frac{k_{j}}{\alpha_{j}} \int_{x_{j-1}}^{x_{j}} \varphi_{j n}\left(z^{\prime}\right) \phi_{j}\left(z^{\prime}\right)\left(T_{0}-T_{4}\right) d z^{\prime} \\
N_{n}=\sum_{j=1}^{3} \sum_{j} \int_{j} \int_{x_{j-1}}^{x_{j}} \varphi_{j n}^{2}\left(z^{\prime}\right) d z^{\prime}
\end{gathered}
$$




\subsubsection{Governing equations including temperature effect}

In general, the elastic modulus and loss factor of damping materials are functions of the frequency and temperature. The Young's modulus of the damping material may be expressed as (Jones, 1974):

$$
E=E_{d}(\omega, T)[1+j \eta(\omega, T)]
$$

where $\mathrm{E}_{\mathrm{d}}(w, \mathrm{~T})$ and $\eta(w, \mathrm{~T})$ are the real part of the complex Young's modulus and loss factor of damping material, respectively; and $w$ and $\mathrm{T}$ are the frequency and temperature, respectively. For most elastomeric materials in th erubbery and transition regions, $E_{d} \doteq 3 G_{d}$. The essential simplicity of the temperature-frequency equivalence principle lies in the assertion that the values of $E_{D}$ and $\eta$ at different frequencies and temperatures obey the following relationships (Jones, 1974):

$$
\left.\begin{array}{c}
E_{d}(\omega, T)=\left(T \rho / T \rho_{0}\right) E_{d}\left(\omega \alpha_{T}\right) \\
\eta(\omega, T)=\eta\left(\omega \alpha_{T}\right)
\end{array}\right\}
$$

where $\alpha_{\mathrm{T}}$ is a function of temperature known as the shift factor and $\mathrm{T}_{0}$ is a reference temperature. $\alpha_{\mathrm{T}}$ is given by (Jones, 1974)

$$
\alpha_{T} \doteq\left[1+\left(T-T_{0}\right) / 278\right]^{-14}
$$

In order to include the temperature effect, equation (4-4) and (4-6) are rewritten as: 


$$
\begin{gathered}
\sigma_{a}=E_{a}^{*}\left[\varepsilon+\alpha_{4}\left(T_{4}-T_{0}\right)\right]+\sigma_{r}^{*} \\
E_{1} h_{1}\left[\frac{\partial u_{1}}{\partial x}+\alpha_{1}\left(T_{1}-T_{0}\right)\right]+E_{3} h_{3}\left[\frac{\partial u_{3}}{\partial x}+\alpha_{3}\left(T_{3}-T_{0}\right)\right] \\
E_{a}^{*} h_{4}\left[\frac{\partial u_{4}}{\partial x}+\alpha_{4}\left(T_{4}-T_{0}\right)\right]+h_{4} \sigma_{r}^{*}=0
\end{gathered}
$$

From equations (4-1), (4-3), (4-5), (4-7), (4-8), (4-10), (4-39) and (4-40), we obtain the differential equation including the temperature effect as follows:

$$
\begin{gathered}
\frac{D E_{1} h_{1} h_{2} C_{3}}{G} \frac{\partial^{6} w}{\partial x^{6}}-\left[D_{t} C_{1}+E_{1} h_{1} d^{2} C_{3}+\frac{1}{2} E_{1} h_{1} E_{a}^{*} h_{4}\left(h_{3}+h_{4}\right) d\right] \frac{\partial^{4} w}{\partial x^{4}} \\
-m\left(-\frac{E_{1} h_{1} h_{2} C_{3}}{G} \frac{\partial^{4} w}{\partial x^{2} \partial t^{2}}+C_{1} \frac{\partial^{2} w}{\partial t^{2}}\right)=-C_{1} q(x, t)+\frac{E_{1} h_{1} h_{2} C_{3}}{G} \frac{\partial^{2} q}{\partial x^{2}} \\
-\left\{\alpha_{1} E_{1} h_{1} d C_{3}\left(T_{1}-T_{0}\right)-\alpha_{3} E_{3} h_{3} d C_{1}\left(T_{3}-T_{0}\right)\right. \\
\left.-E_{1} h_{1} h_{4} d\left[E_{a}^{*} \alpha_{4}\left(T_{4}-T_{0}\right)+\sigma_{r}^{*}\right]\right]\left[\delta^{\prime}(x)-\delta^{\prime}(x-L)\right]
\end{gathered}
$$

In the case of no temperature effect, by selecting the $\alpha_{\mathrm{i}}=0(i=1,3,4)$, equation (4-41) reduce to equation (4-11). In the case of no SMA layer and neglecting temperature effects, Equation (4-10) reduces to Equation (16) obtained by Mead and Markus (1969).

\subsubsection{Vibration Control}

In the case of no distribution load, let $\mathrm{q}(\mathrm{x}, \mathrm{t})$ in equation $(4-41)$ be zero. If we introduce the dimensionless parameters (4-20a) and coefficients (4-20b), Equation 
(4-41) can be rewritten as :

$$
\begin{gathered}
C_{4} \frac{\partial^{6} \bar{w}}{\partial \bar{x}_{6}}-C_{5} \frac{\partial^{4} \bar{w}}{\partial \bar{x}^{4}}+C_{6}\left(C_{7} \frac{\partial^{4} \bar{w}}{\partial \bar{x}^{2} \partial \bar{t}^{2}}-\bar{C}_{1} H_{3} \frac{\partial^{2} \bar{w}}{\partial \bar{t}^{2}}\right) \\
=\left\{-\alpha_{1} \bar{E}_{1} H_{1} \bar{d} \bar{C}_{3}\left(T_{1}-T_{0}\right)+\alpha_{3} \bar{E}_{3} H_{3} \bar{d} \bar{C}_{1}\left(T_{3}-T_{0}\right)\right. \\
\left.+\bar{E}_{1} H_{1} \bar{d} H_{4} H_{3}^{2}\left[\bar{E}_{a} \alpha_{4}\left(T_{4}-T_{0}\right)+\bar{\sigma}\right]\right\}\left[\delta^{\prime}(x)-\delta^{\prime}(x-1)\right]
\end{gathered}
$$

The transverse displacement of the beam may be assumed to be variable separable abd given by Equation (4-22). Substituting equation (4-22) into equation (4-42) and using the orthogonal relationships yield:

$$
\ddot{T}_{i}(\bar{t})+A_{i} T_{i}(\bar{t})=D_{i} u(\bar{t})
$$

where

$$
\left.\begin{array}{l}
A_{i}=\frac{C_{4} \lambda_{i}^{6}-C_{5} \lambda_{i}^{4}}{C_{6}\left(C_{7} \lambda_{i}^{2}-\bar{C}_{1} H_{3}\right)} \\
D_{i}=\frac{2 \lambda_{i}\left[1-(-1)^{i}\right]}{C_{f} \lambda_{i}^{2}-\bar{C}_{1} H_{3}} \\
u_{i}(\bar{t})=\frac{-\alpha_{1} \bar{E}_{1} H_{1} \bar{d} \bar{C}_{3} \bar{T}_{1}+\alpha_{3} \bar{E}_{3} H_{3} \bar{d} \bar{C}_{1} \bar{T}_{3}+\bar{E}_{1} H_{1} \bar{d} H_{4} H_{3}^{2}\left[\alpha_{4} \bar{E}_{a} \bar{T}_{4}+\bar{\sigma}\right]}{C_{6}} \\
\bar{T}_{i}=T_{i}-T_{0} \quad(i=1,3,4)
\end{array}\right\}
$$

if we use the coefficients defined by (4-44), equations (4-24)- (4-29) may still be used to solve the control problem in this case. 


\subsection{Numerical Results}

The input parameters, unless stated otherwise, were:

$$
\begin{gathered}
\mathrm{h}_{3} / \mathrm{L}=0.02, \mathrm{~h}_{1} / \mathrm{h}_{3}=\mathrm{h}_{2} / \mathrm{h}_{3}=1.0, \mathrm{~h}_{4} / \mathrm{h}_{3}=0.1, \mathrm{E}_{1} / \mathrm{E}_{3}=1.0, \mathrm{E}_{\mathrm{Aa}} / \mathrm{E}_{3}=0.368, \mathrm{E}_{\mathrm{Ma}^{2}} / \mathrm{E}_{3}=0.117, \\
\mathrm{Mf}=5.0{ }^{\circ} \mathrm{C}, \mathrm{M}_{\mathrm{s}}=23.00^{\mathrm{C}}, \mathrm{A}_{\mathrm{s}}=29.0{ }^{\circ} \mathrm{C}, \mathrm{A}_{\mathrm{f}}=51.0{ }^{\circ} \mathrm{C}, \mathrm{T}_{0}=25.0{ }^{\circ} \mathrm{C}, \mathrm{G} / \mathrm{E}_{3}=0.05, \\
\rho_{1}=\rho_{3}=7800 \mathrm{Kg} / \mathrm{m}^{3}, \rho_{2}=3140 \mathrm{Kg} / \mathrm{m}^{3}, \mathrm{E}_{1}=\mathrm{E}_{3}=20.6 \mathrm{~N} / \mathrm{m}^{2}, \xi_{1}=100 \mathrm{~J} /\left(\mathrm{hr} \cdot \mathrm{m}^{2} .^{\circ} \mathrm{C}\right), \\
\alpha_{\mathrm{T} 1}=\alpha_{\mathrm{T} 3}=0.042 \mathrm{~m}^{2} / \mathrm{hr}, \alpha_{\mathrm{T} 2}=0.35 \mathrm{~m} 2 / \mathrm{hr}, \mathrm{L}=0.5 \mathrm{~m} .
\end{gathered}
$$

\subsubsection{Frequency and Loss Factor}

Figure 4.4 shows the effects of SMA layer temperature to system frequency factor for both the first and second modes. If the temperature of the SMA layer is less than the martensite start temperature, the increase of the temperature will decrease the system frequency factor. Beginning at the austenite start temperature, the temperature increase of SMA layer will increase the frequency factor. For the temperature above the austenite finish temperature, the increase of SMA temperature will decrease the frequency factor. This is because temperature increase will generally decrease the frequency factor. But beginning at the austenite start temperature, the phase transformation due to temperature increase will increase the Young's modulus of SMA material, thus causing the increase of the frequency factor. After the austenite finish temperature, no further increase of Young's modulus will occur. So the frequency factor will decrease with increase of SMA layer temperature. 


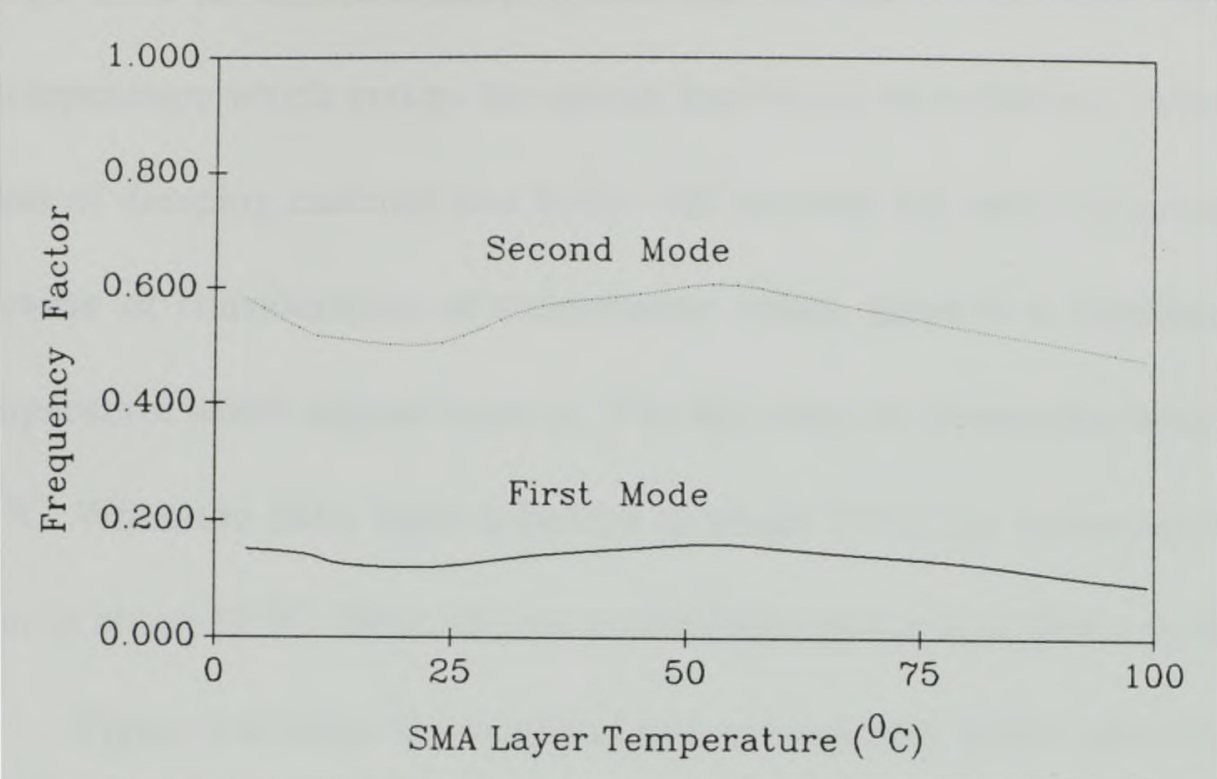

Figure 4.4 SMA layer temperature vs. system frequency factor

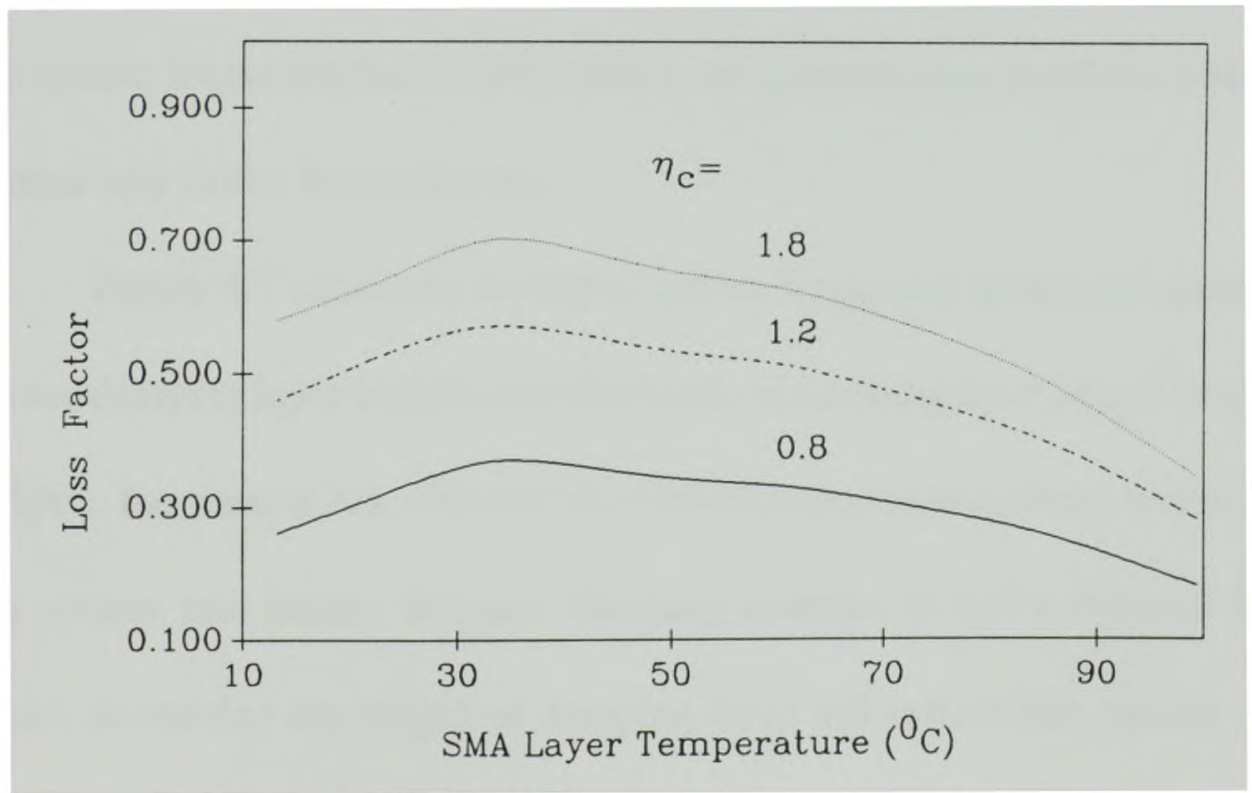

Figure 4.5 SMA layer temperature vs. system loss factor 
Figure 4.5 shows the result of system loss factor versus SMA layer temperature for different damping material loss factors. It is noted that there exists a temperature which makes the system loss factor be maximum. Also increase the value of damping material loss factor will increase the value of system loss factor. Because of consideration of temperature effect, there is a maximum loss factor temperature which depend upon $\eta_{\mathrm{c}}$. For this case, we choose the temperature to be $30{ }^{\circ} \mathrm{C}$. When the SMA layer is heated to about $37^{\circ} \mathrm{C}$, the temperature in damping layer is about $30^{\circ} \mathrm{C}$. Thus, we see a maximum system loss factor in the figure.

Figure 4-6 shows the results of system frequency factor and loss factor versus shear modulus ratio of damping layer (the ratio of damping layer shear modulus to Young's modulus of the primary beam). Increase of the shear modulus will increase the system frequency factor. But there is an optimal shear modulus which makes the system loss factor be maximum.

Figure 4-7 shows the results of system frequency factor and system loss factor versus damping layer height ratio (the ratio of damping layer height to primary beam height). Increase of the ratio will decrease the system frequency factor, but increase the system loss factor. Because damping material is soft compared with primary beam, increasing the height of damping layer will reduce the rigidity of the beam, thus decreasing the system frequency factor. 




Figure 4.6 Shear Modulus ratio vs. system frequency and loss factor

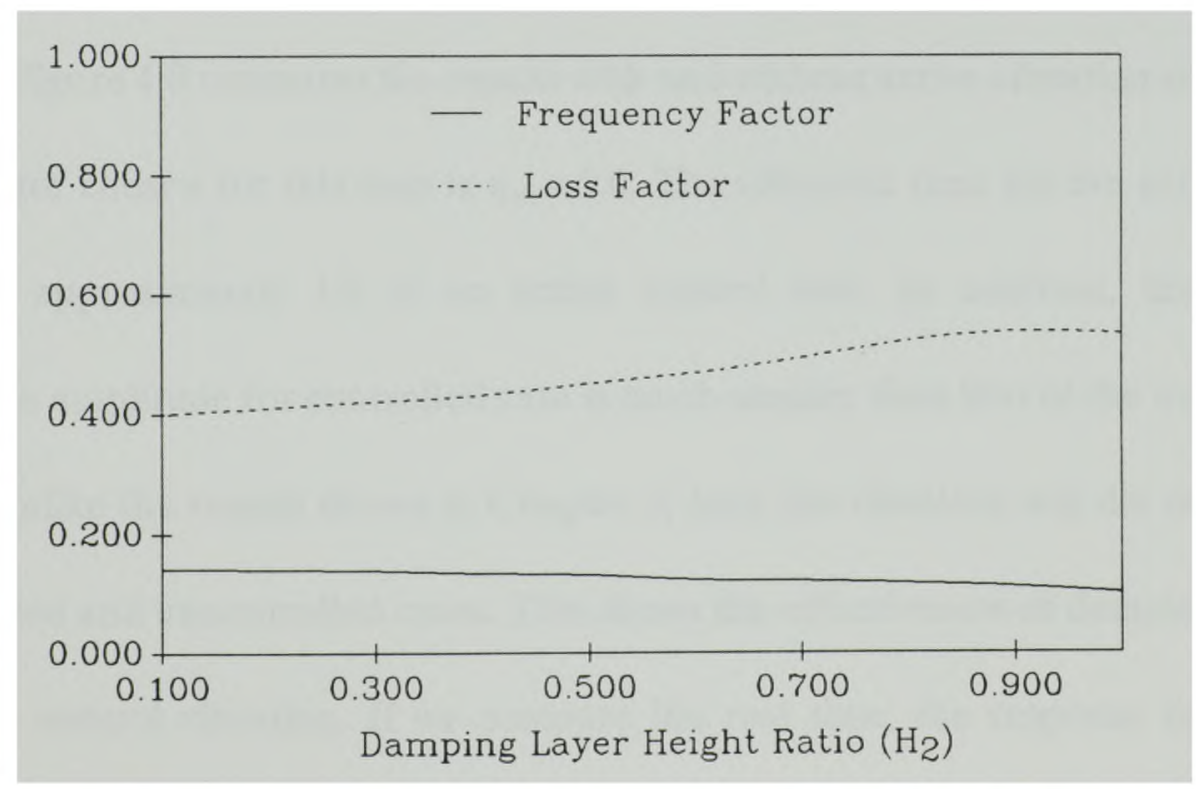

Figure 4.7 Damping layer height ratio vs. system frequency and loss factor 


\subsubsection{Control Results}

Figure $4.8-4.10$ shows the results of vibration control for different cases. I the figures, the horizontal axis represents nondimensional time while the vertical axis represents nondimensional displacement amplitude. The initial conditions chosen for the problems are:

$$
\bar{w}(\bar{x}, 0)=0.05, \quad \frac{\partial \bar{w}(\bar{x}, 0)}{\partial \bar{t}}=0
$$

The problem is solved iteratively. Given a time increment and the initial conditions, we try to obtain response displacement and velocity. Then using the solution as new initial condition, we move to next time step. We continue the process until the displacement is less than $1 / 100$ of initial value.

Figure 4.8 compares the results with and without active vibration control. The loss factor chosen for this case is $\eta_{\mathrm{cm}}=1.6$. The vibration time for the active control case is approximately $1 / 3$ of no active control case. In addition, the vibration response amplitude for controlled case is much smaller than that of the uncontrolled case. Unlike the results shown in Chapter 3, here the vibration will die out both for controlled and uncontrolled cases. This shows the effectiveness of damping material used to control vibration. If we compare the real time, the response time for the controlled case is $\mathbf{t}=3.24$ second; but the response time for the uncontrolled case is $\mathrm{t}=7.85$ second 


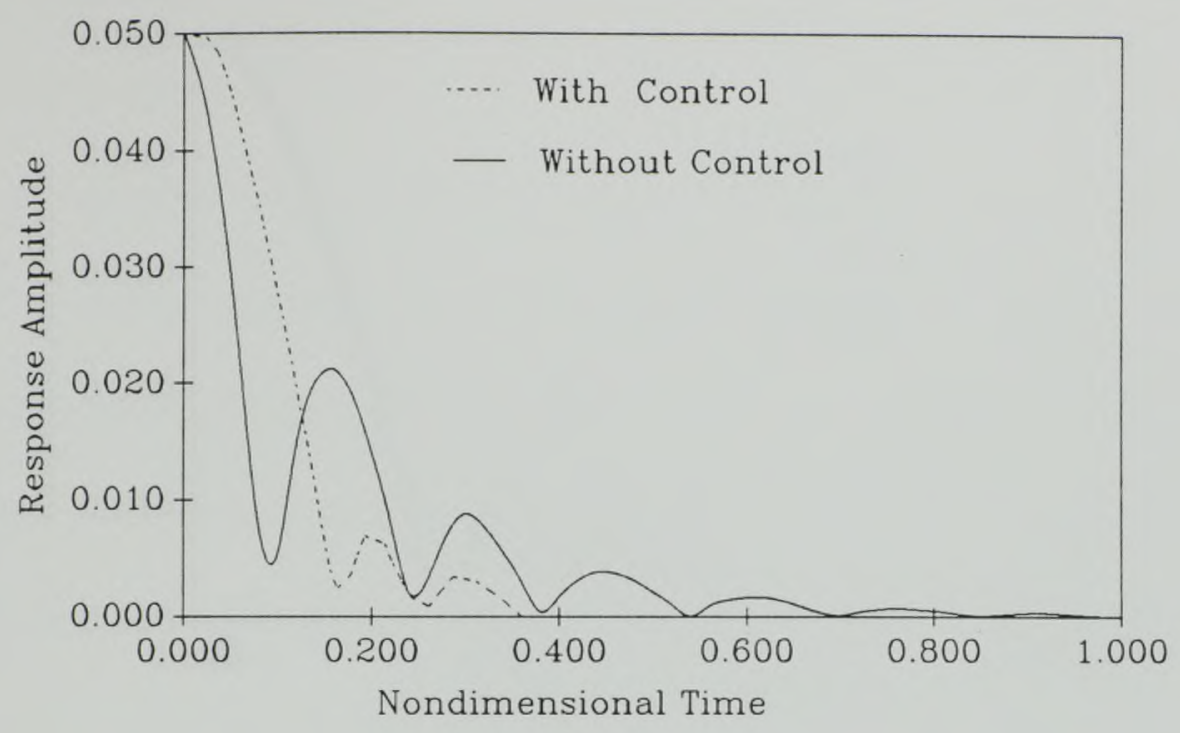

Figure 4.8 Comparison of results with and without active vibration control

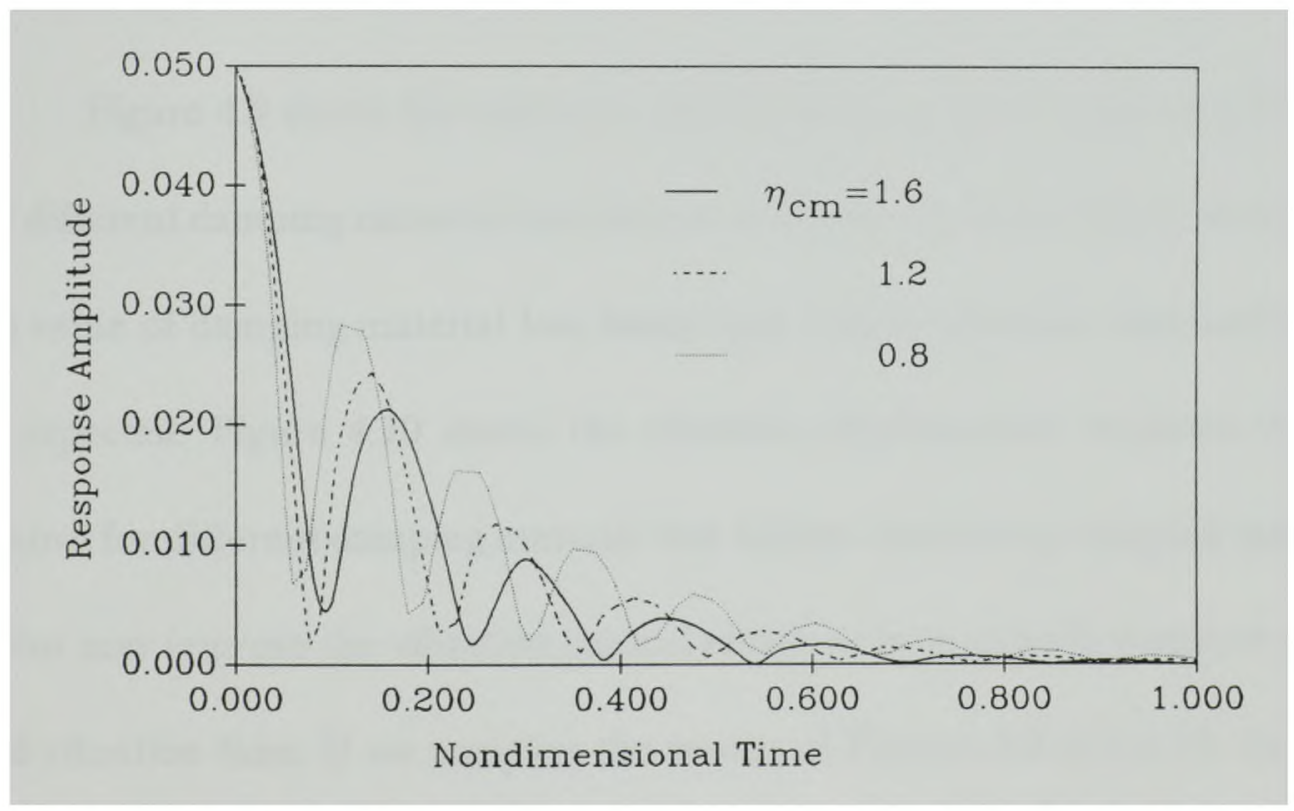

Figure 4.9 Vibration response for different damping material loss factor 




Figure 4.10 Active vibration control results for different damping loss factor

Figure 4.9 shows the vibration displacement response without active control for different damping material loss factors. It is noted from the figure that increasing the value of damping material loss factor may reduce vibration time and amplitude as expected. Figure 4.10 shows the vibration displacement response with active control for different damping material loss factors. Increasing damping material loss factor may improve the vibration control results in term of both response amplitude and vibration time. If we compare the results of Figures 4.9 and 4.10 , we note that active control case shows much better results (shows smaller vibration amplitude and less vibration time). 


\subsection{CONCLUSIONS}

Vibration control of a flexible beam using a constrained viscoelastic damping treatment and shape memory alloy is studied. The SMA layer is used as an actuator, which is capable of changing its elastic modulus and recovery stress. For the flexible vibration of beams with SMA and constrained damping treatment, the following conclusions may be obtained: 1) in the case of active vibration control, vibration will die out much quicker than in the no control case with the response amplitude being much smaller; 2) the introduction of damping treatment may improve the control results; 3) although there is a limitation in the control frequency range because of the heating and cooling process of the SMA actuators, this model may also be used in high frequency applications because of the employment of the constrained viscoelastic layer. 


\section{Chapter 5 Experiment}

In this chapter, the experimental study of the vibration characteristics for different systems will be presented. The experiments cover vibration characteristics of different constrained viscoelastic damping treatments with and without end mass.

\subsection{Experimental Schemes}

The experimental scheme for the analysis of dynamic characteristics is shown in Figure 5.1. It is mainly composed of the excitation system, flexible beam structure and data acquisition system. In order to measure the system characteristics (frequency, vibration mode and damping), we use the Bruel \& Kjar impact hammer type 8202 to excite the structure. The hammer is connected to a Bruel \& Kjar Line-Drive Amplifier type 2644, and then by means of a cable, the Line-Drive Amplifier is connected to the channel A Line-Drive Socket on the Bruel \& Kjar Dual Channel Signal Analyzer type 2034. The response accelerometer with line drive amplifier is mounted on the structure to be tested. The amplifier is connected to the

Channel B Line-Drive socket on the Dual Channel Signal Analyzer. As mentioned before, the experiments will be done for many different cases. The data are 
Computer with

STAR System
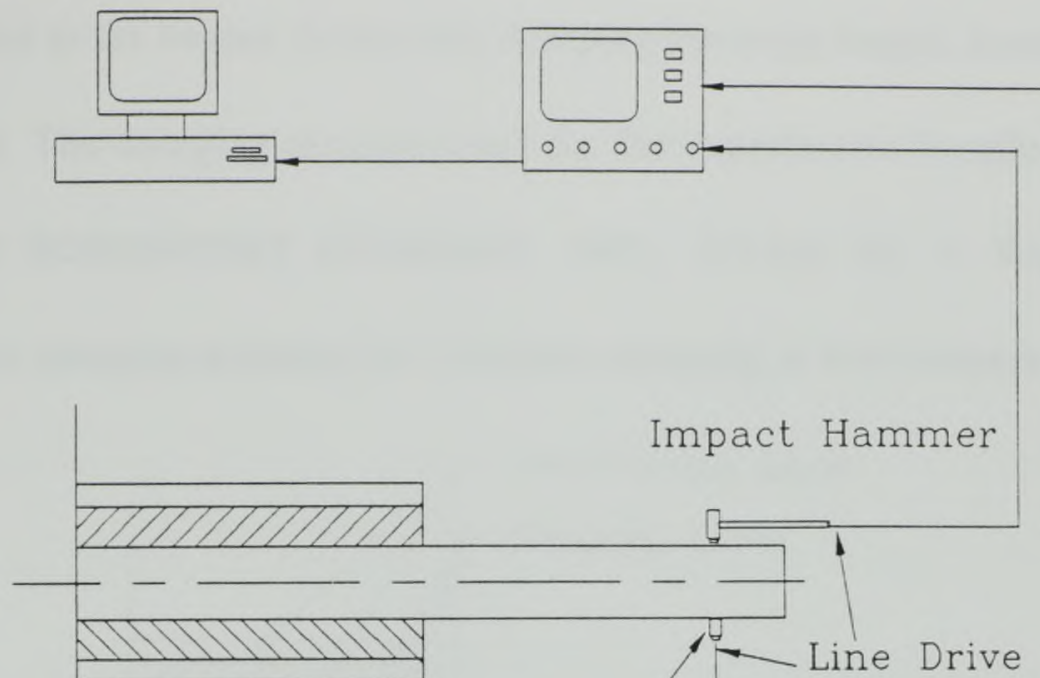

Accelerometer

Figure 5.1 Experimental Scheme

measured by the Dual Channel Signal Analyzer, and then analyzed. Also the data will be transferred to a PC computer with the StarStruct System which is a series of software products for testing and analyzing the dynamics of mechanical structures. The data transfer is done via the GPIB (IEEE 488) Interface Bus. 


\subsection{Vibration Experiments of Constrained Viscoelastic Damping Structure}

The damping treatment considered here is a constrained viscoelastic layer treatment applied to the beams. In the test, damping coverage length, location, etc., will be analyzed. The damping material used for the experiments is called DYAD 601 from THE SOUNDCOAT COMPANY, INC.. DYAD 601 is Soundcoat's constrained layer damping material for vibration damping at low temperatures.

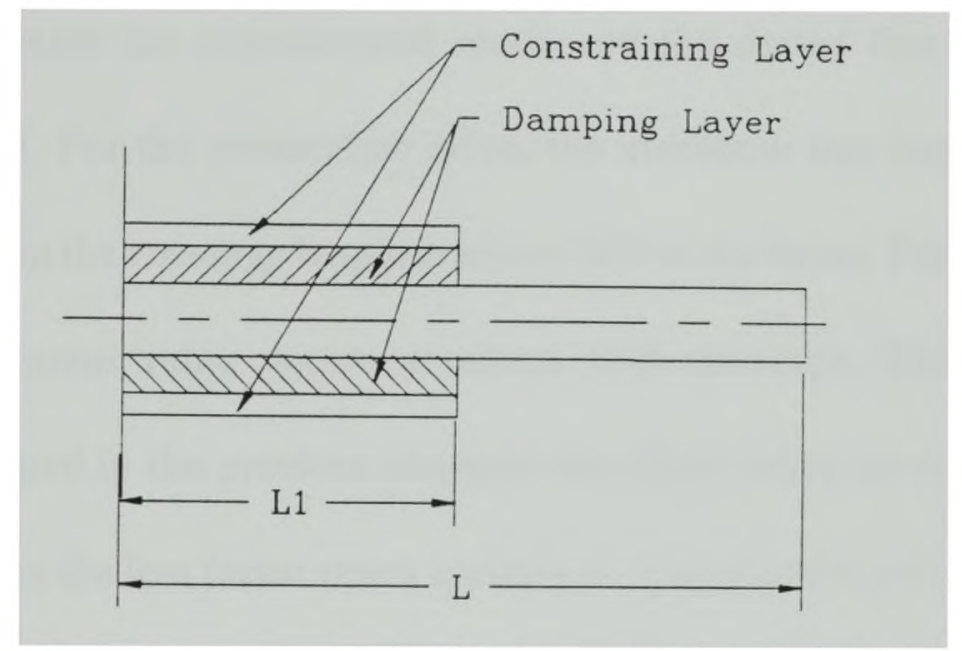

Figure 5.2 Attachment of damping layer and constraining layer

DYAD 601 provides maximum damping at about $50^{\circ} \mathrm{F}\left(10^{\circ} \mathrm{C}\right)$, but the material has a useful damping range from $-10^{\circ}$ to $100^{\circ} \mathrm{F}\left(-23^{\circ}\right.$ to $\left.38^{\circ} \mathrm{C}\right)$. The sample for the original structure is a steel beam with the dimension $342.9^{\mathrm{mm}}$ long $\mathrm{x} 31.75^{\mathrm{mm}}$ wide $\mathrm{x} 3.175^{\mathrm{mm}}$ high. The dimensions for the DYAD 601 damping material are $31.75^{\mathrm{mm}}$ wide $\times 0.6^{\mathrm{mm}}$ high with different length of coverage. The constraining layer is an aluminum sheet with dimensions $31.75^{\mathrm{mm}}$ wide $\times 1^{\mathrm{mm}}$ high with different length of coverage. The attachment of damping layer and constraining layer on the beam is shown in Figure 
shown in Figure 5.2. The length of coverage $\mathrm{L}_{1}$ is varied for different cases. We take the values to be $52.324 \mathrm{~mm}, 106.68 \mathrm{~mm}, 190.5 \mathrm{~mm}$ and $304.8 \mathrm{~mm}$, respectively. The experimental results for different coverage and end masses are shown in Figures 5.3 -

\section{7.}

Case 1 Coverage Length Effects

Figure 5.3 shows the results of system loss factor versus coverage ratio. The solid line represents the experimental result and the dotted line represents the theoretical result. For the parameters given, the maximum loss factor for the first mode occurs when the coverage length is about $70 \%$ of the beam. For the theoretical result, the maximum value occurs at about $60 \%$ coverage. This confirms the conclusion obtained in the previous chapters that there exists an optimal coverage ratio which makes the loss factor reach maximum. Also, the theoretical value of loss factor is smaller than the experimental result. The main reason is that in the experiments, we used the epoxy adhesives to connect the damping layers with beam and constraining layers. These adhesive layers would dissipate a certain amount of energy while such effects are not considered in the theoretical analysis. The error for system loss factor between theory and experiment is about $12 \%$.

The Effects of coverage length to the natural frequency of the system are shown in Figure 5.4. The solid line represents the experimental result of the first natural frequency, The dotted line represents the theoretical result. We note from 


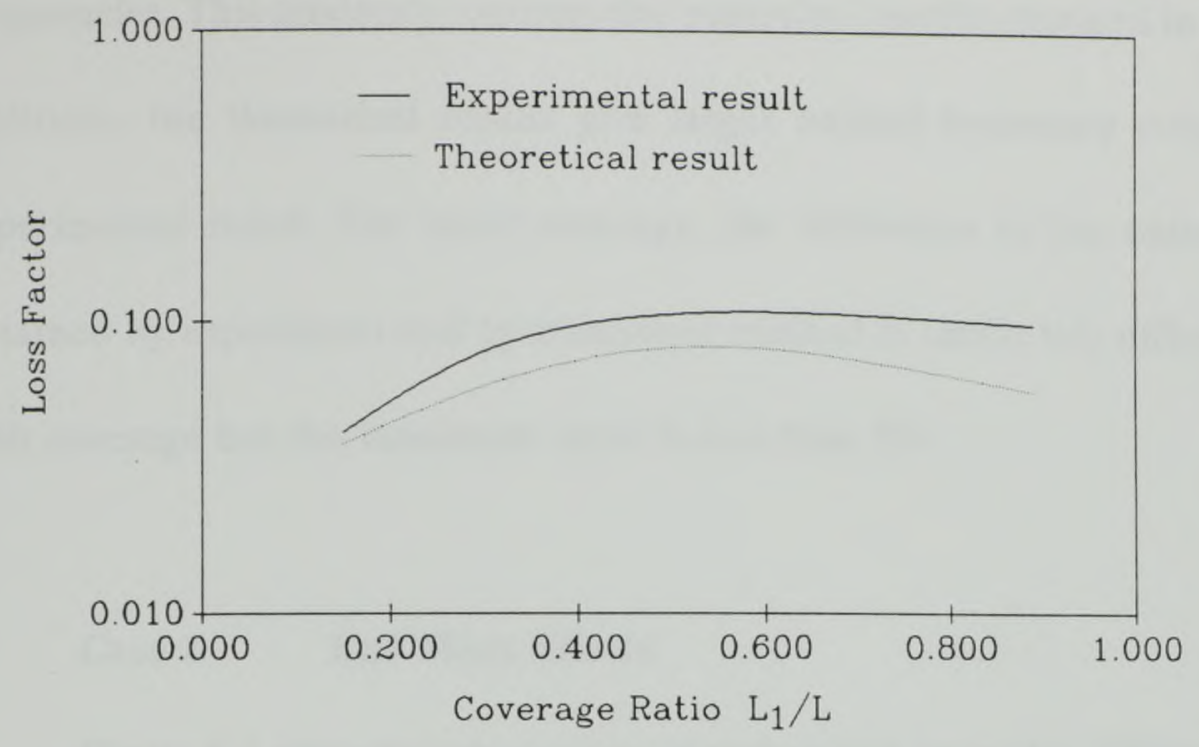

Figure 5.3 Coverage length ratio vs. system loss factor

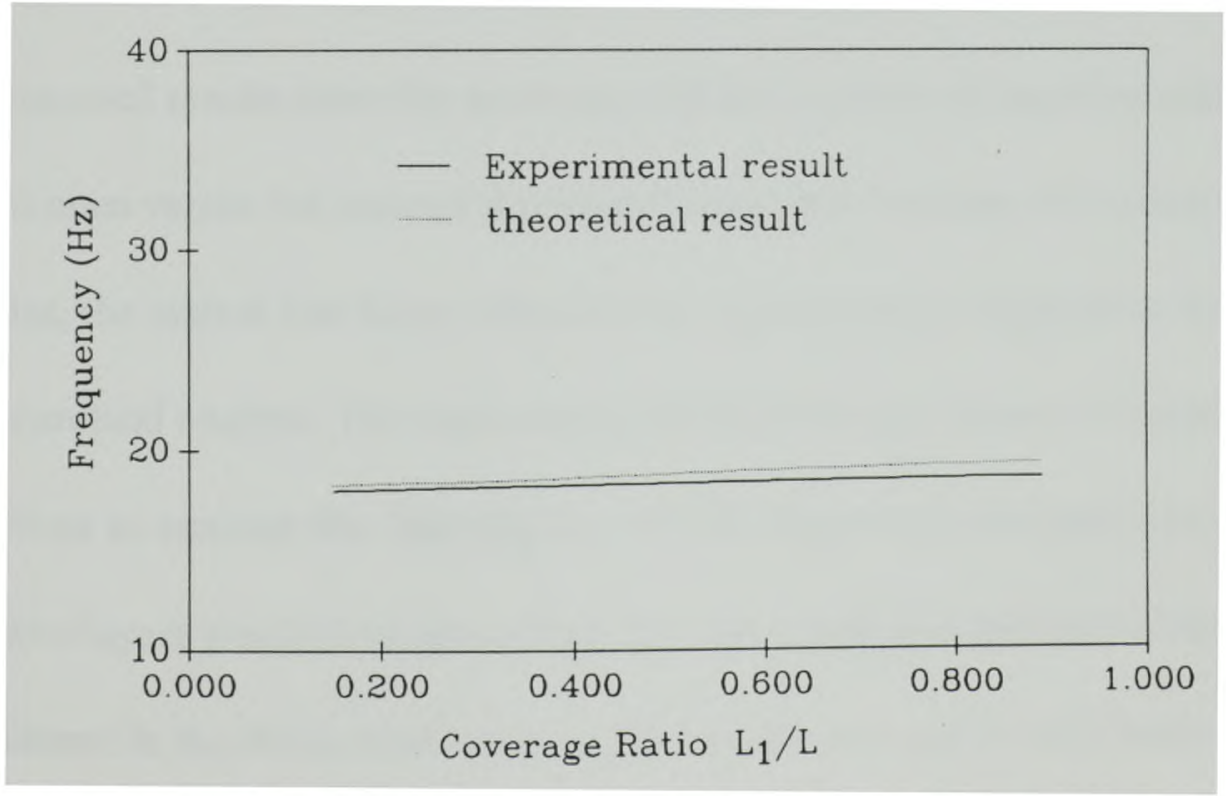

Figure 5.4 Coverage length ratio vs. natural frequency of the system 
the figure that the increase of the coverage length will increase the natural frequencies. This tendency confirms the numerical results obtained in Chapter 2 . In additions, the theoretical results give larger natural frequency compared to the experimental result. For small coverage, the difference in the natural frequency obtained by experiment and by numerical method is small; this different increases with coverage but the maximum error is less than $5 \%$.

\section{Case 2 End Mass Effects}

Figure 5.5 gives the comparison of system loss factor for different mass ratios (end mass to mass of the beam). The solid line represents the experimental result and the dotted line represents the theoretical result. Both the theoretical and experimental results show the tendency that the increase of the mass ratio (the ratio of end mass versus the mass of the whole beam) will decrease the system loss factor. Besides, the system loss factor obtained by experiments is larger than that obtained by theoretical analysis. The explanation is that in the experiments, we used the epoxy adhesives to connect the damping layers with beam and constraining layers. These adhesive layers would dissipate certain amount of energy while such effects were not considered in the theoretical analysis. The maximum error for the system loss factor between theory and experiment is about $11 \%$.

Figure 5.6 shows the results of first natural frequency versus the mass ratio. 




Figure 5.5 The ratio of end mass to mass of beam vs. system loss factor

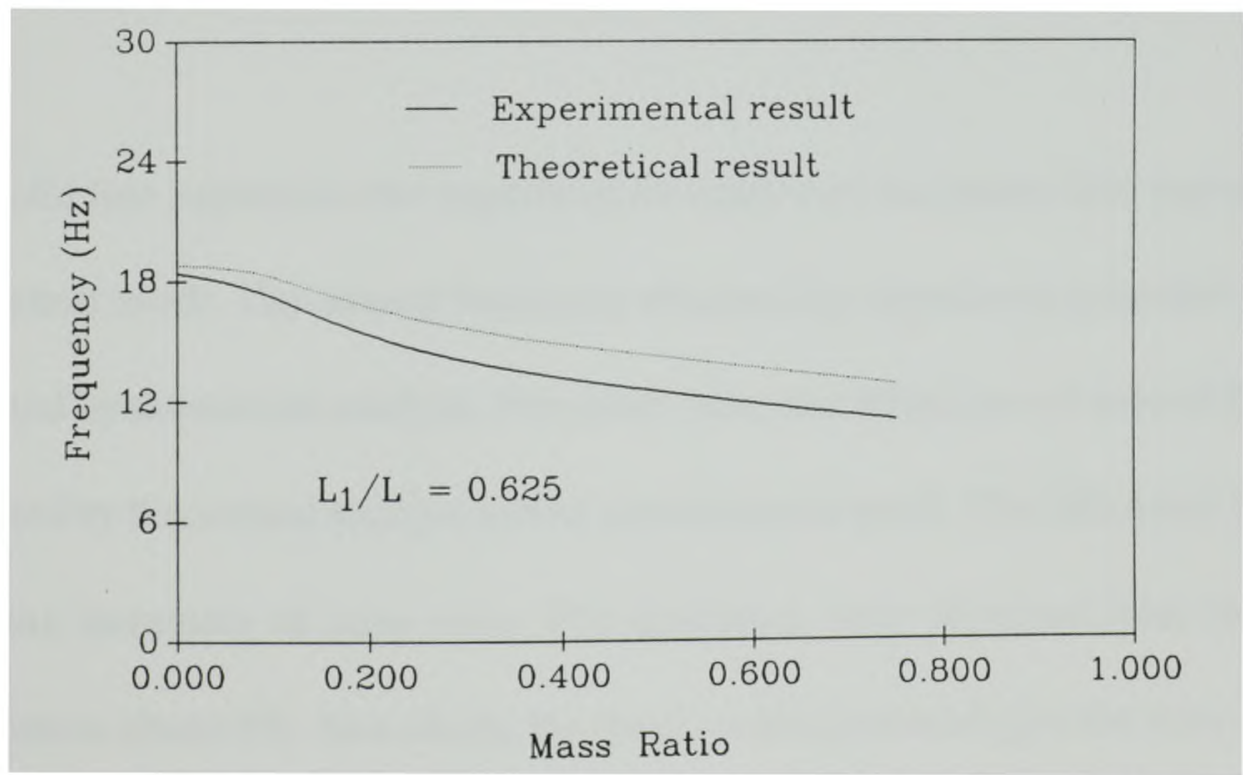

Figure 5.6 The ratio of end mass to mass of beam vs. natural frequency 


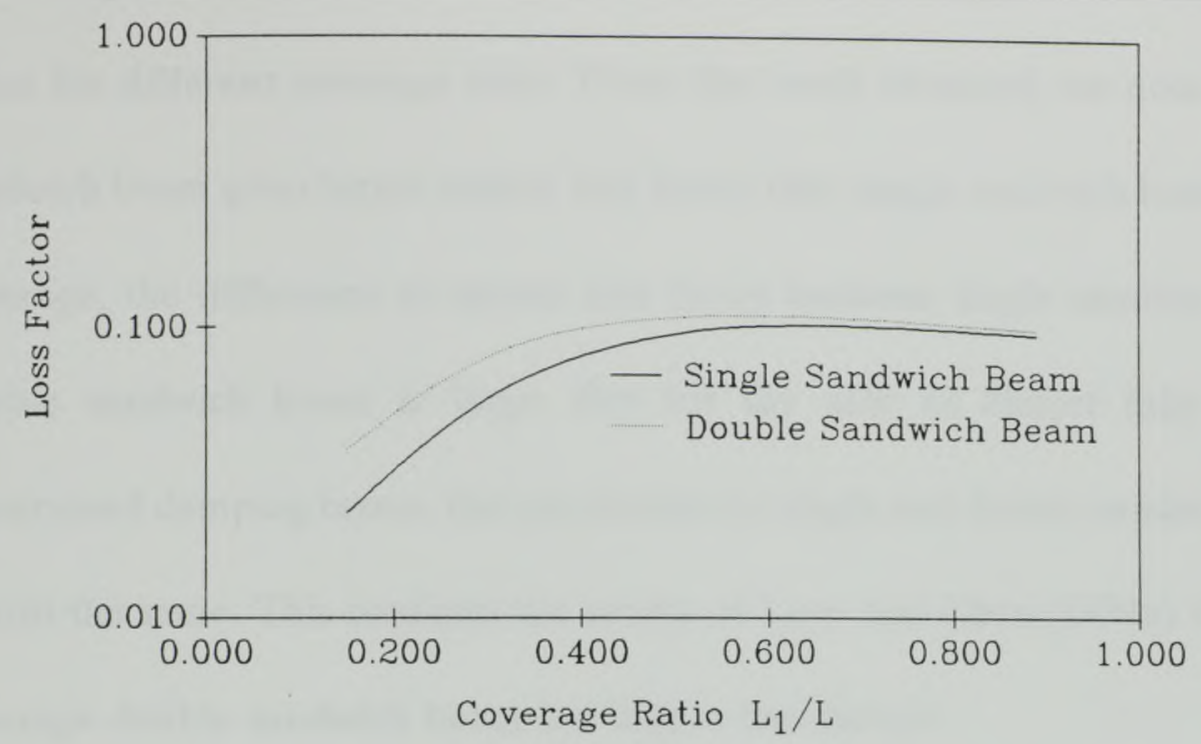

Figure 5.7 System loss factor comparison of single sandwich beam with double sandwich beam

The solid line represents the experimental result and the dotted line represents the theoretical result. The natural frequency obtained by experiment is smaller than that obtained by theoretical analysis. For small ratio, the difference of natural frequency obtained by theoretical analysis and by experiment is small. The difference increases with the increasing of mass ratio. The maximum error observed from theory and experiment about $9 \%$. As a whole, the theory and experiment give the same tendency that the increase of mass ratio will decrease the natural frequency. 


\section{Case 3 Single and Double Sandwich Beam}

Figure 5.7 compares the system loss factor of single and double sandwich beam for different coverage ratio. From the result obtained, we note that double sandwich beam gives larger system loss factor than single sandwich beam. For small coverage, the difference of system loss factor between single sandwich beam and double sandwich beam is large. But for the case of almost fully covered by constrained damping layers, the loss factors for single and double sandwich beam are almost the same. This confirms the results of Levy and Chen (1994a) that for small coverage double sandwich beam has higher loss factors. 
In this dissertation, the methods of active and/or passive vibration control for flexible structures were presented. The research covered four parts: 1) passive viscoelastic damping treatment; 2) active vibration control using layered SMA; 3) combined application of viscoelastic damping treatment and SMA; 4) experiments.

\section{1). Passive Damping Treatment}

In order to maximize damping and save weight of the structure and cost, a partially covered double sandwich cantilever beam model was presented. To take into account of end loads effect of elastic structures such as robot arm or manipulator, a model of partially covered double sandwich cantilever beam with mass at free end was discussed. Also a more accurate model (Timoshenko model) which may be used in the case of short beam and higher vibration modes was discussed. The effects of different parameters (material and geometrical parameters) on system frequency and loss factors were analyzed and discussed. From the numerical results, we conclude:

a) there exist optimal damping coverage length and optimal shear modulus of damping material which make the system loss factor maximum;

b) the addition of end mass will reduce the system natural frequency and the system loss factor; 
c) the frequency factor obtained by Euler beam model defined here is than that obtained by Timoshenko beam model;

d) in term of the system loss factor, the double sandwich beam is better than single sandwich beam for small coverage of constrained damping layer.

\section{2). Active Vibration Control}

Composites with SMA have the ability to change material properties, induce large internal forces in the materials, modify the stress and strain state of the structure. In order to use these properties, an active vibration control model by means of SMA actuators was discussed. The layered structure is easy to implement in real application, especially for existing structures. The temperature effect was also included in the analysis. From the numerical results, we know:

a) the change of physical properties in the SMA layer will change the system loss factor;

b) the change of SMA layer temperature will change the system loss factor; if the temperature is below the austenite starting temperature, or above the austenite finishing temperature, increase of temperature will decrease the system loss factor; if the temperature is between these temperature, increase of the temperature will increase the system loss factor; 
c) active vibration control is much better than no control case in terms of vibration amplitude and vibration response time;

d) the application of such model depends on the vibration control purpose. If it is used as an actuator, it could be used for low frequency vibration. If it is used for vibration tuning to avoid resonance, there is no restriction for the vibration frequency.

\section{3). Active and Passive Vibration Control Combination}

A model of combined application of SMA actuator and constrained viscoelastic damping treatment (called smart damping treatment) was presented. We can change the system frequency and loss factor by changing SMA properties. Vibration control results were discussed. For such a system, it is noted that:

a) in the case of active vibration control, vibration will die out much quicker than in the no control case with response amplitude having much smaller;

b) the introduction of damping treatment may improve the control results; c) although there is a limitation in the control frequency range because of the heating and cooling process of the SMA actuators, this model may also be used in high frequency applications because of the employment of the constrained viscoelastic layer. 


\section{4). Experiments}

Because of the limitation of experimental equipment, the only experiments performed were to determine the vibration characteristics of constrained viscoelastic damping structures with and without end masses. The experimental results were compared with theoretical results. Tendencies predicted by the theory were confirmed by the experiments. Quantitatively, good agreement was observed with the differences between theory and experiment having explained. The errors for system loss factor and natural frequency between theory and experiment are about $12 \%$ and $9 \%$, respectively.

5) Applications

The models studied here could be for vibration control in flexible robot structure, space manipulator system, suspension bridge, car, railway vehicle and many other structures.

\section{6). Future Research}

The following topic areas are to be investigated in the future:

a) Study of more compact system (Constraining layer of previous structure is replaced by SMA material which was suggested by Dr. Gareth J. Knowles);

b) study of partially covered SMA structure; 
c) Experimental verification of vibration control by means of SMA actuators or combined application of SMA actuator and constrained viscoelastic damping treatment. We will design a vibration signal sensing system, A/D and D/A converter system, control system (computer and software), and power supply system for SMA actuators;

d) application of theoretical and experimental results to more complex system, such as multibody systems that existed in real enviromental situations such as multi-link robot systems and large space manipulators. 


\section{BIBLIOGRAPHY}

Agbrasiere, J.A. and Groutenhuis, P. (1968) "Flexural Vibration of Symmetrical Multilayer Beams with Visco-elastic Layers", Journal of Mechanical Engineers, 10, pp269-281.

Agnes, G. S. and Napolitano, K. (1993) "Active Constrained Layer Viscoelastic Damping", ALAA/ASME Structures, Structural Dynamics and Materials Conference, pt. 6, pp3499-3503.

Alberts, T.E., Dikerson, S.L. and Book, W.J. (1986) "On the Transfer Function Modeling of Flexible Structures with Distributed Damping," in Robotics: Theory and Applications, F.W.Paul and K.Youcef-Toumi,Eds., ASME DSC-Vol.3.

Alberts, T.E., Love, L.J., Bayo E. and Moulin, H. (1990) " Experiments With End-Point Control of a Flexible Link Using The Inverse Dynamics Approach and Passive Damping," Proceedings of the 1990 American Control Conference, San Diego, CA, pp350-355.

Alberts, T.E., Xia, H. and Chen, Y. (1992) " Dynamic Analysis to Evaluate Viscoelastic Passive Damping Augmentation for the Space Shuttle Remote Manipulator System," Journal of Dynamic Systems, Measurement and Control, 114, pp468-475.

Amba-Rao, C. L. and Hussaini, M. Y. (1975) "On Free Vibration of Finite Beams with Partially Fixed End Conditions Carrying Masses", J. of Appl. Mech., 42(3), pp735-736.

Apollonov, V.V., Aksinin, V.I., Chetkin, S.A., Kijiko, V.V., Muraview, S.V. and Vdovin, G.V. (1992) "Magnetostrictive Actuators in Optical Design", Proceedings of SPIE- The International Society for Optical Engineering, 1543, pp313-324.

Arockiasamy, M., Neelakanta, P. S. and Sreenivasan, G. (1992) "Vibration Control of Beams with Embedded Smart Composite", J. Aerosp. Eng., 5(4), pp492498.

Asnani, N.T. and Nakra, B.C. (1970) "Vibration Analysis of Multilayered Beams Involving Visco-elastic Layer", Journal of Mechanical Engineering (India), 50, pp187-193. 
Bailey, T. and Hubbard,Jr., J.E. (1985) " Distributed Piezoelectric Polymer Active Vibration Control of a Cantilever Beam," Journal of Guidance, Control and Dynamics, 8(5), pp605-611.

Balas, M.J. (1978) " Active Control of Flexible Structures," Journal of Optimization Theory and Applications, 25(3), pp415-436.

Bar-kanal, I. and Kaufman, H. (1984) " Some Applications of Direct Adaptive Control to Large Structural Systems," Journal of Guidance, Control and Dynamics, 7(6), pp717-724.

Barker, D.K. (1989) "Active Dynamic Response Tuning of Adaptive Composite Utilizing Embedded Nitinol Actuators," M.S.Thesis, Department of Mechanical Engineering, Virginia Polytechnic Institute and State University.

Brinson, L.C. (1993) " One Dimensional Constitutive Behavior of Shape Memory Alloys: ThermomechanicalDerivation with Non-constantMaterial Functions and Redefined Martensite Internal Variable," Joumal of Intelligent Material Systems and Structures, 4, pp229-242.

Browning, D.R. and Medaugh, R.S. (1992) " Development of an Active Vibration Controller for an Elastic Structure," Proceedings of the 2nd International Congress on Recent Development in Air- and Structure-born Sound and Vibration, pp395-404.

Bryant, M.D., Fernandez, B., Wang, N., Murty, V.V., Vadlamani, V. and West, T.S. (1993) " Active vibration Control in Structures Using Magnetostrictive Terfenol with Feedback and/or Neural Network Controllers", Journal of Intelligent Material Systems and Structures, 4(4), pp484-489. 3174851

Buehler, W.J. and Wiley, R.C. (1965) "Nickel-Base Alloys," U.S.Patent

Chen, Q. and Levy, C. (1995a) "Active Vibration Control of Elastic Beam by Means of Shape Memory Alloy Layers", Proceedings of SPIE - The International Society of Optical Engineering, 2443, pp579-587.

Chen, Q. and Levy, C. (1995b) "Simplified Model for Combined Applications of Viscoelastic Material and Shape Memory Alloy to Vibration Control," Proceedings of SPIE - The International Society of Optical Engineering, 2443, pp481-490. 
Chen, Q. and Levy, C. (1994a) "Vibration Analysis of a Partially Covered Double Sandwich Cantilever Beam with Concentrated Mass at Free end," International Joumal of Solid and Structures, 31(7), pp2377-2391.

Chen, Q. and Levy, C. (1994b) "Smart Damping Treatment for Flexible Structure", Mat. Res. Soc. Symp. Proc., 360, pp527-532.

Chen, Q. and Xi., D. (1988a) "Transverse Displacement Reduction Effects of Damped Sandwich Beam Excited by Random Vibration from the Foundation", Acta Armamentarri (China), No.3, pp2-9.

Chen, Q. and Xi, D. (1988b) "Noise Reduction Effects of Damped Beam", Enviroment and Strength (China), No.4, pp24-29.

Chen, Q. and Xi, D. (1987) "An Improved Differential Equations for Damped Sandwich Beams," Journal of Zhejiang University, 21(3), pp32-38.

Chen, Q. (1986a) "Noise and Vibration Control of Damped Sandwich Beam", Master's Thesis, Zhejiang University,

P.R.China.

Chen, Q. (1986b) "Vibration and Noise Control Using Damped Structure", Pachaging World, 3, pp10-16.

Cheng, L. and Lapointe, R. (1994) "Shape Optimization of Viscoelastic Coating with Partial Coverage and Frequency-Temperature Effects", Proceedings of 3rd International Congress on Recent Developments in Air-and Structure-born Sound and Vibration, pp253-260.

Crawley, E.F. and Luis, J. (1987) " Use of Piezoelectric Actuator as Elements of Intelligent Structures," ALAA Journal, 25, pp1373-1385.

Ditaranto, R.A. (1965) "Theory of Vibratory Bending for Elastic and Viscoelastic Layered Finite Length Beams, " ASME Journal of Applied Mechanics, 87, pp881-886.

Duclos,T.G., Coulter J.P. and Miller, L.R. (1989) "Applications of Smart Materials in the Field of Vibration Control" in Smart Materiah, Structures, and Mathematical Issues pp1-9.

Edberg, D.L. and Bicos, A.S. (1991) "Design and Development of Passive Damping Concepts in Advanced Composite Large Space Structures", International SAMPE Symposium and Exhibition, 36, Covina, CA, USA, PP1-12. 
Fanson, J.L. and Garba, J.A. (1988) "Experimental Studies of Active Members in Control of Large Space Structures," ALAA paper 88-2207, in Proc. of ALAA |ASME/AHS 29th Structures, Structural Dynamics, and Materials Conference, pp9-17.

Flatau, A.B., Hall, D.L. and Schlesselman, J.M. (1993) " Magnetostrictive Vibration Control Systems", Journal of Intelligent Material Systems and Structures, 4(4), pp560-565.

Fujita, T., Shimotoku, K., Fujimoto, S., Katayama, H., Kobayashi, T. and Sakai, I. (1994) " Fundamental Study of Active Microvibration Control System Using Giant Magnetostrictive Actuator", Transactions of the Japan Society of Mechanical Engineers, Part C, 60, pp811-817.

Fukuda, T., Hosokai, H. and Kikuchi, I. (1990) "Distributed Type of Actuators by Shape Memory Alloy and Its Application to Underwater Mobile Robotic Mechanism", Proceedings of 1990 IEEE International Conference on Robotics and Automation, pp1361-1321.

Fukuda, T., Hosokai, H., Arai, F. and Kikuchi, I. (1990) "Underwater Propulsion Mechanism Using Distributed Type of Shape Memory Alloy Actuators", Proceedings of the 1990 ASME International Computers in Engineering Conference and Exposition, pp173-178.

Ganeriwala, S.N. (1994) "Characterization of Damping Properties of Nonlinear Viscoelastic Materials", Proceedings of 3rd International Congress on Recent Developments in Air- and Structure-born Sound and Vibration, pp395-402.

Garrison, M.R., Miles, R.N., Sun, J.Q. and Bao, W. (1992) "Effect of Partial Coverage on the Effectiveness of a Constrained Layer Damper on a plate", Proceedings of 2nd International Congress on Recent Developments in Air- and Structure-born Sound and Vibration, pp157-164.

Gaudreault, M.L.D., (1993) "Simultaneous Design of Active Vibration Control and Passive Viscous Damping," Journal of Guidance, Control and Dynamics, 16, pp654-661.

Gaudreault, M.L.D., Bagley, R.L. and Liebst, B.S. (1992) " Simultaneous Design of Active Vibration Control and Passive Viscoelastic Damping," Proceedings of the 2nd International Congress on Recent Developments in Air- and Structure-born Sound and Vibration, pp169-176. 
Graesser, E.J. and Cozzarelli, F.A. (1994) "A Proposed Three-Dimensional Constitu tive Model for Shape Memory Alloys," Journal of Intelligent Material Systems and Structures, 5, pp78-89.

Graesser, E.J. and Cozzarelli, F.A. (1991) "Shape Memory Alloys as New Materials for Aseismic Isolation," ASCE, Journal of Engineering Mechanics, 117(11), pp2590-2608,

Gurgoze, M. (1986) "On the Approximate Determination of the Fundamental Frequency of a Restrained Cantilever Beam Carrying a Tip Heavy Body", J. Sound \& Vib., 105, pp443-449.

Habib, M.S. (1991) " Active Parametric Damping of Distributed Parameter Beam Transverse Vibration," Joumal of Dynamic System, Measurement and Control, 113, pp295-299.

He, S. and Rao, M.D. (1992) "Damping of Laminated Composite Beams with Multiple Visco-elastic Layers", Proceedings of 2nd International Congress on Recent Developments in Air- and Structure-born Sound and Vibration, pp265-270.

Holnicki-Szulc, J. (1993) "Optimal Location of Actuators for Active Damping of Vibration," ALAA Journal, 31, pp1274-1279.

Inman, D.J. (1989) Vibration with control, measurement and stability, Prentice Hall, Englewood Cliffs, New Jersey.

Jones, D.I.G. (1974) "Temperature-Frequency Dependence of Dynamic Properties of Damping Materials", Journal of Sound and Vibration, 33(4), pp451-470.

Katsutoshi Kuribayashi (1993) "Trial Fabrication of Micro Sized Arm Using Reversible TiNi Alloy Thin Film Actuators", Proceedings of 1993 IEEE RSJ International Conference on Intelligent Robots and Systems, pp1697-1702.

Katsutoshi Kuribayashi (1991) "Improvement of the Response of an SMA Actuator Using a Temperature Sensor", Int. J. Rob. Res. , 10(1), pp13-20.

Kerwin, E.M. (1959) " Damping of flexural waves by a Constrained Visco-elastic layer," Journal of the acoustical Society of America, 31, pp952-962.

Knowles, G. (1990) "On Regulation Problems for Point Sensing and Control Systems," Proceedings of the 29th IEEE Conference on Decision and Control, Part 6, pp2957-2959. 
Koshigoe, S. (1993) " A Unified Analysis of Both Active and Passive Damping for a Plate With Piezoelectric Transducers," Journal of The Acoustical Society of America, 93, pp346-355.

Lagoudas, D. C. Boyd, J. G. and Bo, Bo. (1992) "Analysis of a Coupling Made of Shape Memory Alloy and Its Dynamic Response due Impacts," Joumal of Vibration and Acoustics, 114, pp297-304.

Lall, A.K., Asnani, N.T. and Nakra, B.C. (1988) "Damping Analysis of Partially Covered Sandwich Beams", Journal of Sound and Vibration, 123, pp247-259.

Lall, A.K., Asnani, N.T. and Nakra, B.C. (1987) "Vibration and Damping Analysis of a Rectangular Plate with Partially Covered Constrained Viscoelastic Layer", Transactions ASME, Journal of Vibration, Acoustics, Stress and Reliability in Design, 109, pp241-247.

Lashlee, R.W., Damle, R, Rao V.S. and Kern, F.J. (1993) "Identification and Robust Control of Flexible Structures Using Shape Memory Actuators", Proceedings of SPIE - The International Society for Optical Engineering, 1919, pp271-282.

Laura, P. A. A., Susemihl, E. A., Pombo, J. L., Liusoni, L. E. and Gelos, R. (1977) "On the Dynamic Behavior of Structural Elements Carrying Elastically Mounted, Concentrated Masses", Appl. Acoustics, 10, pp121-145.

Laura, P. A. A., Filipich, C. P. and Cortinez, V. H. (1987) "Vibrations of Beams and Plates Carrying Concentrated Masses", J. Sound \& Vib., 117, pp459-465.

Levraea,Jr., V. and Rogers, L. (1992) "Passive Damping Applied to Alrcraft Wing Skin," Proceedings of 2nd International Congress on Recent Developments in Airand Structure-born Sound and Vibration, pp287-294.

Levy, C. and Chen, Q. (1994a) "Vibration Analysis of the Partially Covered Double Sandwich-type Cantilever Beam," Joumal of Sound and Vibrations, 177(1), pp15-29.

Levy, C. and Chen, Q. (1994b) "Vibration Characteristics of Partially Covered Double Sandwich Cantilever Timoshenko Beam", Proceedings of 3rd International Congress on Recent Developments in Air- and Structure-born Sound and Vibration, pp433-440.

Liang, C. and Rogers, C.A. (1993) " Design of Shape Memory Alloy Springs with Applications in Vibration Control," Journal of vibrations and Acoustics, 115, pp129-135. 
Lukasiewich, S. and Xia, Z.Q. (1993) "Nonlinear, Damped Vibrations of Sandwich Plates with Time-dependent Temperature", ASME, Design Engineering Division (Publication) De-Vol.56, pp427-436.

Magrab, E. B. (1979) Vibrations of Elastic Structural Members, Sijhoff and Noordhoff International Publications B. V., Alphen aan den Rijn, The Netherlands.

Mantena, P.R. (1991) "Optimal Constrained Viscoelastic Tape Lengths for Maximizing Damping in Laminated Composites", ALAA Journal, 29, pp1678-1685.

Matsuno, F. and Sakawa, Y. (1990) "A Simple Model of Flexible Manipulator with Six Axes and Vibration Control by Using Accelerometers," Journal of Robotic Systems, 7(4), pp575-597.

Mead, D.J. (1982) "A Comparison of Some Equations for the Flexural Vibration of Damped Sandwich Beams," Journal of Sound and Vibrations, 83(3), pp362-377.

Mead, D.J. and Markus, S. (1969) "The Forced Vibration of a Three-Layer, Damped Sandwich Beam with Arbitrary Boundary Conditions," Journal of Sound and Vibration, 10(2), pp163-175.

Nagaya, K. and Hirata, Y. (1994) "Micromechanics of Active Composite with SMA Fibers," Journal of Engineering Materials and Technology, 116, pp337-347.

Nagaya, K., Takeda, S., Tsukui, Y. and Knmaido, Y. (1987) " Active Control Method for Passing through Critical Speeds of Rotating Shafts by Changing Stiffness of the Supports with Use of Memory Metals," Journal of Sound and Vibration, 111(2), pp307-315,

Nashif, A. (1992) " Recent Application of the Passive Damping Technology," Proceedings of the 2nd International Congress on Recent Developments in Air-and Structure-born Sound and Vibration, pp189-195.

Newsom, J.R., Layman, W.E., Waites H.B. and Hayduck, R.J. (1990) " The NASA Controls-Structures Interaction Program," 41st Congress of the International Astronautical Federation, Dresden, GDR, IAF-90-290.

Pan, H. H. (1965) "Transverse Vibration of an Euler Beam Carrying a System of Heavy Bodies", J. of Appl. Mech., 32(2), pp434-437. 
Plunkett, R.and Lee, C.T. (1970) "Length Optimization for Constrained Viscoelastic Layer Damping," Journal of Acoustical Society of America, 48(1), pp150-161.

Pruce, J.C., Kokkinos, F. and Syyrakos, C.C. (1989) " Advanced Joining Concepts for Passive Vibration Control," Journal of Aerospace Engineering, 1(4), pp193-205.

Pu, Z.J. (1995), "Private Conversation".

$\mathrm{Pu}$, Z.J. and Wu, K.H. "The Martensite Transformation and Shape Memory effect of NiTi-Hf Alloys," Journal of Materials and Engineering, Accepted.

Rami Reddy, K. and Amba-Rao, C. L. (1973) "Vibrations of Beams Carrying Masses-Transfer Matrices Approach", J. of the Aero. Soc. of India, 25(3), pp107-110.

Rao, D. K. (1977) "Vibrations of Short Sandwich Beams. J. Sound \& Vib., 52(2), pp253-263.

Rao, D.K. (1978) "Frequency and Loss Factors of Sandwich Beams Under Various Boundary Conditions," Journal of Mechanical Engineering Science, 20(5), pp271-282.

Rao, S. S. (1990) Mechanical Vibrations, 2nd Edition, Addison-Wesley Publishers, New York, Chapter 8.

Reinhorn, A.M. and Manolis, G.D. (1985) " Current State of Knowledge on Structural Control", Sound Vibr. Dig. 17, pp7-16.

Rogers C.A., Liang, C. and Barker, D.K. (1989) "Dynamic Control Concepts Using Shape Memory Alloy Reinforced Plates," in Smart Material, Structures and Mathematical Issues(Technomic, Lancaster,PA), pp39-62.

Rogers, C.A. (1990) "Active Vibration and Structural Acoustic Control of Shape Memory Alloy Hybrid Composites:Experimental Results," Journal of the Acoustical Society of America, 88(6), pp2803-2811.

Rogers, C.A., Liang, C. and Fuller, C.R. (1991) " Modeling of Shape Memory Alloy Hybrid Composites for Structural Acoustic Control," Journal of the Acoustical Society of America, 89(1), pp210-220.

Sakawa, Y., Matsuno, F. and Fukushima, S. (1985) "Modeling and Feedback Control of a Flexible Arm," Journal of Robotic Systems, 2(4), pp453-472. 
Saunders, W.R., Robertshaw, H.H. and Rogers, C.A. (1990) " Experimental Studies of Structural Acoustic Control for a Shape Memory Alloy Composite Beam," in Proceedings of the 31st Structures, Structural Dynamics and Materials Conference, ALAA 90-1090.

Scott, M.A. (1993) "Active Vibration Damping of the Space Shuttle Remote Manipulator System," Journal of Guidance, Control and Dynamics, 16, pp275-280.

Scott, M.A., Gilbert, M.G. and Demeo, M.E. (1993) " Active Vibration Damping of the Space Shuttle Remote Manipulator System", Journal of Guidance, Control and Dynamics, 16(2), pp275-280.

Simonian, S.S., Major, C.S. and Gluck, R., (1984) "An Experimental Study of Passive Damping and Active Control of Large Space Structures," AFWAL Vibration Damping 1984 Workshop Proceedings, (AD-A152547).

Stokey, W. F. and Zorowski, C. F. (1959) "Normal Vibrations of a Uniform Plate Carrying Any Number of Finite Masses", J. Appl. Mech., 81, pp210-216.

Tzou, H.S. (1988) "Control of Flexible Structure Vibration by Distributed Viscoelastic Damping," in Machine Dynamics and Engineering Applications, Xian Jiaotong University Press, China, pp15-19.

Tzou, H.S. (1989) "Integrated Distributed Sensing and Active Vibration Suppression of Flexible Manipulators Using Distributed Piezoelectrics," Journal of Robotic Systems, 6(6), pp745-767.

Tzou, H.S. and Anderson, G.L. (1992) Intelligent Structural System, Kluwer Academic Publishers.

Tzou, H.S. and Wan, G.C. (1990) " Distributed Structural Dynamics Control of Flexible Manipulators-I. Structural Dynamics and Distributed Viscoelastic Actuator", Computers \& Structures, 35(6), pp669-677.

Weaver,Jr., W., Timoshenko, S.P. and Young, D.H. (1990) Vibration Problem in Engineering, John Wiley \& Sons, Inc..

Wojciech, S., Klosowicz, M. and Nadolski, W. (1990) "Nonlinear Vibration of a Simply Supported, Viscoelastic Inextensible Beam and Comparison of Four Methods", Acta Mech. 85, pp43-54.

Wu, K.H. and Liu, C.T., (1993) "The Effect of Compressive Stress on Reaction Sintering of $\mathrm{Ni}_{3} \mathrm{Al}$," Metallurgical Transction, (in press). 
Wu, K.H., Liu, Y.Q., Maich, M. and Tseng, H.K. (1994) "The Mechanical Properties of a NiTi-Pd High Temperature Shape Memory Alloy," Proceedings of the SPIE - The International Society of Optical Engineering, 2189, pp306-313.

Xi, D., Chen, Q. and Cai, G. (1986) "Control Effects of Damped Sandwich Beam on Random Vibration," ASME Joumal of Vibration, Acoustics, Stress and Reliability in Design, 108, pp65-68.

Zhou, H.S. (1991) "Distributed Modal Identification and Vibration Control of Continua: Theory and Applications", Trans ASME Journal of Dynamic System, Measurement and Control, 113, pp494-499. 


\section{VITA}

October 2, $1961 \quad$ Born, Zhejiang, China

$1978-1982$

Shanghai Institute of Railway Technology, China

Bachelor of Science in Mechanical Engineering

1982-1983

Tsing Tao Locomotive and Railway Vehicle Factory

Ministry of Railway, China

Assistant Engineer

1983-1996

Zhejiang University Hangzhou, China

Master of Science in Mechanics Department

1985

First prize winner of Zhejiang University

1986-1991

Zhejiang University, HangZhou, China

Mechanics Department

Lecturer

1991-1993

Florida International University, Miami,FL

Mechanical Engineering Department

Research Associate

1993-present: $\quad$ Florida International University, Miami, FL

PhD in Mechanical Engineering

1995

Second prize winner of graduate students scholarly forum, FIU;

\section{PUBLICATIONS}

In Journals and Magazines

1. C.Levy and Q.Chen,(1994) "Vibration Analysis of a Partially Covered, Double Sandwich-Type, Cantilever Beam", Journal of Sound and Vibration, 177(1), pp. 15-29.

2. Q.Chen and C.Levy,(1994) "Vibration Analysis of a Partially Covered, Double Sandwich Cantilever Beam with Concentrated Mass at Free End", International Journal of Solids and Structures, 31(17), pp.2377-2391. 
3. Q.Chen and D.Xi,(1989), "Nonlinear and Parametric Vibration of an Elastic Oscillator with Dynamic Coils", Journal of Zhejiang University, 26(5).

4. Q.Chen, (1989), "The Principles for Puncher Improvement", Packaging World, vol.13.

5. Q.Chen, (1989), "Failure Analysis of Packaging Box for Boiled Bamboo Shoot", Packaging World, vol.15.

6. Q.Chen and D.Xi,(1988) "Effects of Vibration Reduction of Transverse Displacement on Damped Sandwich Beam Excited by Random Vibration from the Foundation", Acta Armamentarri, No.3, pp2-9.

7. Q.Chen and D.Xi, (1988), "Vibration Isolation and Cushioning Experiment for Packaging System', Packaging World, Vol.6.

8. Q. Chen and D. Xi, (1988), "Noise Reduction Effects of Damped Beam", Environment and Strength, (China), No.4, pp24-29.

9. M.Wu, G.Luo and Q.Chen, (1988), "More about Packaging Mechanics of Commodities in Transportation", Zhejiang Transportation, (China), Vol.24.

10. Q.Chen and D.Xi, (1987), "An Improved Differential Equation of Damped Sandwich Beam", Journal of Zhejiang University, (China), 21(3), pp32-38.

11. D. Xi, Q. Chen and G. Cai, (1986), "Control Effects of Damped Sandwich Beam on Random Vibration", ASME,Journal of Vibration, Acoustics Stress and Reliability in Design", Vol.108, pp. 65-68.

12. D. Xi and Q. Chen, (1986), "Definition, Classification and Function of Packaging", Packaging World, (China), Vol.1.

13. Q. Chen, (1986), "Vibration and Noise Control Using Damped Structure", Packaging World, Vol.3.

14. Q.Chen, (1984), "An Investigation of Some Playing Techniques of Chinese Musical Instrument-Pipa", Journal of Shanghai Music Conservatory".

\section{Conference Proceedings}

1 Q.Chen and C.Levy, (1995) "Simplified Model for Combined Applications of Viscoelastic Material and Shape Memory Alloy to Vibration Control" 
Proceedings of SPIE - The International Society of Optical Engineering, 2443, pp.579-587.

2 Q.Chen and C.Levy, (1995) "Active Vibration Control of Elastic Beam by Means of shape Memory Alloy layers", Proceedings of SPIE - The International Society of Optical Engineering, 2443, pp. 481-490

3 C.Levy and Q.Chen, (1994) "Vibration Characteristics of Partially Covered Double Sandwich Cantilever Timoshenko Beam", Proceedings of the 3rd International Congress on Recent Development in Air- and Structure-Borne Sound and Vibration, Canada,1994.

4 Q.Chen and C.Levy, (1994) "Smart Damping Treatment for Flexible Structure", Proceedings of MRS 94 Conference, 360, pp.527-532.

5 C.Levy and Q.Chen, (1993) "Vibration Analysis of a Partially Covered Double Sandwich Cantilever Beam with Concentrated Mass at Free End", Proceedings of 4th Biennial ASME Conference on Mechanical Vibration and Noise, Albuquerque, USA.

6 C.Levy and Q.Chen, (1993) "Improved Differential Equations of a Partially Covered,Double Sandwich Type Cantilever Beam", Abstract Proceedings of Joint ASCE-ASME-SES Meeting, Virginia, USA.

7 Q.Chen and C.Levy, (1992) "A Simple Method of Structural Parameter Modification for a MDOF System", Proceedings of the 2nd International Congress on Recent Development in Air- and Structure-Borne Sound and Vibration, Alabama, USA.

8 M.Wu, G.Luo and Q.Chen, (1988) "More about Packaging Mechanics of Commodities in Transportation", Proceedings of the 2nd National Conference on Packaging Dynamics and Design. (Hangzhou, China).

9 D.Xi and Q.Chen, (1997) "The Vibration and Shock Testing in China, Pt.1", Proceedings of 5th IAPPI (England).

10 D.Xi and Q.Chen, (1987) "The Vibration and Shock Testing in China, Pt.2", Proceedings of 5th IAPPI (England).

11 D.Xi and Q.Chen, (1986) "Response of Packaged Articles Connected By Elastic Element to Random Excitation", Proceedings of the 1st National Conference on Packaging Dynamics and Design, (Hangzhou, China). 
12 D.Xi and Q.Chen, (1986) "Noise Reduction Effects of Sandwich Beam", Proceedings of 12th ICA, Canada.

13 Q.Chen and X.Sun, (1986) "Study on Chinese Musical Instrument-Pipa", Proceedings of 12th ICA, Canada.

14 D.Xi and Q.Chen, (1985) "Response of a Nonlinear System under parametric and Forcing Excitation", Proceedings of International Conference on Nonlinear Mechanics (Shanghai,China).

15 D.Xi and Q.Chen, (1985) " Displacement Control Effects of Sandwich Beam to Random Base Excitation", Presented at The 3rd East China Conference on Solid Mechanics, HangZhou, China.

\section{Papers Submitted}

1 Q.Chen and C.Levy, "Vibration Characteristics of Cantilever Beam with Viscoelastic Layer and Shape Memory Alloy Layer" Submitted to Journal of Dynamic Systems, Measurement, and Control. (Dec. 1994).

2 Q.Chen and C.Levy, "Vibration Characteristics of Partially Covered Double Sandwich Cantilever Timoshenko Beam", Submitted to International Journal of Solids and Structures. (Dec, 1994).

3 Q.Chen and C.Levy, "Active Vibration Control of Elastic Beam by means of Shape Memory Alloy layers", Submitted to Journal of Sound and Vibration (April, 1995).

\section{BOOK AND TEACHING MATERIALS}

1 Q.Chen," Vibration, Impact and Noise Control", 1985, for graduate students in Mechanics Department of Zhejiang University, China.

2 D.Xi, Q.Chen and Z.Wang, "Design of Packaging Structures", Zhejiang University Press, March,1994. 